\title{
Exploring natural variation in sucrose metabolism to improve tomato growth and development at sub-optimal temperature
}





\section{Exploring natural variation in sucrose}

metabolism to improve tomato growth and development at sub-optimal temperature

Quy Dung Dinh 


\section{Thesis committee}

\section{Promotors}

Prof. Dr Luisa. M. Trindade

Personal chair, Bioresources Breeding and Genetics

Wageningen University \& Research

Prof. Dr Richard. G. F. Visser

Professor of Plant Breeding

Wageningen University \& Research

\section{Other members}

Prof. Dr Mark G. M. Aarts, Wageningen University \& Research

Prof. Dr Christa S. Testerink, Wageningen University \& Research

Prof. Dr Jan van den Berg, University of Maastricht

Dr Rene M. Klein Lankhorst, Wageningen University \& Research

This research was conducted under the auspices of the Graduate School of Experimental Plant Sciences (EPS). 


\title{
Exploring natural variation in sucrose metabolism to improve tomato growth and development at sub-optimal temperature
}

\author{
Quy Dung Dinh
}

Thesis

submitted in fulfilment of the requirements for the degree of doctor at Wageningen University by the authority of the Rector Magnificus, Prof. Dr A.P.J. Mol, in the presence of the Thesis Committee appointed by the Academic Board to be defended in public on Wednesday 9 September 2020 at 11:00 a.m. in the Aula. 
Quy Dung Dinh

Exploring natural variation in sucrose metabolism to improve tomato growth and development at sub-optimal temperature, 146 pages.

$\mathrm{PhD}$ thesis, Wageningen University, Wageningen, the Netherlands (2020) With references, with summary in English

ISBN 978-94-6395-421-1

DOI https://doi.org/10.18174/523153 


\section{Table of contents}

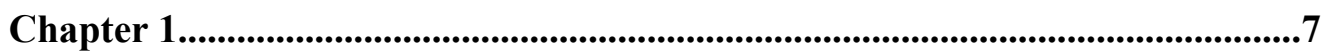

General Introduction

Chapter 2

High-Altitude Wild Species Solanum arcanum LA385 - A Potential Source for Improvement of Plant Growth and Photosynthetic Performance at Suboptimal Temperatures

Chapter 3..

Exploring natural genetic variation in tomato sucrose synthases on the basis of increased kinetic properties

Chapter 4.

Introgression of a region harboring a sucrose synthase allele from a wild relative into cultivated tomato increases dry matter allocation to fruits

Chapter 5.

General Discussion

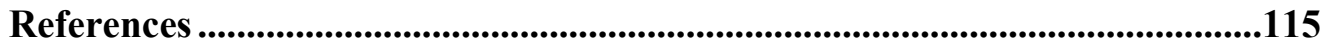

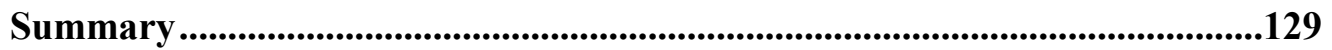

Acknowledgments ...........................................................................................................133

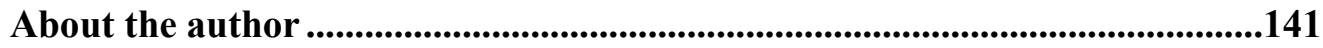

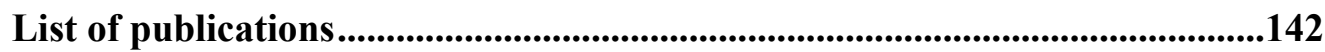

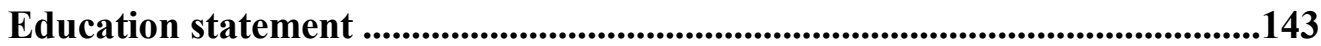





\section{Chapter 1}

\section{General Introduction}

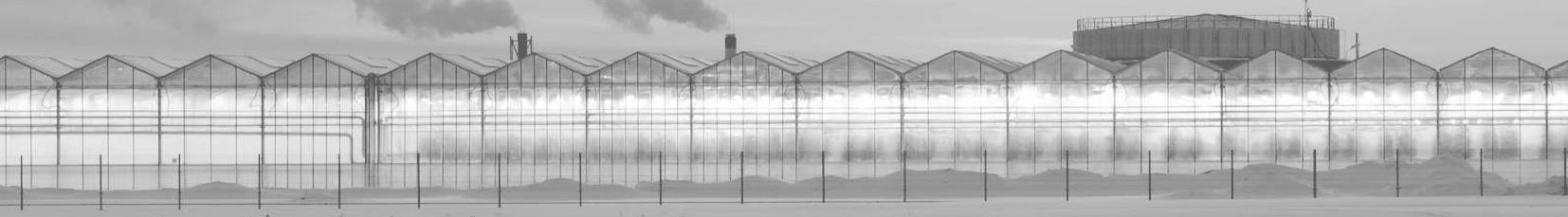

w a w

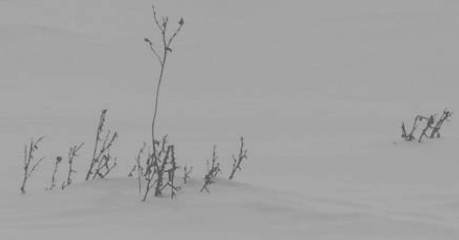




\section{Resilient crops for a sustainable economy}

As the world population is predicted to reach nearly 10 billion in 2050 (United Nations 2017), it is important to meet the increasing food demand. From an environmental point of view we are facing new challenges caused by the effects of climate change, such as more severe, prolonged, and frequent heat waves, cold spells, droughts, storms, etc. (National Climate Assessment 2014). In order to secure the food production in such challenging conditions, adapted or new crop varieties and alternative production systems are needed. On the one hand it would comprise technical advance such as growing crops in climate controlled farms with more advanced and innovative technologies, e.g. in a net energy producer glasshouse, or in a shipping container like a system called Grow Pod Solutions (www.growpodsolutions.com) (Fig. 1). On the other hand, advances could be achieved by breeding programs in which new varieties are bred with specific traits, such as heat, drought or cold tolerance. A combination of both agronomic solutions and novel varieties will enable to meet the challenges in food production. This thesis focuses on breeding related aspects which can play an important role in more sustainable crop cultivation for food production. In the following sections, I will first review the current status of greenhouse horticulture in the Netherlands in relation to efficient and sustainable cultivation. Subsequently I will introduce several aspects that are essential for plant growth, development and yield.
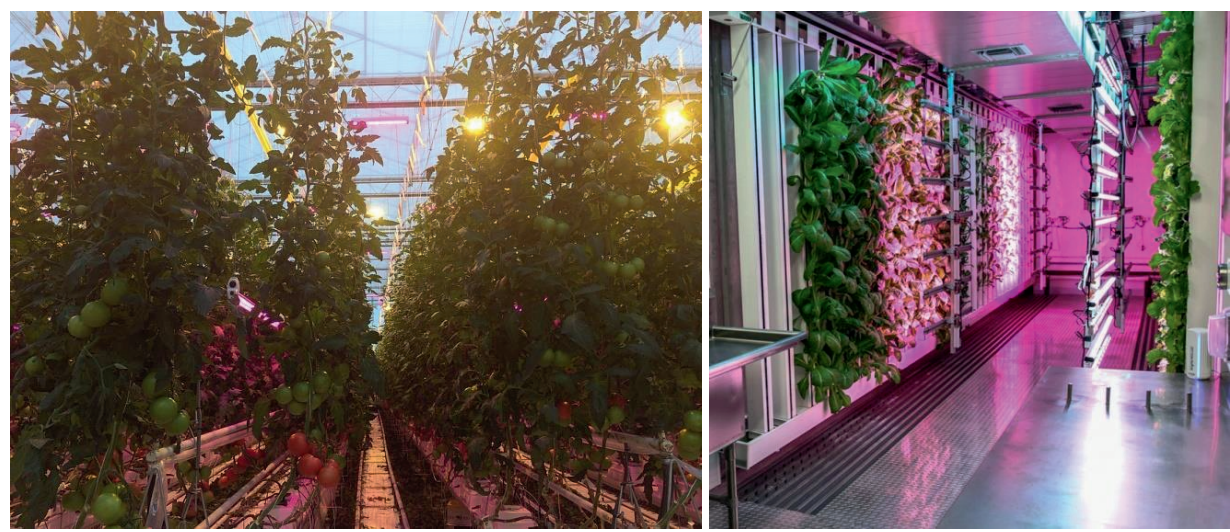

Figure 1. Examples of crops cultivation in climate controlled farms. The left panel shows a cultivation of tomatoes in a glasshouse, while the right panel shows a cultivation of leafy crops in a shipping container from a system called Grow Pod Solutions (sources: www.agrocontrol.nl and www.growpodsolutions.com). 


\section{Dutch greenhouse horticulture}

The Netherlands is a small country with a land area of $41,526 \mathrm{~km}^{2}$ but it is a leader in exporting agriculture products. In 2018, its export of agricultural goods was ranked second place globally, just after the United States of America with land area of 9.6 million $\mathrm{km}^{2}$ (MONGABAY 2008, Dillinger 2017). The total agricultural exports from the Netherlands in 2018 was worth 90.3 billion euros, in which export of vegetables was among the top five export products and contributed 6.6 billion euros (Dolman et al. 2019). The top five vegetables grown in the greenhouse with the largest cropping area and yield in 2018 were tomato 1,788 ha and 910 million $\mathrm{kg}$ (equivalent of $50.9 \mathrm{~kg} / \mathrm{m}^{2}$ ), cucumber 563 ha and 410 million $\mathrm{kg}\left(72.9 \mathrm{~kg} / \mathrm{m}^{2}\right)$, pepper 1,311 ha and 355 million $\mathrm{kg}\left(27.1 \mathrm{~kg} / \mathrm{m}^{2}\right)$, courgette $473 \mathrm{ha}$ and 19.8 million $\mathrm{kg}\left(4.2 \mathrm{~kg} / \mathrm{m}^{2}\right)$, and eggplant 105 ha and 55 million $\mathrm{kg}\left(36.6 \mathrm{~kg} / \mathrm{m}^{2}\right)(\mathrm{CBS}$ 2019). These yields from the Dutch greenhouse cultivation are known to be much higher compared to other countries. For example, according to a market report the Dutch tomato growers achieved an average of $50.7 \mathrm{~kg} / \mathrm{m}^{2}$ compared to $9.03,8.62$, and $8.08 \mathrm{~kg} / \mathrm{m} 2$ in US, Spain, and Morocco, respectively in 2016 (HortiDaily 2018). However, to achieve these high yields in greenhouse horticulture under the Dutch temperate climate, it requires very high energy consumption for the cultivation. The energy costs correspond to about $20-30 \%$ of the total production costs and correspond to energy used for heating, lighting and supplying $\mathrm{CO}_{2}$ in the greenhouse (Hemming 2010). As a result, food production using greenhouse horticulture in The Netherlands is deemed to have a high impact on the environment, which needs to be improved by decreasing it. As a result, a recent environmental law in the Netherlands has been proposed to address the impact of $\mathrm{CO}_{2}$ emission from different sectors including greenhouse horticulture, and to achieve these two goals: i. having fully sustainable and economically viable energy supply by 2050 , and ii. reducing $49 \%$ and $95 \%$ lower in $\mathrm{CO}_{2}$ emission in 2030 and 2050 than in 1990, respectively (Goverment of the Netherlands 2016, Groen Links 2018). In the last decade, the Dutch greenhouse horticulture has been making substantial progress to alleviate the negative impact on the environment by reducing $\mathrm{CO}_{2}$ emission, in which the $\mathrm{CO}_{2}$ emission level in 2016 was $18 \%$ below the level of 1990 (WUR and Kas als Energiebron 2016). The main factor for this reduction was the energy savings by the innovative development of intensification and the decreased extensification. Some of the examples in the innovative development of intensification in greenhouse horticulture are: covering the greenhouse with better screens to conserve heat, using diffuse glass to have more even sunlight distribution, using more energy efficient lighting supplement such as 
LED, etc. One area that could be further explored, in the context of further reducing energy consumption, is a better understanding of photosynthesis efficiency, growth and plant development under sub-optimal temperatures in greenhouses. To address the important role of photosynthesis in bioenergy and food production, Wageningen University \& Research coordinated a five-year research program called Toward BioSolar Cells. The focus of this program was mostly on three topics: 1) Systems biology of photosynthetic processes, 2) Reengineering organisms for optimal photosynthetic energy conversion into biomass, and 3) The design of systems for energy-tapping before the energy is converted into biomass (Wageningen UR 2008). The research within the consortium involved the study of model plants as Arabidopsis and several crops with great relevance for The Netherlands, tomato, potato, and sugar beet. This thesis is embedded in the second theme, where different disciplines such as modelling, biophysics, plant physiology, molecular biology, and genetics come together to address this complex research question.

\section{Photosynthesis}

Photosynthesis is at the basis of biomass production of autotrophic organisms, such as plants, some bacteria and protists, and indirectly of all life on earth (Hohmann-Marriott and Blankenship 2011). This basic process enables fixation of atmospheric $\mathrm{CO}_{2}$ into triose phosphate, from which all other plant components can be synthesized. In plants, photosynthesis occurs to a large extent in chloroplast-containing mesophyll cells (MC) of source leaves which are defined as photoassmilate net-producers. Photosynthesis has two stages. The first stage is called light dependent process (also known as Light Reactions), in which via several reaction steps the sunlight energy is converted into energy carrier molecules adenosine triphosphate (ATP) and nicotinamide adenide dinucleotide phosphate (NADPH). In addition, water is split and oxygen is released into the atmosphere as the byproduct. The second stage is called light independent process (also known as Dark Reactions). In this second stage, the reductive pentose phosphate cycle (Calvin-Benson cycle) uses the energy molecules ATP and NADPH, produced in the first stage, to fixates atmospheric carbon dioxide $\left(\mathrm{CO}_{2}\right)$ and produces triose phosphate (triose-P) as the end fixate (Fig. 2). The produced triose-P has three destinies concerning carbon partitioning: i. it stays in the Calvin-Benson cycle to continue another $\mathrm{CO}_{2}$ assimilation reaction, ii. it can enter the 
synthesis of starch within the chloroplast, or iii. it can be exported into the cytoplasm for the synthesis of sucrose (Bornke and Sonnewald 2011).

\section{Sucrose biosynthesis in source leaves}

Triose-P formed via Calvin-Benson cycle can be used to synthesize sucrose in source leaves (Fig. 2). Triose-P in chloroplasts is translocated into the cytosol by triose-P/phosphate translocator (TPT), located in the membrane of the chloroplast envelop, in a strict exchange for inorganic phosphate molecule $\left(\mathrm{P}_{\mathrm{i}}\right)$ (Flügge and Heldt 1984). Subsequently triose-P is converted to fructose-1,6-biphosphate (FBP) by fructose-1,6-biphosphate aldolase, and then FBP to fructose-6-phosphate (F6P) by cytosolic fructose-1,6-biphosphatase. The next two phosphorylated sugars glucose-6-phosphate (G6P) and glucose-1-phosphate (G1P) are maintained in equilibrium with F6P by phosphoglucoisomerase and phosphoglucomutase, respectively. In the following step, uridine diphosphate glucose (UDP-Glc) and pyrophosphate $\left(\mathrm{PP}_{\mathrm{i}}\right)$ are generated by the catalysis of UDP-glc pyrophosphorylase on substrate G1P and uridine-5'-triphosphate (UTP). UDP-Glc in combination with F6P is then converted into sucrose-6-phosphate (S6P) by sucrose-6-phophate synthase. Finally, sucrose (Suc) and $\mathrm{P}_{\mathrm{i}}$ are formed by the irreversible reaction of sucrose phosphatase on substrate S6P. Sucrose biosynthesis also occurs during the night via the breakdown of starch in chloroplast. Starch breakdown by several enzymes, e.g. amylases, phosphorylases, debranching enzymes, disproportionating enzyme, leads to the formation of maltose or occasionally glucose, which is then transported from chloroplast to cytosol for further conversion to G6P by hexokinase (Smith et al. 2005, Xu et al. 2014, Smirnova et al. 2015). Subsequent reaction steps are similar as the sucrose biosynthesis during day time described above. Parallel with the sucrose synthesis in source leaves, trehalose, a recently discovered signal metabolite in plants, may be also produced in a small amount in cytoplasm. Trehalose-6-phosphate (T6P) synthase (TPS) synthesizes T6P and UDP by transferring Glc from UDP-Glc to G6P. Subsequently T6P is converted to trehalose by T6P phosphatase (TPP) (De Smet et al. 2000, Elbein et al. 2003). Trehalose may be eventually hydrolyzed by trehalase (TL) into two molecules of glucose. 


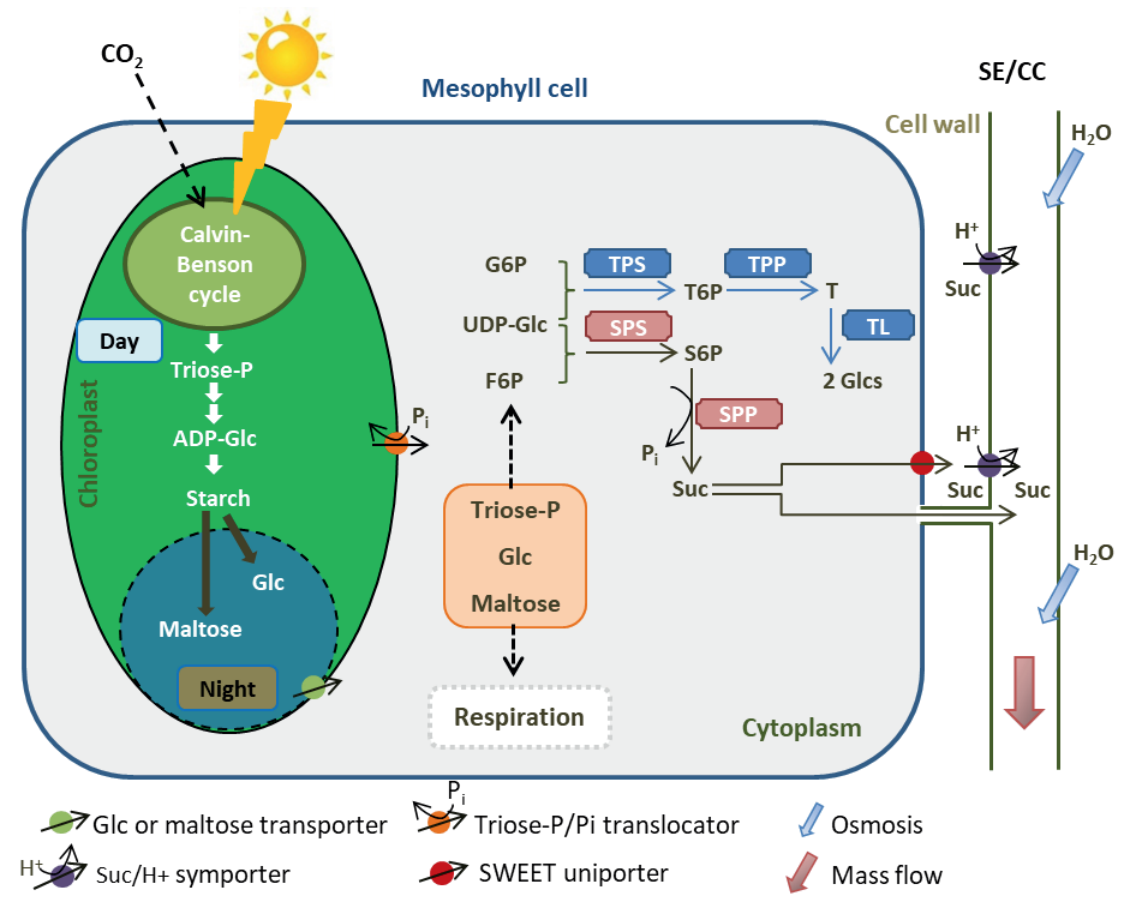

Figure 2. A simplified schematic of sucrose (Suc) synthesis and phloem loading in source leaves. Source leaves use solar energy to fix $\mathrm{CO}_{2}$ to produce triose-phosphate (triose-P) in the chloroplast. Triose-P is then exported to the cytoplasm as precursors for other processes. It may also be converted to ADP-glucose (ADP-Glc) for starch biosynthesis within the chloroplast. At night, starch is degraded into glucose (Glc) or maltose for export to the cytoplasm. Glc is phosphorylated by hexokinase to form Glc-6-phosphate (G6P), which is then converted to fructose-6-phosphate (F6P) by Glc-6-P isomerase. Suc-phosphate synthase (SPS) uses F6P and UDP-Glc as substrates to produce Sucphosphate (Suc-P), which is subsequently converted to Suc by Suc-phosphate phosphatase (SPP). The unloading of Suc into the phloem is either symplastically, i.e. via plasmodesmata (PDs) connecting the sieve element/companion cell (SE/CC) complex, or apoplastically, i.e. via efflux into and uptake from the apoplasm of the SE/CC complex, mediated by the respective sugar transporters. The accumulation of Suc in phloem attracts water osmotically, creating high turgor pressure, which drives mass flow of assimilates toward sinks. Along with the Suc synthesis, a small amount of trehalose (T) may also be synthesized via the reaction with trehalose-6-phosphate (T6P) synthase (TPS) and T6P phosphatase (TPP). TPS catalyzes the transfer of glucose from UDP-glucose to glucose 6-phosphate forming trehalose 6-phosphate (T6P) and UDP, while TPP dephosphorylates T6P to trehalose and inorganic phosphate $(\mathrm{Pi})$. Trehalose may be eventually hydrolyzed by trehalase (TL) into two molecules of Glc. Several transporters involved are also shown. This figure is adapted from Ruan (2014). 


\section{Sucrose phloem loading and transport from source leaves to sink organs}

A majority of the vascular plants use sucrose as main form of carbohydrate for long distance transport. The produced sucrose in the cytosol of mesophyll cells (MC) can be transported via the phloem to different sink organs such as young leaves, roots, and storage organs such as seeds, tubers and fruits. However, the amount of sucrose ready to be translocated depends on several factors: i. photosynthetic activity, ii. destiny of triose-P as mentioned above, and iii. transient storage of sucrose in the vacuole of source leaves (Bornke and Sonnewald 2011). Therefore the source-sink balance will be altered according to the available amount of sucrose for export. Suc loading via phloem can occur either symplastically or apoplastically (Turgeon and Ayre 2005) (Fig. 2). In symplastic active phloem loading, sucrose diffuses from MC to phloem via plasmodesmata (PD) connecting MC to phloem parenchyma cells (PP) and then companion cells. This pathway is also known as polymer trapping approach, where Suc is converted to larger oligosaccharides such as raffinose. This conversion to larger oligosaccharides ensures that sucrose cannot diffuse back to PP via PD. The larger oligosaccharides are then transferred and accumulated in the sieve tube of phloem ready for transport. In apoplastic active phloem loading, sucrose loading to the phloem is transferred through the apoplast between PP and $\mathrm{CC}$, which involves the coordination of several membrane bound proteins such as a sucrose transporter SWEET (Braun 2012), a proton/ sucrose co-transporter SUT1/SUC2 type (Bürkle et al. 1998), a proton $\mathrm{H}^{+} /$pumping ATPase (Michelet and Boutry 1995), and a potassium channel regulator AKT2/3 type (Deeken et al. 2002, Pilot et al. 2003). Both of these active phloem loading approaches create higher solute concentration in the sieve element-companion cell (SE/CC) complex than in the surrounding tissues, which results in a gradient of sucrose concentration within the phloem driving sucrose transport. Collection phloem and release phloem are termed for the phloem zones with higher and lower solute concentrations respectively, while the middle part between these two zones is called transport phloem (Van Bel 2003). The sucrose transport in the phloem is also known as mass-flow as proposed by Ernst Münch in 1930 and widely accepted by other researchers (Knoblauch and Peters 2010, 2013). 


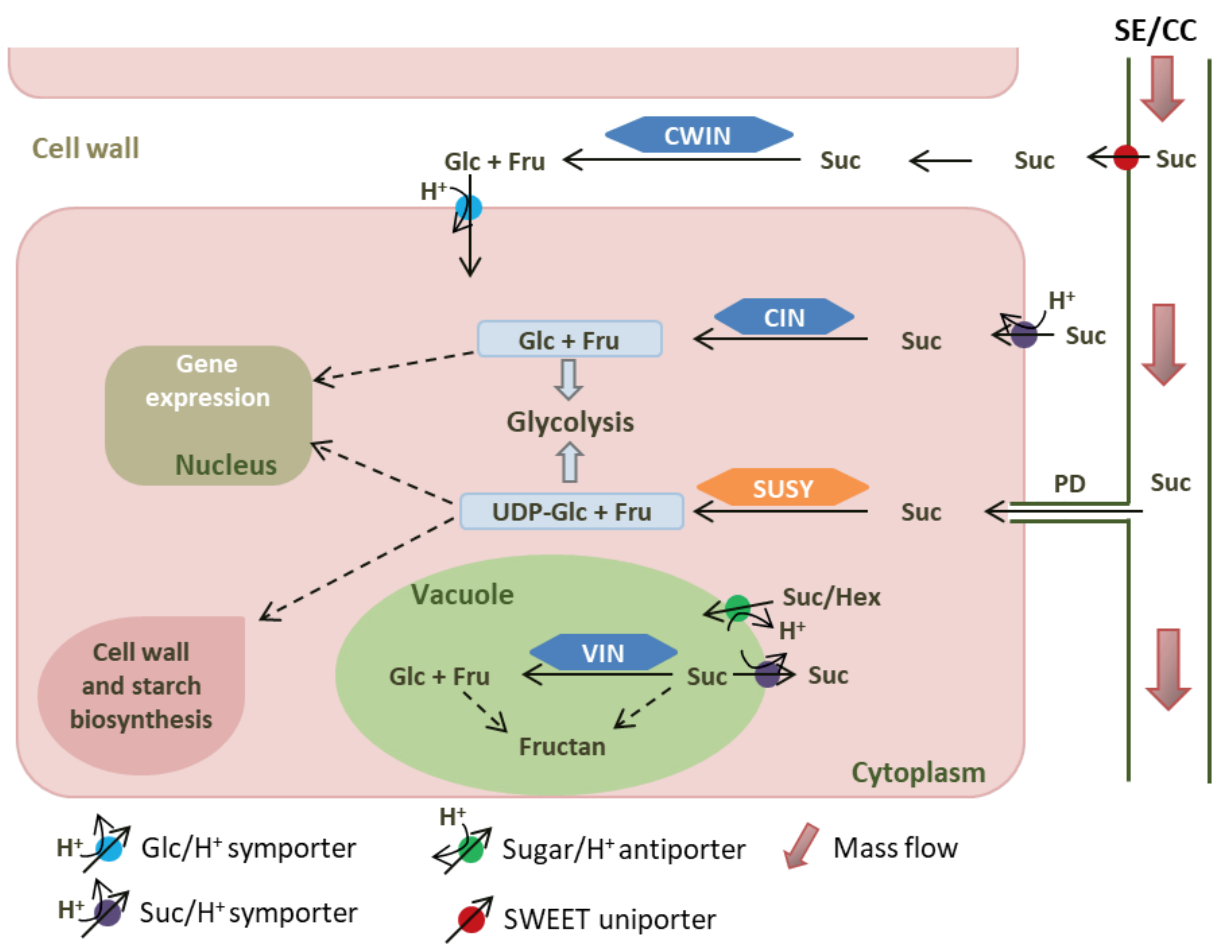

Figure 3. A simplified schematic of sucrose (Suc) unloading, transport, and metabolism in sink tissues. The unloading of Suc from the phloem into sink cells occurs either symplastically or apoplastically. In the symplasic pathway, Suc is imported via plasmodesmata (PDs). In the apoplastic pathway, Suc could be hydrolyzed by cell wall invertase (CWIN) into glucose (Glc) and fructose (Fru) which might be subsequently transported into cytoplasm. Suc could also be taken up by Suc transporters and degraded by cytoplasmic invertase (CIN) and Suc synthase (SUSY). Suc in cytoplasm may also be transported into vacuoles for hydrolysis by vacuolar invertase (VIN). The intracellular hexoses (Glc and Fru) and UDP-Glc are used for glycolysis and synthesis of sugar polymers such as starch, cellulose, and fructan. The levels of these sugars inside the cells influence the gene expression in response to the plant growth and development, as well as to different stresses. Several transporters involved are also shown. Additional abbreviation: SE/CC, sieve element/companion cell complex. This figure is adapted from Ruan (2014).

\section{Sucrose unloading and metabolism in sink organs}

After being transported from the collection phloem, sucrose unloading takes place in the release phloem. Similar to sucrose phloem loading, sucrose unloading in sink tissues occurs either symplastically or apoplastically. Depending on the crop and developmental stage, one 
of these two sucrose unloading pathways is used or both might operate sequentially (Braun et al. 2014). For instance, the sucrose unloading in apples was reported to be apoplastic throughout fruit development (Zhang et al. 2004). Meanwhile Ruan and Patrick (1995) observed symplastic phloem unloading in the early stage of starch accumulation in tomato fruit pericap, and apoplastic phloem unloading in later stage of hexose accumulation in tomato fruit. Unloaded sucrose in sink organs is degraded by either invertase (INV) or sucrose synthase (SUSY). The newly formed products derived from the breakdown of sucrose, e.g. fructose, glucose, UDP-glucose, are used in various metabolic, biosynthetic, and signaling pathways (Fig. 3) (Ruan 2014). Both SUSY and INV play crucial roles in maintaining the carbohydrate metabolism in sink organs and are key in determining sink strength, plant growth, development, and final yield. The plant sink strength is defined as the competitive ability of an organ to import photo-assimilates from source leaves (Ho 1996, Marcelis 1996). The following sections describe the important roles of INV and SUSY in carbohydrate metabolism in sink organs and final performance of the plants in more detail.

\section{Invertases}

Invertase catalyzes an irreversible hydrolysis of sucrose into glucose and fructose (also known as hexoses). These hexoses are then converted into hexose-phosphates by hexokinase and fructokinase using ATP as energy donors (Renz et al. 1993), which are subsequently used in glycolysis or other metabolic processes. Invertases are classified in different isoenzymes according to their optimum $\mathrm{pH}$ and subcellular locations. There are cell wall invertase (CWIN), vacuolar invertase (VIN), and cytoplasmic invertase (CIN) with pH optimum of $\mathrm{pH} 3.5$ - 5.0, pH 5.0 - 5.5, and $\mathrm{pH} 6.8$ - 8.0, respectively (Roitsch and González 2004). CWIN and VIN are glycosylated proteins and belong to glycoside hydrolase family 32 (GH32), whereas CIN is non-glycosylated and classified in family GH100 (Lammens et al. 2009; http://www.cazy.org/). Within each isoenzyme, there is a varying number of genes. For instance, CWIN has four genes in Arabidopsis and tomato (Fridman and Zamir 2003, De Coninck et al. 2005). Meanwhile CIN has nine and six genes in Arabidopsis and rice respectively, and VIN has two genes in Arabidopsis and tomato (Ruan 2014). It should be mentioned that only a few of the genes have been validated as being required for plant development with mutational and gene silencing studies. 


\section{Cell wall invertase (CWIN)}

Cell wall invertases (also known as insoluble acid invertase) play an important role in the regulation of carbohydrate partitioning in sucrose-importing tissues. The expression level of CWIN has been shown to be correlated to the sucrose phloem unloading mode in tomato fruit, i.e. lower gene expression levels at the early stage of fruit development with symplastic unloading and higher expression at the later stages when plants accumulate mostly hexoses with apoplastic unloading (Ruan and Patrick 1995, Jin et al. 2009). The role of CWIN is crucial in sink tissues such as developing seeds where apoplastic unloading is required because of the lack of plasmodesmata. This was evident in the miniature phenotype of maize seeds where a mutation in ZmCwin2 led to lower CWIN activity (Cheng et al. 1996). Several studies have shown that the transcript of Lin5 encoding tomato CWIN1 and Gif1 encoding rice CWNI2 are more abundant in the vascular tissues of developing fruits and rice grains of cultivated accessions, whereas they are absent or present at very low level in the wild accessions with low soluble sugar content in fruit and grain yield, respectively (Kortstee et al. 2007, Wang et al. 2008). The important role of CWIN in fruit soluble sugar content has been confirmed in a tomato introgression line carrying wild Lin5 allele (Fridman et al. 2000, Fridman et al. 2002, Fridman et al. 2004). Those authors showed that the high activity of CWIN encoded by the wild introgressed Lin5 gene in the commercial tomato background is directly correlated with the sharp increase in fruit soluble sugar content. Furthermore, the 3D structure of CWIN has been resolved in Arabidopsis (Verhaest et al. 2006). AtCWIN1 (PDB 2ac1, 537 amino acids) has two main domains: an N-terminal fivefold $\beta$-propeller domain (337 aa), and a C-terminal domain created by two $\beta$-sheets (200 aa). In addition, AtCWIN1 has only two $\alpha$-helices and four $3{ }_{10}$-helices locating within the two main domains. The active site of AtCWIN1 where sucrose binding takes place is composed of the following residues Asp-23, Asp-149, and Glu-203, Lys-242 and Asp-239 (Verhaest et al. 2006, Lammens et al. 2008, Mátrai et al. 2008).

\section{Vacuolar invertase (VIN)}

VIN (also known as soluble acid invertase) plays a crucial role in hexose accumulating tissues. Similar to CWIN, VIN is most active in sink tissues. Studying carbohydrate metabolism in early fruit development of different tomato types, Kortstee et al. (2007) observed that a profound increase in hexose content in developing fruits with higher gene expression and VIN activity in hexose accumulating type, whereas the opposite was reported in the fruits of the sucrose accumulator types. Furthermore, Zrenner et al. (1996) showed that 
cold-induced tubers of the antisense potato with lower VIN activity had a significant decrease in hexose/sucrose ratio compared to the control. Besides regulating hexose content in sink tissues, VIN is also thought to have a direct effect on cell expansion by doubling the osmotic pressure via the hydrolysis of sucrose into hexoses. This effect was evident as a marked decrease in the level of hexoses observed in the reduced tap root size of transgenic carrots with lower VIN activity via antisense approach (Tang et al. 1999).

\section{Cytosol invertase (CIN)}

Cytosol invertase (also known as neutral/alkaline invertase) was first identified to be located in the cytosol only, but later it was found to be present also in other subcellular compartments. A few examples include the work of Vargas et al. (2008) showing that a neutral/alkaline invertase was present in the chloroplasts of Arabidopsis and spinach. In addition, CIN is reported to be involved in root and reproductive development. Barrat et al. (2009) showed that the AtCinv1/AtCinv2 double mutant with $40 \%$ reduced CIN activity had a marked reduction in root growth with abnormal cell expansion. Reduction in root growth and impairment in pollen development and flowering were also observed in the rice mutant with reduced CIN activity (Jia et al. 2008). On the other hand, CIN is thought to be involved in the degradation of sucrose in the cytosol when activity of SUSY is very low (Winter and Huber 2000).

\section{Sucrose synthase}

Sucrose synthase (SUSY) is classified as a retaining glycosyltransferase belonging to family GT4 (http://www.cazy.org/). It catalyzes a reversible conversion of sucrose and UDP to UDP-glucose and fructose (Geigenberger and Stitt 1993). The UDP-glucose can be used directly for cellulose biosynthesis or can be converted to hexose-phosphate Glu-1-P by UDPGlc pyrophosphorylase (UGPase) using inorganic pyrophosphate (PPi). Compared to INV, SUSY is more energy efficient in delivering hexose-phosphate to glycolysis because it catalyzes the reaction with UGPase requiring only one PPi, whereas two molecules of ATP are needed for INV to perform a similar reaction. Up till now AtSUSY1 (PDB 3S27, 808 amino acids) is the only plant SUSY with a resolved crystal structure (Zheng et al. 2011). It has been shown that AtSUSY1 is a tetrameric protein in its native form, composed of three distinct features: i. an N-terminal domain involved in cellular compartmentation which is sub-divided into a cellular targeting domain (CTD, 116 aa) and an ENOD40 peptide-binding domain (EPDB, 120 aa); ii. a GT-B glycosyltransferase domain (GT-B, 548 aa); and iii. a C- 
terminal extension of 28 aa. Several residues involved in substrate binding have been identified by X-ray diffraction of enzyme crystals in complex with: UDP-glucose, and UDP and fructose. Even though the complex of the enzyme and sucrose is still missing, the residues involved in sucrose binding could be derived from the two complexes AtSUSY1•UDP-glucose and AtSUSY1•UDP-fructose.

\section{Subcellular distribution of SUSY isoforms}

SUSY was first identified in the cytosol (Morell and Copeland 1985, Keller et al. 1988, Winter et al. 1998), but later also found to be associated with other subcellular compartments such as the plasma membrane of developing cotton fiber cells (Amor et al. 1995) and actin filaments in maize (Winter et al. 1998). In addition, the subcellular distribution of SUSY is isoform specific and regulated differently. For instance, in maize isoforms SUS1 and SUSSH1 are found to associate with the plasma membrane (Carlson et al. 1996), whereas SUS2 is observed exclusively in the cytosol (Duncan et al. 2006). It has been shown that the association of maize SUS1 to plasma membrane is induced by the phosphorylation of a conserved Serine residue in the N-terminal part of the protein (Hardin et al. 2004a), as well as by a low $\mathrm{pH}$ and high sucrose concentration (Hardin et al. 2006). In addition the association of maize SUS1 with actin filaments was also reported by Duncan and Huber (2007). It turned out that the sites for regulation of SUS1 binding to plasma membrane and actin filaments are different, because high sucrose concentration in the cell induced the association of SUS1 with the plasma membrane (Hardin et al. 2006) but inhibited the association of SUS1 with actin filament (Duncan and Huber 2007). In tobacco pollen tubes SUSY is also found to associate with the cell wall by forming a multiprotein complex involved in cellulose/callose biosynthesis (Persia et al. 2008, Cai et al. 2011).

\section{Expression and regulation of different SUSY genes}

Several SUSY genes have been identified in different crops and their expressions are shown to be specific to specific cell types, tissue types and developmental stages. For instance, out of the six genes identified in Arabidopsis, AtSusy1, AtSusy5, and AtSusy6 are generally expressed in a wide range of organs, while AtSusy2 and AtSusy4 are highly express in seeds and roots, respectively (Baud et al. 2004, Bieniawska et al. 2007). Interestingly, it was reported that SUSY is not required for normal plant growth in Arabidopsis with a Susy1/Susy2/Susy3/Susy4 quadruple mutant lacking SUSY activity in all cell types except the phloem (Barratt et al. 2009). However, Baroja-Fernández et al. (2012) challenged that 
finding, and showed that SUSY activity is still important for cellulose and starch biosynthesis in Arabidopsis under unfavorable conditions. In crop plants, different numbers of Susy genes have been identified, e.g. three in maize (Duncan et al. 2006), six in tomato (Wang et al. 1993b, Chengappa 1998, Goren et al. 2011, Qin et al. 2016), and seven in rice and cotton (Cho et al. 2011, Chen et al. 2012). Chen et al. (2012) reported differential expression patterns of seven Susy genes in distinct tissues and in fibers at different developmental stages in cotton. Those authors observed that Susyl is expressed highest in petals and fibers at 15 days post anthesis (dpa), while Susy3 transcripts are highly abundant in petals and ovules at 0 dpa and also in fibers at $15 \mathrm{dpa}$. Susy4 expression level is very low in fibers but higher in stems, leaves and petals. Similar to the expression of Susyl and Susy3, Susy5 transcripts level is also highest in fibers at $15 \mathrm{dpa}$, while it is low in other tissues like roots, leaves and petals. In contrast, the authors observed that Susy6 is expressed in all the studied tissues but relatively low in fibers. Meanwhile Susy7 expression is only found in stems and petals with low and high level, respectively. Susy2 was the only gene with an undetectable level of transcripts in all the studied tissues in cotton.

\section{The role of SUSY in sink strength, plant growth and development}

Several studies have shown that SUSY plays an important role in sink strength, plant growth and development. For example, Zrenner et al. (1995) showed that the plants with reducing Susy expression in the tubers have lower starch accumulation in their developing tubers compared to the wildtypes. In another study, potato plants with overexpressing Susy4 gene were reported to contain significantly higher starch, UDP-Glucose, and ADP-glucose contents in their tubers, higher yield and dry weight per plant compared to the wildtypes (Baroja-Fernández et al. 2009). The authors observed that the increase in those traits in Susy4-overexpressing plants is solely accounted for by the increase of SUSY activity, not by other enzymes involved in starch and sucrose metabolism. In cotton, a fiberless phenotype is observed in transgenic lines with SUSY activity repressed $70 \%$ or more in the ovule epidermis (Ruan et al. 2003). The authors also reported that the seeds with suppressed SUSY activity in seed coat, endosperm and embryo are shrunken with loss of the transfer cells, and less than $5 \%$ of wildtype seed weight. In tomato, SUSY was deemed as a sink strength indicator during the rapid fruit growth phase, not the fruit ripening phase (Sun et al. 1992). In this study, the authors observed the correlation of SUSY activity and the tomato fruit size during the fruit growth to near full size using a wild tomato species and several commercial cultivars. A year later, Wang et al. (1993a) also reported similar findings that SUSY activity, 
and not acid invertase, is correlated positively with tomato fruit relative growth rate and with starch content in pericarp tissue in fruits at 0 to 39 days post anthesis (dpa). Yet, the evidence was not consistent with experiments using a transgenic approach to confirm the role of SUSY in sink strength. Tomato plants with a reduction in Susyl (also known as TOMSSF) expression are reported to have decreased sucrose import in fruits at $7 \mathrm{dpa}$, but not during the later starch-accumulation phase in fruits at 22 dpa (D'Aoust et al. 1999). In addition, the authors also observed that the transgenic plants with lowest SUSY activity have a reduced fruit set, which leads to significantly fewer fruits per plant at maturity. However, Chengappa et al. (1999) challenged the role of SUSY in regulating sink strength in tomato young fruits when they observed that there was no clear effects on fruit development, starch and sugar contents in young fruits of transgenic lines with reduced Susyl expression.

\section{From sunlight to biomass production at sub-optimal temperature and the road ahead}

The final biomass production is ultimately determined by many processes involving photosynthesis from light harvesting to $\mathrm{CO}_{2}$ fixation, and source-sink relations of carbohydrates for plant growth and development. Depending on the challenges faced by current growth conditions, different approaches can be used to achieve the desirable final biomass production. For instance, it is known that the light in plant canopies in open field fluctuates and cannot be controlled. Therefore the major challenge to improve photosynthesis in plants grown in open field is to efficiently absorb the sunlight to maximize the energy capture, and concomitantly protect itself against the damage of excess sunlight absorption (Horton et al. 1996). It should be noted that this improvement can only be feasible if nonstress conditions for other factors such as $\mathrm{CO}_{2}$, temperature and nutrients are ensured. In the case of protecting itself from the surplus light energy, plants dissipate it as heat via a process called non-photochemical quenching of chlorophyll fluorescence (NPQ) (Müller et al. 2001). However this process often takes long to recover in fluctuating light condition, which transiently reduces the photosynthesis and ultimately decreases up to $21 \%$ of the potential biomass production (Taylor and Long 2017). Kromdijk et al. (2016) demonstrated that this challenge can be overcome with a transgenic approach with the introduction of three genes from Arabidopsis encoding iolaxanthin de-epoxidase, PSII subunit S, and zeaxanthin epoxidase into a tobacco cultivar. Those authors observed that the transgenic lines, having higher transcripts and protein level of those transgenes, had faster recovery of NPQ under 
fluctuating light, higher $\mathrm{CO}_{2}$ uptake and ultimately $15 \%$ increase in dry matter production when compared to the wild type plants. In this thesis, our focus is the challenge of growing tomatoes at sub-optimal temperature (SOT) in the greenhouse. According to Elings et al. (2005), 16\% energy could be saved by a decrease of $2^{\circ} \mathrm{C}$ in temperature set-point. However, it has been shown that young tomato plants grown at SOT have a decrease in the initiation rate of new leaves, the leaf area ratio, and the specific leaf area, leading to a lower relative growth rate (Hoek et al. 1993, Venema et al. 1999b, Heuvelink 2005). In addition, several tomato cultivars exposed to SOT increased leaf soluble sugars and starch content during their vegetative and reproductive phases (Venema et al. 1999b, Kläring et al. 2015). In the reproductive stage, low temperatures cause a delay in the time to flowering and the first fruitharvest (Hurd and Graves 1985, Kläring et al. 2015), a reduction in fruit set due to poor pollen quality (Picken 1984, Zinn et al. 2010), and also parthenocarpic fruits (Adams et al. 2001). In addition, Arabidopsis plants grown at low temperatures have also been shown to have a reduction in biomass yield, which is reported to be due to feedback inhibition of photosynthesis and reduction in sucrose synthesis in the source leaves (Paul and Pellny 2003). This is caused by excessive accumulation of phosphorylated sugars in the source leave, which leads a decrease in photosynthesis due to a drop in free phosphate (Pi) concentration that can be recycled back to the reactions of photosynthesis. This feedback inhibition of photosynthesis results in a reduction of sucrose synthesis in source leaves, less sucrose transported to different sink organs for growth and storage (i.e. weaker sinks), and a decrease in final plant biomass as a consequence. The sucrose metabolism and transport therefore determine the plant sink strength. Alongside with the investigation of phloem transport in tomato and potato using magnetic resonance imaging (MRI) technology within the research program Toward BioSolar Cells, this thesis explored the possibilities to improve tomato sink strength at sub-optimal temperature in order to prevent the feedback inhibition of photosynthesis and ultimately improve biomass production. As described above, sucrose synthases (SUSYs) and invertases (INVs) are the two classes of enzymes that can directly hydrolyse the transported sucrose making them the potential candidates that can be used to improve plant sink strength at sub-optimal temperatures. Sub-optimal temperature (SOT) in this thesis is defined as the growth temperature below the economic optimum temperature 19 $-20^{\circ} \mathrm{C}$ for the tomato cultivation in Dutch greenhouse, but above the threshold temperature causing chilling injury in tomato $\left(12^{\circ} \mathrm{C}\right)$. 


\section{Objectives and outline of this thesis}

As the world population is growing, sustainable crop cultivation is crucial to meet the food and feed demand. As a leading player in agricultural products export, it is important for the Netherlands to maintain this position in a sustainable manner. Among different sectors within agriculture, greenhouse horticulture is an energy intensive sector, in which the energy is used mainly for heating and lighting. As the Netherlands strikes to be a front runner in combatting climate change, the climate act has been proposed to achieve fully carbon neutral electricity by 2050 , and $49 \%$ and $95 \%$ reduction in $\mathrm{CO}_{2}$ emission in 2030 and 2050 compared to the level in 1990 (Goverment of the Netherlands 2016, Groen Links 2018). Therefore along with the development of innovation in technical aspects of greenhouse building materials and cultivation techniques for energy saving, it is also crucial to create tools to breed for new cultivars that are more energy efficient, for instance good performance at lower temperature in the greenhouse. Tomato (Solanum lycopersicum) is the most widely grown crop in Dutch greenhouses and therefore used as the model crop in this thesis.

In Chapter 2 we studied the effects of sub-optimal growing temperature (SOT) on plant growth and development, photosynthetic capacities, biomass allocations using a commercial tomato cultivar S. lycopersicum cv Moneymaker and a high-altitude wild species tomato accession $S$. arcanum LA385 from vegetative phase till fruit production phase which is known to be most sensitive to temperature stress. Furthermore we also investigated the metabolite profiles of both genotypes, such as starch, neutral and phosphorylated sugars, in the source leaves and sink organs- fruits - to investigate whether they were affected differently when grown at SOT, and whether they are linked to plant performance at SOT.

Genetic variation is essential for breeding, which was explored in Chapter 3 with the focus on sucrose synthase (SUSY), a key enzyme of direct sucrose hydrolysis in plants. We explored the natural variation in SUSY by using the availability of genome sequences of 85 accessions from the 150 Tomato Resequencing project (Aflitos et al. 2014). Additionally, we conducted an in silico analysis to predict the potential effects of the identified variation on enzyme functionality. From this, several haplotypes were cloned and heterologously expressed in yeast for further investigating the effect of amino acid substitutions on enzyme kinetics at control and sub-optimal temperatures.

Chapter 4 is a preliminary study investigating the effects of the introgression of Susy3 allele from a high altitude wild accession $S$. neorickii G1.1601 into the cultivated tomato 
Moneymaker background (Susy3-IL) on several parameters, such as growth, biomass allocation, and carbohydrate metabolism, in comparison to the Moneymaker background at control and sub-optimal temperatures. Additionally, we also studied if there was a direct link between gene expression and enzyme activity of different sucrose synthases and invertases and those studied parameters.

Finally the most relevant results found in all above chapters are discussed in Chapter 5. Herein the implications of those findings and the overall prospect of breeding for plants with better performance at lower temperatures are discussed. 



\section{Chapter 2}

\section{High-Altitude Wild Species Solanum arcanum LA385-A Potential Source for Improvement of Plant Growth and Photosynthetic Performance at Suboptimal Temperatures}

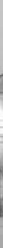

$$
\text { What a }
$$

4

This chapter has been published as:

Dinh Q-D, Dechesne A, Furrer H, Taylor G, Visser RGF, Harbinson J and Trindade LM (2019) High-Altitude Wild Species Solanum arcānum LA385-A Potential Source for Improvement of Plant Growth and Photosynthetic Performance at Suboptimal Temperatures. Front. Plant Sci. 10:1163. doi: $10.3389 /$ fpls.2019.01163

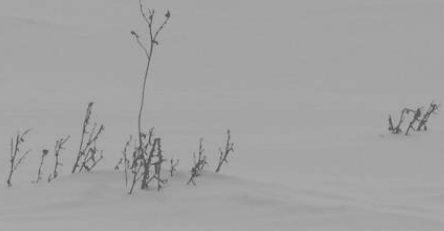




\begin{abstract}
Plant growth, development and yield of current tomato cultivars are directly affected by low temperatures. Although wild tomato species have been suggested as a potential source for low temperature tolerance, very little is known about their behaviour during the reproductive phase. Here we investigated the impact of suboptimal temperatures (SOT, $16 / 14^{\circ} \mathrm{C}$ ), as compared to control temperatures $\left(\mathrm{CT}, 22 / 20^{\circ} \mathrm{C}\right)$, on plant growth, photosynthetic capacity, and carbohydrate metabolism. Under these conditions, two genotypes were analysed: a Solanum lycopersicum cultivar Moneymaker and a high altitude wild species Solanum arcanum LA385, from flowering onset until a later stage of fruit development. Total dry matter production in cv Moneymaker was reduced up to $30 \%$ at SOT, whereas it was hardly affected in wild accession LA385. Specific leaf area, total leaf area, and number of fruits were also decreased at SOT in cv Moneymaker. In contrast wild accession LA385 showed an acclimation to SOT, in which $\Phi_{\mathrm{PSII}}$ and net $\mathrm{CO}_{2}$ assimilation rates were less affected, a similar specific leaf area, higher total leaf area, and higher number of fruits compared to those at CT. In addition, LA385 appeared to have a more distinct sucrose metabolism than cv Monemaker at both temperatures, in which it had higher contents of sucrose-6-phosphate, sucrose, and ratio of sucrose: starch in leaves and higher ratio of sucrose: hexose in fruits. Overall our findings indicate that wild accession LA385 is able to acclimate well to SOT during the reproductive phase, whereas growth and development of cv Moneymaker is reduced at $\mathrm{SOT}$.
\end{abstract}




\section{Introduction}

The growth, development, and yield of crops, such as tomato, are directly influenced by temperature among other climatic factors such as light, water, and humidity. Tomato plants and their fruits can suffer physiological injury when exposed for several weeks to nonfreezing temperature below $12^{\circ} \mathrm{C}$ (Brüggemann et al. 1992, Heuvelink and Dorais 2005). It is reported that the mature tomato plants cannot recover after being exposed to $4^{\circ} \mathrm{C}$ for 10 days (Patade et al. 2018). It is therefore difficult to grow tomatoes commercially as a field crop in the Netherlands and other countries with cool-temperate climate where temperatures below $12^{\circ} \mathrm{C}$ are common in most seasons of the year. In a cool-temperate climate tomatoes are normally commercially grown inside heated glasshouses where growing conditions are tightly controlled for optimal growth, leading to high yields. For example, in the Netherlands the average yield of tomatoes produced in heated glasshouses is about $70 \mathrm{~kg} \mathrm{~m}^{-2} \mathrm{yr}^{-1}$ (Netherlands Foreign Investment Agency 2017). This high yield is obtained at a temperature of $19-20^{\circ} \mathrm{C}$ which is considered to be the economic optimum temperature for heatedglasshouse tomato cultivation in the Netherlands (van der Ploeg and Heuvelink 2005). However this high productivity requires a large energy input, which accounts for one third of the total production cost and raises concerns over the environmental impact of this production system. It is estimated that a temperature decrease of $2^{\circ} \mathrm{C}$ inside the glasshouse would reduce energy use for heating by $16 \%$ (Elings et al. 2005). Therefore, it is desirable to decrease the temperature set-point in the glasshouse so that energy costs and negative environment impacts can be reduced. With modern greenhouse tomato cultivars such a temperature decrease would result in a decrease in plant growth and yield, something which would make the otherwise desirable reduction in energy use economically unsustainable. For greenhouse tomatoes in the Netherlands temperatures below the economic optimum temperature 19 $20^{\circ} \mathrm{C}$, but above the threshold temperature for chilling injury $\left(12^{\circ} \mathrm{C}\right)$, are classified as suboptimal temperatures (SOT).

Suboptimal temperatures are reported to have several negative effects on the growth and development of cultivated tomatoes. It has been shown that SOT decreased the initiation rate of new leaves, the leaf area ratio, and the specific leaf area in young cultivated tomato plants, leading to a lower relative growth rate (Hoek et al. 1993, Venema et al. 1999b, Heuvelink 2005). In addition, several tomato cultivars exposed to SOT increased leaf soluble sugars and starch content during their vegetative and reproductive phases (Venema et al. 1999b, Kläring et al. 2015). In the reproductive stage, low temperatures cause a delay in the time to flowering and the first fruit-harvest (Hurd and Graves 1985, Kläring et al. 2015), a reduction in fruit set due to poor pollen quality (Picken 1984, Zinn et al. 2010), and also parthenocarpic fruits (Adams et al. 2001).

Variation in the SOT response between cultivated tomatoes is limited, and this hinders breeding for improved levels of production at lower temperatures. The limited genetic variation found in cultivated tomatoes for low temperature tolerance could be augmented by using germplasm from high altitude wild tomatoes species (Venema et al. 1999a, Venema et al. 1999b, Foolad and Lin 2000, Venema et al. 2005, van der Ploeg et al. 2007a, van der Ploeg et al. 2007b). While high altitude tomato accessions have been shown to have better 
low temperature tolerance, all these studies have been carried out with young plants in the vegetative growth stage, even though the reproductive phase of tomatoes is known to be the most temperature-sensitive growth stage (Zinn et al. 2010). The objective of the study we report here was therefore to investigate the differential responses to SOT of photosynthesis, growth, and carbohydrate distribution between different plant organs from flowering onset until fruit harvest between a cultivated and a high altitude wild species. The findings from this study provides new insights into how this wild tomato $S$. arcanum LA385 copes with SOT in those aspects during the reproductive stage. The knowledge from this study would bring us one step closer to exploit this wild tomato as the source to breed for new cultivar performing well at SOT, which would ultimately help the tomato greenhouse cultivation in temperate regions reduce energy consumption and be more sustainable.

\section{Materials and methods}

\section{Plant materials}

Two genotypes were used in this study: a cultivated tomato Solanum lycopersicum cv. Moneymaker (Moneymaker or MM) and a high altitude S. arcanum LA385 (LA385). The seeds were obtained from Centre of Genetic Resources, Wageningen, the Netherlands. Cultivar Moneymaker is an indeterminate and self-compatible tomato type. It is a popular English greenhouse variety which was first released in 1913 by F. Stonor of Southampton (Everwilde Farms Inc. 2019). Due to its popularity, Moneymaker has been frequently used as a recurrent parent together with relative tomato accessions as donor parents in many backcross populations, to improve traits such as fruit quality or resistance to plant diseases (Voorrips et al. 2000, Finkers et al. 2008, Víquez-Zamora et al. 2014a, Barrantes et al. 2016). S. aracunum LA385 is a wild species tomato, originally collected on 1956 at San Juan in Peru, approximately at $2,500 \mathrm{~m}$ above sea level, and maintained by Tomato Genetics Resource Center (http://tgrc.ucdavis.edu/). LA385 is an indeterminate and allogamous selfincompatible type. It produces small green fruits. This high altitude wild species was chosen in this research because it has been shown to be tolerant to SOT at the vegetative stage (Venema et al. 1999b), but was not studied at the reproductive stage where plants are most sensitive to temperature stress.

\section{Growth conditions and treatments}

Tomato seeds were sown in small pots with commercial potting soil (ED73, Einheitserde, Sinntal-Altengronau, Germany) and germinated seedlings grown for two weeks in the glasshouse and then transplanted into separate $17 \mathrm{~cm}$ pots containing the same soil. The plants were watered twice a week. After four weeks these plants were moved into two climate chambers. The climate chambers' growing conditions were: $16 / 8 \mathrm{~h}$ day/night regime $(06.00-$ $22.00 \mathrm{~h}$ day and $22.00-06.00 \mathrm{~h}$ night), $22 / 20^{\circ} \mathrm{C}$ day/night temperature, an irradiance of 300 $\mu \mathrm{mol} \mathrm{m} \mathrm{m}^{-2} \mathrm{~s}^{-1}$ during the day provided by a mixture of $50 \%$ mercury (Master HPI-T Plus, $400 \mathrm{~W}$; Philips, Eindhoven, the Netherlands) and sodium vapor high pressure lamps (SON-T Agro, $400 \mathrm{~W}$; Philips), $400 \mathrm{ppm} \mathrm{CO}_{2}$, and an average relative humidity of $70 \%$. This will be 
referred to as 'control temperature' (CT). The plants were acclimated to the CT for one week. From week 5 after sowing, the temperature regime of one climate chamber was changed to $16 / 14^{\circ} \mathrm{C}$ day/night while the other parameters remained the same as in CT chamber, and this will be referred to as 'suboptimal temperature' (SOT). This SOT regime was chosen because it is below the current optimum temperature $19-20^{\circ} \mathrm{C}$ for heated glasshouse tomato cultivation in the Netherlands, and well above the non-freezing temperature of $12^{\circ} \mathrm{C}$ which is known to cause physiological injury to tomato plants and their fruits when exposed for several weeks (Brüggemann et al. 1992, Heuvelink and Dorais 2005). Plants were given nutrient solution A (1:100 dilution, 20-5-10-2 N-P-K-Mg; Hakaphos, Scotts, $\mathrm{OH}$ ) weekly during the first five weeks, and three times per week from the sixth week onward with nutrient solution $\mathrm{B}$ (Supplementary Table 1).

This study consisted of two trials. Trial A was from the onset of flowering phase until the young fruit stage, which lasted for nine weeks from mid-January until the end of March 2015. Trial B was from the stages of young to mature fruit development, which lasted for 16 weeks from beginning of October 2014 until the end of January 2015. Moneymaker is selfcompatible, so the pollination of its flowers was done by gently tapping on the base of fluorescence. Meanwhile $S$. arcanum LA385 is self-incompatible, the plants in trial B were sib pollinated when 75 days old in CT (week 11) and at 68 days old (week 10) in SOT.

\section{Analysis of growth and development}

Total leaf area, number of fruits, fresh weight and dry weight of leaves, stems, fruits, and roots were measured every two weeks. Total leaf area was measured by a leaf-area meter (model 3100, Li-Cor Inc., Lincoln, NE, USA). Relative leaf thickness was calculated as the division of the total leaf area by leaf fresh weight (Medek et al. 2007). After fresh weight was measured, a small portion of each tissue was collected and snap-frozen in liquid nitrogen and stored at $-80^{\circ} \mathrm{C}$ for further measurements of soluble and phosphorylated sugars and starch contents. The harvested leaves, stems, fruits, and roots were oven-dried at $105^{\circ} \mathrm{C}$ for 24 hours, and then the dry weight was measured.

\section{CO2 exchange and chlorophyll fluorescence measurement}

$\mathrm{CO}_{2}$ exchange and chlorophyll fluorescence were measured with an open gas-exchange system Li-Cor 6400 (Li-Cor Inc., Lincoln, NE, USA) equipped with an integrated $2 \mathrm{~cm}^{2}$ fluorescence chamber head (Li-6400-40). All measurements were made on the youngest fully expanded leaf after the plants have been in the light for at least 3 hours, and between $09.00-$ $17.00 \mathrm{~h}$. The response of assimilation to irradiance was measured at $400 \mathrm{ppm} \mathrm{CO}_{2}$, a gas flow rate of $400 \mathrm{~mol} \mathrm{~s}^{-1}$, a leaf temperature of $22^{\circ} \mathrm{C}(\mathrm{CT})$ or $16^{\circ} \mathrm{C}$ (SOT) and a range of irradiances starting from zero up to a saturating irradiance (in some cases an irradiance of $2000 \mu \mathrm{mol} \mathrm{m}$ $\mathrm{s}^{-1}$ ). In addition, two chlorophyll fluorescence based parameters for each leaf were measured. The maximum quantum efficiency of photosystem II (PSII) photochemistry (Fv/Fm) was measured for each leaf, and the PSII operating efficiency $\left(\Phi_{\mathrm{PSII}}\right)$ was measured for each leaf at each irradiance step. These parameters were calculated using equations 1 and 2: 


\section{Equation $1 \quad \mathrm{Fv} / \mathrm{Fm}=(\mathrm{Fm}-\mathrm{Fo}) / \mathrm{Fm}$}

where Fv, Fm, Fo are the variable (i.e. Fm - Fo), maximum, and minimum fluorescence yields measured from 20min dark-adapted leaf;

Equation $2 \quad \Phi_{\mathrm{PSII}}=\left(\mathrm{Fm}^{\prime}-\mathrm{Fs}\right) / \mathrm{Fm}^{\prime}$

where Fm' and Fs are the maximum and steady state fluorescence yields of light-adapted leaves (Baker et al. 2007).

\section{Soluble sugars and starch content measurement}

Soluble sugars and starch content were measured on the youngest mature leaves and fruits. The extraction of soluble sugars and starch was adapted from the protocol of Kortstee et al. (2007) with some modifications. Twenty milligrams of freeze-dried sample were extracted three times for $40 \mathrm{~min}$ with $1 \mathrm{~mL}$ of $80 \%$ ethanol at $80^{\circ} \mathrm{C}$. After each extraction, insoluble material was pelleted by centrifugation $(10000 \times \mathrm{g}$ for $10 \mathrm{~min})$ and the supernatant was transferred to a new tube. The pellet was kept for starch content measurement. The protocol of soluble sugar content measurement was performed essentially described in Rashidi and Trindade (2018). Five hundred microliters of the supernatant was dried by vacuum centrifugation with a RapidVap (Labconco, Kansas city, MO, USA), and subsequently resuspended in $500 \mu \mathrm{L}$ of MilliQ water (Merck, USA). The clear supernatant was used to measure soluble sugars using HPAEC-PAD Dionex ICS5000+ DC equipped with a Dionex CarboPac PA1 column $(2 \times 250 \mathrm{~mm})$ preceded by a similar guard column $(2 \times 50 \mathrm{~mm})$. The flow rate was $0.25 \mathrm{ml}$ per min and 2.51 of sample were injected using a Dionex AS-AP autosampler. The elution program consisted of an isocratic elution of $10 \mathrm{mM}$ sodium hydroxide in $25 \mathrm{~min}$. Each run was followed by a $5 \mathrm{~min}$ wash with $1 \mathrm{M}$ sodium acetate in 100 $\mathrm{mM}$ sodium hydroxide and a 30 min equilibration with $10 \mathrm{mM}$ sodium hydroxide prior to the next injection. The eluent was monitored by a thermostatic Thermo Scientific ICS5000 pulsed electrochemical detector (PAD). The samples were cooled to $5^{\circ} \mathrm{C}$, the Dionex ICS5000+DP column oven temperature was set at $30^{\circ} \mathrm{C}$. A series of standard neutral sugars with known concentrations was also run and used for the calculation. The output was subsequently processed with software ChromeleonTM Chromatography Data System version 7 (Thermo Scientific, USA).

Starch content from leaves and fruits was determined using a starch assay kit (No. 0207748, Boehringer, Mannheim, Germany). Briefly, the pellet was solubilized in a solution containing $0.5 \mathrm{~mL}$ of $8 \mathrm{M} \mathrm{HCl}$ and $2 \mathrm{~mL}$ of DMSO and then incubated at $60^{\circ} \mathrm{C}$ for $1 \mathrm{~h}$. The mixture was subsequently neutralized with $0.5 \mathrm{~mL}$ of $5 \mathrm{M} \mathrm{NaOH}$ and $7 \mathrm{~mL}$ of $0.1 \mathrm{M}$ citrate buffer $\mathrm{pH}$ 4.6. The insoluble material was pelleted by centrifugation $(10000 \mathrm{x} g$ for $1 \mathrm{~min})$. Ten microliters of the clear supernatant was used to determine the starch content. 


\section{Measurement of phosphorylated sugars}

Phosphorylated sugars were extracted from $30 \mathrm{mg}$ of freeze-dried leaves using the protocol of De Bruijn et al. (1999) with some modifications. Glucose-1,6-bisphosphate was used as the internal standard for phosphorylated sugars. All the standard phosphorylated sugars used in this study, i.e. sucrose-6-phosphate (Suc-6-P), glucose-1-phosphate (Gluc-1-P), glucose-6phosphate (Glu-6-P), mannose-6-phosphate (Man-6-6P), and fructose-6-phosphate (Fru-6-P), were purchased from Sigma (USA). HPAEC-PAD was used to determine the phosphorylated sugar content and was performed on a Dionex ICS5000+ DC equipped with a Dionex CarboPac PA1 column $(2 \times 250 \mathrm{~mm})$ proceeded by a similar guard column $(2 \times 50 \mathrm{~mm})$. The flow rate was $0.25 \mathrm{ml} \mathrm{min}$ m $^{-1}$ and 1 of sample was injected using a Dionex AS-AP autosampler. The elution program consisted of a gradient of sodium acetate from 50 to 800 $\mathrm{mM}$ in $40 \mathrm{~min}$ in an isocratic background of $4 \mathrm{mM}$ sodium hydroxide. Each run was followed by a $5 \mathrm{~min}$ wash with $800 \mathrm{mM}$ sodium acetate and a 15 min equilibration with $50 \mathrm{mM}$ sodium acetate, both in $4 \mathrm{mM}$ sodium hydroxide, prior to the next injection. The eluent was monitored by a temperature controlled Thermo Scientific ICS5000 pulsed electrochemical detector (PAD). The samples were cooled to $5^{\circ} \mathrm{C}$ and the Dionex ICS5000+DP column oven was cooled to $10^{\circ} \mathrm{C}$. A series of standard neutral sugars with known concentrations was run through the HPLC/detector system to calibrate the assays. The output was subsequently processed with the same software as used in the neutral sugar measurements above.

\section{Statistical analysis}

One-way analysis of variance (ANOVA) was used to evaluate statistically significant effect of temperature on different parameters aforementioned. We also used two-way ANOVA to evaluate the combined effects of the genotypes and growth temperatures on those same parameters. Each parameter was measured in three to five individual plants. Statistical analyses were carried out with GenStat $17^{\text {th }}$ edition (VSN International Ltd, UK). 


\section{Results}

\section{Plant growth and dry matter allocation is affected by suboptimal temperature}

Suboptimal temperature strongly affected plant growth and dry matter allocation in the tomato cultivar Moneymaker, but less so in wild accession LA385 (Table 1 and Table 2). The total leaf area (TLA), specific leaf area (SLA), total fresh weight, total dry matter, and number of fruits in Moneymaker were significantly reduced at SOT compared to those at CT. In addition, the first fruit harvest in Moneymaker was delayed in SOT (Table 2), and in week 16 its fruits were still immature and green in color while by this stage there were several ripe, red fruits in CT (data not shown). Furthermore, LA385 showed an acclimation of several parameters to SOT, compared to CT. There was an increase in SLA during the later stages of fruit development, a 1.4 fold higher TLA, accompanied by a twofold increase in fruit number, a slight decrease in leaf DM content in week 16, and stable total dry matter (DM) yield (Table 2). At SOT both genotypes had thicker leaves and contained slightly more DM in the leaves than in the roots, which was evident from the higher leaf to root ratio (Table 1 and Table 2, and see also Supplementary Figure 1). Furthermore, DM allocation to different plant organs was also affected at SOT (Figure 1). In both genotypes more DM was allocated to leaves (Fig. 1A,B) and less DM was partitioned to stems (Figure 1C,D) at SOT than at CT. The fraction of DM allocation to roots was not significantly affected by temperature (Figure 1E,F). The DM allocation to fruits in Moneymaker dropped significantly from 34\% at CT to $10 \%$ at SOT in week 16, whereas it remained stable at around 5\% in LA385 (Figure $\mathbf{1 G}, \mathbf{H})$. The interactive effect of temperature and genotypes in many of these parameters were also demonstrated in Supplementary Table 2. 
Table 1: Growth parameters of $S$. lycopersicum cv Moneymaker and wild species $S$. arcanum LA385 at control $\left(22^{\circ} \mathrm{C}\right)$ and suboptimal temperatures $\left(16^{\circ} \mathrm{C}\right)$ in trial $\mathrm{A}$.

\begin{tabular}{|c|c|c|c|c|c|}
\hline & \multirow{2}{*}{$\begin{array}{l}\mathrm{T} \\
\left({ }^{\circ} \mathrm{C}\right)\end{array}$} & \multicolumn{2}{|l|}{ Moneymaker } & \multicolumn{2}{|l|}{ LA385 } \\
\hline & & Week $7(2)^{*}$ & Week 9 (4) & Week 7 (2) & Week 9 (4) \\
\hline \multirow[t]{2}{*}{ Total leaf area $\left(\mathrm{cm}^{2}\right)$} & 22 & $2026 \pm 48$ & $3467 \pm 84$ & $714 \pm 3$ & $1191 \pm 40$ \\
\hline & 16 & $2168 \pm 48$ & $3027 \pm 101^{\mathrm{a}}$ & $741 \pm 14$ & $1083 \pm 29$ \\
\hline Specific leaf area & 22 & $181.3 \pm 4.8$ & $150.9 \pm 3.5$ & $198.6 \pm 3.8$ & $135.3 \pm 6.8$ \\
\hline$\left(\mathrm{cm}^{2} \mathrm{~g} \mathrm{DW}^{-1}\right)$ & 16 & $170.4 \pm 2.8$ & $119.6 \pm 2.0^{c}$ & $170.6 \pm 3.8 \mathrm{c}$ & $104.4 \pm 3.5^{b}$ \\
\hline \multirow{2}{*}{$\begin{array}{l}\text { Relative leaf } \\
\text { thickness }\end{array}$} & 22 & $0.051 \pm 0.002$ & $0.051 \pm 0.001$ & $0.063 \pm 0.009$ & $0.043 \pm 0.001$ \\
\hline & 16 & $0.048 \pm 0.000$ & $0.058 \pm 0.001^{\mathrm{b}}$ & $0.042 \pm 0.001^{\mathrm{a}}$ & $0.049 \pm 0.004$ \\
\hline Total fresh weight & 22 & $150.5 \pm 6.0$ & $322.5 \pm 5.2$ & $67.9 \pm 8.0$ & $120.8 \pm 6.1$ \\
\hline$\left(\right.$ g plant $\left.^{-1}\right)$ & 16 & $144.6 \pm 5.8$ & $252.9 \pm 4.9^{c}$ & $57.1 \pm 2.1$ & $102.7 \pm 5.8$ \\
\hline Total dry matter & 22 & $15.3 \pm 0.4$ & $39.4 \pm 0.5$ & $5.9 \pm 0.2$ & $19.3 \pm 1.5$ \\
\hline$\left(\right.$ g plant $\left.^{-1}\right)$ & 16 & $16.8 \pm 0.7$ & $35.3 \pm 0.8^{b}$ & $7.3 \pm 0.3^{b}$ & $18.4 \pm 1.2$ \\
\hline \multirow{2}{*}{$\begin{array}{l}\text { Leaf dry matter } \\
\text { content }(\%)\end{array}$} & 22 & $11.0 \pm 0.7$ & $13.1 \pm 0.1$ & $8.8 \pm 1.5$ & $17.4 \pm 0.9$ \\
\hline & 16 & $12.3 \pm 0.1$ & $14.5 \pm 0.2^{c}$ & $13.9 \pm 0.1^{\mathrm{a}}$ & $20.0 \pm 1.1$ \\
\hline \multirow{2}{*}{$\begin{array}{l}\text { Leaf to root dry } \\
\text { mater ratio }\left(\mathrm{g} \mathrm{g}^{-1}\right)\end{array}$} & 22 & $5.43 \pm 0.16$ & $5.13 \pm 0.18$ & $2.71 \pm 0.17$ & $2.26 \pm 0.10$ \\
\hline & 16 & $6.24 \pm 0.34$ & $5.72 \pm 0.12^{\mathrm{a}}$ & $3.01 \pm 0.14$ & $2.54 \pm 0.13$ \\
\hline \multirow[t]{2}{*}{ Number of fruits } & 22 & n.a. & $10 \pm 0$ & n.a. & $6 \pm 1$ \\
\hline & 16 & n.a. & n.a. & n.a. & n.a. \\
\hline
\end{tabular}

\footnotetext{
* (n): number of weeks exposed to SOT; n.a.: not available. Data represent the mean of five individual plants $( \pm \mathrm{SE})$. Significant differences are denoted as a, b, and $\mathrm{c}$ for $P<0.05,<0.01$, and $<$ 0.001 , respectively.
} 
Table 2: Growth parameters of S. lycopersicum cv Moneymaker and wild species $S$. arcanum LA385 at control $\left(22^{\circ} \mathrm{C}\right)$ and suboptimal temperatures $\left(16^{\circ} \mathrm{C}\right)$ in trial $\mathrm{B}$.

\begin{tabular}{|c|c|c|c|c|c|}
\hline & \multirow{2}{*}{$\begin{array}{l}\mathrm{T} \\
\left({ }^{\mathrm{o}} \mathrm{C}\right)\end{array}$} & \multicolumn{2}{|l|}{ Moneymaker } & \multicolumn{2}{|l|}{ LA385 } \\
\hline & & Week $9(4)^{*}$ & Week $16(11)$ & Week 9 (4) & Week $16(11)$ \\
\hline Total leaf area & 22 & $2427 \pm 48$ & $5996 \pm 451$ & $711 \pm 57$ & $1568 \pm 99$ \\
\hline$\left(\mathrm{cm}^{2}\right)$ & 16 & $1682 \pm 60^{c}$ & $4345 \pm 328^{a}$ & $707 \pm 48$ & $2149 \pm 123^{b}$ \\
\hline Specific leaf area & 22 & $107.0 \pm 1.0$ & $163.6 \pm 6.3$ & $110.1 \pm 2.4$ & $126.9 \pm 2.7$ \\
\hline$\left(\mathrm{cm}^{2} \mathrm{~g} \mathrm{DW}^{-1}\right)$ & 16 & $89.5 \pm 3.4^{b}$ & $118.9 \pm 9.2^{b}$ & $86.5 \pm 4.4^{b}$ & $123.8 \pm 1.8$ \\
\hline \multirow{2}{*}{$\begin{array}{l}\text { Relative leaf } \\
\text { thickness }\end{array}$} & 22 & $0.052 \pm 0.001$ & $0.051 \pm 0.004$ & $0.040 \pm 0.000$ & $0.044 \pm 0.002$ \\
\hline & 16 & $0.055 \pm 0.001$ & $0.067 \pm 0.006$ & $0.049 \pm 0.001^{b}$ & $0.051 \pm 0.001^{b}$ \\
\hline Total fresh weight & 22 & $343.0 \pm 25.7$ & $1284.6 \pm 64.9$ & $132.9 \pm 2.3$ & $325.2 \pm 14.7$ \\
\hline$\left(\right.$ g plant $\left.^{-1}\right)$ & 16 & $240.4 \pm 8.7^{\mathrm{a}}$ & $644.8 \pm 25.2^{c}$ & $103.9 \pm 3.4^{b}$ & $325.4 \pm 14.4$ \\
\hline Total dry matter & 22 & $60.2 \pm 5.7$ & $128.0 \pm 7.1$ & $21.5 \pm 0.6$ & $54.5 \pm 2.7$ \\
\hline$\left(\right.$ g plant $\left.^{-1}\right)$ & 16 & $42.5 \pm 3.5$ & $83.6 \pm 3.5^{c}$ & $18.6 \pm 1.3$ & $54.0 \pm 2.5$ \\
\hline \multirow{2}{*}{$\begin{array}{l}\text { Leaf dry matter } \\
\text { content }(\%)\end{array}$} & 22 & $17.9 \pm 0.1$ & $12.2 \pm 0.4$ & $22.6 \pm 0.7$ & $18.1 \pm 1.0$ \\
\hline & 16 & $20.5 \pm 1.1$ & $13.1 \pm 0.8$ & $23.6 \pm 0.6$ & $15.9 \pm 0.3$ \\
\hline \multirow{2}{*}{$\begin{array}{l}\text { Leaf to root dry } \\
\text { mater ratio }\left(\mathrm{g} \mathrm{g}^{-1}\right)\end{array}$} & 22 & $1.02 \pm 0.27$ & $1.98 \pm 0.19$ & $0.77 \pm 0.08$ & $0.88 \pm 0.07$ \\
\hline & 16 & $1.18 \pm 0.23$ & $2.60 \pm 0.11^{\mathrm{a}}$ & $1.30 \pm 0.04^{b}$ & $1.44 \pm 0.12^{b}$ \\
\hline \multirow[t]{2}{*}{ Number of fruits } & 22 & $6 \pm 1$ & $26 \pm 2$ & n.a. & $17 \pm 4$ \\
\hline & 16 & n.a. & $18 \pm 2^{a}$ & n.a. & $30 \pm 2^{a}$ \\
\hline
\end{tabular}

\footnotetext{
* (n): number of weeks exposed to SOT; n.a.: not available. Data represent the mean of three individual plants in week 9 and five individual plants in week $16( \pm \mathrm{SE})$. Significant differences are indicated with $\mathrm{a}, \mathrm{b}$, and $\mathrm{c}$ for $P<0.05,<0.01$, and $<0.001$, respectively.
} 


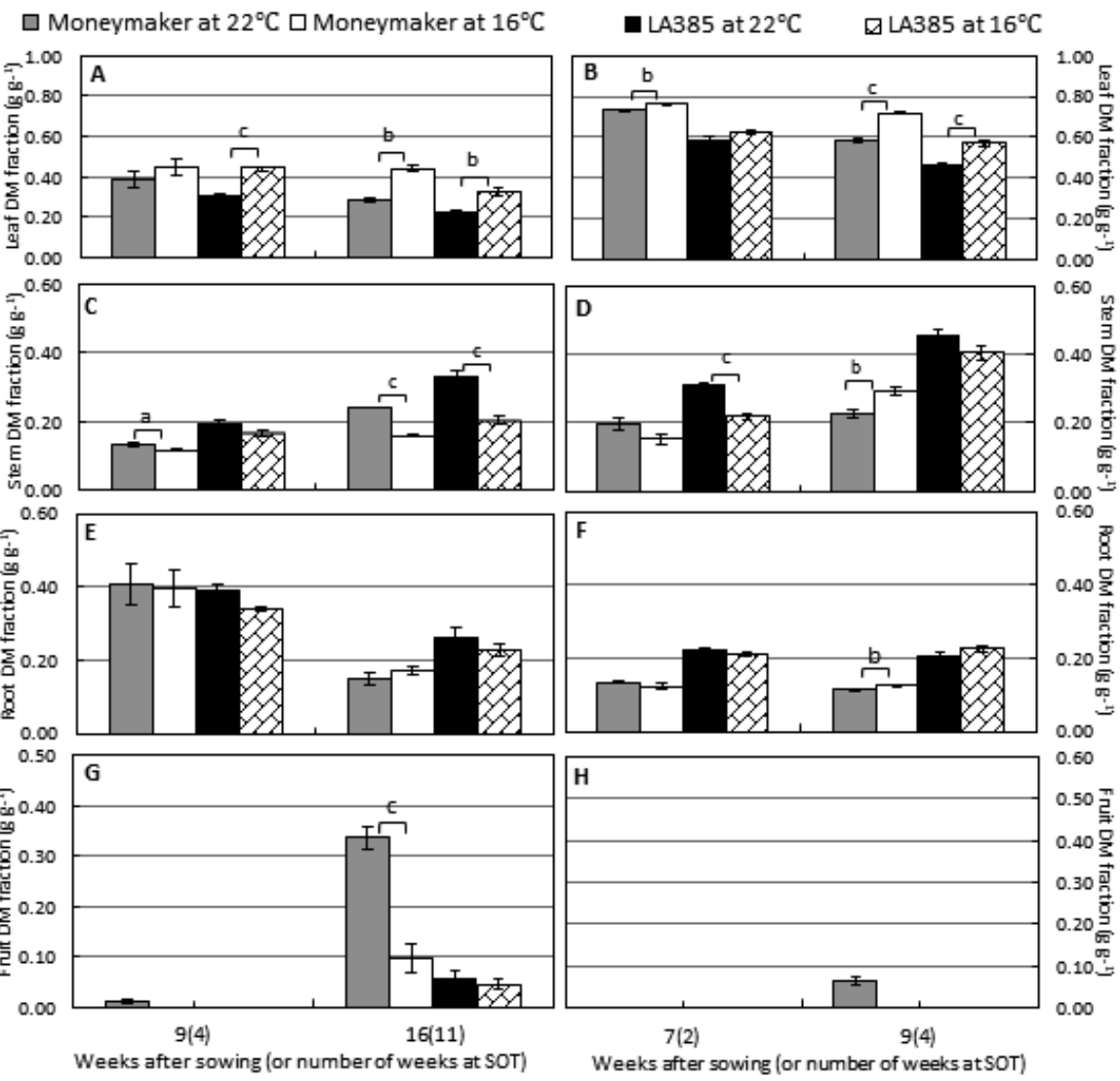

FIGURE 1 | Effect of SOT on dry matter allocation to different tomato organs of cv. Moneymaker and wild accession LA385. DM allocation to leaf, stem, root, and fruit are depicted in (panel $\mathbf{A}$ and B), (panel $\mathbf{C}$ and $\mathbf{D})$, (panel $\mathbf{E}$ and $\mathbf{F}$ ), and (panel $\mathbf{G}$ and $\mathbf{H}$ ), respectively. Panels on the left and right are from growth trial (A) and trial (B), respectively. The error bars represent standard error of fi individual plants $( \pm \mathrm{SE})$, except week 9 of trial (B) with three plants. Significant difference is denoted as a, $\mathrm{b}$, and $\mathrm{c}$ for $\mathrm{P}<0.05,<0.01$, and $<0.001$, respectively. 


\section{Chlorophyll fluorescence parameters and $\mathrm{CO}_{2}$ assimilation are affected by $\mathrm{SOT}$ in $\mathrm{cv}$ Moneymaker but to a lower extent in wild accession LA385}

The maximum quantum efficiency of PSII photochemistry $\left(\mathrm{F}_{\mathrm{v}} / \mathrm{F}_{\mathrm{m}}\right)$ in both genotypes was not significantly affected at SOT, while the PSII operating efficiency $\left(\Phi_{\mathrm{PSII}}\right)$ (measured at 300 $\mu \mathrm{mol} \mathrm{m} \mathrm{m}^{-2} \mathrm{~s}^{-1}$ ) was affected (Table 3 and Supplementary Figure 2). In the case of Moneymaker $\Phi_{\text {PSII }}$ was significantly decreased at SOT compared to CT, with reductions of $26 \%$ and $18 \%$ at week 7 and week 16, respectively. In LA385 $\Phi_{\text {PSII }}$ also decreased at SOT compared to CT, but only by $2-5 \%$. Furthermore, we also observed a positive correlation between $\Phi_{\mathrm{PSII}}$ and net $\mathrm{CO}_{2}$ assimilation rate $\left(\mathrm{A}_{\mathrm{N}}\right)$ (Table 3 and Supplementary Figure 3 ). As with $\Phi_{\text {PSII }}$, the $A_{N}$ (measured at $300 \mu \mathrm{mol} \mathrm{m}^{-2} \mathrm{~s}^{-1}$ ) of both genotypes were affected by SOT compared to CT, but the impact was less significant for LA385 than for Moneymaker, especially at week 16. In addition, the light saturated $\mathrm{CO}_{2}$ assimilation rate $\left(\mathrm{A}_{\text {sat }}\right)$ of Moneymaker was much lower at SOT in both week 7 and 16, whereas $\mathrm{A}_{\text {sat }}$ of LA385 was only slightly affected in SOT compared to CT. The combined effect of temperature and genotype was most clear in $A_{N}$ at week 7 and in $\Phi_{\text {PSII }}$ at both week 7 and 16 (Supplementary Table 2).

Table 3: Influence of SOT on Fv/Fm, net $\mathrm{CO}_{2}$ assimilation rate $\left(\mathrm{A}_{\mathrm{N}}\right)$ and $\Phi_{\mathrm{PSII}}$ at PFD of $300 \mu \mathrm{mol} \mathrm{m}$ ${ }^{2} \mathrm{~s}^{-1}$, and light saturated $\mathrm{CO}_{2}$ assimilation rate $\left(\mathrm{A}_{\mathrm{sat}}\right)$.

\begin{tabular}{|c|c|c|c|c|c|}
\hline & \multirow{2}{*}{$\begin{array}{l}\mathrm{T} \\
\left({ }^{\circ} \mathrm{C}\right)\end{array}$} & \multicolumn{2}{|c|}{ Moneymaker } & \multicolumn{2}{|c|}{ LA385 } \\
\hline & & Week 7 (2)* & Week $16(11)$ & Week 7 (2) & Week 16 (11) \\
\hline \multirow[t]{2}{*}{$\mathrm{Fv} / \mathrm{Fm}$} & 22 & $0.810 \pm 0.001$ & $0.806 \pm 0.006$ & $0.820 \pm 0.003$ & $0.816 \pm 0.006$ \\
\hline & 16 & $0.804 \pm 0.001^{b}$ & $0.811 \pm 0.002$ & $0.822 \pm 0.002$ & $0.817 \pm 0.008$ \\
\hline \multirow[t]{2}{*}{$\Phi_{\mathrm{PSII}}$} & 22 & $0.613 \pm 0.012$ & $0.573 \pm 0.020$ & $0.675 \pm 0.006$ & $0.651 \pm 0.013$ \\
\hline & 16 & $0.452 \pm 0.016^{b}$ & $0.467 \pm 0.024^{\mathrm{a}}$ & $0.644 \pm 0.007^{\mathrm{a}}$ & $0.639 \pm 0.013$ \\
\hline \multirow[t]{2}{*}{$A_{N}$} & 22 & $10.9 \pm 0.2$ & $14.6 \pm 0.9$ & $13.1 \pm 0.2$ & $14.8 \pm 1.6$ \\
\hline & 16 & $3.4 \pm 0.4^{c}$ & $8.3 \pm 0.4^{c}$ & $8.5 \pm 0.2^{c}$ & $11.7 \pm 1.1$ \\
\hline \multirow[t]{2}{*}{$\mathrm{A}_{\text {sat }}$} & 22 & $15.5 \pm 0.4$ & $21.6 \pm 1.4$ & $28.9 \pm 2.1$ & $24.9 \pm 2.6$ \\
\hline & 16 & $7.0 \pm 0.3^{c}$ & $12.8 \pm 0.5^{c}$ & $23.6 \pm 1.4$ & $20.1 \pm 2.0$ \\
\hline
\end{tabular}

Data represent the mean $( \pm \mathrm{SE})$ of three to five individual plants. $\mathrm{F}_{\mathrm{v}} / \mathrm{F}_{\mathrm{m}}$ and $\Phi_{\mathrm{PSII}}$ are dimensionless, while the units of $\mathrm{A}_{\mathrm{N}}$ and $\mathrm{A}_{\text {sat }}$ are $\mu \mathrm{mol} \mathrm{m} \mathrm{m}^{-2} \mathrm{~s}^{-1}$. Significant difference is denoted as $\mathrm{a}, \mathrm{b}$, and $\mathrm{c}$ for $P<$ $0.05,<0.01$, and $<0.001$, respectively. ${ }^{*}(\mathrm{n})$ is number of weeks exposed to SOT. 
High amount of sucrose-6-phosphate was detected in leaves of the wild accession LA385

Five distinct, phosphorylated sugars were measured in the leaf samples, i.e. sucrose-6phosphate (Suc-6-P), glucose-1-phosphate (Glu-1-P), glucose-6-phosphate (Glu-6-P), mannose-6-phosphate (Man-6-P), and fructose-6-phosphate (Fru-6-P) (Figure 2). Strikingly Suc-6-P was abundant in LA385 leaves but was not detected to any significant level in Moneymaker leaves. Furthermore the content of Man-6-P and Fru-6-P was also higher in LA385 than in Moneymaker (Supplementary Table 2). In general, the phosphorylated sugars profile in leaves of both genotypes was not affected at SOT, except Glu-6-P content in week 16 , which was higher at $16^{\circ} \mathrm{C}$ compared to $22^{\circ} \mathrm{C}$. In addition, it appeared that there was no combined effect of temperature and genotype on the contents of these phosphorylated sugars (Supplementary Table 2).
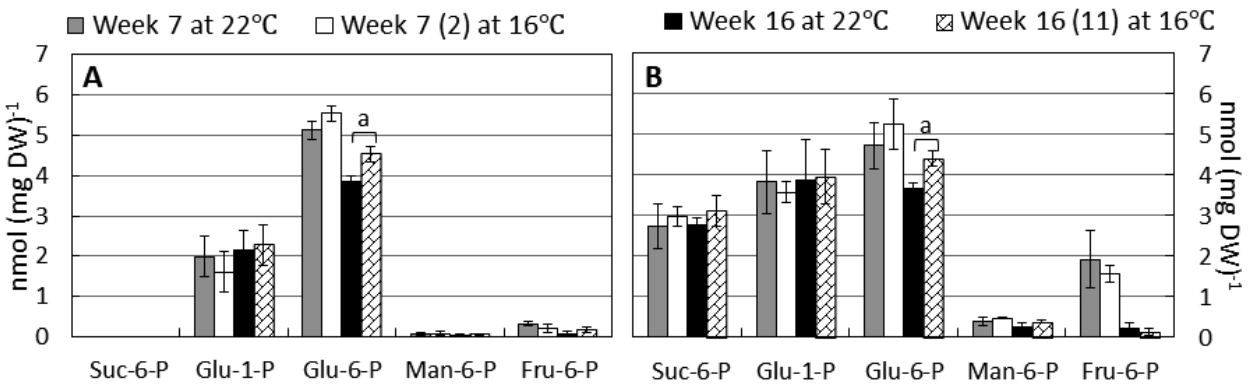

FIGURE 2 | Effect of SOT on several phosphorylated sugars in leaves at weeks 7 and 16. (Panel A and B) depicts sucrose-6-phosphate (Suc-6-P), glucose-1-phosphate (Gluc-1-P), glucose-6-phosphate (Glu-6-P), manose-6-phosphate (Man-6-6P), and fructose-6-phosphate (Fru-6-P) content of cv. Moneymaker and wild accession LA385, respectively. (n) is the number of weeks exposed to SOT. The error bars represent standard error of mean of fie plants. Significant difference is denoted as "a" for $\mathrm{P}<0.05$.

\section{Cultivar Moneymaker and wild accession LA385 had different carbohydrate profiles in fruits and leaves}

The neutral sugars and starch profiles in leaves of Moneymaker and LA385 (Figure 3) were different. Moneymaker leaves accumulated significantly more glucose, fructose, and sucrose at SOT than at CT in week 7, but this accumulation of neutral sugars stopped in week 16 (Figure 3A). Compared to Moneymaker, LA385 leaves displayed an opposite trend of neutral sugar accumulation: they contained fewer neutral sugars at SOT, especially fructose and glucose, but accumulated more sucrose (Figure 3B). As the LA385 plants became more mature, the sucrose content of their leaves increased more than twofold (cf week 7 leaves with week 16 leaves). This interactive effect of temperature and genotype on sucrose content in leaves was also shown in Supplementary Table 2. Another striking difference between Moneymaker and LA385 leaves was their starch content (Figure 3C). Compared to LA385, Moneymaker leaves had a high starch content of approximately $200 \mathrm{~g} \mathrm{mg} \mathrm{DW}^{-1}$ in leaves at both temperatures in week 7 , which decreased by about $50 \%$ at CT by week 16 while the starch content of the SOT leaves at week 16 similar to the levels found at week 7 . The starch 
content of the LA385 leaves increased both with age, being higher at week 16 than week 7 , and with decreased growth temperature, being higher at SOT than at CT. These effects of age and growth temperature for LA385 leaves were approximately additive, so the lowest starch levels were found in CT leaves at week 7 and the highest at SOT leaves at week 16. Though starch content in LA385 was higher at week 16 than week 7, it was at week 7 that fruits had begun to form and fruits are strong sinks. Overall the ratio of sucrose to starch content in Moneymaker leaves was approximately $4 \%$ and $7 \%$ at SOT and CT, respectively. Compared to Moneymaker leaves, the ratio of sucrose over starch content in LA385 leaves were about 10 -fold higher, i.e. $30 \%$ and $70 \%$ at SOT and CT, respectively (Figure 3D).

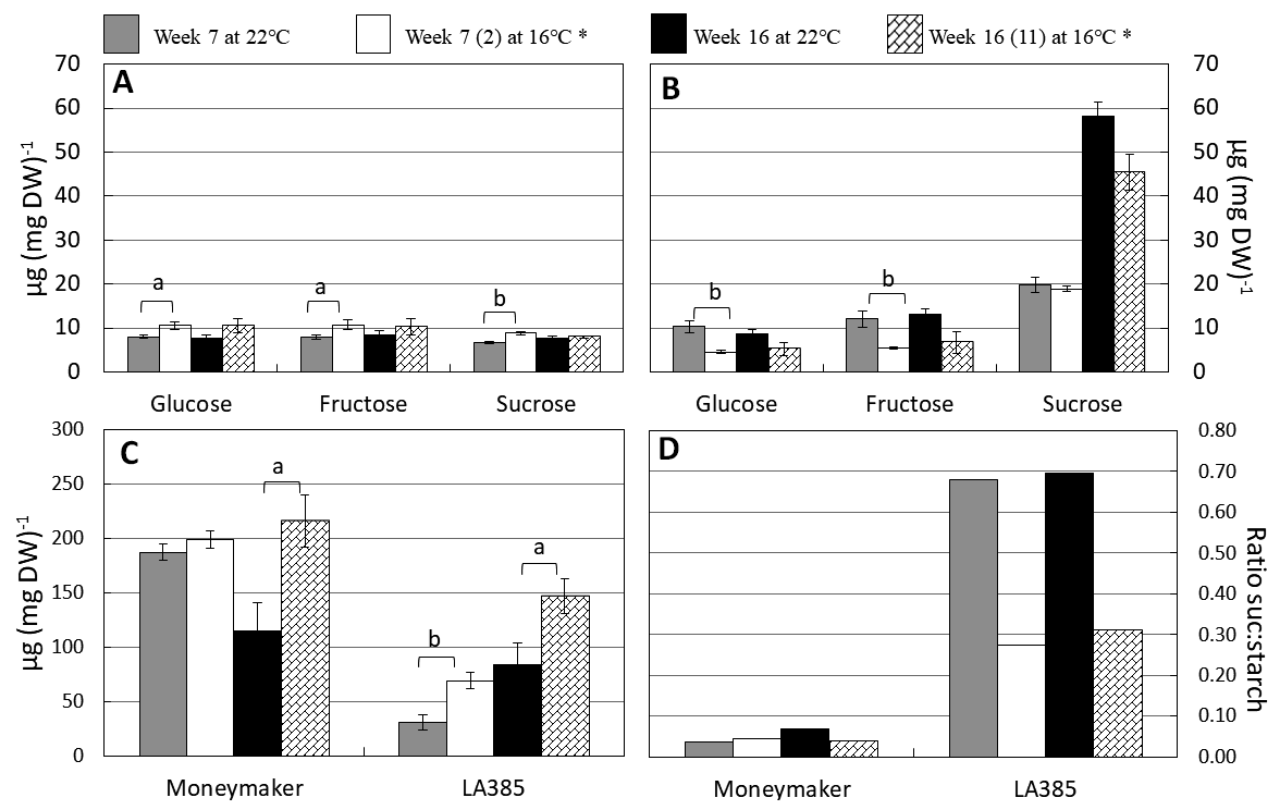

FIGURE 3 | Effect of SOT on neutral sugars, starch content, and sucrose: starch ratio in leaves. (Panels $\mathbf{A}$ and B) show the glucose, fructose, and sucrose contents at weeks 7 and 16 of cv. Moneymaker and wild accession LA385, respectively. (Panels $\mathbf{C}$ and D) show the starch content and sucrose: starch ratio, respectively. $*(\mathrm{n})$ is the number of weeks exposed to SOT. The error bars represent standard error of mean of fie plants. Significant difference is denoted as a, b, and c for $\mathrm{P}<$ $0.05,<0.01$, and $<0.001$, respectively. 
The profile of neutral sugars in fruits was distinct between two genotypes. Hexoses (i.e. glucose and fructose) and sucrose are the primary sugars of Moneymaker and LA385, respectively (Figure 4). Moneymaker immature green fruits contained 13-fold and 22-fold more hexoses compared to sucrose at CT and SOT respectively; while its ripe red fruits contained two-fold more hexoses and two-fold less sucrose compared to the green fruits at CT (Figure 4B). On the other hand the predominant neutral sugar in LA385 fruits was sucrose which was at least two-fold higher than hexoses (Figure 4A). The neutral sugars content of Moneymaker and LA385 fruits appeared to be affected differently at SOT. The content of glucose, fructose and sucrose in Moneymaker fruits was sharply decreased at SOT, whereas only the sucrose content of LA385 fruits was significantly reduced at SOT. This interactive effect of temperature and genotype on neutral sugars was also displayed in Supplementary Table 2, especially for fructose and sucrose.

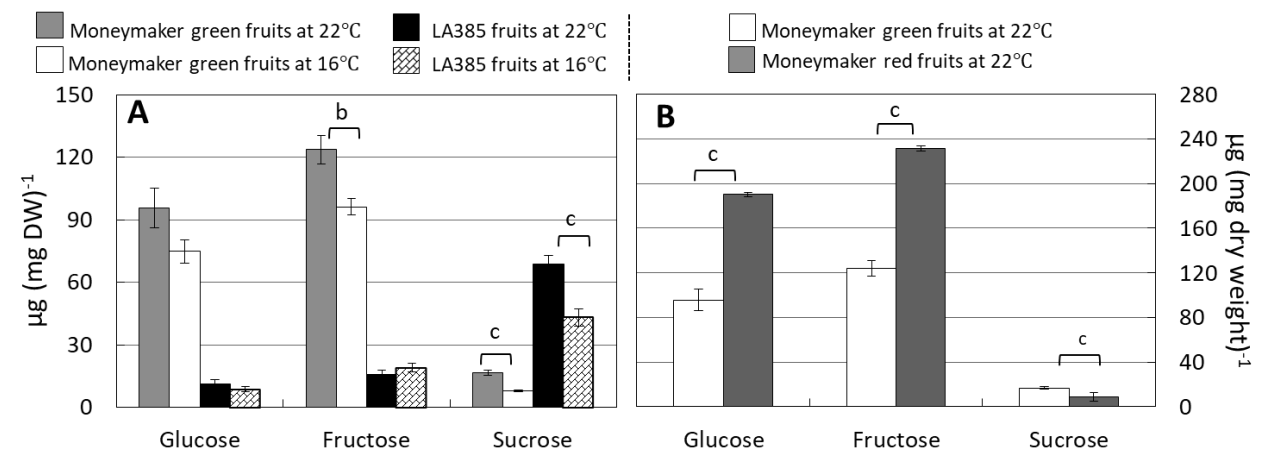

FIGURE 4 | Effect of SOT on neutral sugars in fruits of cv. Moneymaker (MM) and wild accession LA385 at week 16 (or 11 weeks exposed to SOT). (Panel A) displays the neutral sugar profile in green fruits of both tomato genotypes, while (Panel B) shows the content of neutral sugars in green and red fruits of cv. Moneymaker. The error bars represent standard error of mean of five different plants. Significant difference is denoted as $\mathrm{a}, \mathrm{b}$, and $\mathrm{c}$ for $\mathrm{P}<0.05,<0.01$, and $<0.001$, respectively. 


\section{Discussion}

\section{Wild accession LA385 has better acclimation from affected plant morphology at suboptimal temperature}

Suboptimal temperature (SOT) treatment resulted in a clear delay in growth and development in the S. lycopersicum cv Moneymaker, whereas the high altitude wild species S. arcanum LA385 was able to acclimate from the SOT-induced delay during the fruit development phase. The reduction in specific leaf area (SLA) at SOT in Moneymaker (Table 1) is in agreement with previous reports (Venema et al. 1999b, Atkin et al. 2006b, Kläring et al. 2015). Given that the leaf dry matter (DM) content in Moneymaker was unaffected by SOT (Supplementary Figure 1A,B), the reduction of SLA was likely offset by the increase in leaf thickness. According to Hoek et al. (1993) the larger size of the cells at SOT is the cause of the increase in leaf thickness, and these larger cells are able to store more starch (Venema et al. 1999a, Klopotek and Kläring 2014). In addition, the increase in leaf thickness and total protein concentration has been suggested to be connected to acclimation to low temperature (Pyl et al. 2012). In this study we observed that at early growth stage, the SLA of LA385 was reduced at SOT, which is in line with the findings of Venema et al. (1999b) (who reported only on young tomato plants). Furthermore we show, for the first time, that the SLA of LA385 at SOT was able to acclimate to close to the value at CT during the fruit development stage. It is possible that this acclimation of SLA at SOT was due to the slight reduction in leaf DM content of wild accession LA385 in week 16 (Supplementary Figure 1A). In turn, the slight decrease in leaf DM content of LA385 at SOT had a smaller effect on the DM allocation to other plant organs and helped maintain whole plant growth.

\section{Suboptimal temperature decreases dry matter yield and fruit growth in cv Moneymaker but not in the wild accession LA385}

Suboptimal temperature strongly affected the total dry matter (DM) production and DM allocation to fruits in cv Moneymaker, whereas it had a negligible influence on those parameters in the high altitude wild accession LA385 (Table 1, Table 2, and Supplementary Table 2). At both CT and SOT conditions, however, DM allocation to fruits in LA385 was always lower than DM allocation to fruits in Moneymaker. The drop in total DM yield of Moneymaker at SOT was paralleled by the sharp decrease in its DM allocation to fruits (Table 1, Table 2 and Figure 1). These reductions could be attributed to the profoundly lower accumulation of neutral sugars in the green fruits of Moneymaker at SOT in contrast to that found in ripe fruits at CT (Figure 4B). Furthermore, this study also observed the delay of first fruit-harvest in cultivated tomatoes due to lower temperatures, as reported by others (Hurd and Graves 1985, Kläring et al. 2015); there were no ripe fruits on Moneymaker at SOT in week 16, while by week 16 several ripe fruits had developed at CT (data not shown). Therefore it is likely that, compared to CT, a longer growing time at SOT would be required for fruit ripening in Moneymaker and for the accumulation of DM to the levels found at CT. This hypothesis is supported by previous research in which the tomato cultivars grown for at least four weeks longer at SOT than in this study showed no reduction in the total DM yield and DM fraction to fruits at SOT compared to CT (Heuvelink 1995, Kläring et al. 2015). We 
also show here, for the first time, the impact of low temperatures on the total DM yield and DM allocation of LA385 from flower onset until fruit development stage. The stability of total DM yield and DM fraction to sink fruits at SOT compared to CT demonstrates the cold tolerance of this wild accession and its potential value as a source of this trait. The cold tolerance of LA385 is likely due to its ability to acclimate its source-sink balance during fruit development stage, which could be seen in the pronounced increase in total leaf area and number of sink fruits and similar level of DM allocation to fruits of LA385 at SOT compared to CT (Table 1, Table 2, and Figure 1) - the ability of LA385 to continue to grow better at SOT than Moneymaker plays a role in this.

\section{Suboptimal temperature strongly affects photosynthetic capacity of cultivated tomato but less in wild accession}

Photosynthesis in LA385 shows much better adaptation to SOT than does that of Moneymaker. The maximum, dark-adapted quantum efficiency of photosystem II (PSII) photochemistry, which is estimated as the ratio of variable chlorophyll fluorescence yield and the maximum fluorescence yield $\left(\mathrm{F}_{\mathrm{v}} / \mathrm{F}_{\mathrm{m}}\right)$, is known to be a good parameter with which to measure the accumulation of stress in a plant. The $\mathrm{F}_{\mathrm{v}} / \mathrm{F}_{\mathrm{m}}$ of $\mathrm{C} 3$ plants under optimal growth condition is around 0.83 (when measured with red excitation of photosystem II) and a value below 0.80 is generally taken to indicate the advent of photo-inhibition (Björkman and Demmig 1987). All the $F_{v} / F_{m}$ of both plant genotypes in this study were around $0.81-0.82$ despite of the low temperatures to which some plants were exposed (Table 3). Therefore, the plants in this study did not experience a temperature stress to an extent necessary to produce damage to PSII. However, SOT had different effects on the $\mathrm{CO}_{2}$ assimilation rates of Moneymaker and LA385. In the case of Moneymaker at SOT we observed a significant reduction in both the leaf net $\mathrm{CO}_{2}$ assimilation rate $\left(\mathrm{A}_{\mathrm{N}}\right)$ at growth irradiance $\left(300 \mu \mathrm{mol} \mathrm{m} \mathrm{m}^{-2} \mathrm{~s}^{-}\right.$ ${ }^{1}$ ) and the light saturated assimilation rate $\left(\mathrm{A}_{\text {sat }}\right)$ (Table 3 and Supplementary Figure 2). This reduction in $\mathrm{A}_{\mathrm{N}}$ was paralleled by a decreased $\Phi_{\mathrm{PSII}}$ under these conditions at $\mathrm{SOT}$ (Table 3). It was clear that at SOT Moneymaker was not able to sustain its photosynthetic capacity at levels comparable to those found at CT. In contrast to Moneymaker, we report for the first time that the wild accession LA385 could acclimate well to SOT; its $\mathrm{CO}_{2}$ assimilation rates and $\Phi_{\text {PSII }}$ at SOT were similar to those found at CT in week 16, during the fruit development phase (Supplementary Table 2). Additionally, all photosynthetic properties measured, i.e. Fv/Fm, $\Phi_{\text {PSII }}, A_{N}$ and $A_{\text {sat }}$, were consistently higher in LA385 than in Moneymaker throughout development and at both temperatures (Table $\mathbf{3}$ and Supplementary Figure 2). These findings indicate that LA385 is a potential source for the improvement of photosynthetic capacity in cultivated tomatoes.

\section{Higher sucrose metabolism plays an important role in photosynthetic capacity}

Downstream of photosynthesis is carbohydrate metabolism, in which fixed carbon is converted to end products, such as sucrose and starch, that can be used for plant growth and storage (reviewed in Smith and Stitt 2007). With higher photosynthetic capacity than Moneymaker, leaves of LA385 also appeared to have differential regulation or limitation of sucrose biosynthesis than Moneymaker (Supplementary Table 2). This could be seen via the 
differences in suc-6-P, a precursor of sucrose, and sucrose contents in leaves of these two distinct tomato accessions: i. while suc-6-P was abundant in LA385, it could not be detected in Moneymaker (Figure 2), ii. LA385 leaves also had twice the sucrose content of Moneymaker leaves at week 7 and five times the sucrose content at week 16 (Figure 3A,B). In plants, synthesis of sucrose is governed by two enzymes: sucrose phosphate synthase (SPS) catalyses the synthesis of suc-6-P from fructose-6-phosphate (Fru-6-P) and UDPglucose (Stitt et al. 1988); and sucrose phosphate phosphatase (SPP) irreversibly hydrolyses suc-6-P into sucrose. Via isotope dilution experiments, SPS and SPP have been suggested to form a complex facilitating the process of sucrose synthesis from suc-6-P (Echeverria et al. 1997). Using techniques such as bioluminescence resonance energy transfer (BRET) and bimolecular fluorescence complementation (BiFC), Maloney et al. (2015) confirmed that SPS and SPP indeed interact and form complex in planta, and overexpression of this complex in Arabidopsis and poplar promotes plant growth. Therefore it is possible that LA385 has a more efficient SPS-SPP complex than Moneymaker, which needs to be further investigated. On the other hand, no clear connection between the accumulation of soluble sugars or phosphorylated sugars in plant leaves and the decrease in photosynthetic capacity could be established in this study. Additionally, the fruits of LA385 accumulated four- to five-fold as much sucrose as counterparts of Moneymaker (Figure 4), which is in agreement with other work that showed LA385 is a sucrose accumulator and cultivated tomatoes such as cv Moneymaker is a hexose accumulator (Kortstee et al. 2007). Based on the osmotic principle, the accumulation of disaccharides (i.e. sucrose) instead of monosaccharides (i.e. hexoses) in sink organs would diminish the water uptake, which would then result in sinks with higher soluble solid content but lesser mass. The effect of tomato fruit sucrose accumulation was investigated by Chetelat et al. (1995), in which the authors generated BC5F2 plants containing the gene controlling fruit sucrose accumulation by backcrossing a high sucrose accumulator wild relative $S$. chmielewskii LA1028 with a cultivated tomato background as a hexose accumulator. The authors showed that there was no difference in total fruit mass between the sucrose and hexose accumulators; but sucrose accumulating plants were associated with several fruit quality parameters such as higher soluble solid content, juice consistency, serum viscosity, predicted paste yield. However, those authors did not include photosynthetic capacity measurement in their work. Therefore, further study needs to be done to investigate the effect of high sucrose accumulation in fruits on photosynthetic capacity. In addition to sugar metabolism, starch metabolism is another key process downstream of photosynthesis responsible for formation, breakdown, and interconversion of carbohydrate in plants. Both genotypes in our study showed significant accumulation of starch in their leaves at SOT, except for Moneymaker in week 7 (Figure 3C). In those plants with significantly higher accumulation of starch in leaves at SOT, it appeared that their photosynthetic parameters, such as $\Phi_{\mathrm{PSII}}, \mathrm{A}_{\mathrm{N}}$ and $\mathrm{A}_{\text {sat }}$, were lower than those at CT (Figure 3C and Table 3). However Moneymaker plants at SOT in week 7 having similar leaf starch content as the counterparts at CT also had those photosynthetic parameters significantly reduced at SOT compared to CT. Therefore leaf starch content did not seem to associate with the photosynthetic capacity. Comparing two cultivars and two high altitude wild species tomato young plants grown at $25 / 20^{\circ} \mathrm{C}$ and $16 / 14^{\circ} \mathrm{C}$, Venema et al. (1999b) also observed a significantly higher starch accumulation in leaves of all four genotypes at $16 / 14^{\circ} \mathrm{C}$ than at 
$25 / 20^{\circ} \mathrm{C}$, but no major decline in their photosynthetic capacity. In another study, Goldschmidt and Huber (1992) showed a correlation in a reduction in maximum photosynthetic capacity $\left(\mathrm{A}_{\max }\right)$ and high leaf starch content among several plant species responded to girdling, yet also observed a high percentage of inhibition in $\mathrm{A}_{\max }$ in girded leaves of a starchless tobacco mutant. Similar findings were also found in the work of Li et al. (2018), in which those authors exposed two zoysiagrass genotypes, cold tolerant one native to cold climate area and cold sensitive one from warm climate are, to sub-optimal $18 / 12^{\circ} \mathrm{C}$, chilling $8 / 2^{\circ} \mathrm{C}$, and freezing $2 /-4^{\circ} \mathrm{C}$ temperatures compared to the control $30 / 25^{\circ} \mathrm{C}$. Those authors observed that the cold tolerant genotype had a slight higher $\mathrm{A}_{\mathrm{N}}$ and a significantly higher accumulation of sucrose, trehalose, fructan, and starch comparing to the cold sensitive genotype at lower temperatures. Together with these studies, the data of our study also suggests that the high accumulation of starch is not the main cause for reduction in photosynthesis capacity. Furthermore, there was a clear distinction in sucrose: starch ratio between the leaves of Moneymaker and LA385, in which this ratio in LA385 was 10 fold higher than in Moneymaker (Figure 3D). This finding implies that the distinct sucrose metabolism in both leaves and fruits of wild accession LA385 could be one of the keys to support its higher photosynthetic capacity compared to that in Moneymaker. We have shown in a recent study that the heterologously expressed protein sucrose synthase 3 (SUSY3) of LA385 could hydrolyze sucrose more efficiently compared to the counterpart of Moneymaker (Dinh et al. 2018). Additionally, SUSY has been shown to interact with multiprotein complex involved in cellulose/callose biosynthesis in main crops such as cotton, tobacco, and wood (Nakai et al. 1999, Persia et al. 2008, Coleman et al. 2009, Fujii et al. 2010). Interestingly, Ntatsi et al. (2017) reported an increase in expression of genes related to cellulose biosynthesis in the rootstock of cold tolerant tomato $S$. habrochaites LA1777 when grown at suboptimal root temperature of $15^{\circ} \mathrm{C}$, whereas in the same growth temperature those genes were not upregulated in the rootstock of cold sensitive tomato Moneymaker. Therefore, SUSY and other players involved in sucrose metabolism would be of interest for further investigation.

\section{Conclusion}

Growth at suboptimal temperatures leads to different effects on the tomato cultivar Moneymaker and the high altitude wild species $S$. arcanum LA385 with regards their growth, development, dry matter production and allocation, photosynthetic capacity, and carbohydrate metabolism from the reproductive phase to the later phases of fruit development. To our knowledge, this current work is the first report on the effect of SOT with a wild species during the reproductive stage, where plants are most sensitive to temperature stress, up to the later stage of fruit development. While cv Moneymaker is profoundly affected by SOT, the wild accession LA385 is shown to acclimate well to SOT at the stage of fruit development with high photosynthetic capacity accompanied by active sucrose metabolism. Among our findings, the highly active sucrose metabolism in wild accession LA385 is the most interesting lead that we will investigate further. Getting more insights about the sucrose metabolism in this wild accession at SOT might facilitate the breeding program for new cultivars performing well at SOT, which might allow reductions in 
energy use for heating and bring the tomato greenhouse cultivation in temperate region one step closer to sustainability.

\section{Author contributions}

Q-D.D. and L.M.T did the experimental planning; Q-D.D., A.D., H.F., and G.T. performed data acquisition and analysis; Q-D.D. drafted the manuscript; R.G.F.V., J.H., and L.M.T. reviewed the manuscript critically. L.M.T. coordinated the project's funding.

\section{Funding}

This work was carried out within the research programme of BioSolar Cells led by René Klein Lankhorst, co-financed by the Dutch Ministry of Economic Affairs.

\section{Acknowledgements}

We thank Anne Kortstee at Plant Breeding WUR for advising about the tomato accessions; Andre Maassen, Gerrit Stunnenberg, Taede Stoker, and Rohan van Genderen from Unifarm WUR for taking good care of the plants; Alejandro Bustamante, Elias Kaiser, and Wim van Ieperen from Horticultural Supply Chains WUR for technical support with LICOR-6400 usage; Christel Denneboom, Xuan Xu, Behzad Rashidi, and Ernest Aliche at Plant Breeding WUR for helping with harvesting and plant phenotyping. 


\section{Supplementary Material}

High altitude wild species Solanum arcanum LA385 - a potential source for improvement of plant growth and photosynthetic performance at suboptimal temperatures

Quy-Dung Dinh ${ }^{1,2}$, Annemarie Dechesne ${ }^{1}$, Heleen Furrer ${ }^{1}$, Graham Taylor ${ }^{3}$, Richard G. F. Visser $^{1}$, Jeremy Harbinson ${ }^{3}$, and Luisa M. Trindade ${ }^{1^{*}}$

* Correspondence: Luisa Trindade: luisa.trindade@wur.nl

Supplementary Table 1. Concentration of macro- and micro-nutrients inside the nutrient solution B with EC 2.1 and $\mathrm{pH} 5.5$

\begin{tabular}{lll}
\hline Nutrient & mmol I $^{-1}$ & micromole I $^{-1}$ \\
\hline $\mathrm{NH}_{4}$ & 1.2 & \\
$\mathrm{~K}$ & 7.2 & \\
$\mathrm{Ca}$ & 4.0 & \\
$\mathrm{Mg}$ & 1.82 & \\
$\mathrm{NO}_{3}$ & 12.4 & \\
$\mathrm{SO}$ & 3.3 & \\
$\mathrm{P}$ & 1.0 & \\
$\mathrm{Fe}$ & & 35.0 \\
$\mathrm{Mn}$ & & 8.0 \\
$\mathrm{Zn}$ & & 5.0 \\
$\mathrm{~B}$ & & 20.0 \\
$\mathrm{Cu}$ & & 0.5 \\
$\mathrm{Mo}$ & & 0.5 \\
\hline & & \\
\hline
\end{tabular}


Supplementary Table 2: $P$-values of a two-way ANOVA testing for the effects of temperature treatment $\left(22^{\circ} \mathrm{C}\right.$ vs $16^{\circ} \mathrm{C}$ ) and genotypes (cv Moneymaker vs wild species LA385) on all the studied parameters at week 7 ( 2 weeks at SOT) and week 16 (11 weeks at SOT)

\begin{tabular}{|c|c|c|c|c|}
\hline Parameters & Week & Temperature & Genotype & Temperature * Genotype \\
\hline \multirow[t]{2}{*}{ Total leaf area } & W7 (2) & $<0.05$ & $<0.001$ & 0.592 \\
\hline & W16 (11) & 0.084 & $<0.001$ & $<0.01$ \\
\hline \multirow[t]{2}{*}{ Specific leaf area } & W7 (2) & $<0.001$ & $<0.05$ & $<0.05$ \\
\hline & W16 (11) & $<0.001$ & $<0.05$ & $<0.01$ \\
\hline \multirow[t]{2}{*}{ Relative leaf thickness } & W7 (2) & $<0.05$ & 0.469 & 0.063 \\
\hline & W16 (11) & $<0.01$ & $<0.01$ & 0.308 \\
\hline \multirow[t]{2}{*}{ Total fresh weight } & W7 (2) & 0.175 & $<0.001$ & 0.683 \\
\hline & W16 (11) & $<0.001$ & 0.001 & $<0.001$ \\
\hline \multirow[t]{2}{*}{ Total dry weight } & W7 (2) & $<0.01$ & 0.001 & 0.981 \\
\hline & W16 (11) & $<0.001$ & $<0.001$ & $<0.00$ \\
\hline \multirow[t]{2}{*}{ Leaf dry matter content } & W7 (2) & $<0.01$ & 0.760 & $<0.05$ \\
\hline & W16 (11) & 0.354 & $<0.001$ & $<0.05$ \\
\hline \multirow[t]{2}{*}{ Leaf to root DM ratio } & W7 (2) & $<0.05$ & 0.001 & 0.254 \\
\hline & W16 (11) & $<0.001$ & $<0.001$ & 0.816 \\
\hline \multirow[t]{2}{*}{ Number of fruits } & W7 (2) & $\mathrm{n} / \mathrm{a}$ & $\mathrm{n} / \mathrm{a}$ & $\mathrm{n} / \mathrm{a}$ \\
\hline & W16 (11) & 0.343 & 0.644 & $<0.01$ \\
\hline \multirow[t]{2}{*}{ Leaf DM fraction } & W7 (2) & $<0.01$ & $<0.001$ & 0.762 \\
\hline & W16 (11) & $<0.001$ & $<0.001$ & 0.055 \\
\hline \multirow[t]{2}{*}{ Stem DM fraction } & W7 (2) & $<0.05$ & $<0.001$ & 0.820 \\
\hline & W16 (11) & 0.570 & $<0.001$ & $<0.01$ \\
\hline \multirow[t]{2}{*}{ Root DM fraction } & W7 (2) & 0.085 & $<0.001$ & 0.889 \\
\hline & W16 (11) & 0.663 & $<0.001$ & 0.140 \\
\hline \multirow[t]{2}{*}{ Fruit DM fraction } & W7 (2) & $\mathrm{n} / \mathrm{a}$ & $\mathrm{n} / \mathrm{a}$ & $\mathrm{n} / \mathrm{a}$ \\
\hline & W16 (11) & $<0.001$ & $<0.001$ & $<0.001$ \\
\hline
\end{tabular}


Supplementary Table 2 (cont.): $P$-values of a two-way ANOVA testing for the effects of temperature treatment $\left(22^{\circ} \mathrm{C}\right.$ vs $\left.16^{\circ} \mathrm{C}\right)$ and genotypes (cv Moneymaker vs wild species LA385) on all the studied parameters at week 7 ( 2 weeks at SOT) and week 16 (11 weeks at SOT)

\begin{tabular}{|c|c|c|c|c|}
\hline Parameters & Week & Temperature & Genotype & $\begin{array}{l}\text { Temperature * } \\
\text { Genotype }\end{array}$ \\
\hline \multirow[t]{2}{*}{ Sucrose-6-phosphate in leaf } & W7 (2) & 0.709 & $<0.001$ & 0.709 \\
\hline & W16 (11) & 0.447 & 0.001 & 0.447 \\
\hline \multirow[t]{2}{*}{ Glucose-1-phosphate in leaf } & W7 (2) & 0.564 & $<0.01$ & 0.902 \\
\hline & W16 (11) & 0.862 & $<0.05$ & 0.973 \\
\hline \multirow[t]{2}{*}{ Glucose-6-phosphate in leaf } & W7 (2) & 0.307 & 0.434 & 0.917 \\
\hline & W16 (11) & $<0.001$ & 0.303 & 0.899 \\
\hline \multirow[t]{2}{*}{ Mannose-6-phosphate in leaf } & W7 (2) & 0.357 & $<0.001$ & 0.613 \\
\hline & W16 (11) & 0.158 & $<0.001$ & 0.358 \\
\hline \multirow[t]{2}{*}{ Fructose-6-phosphate in leaf } & W7 (2) & 0.547 & $<0.01$ & 0.747 \\
\hline & W16 (11) & 0.961 & 0.620 & 0.412 \\
\hline \multirow[t]{2}{*}{ Glucose in leaf } & W7 (2) & 0.126 & $<0.05$ & $<0.001$ \\
\hline & W16 (11) & 0.994 & 0.081 & $<0.05$ \\
\hline \multirow[t]{2}{*}{ Fructose in leaf } & W7 (2) & 0.452 & 0.283 & $<0.05$ \\
\hline & W16 (11) & 0.248 & 0.870 & $<0.05$ \\
\hline \multirow[t]{2}{*}{ Sucrose in leaf } & W7 (2) & 0.855 & $<0.001$ & $<0.05$ \\
\hline & W16 (11) & $<0.05$ & 0.001 & $<0.05$ \\
\hline \multirow[t]{2}{*}{ Starch in leaf } & W7 (2) & $<0.01$ & $<0.001$ & 0.098 \\
\hline & W16 (11) & $<0.01$ & $<0.05$ & 0.293 \\
\hline \multirow[t]{2}{*}{ Glucose in (green) fruit } & W7 (2) & $\mathrm{n} / \mathrm{a}$ & $\mathrm{n} / \mathrm{a}$ & $\mathrm{n} / \mathrm{a}$ \\
\hline & W16 (11) & 0.054 & $<0.001$ & 0.128 \\
\hline \multirow[t]{2}{*}{ Fructose in (green) fruit } & W7 (2) & $\mathrm{n} / \mathrm{a}$ & $\mathrm{n} / \mathrm{a}$ & $\mathrm{n} / \mathrm{a}$ \\
\hline & W16 (11) & $<0.05$ & $<0.001$ & $<0.01$ \\
\hline \multirow[t]{2}{*}{ Sucrose in (green) fruit } & W7 (2) & $\mathrm{n} / \mathrm{a}$ & $\mathrm{n} / \mathrm{a}$ & $\mathrm{n} / \mathrm{a}$ \\
\hline & W16 (11) & $<0.001$ & $<0.001$ & $<0.05$ \\
\hline \multirow[t]{2}{*}{$\mathrm{Fv} / \mathrm{Fm}$} & W7 (2) & 0.105 & $<0.001$ & 0.187 \\
\hline & W16 (11) & 0.106 & $<0.05$ & 0.780 \\
\hline \multirow[t]{2}{*}{$\Phi_{\text {PSII }}$ at PFD300 } & W7 (2) & $<0.001$ & $<0.001$ & $<0.001$ \\
\hline & W16 (11) & $<0.01$ & $<0.001$ & $<0.05$ \\
\hline \multirow[t]{2}{*}{$\mathrm{A}_{\mathrm{N}}$ at PDF300 } & W7 (2) & $<0.001$ & $<0.001$ & $<0.01$ \\
\hline & W16 (11) & $<0.01$ & 0.131 & 0.185 \\
\hline \multirow[t]{2}{*}{$\mathrm{A}_{\text {sat }}$} & W7 (2) & $<0.001$ & $<0.001$ & 0.230 \\
\hline & W16 (11) & $<0.001$ & $<0.05$ & 0.135 \\
\hline
\end{tabular}




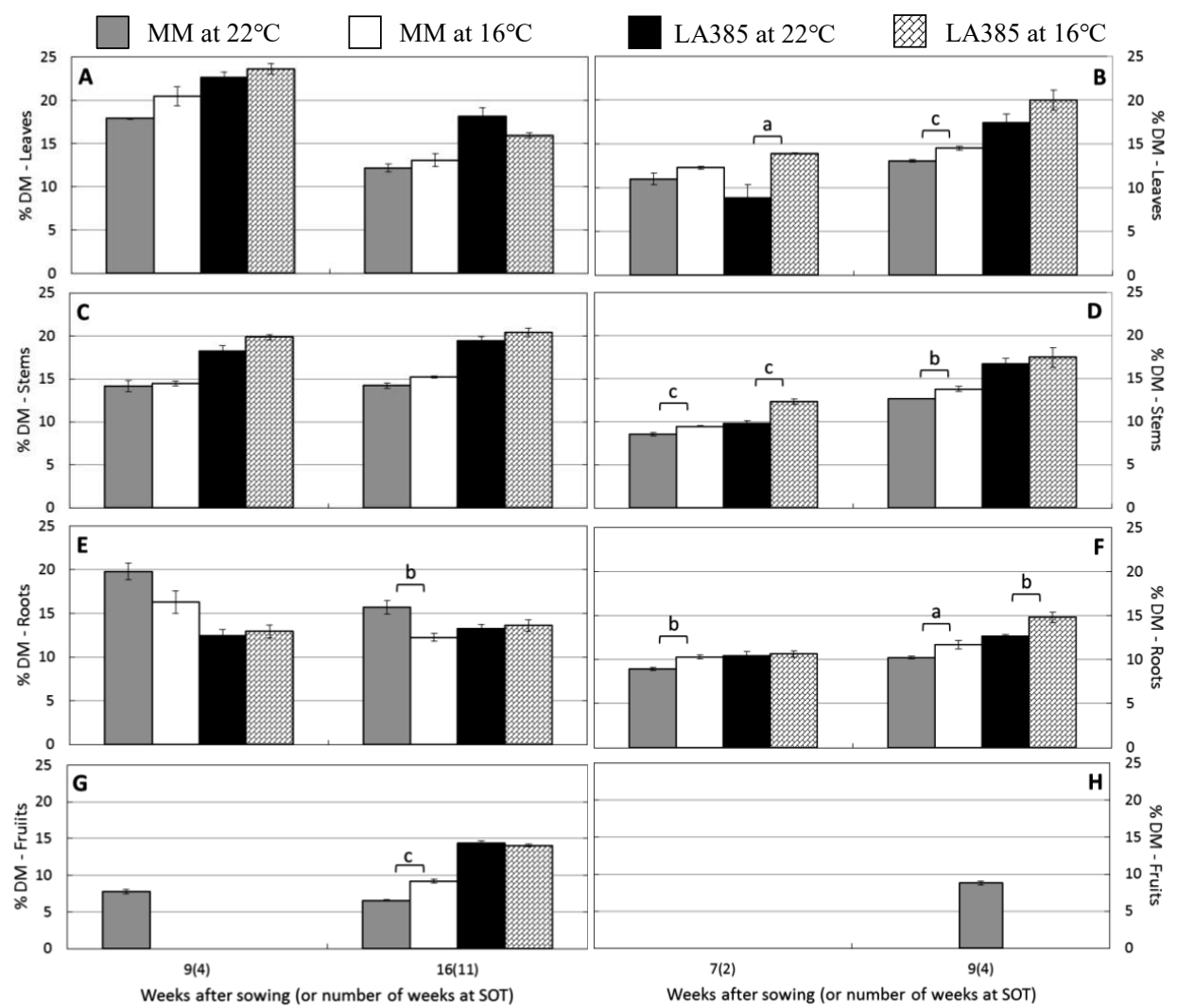

Supplementary Figure 1. Effect of SOT on dry matter content of different tomato organs of cv Moneymaker (MM) and wild species S. arcanum LA385. Panels on the left and right are from trial A and trial B, respectively. (n) is the number of weeks exposed to SOT. The error bars represent standard error of five individual plants $( \pm$ SE), except week 9 of trial B with three plants. Significant difference is denoted as a, $\mathrm{b}$, and $\mathrm{c}$ for $P<0.05,<0.01$, and $<0.001$, respectively. 


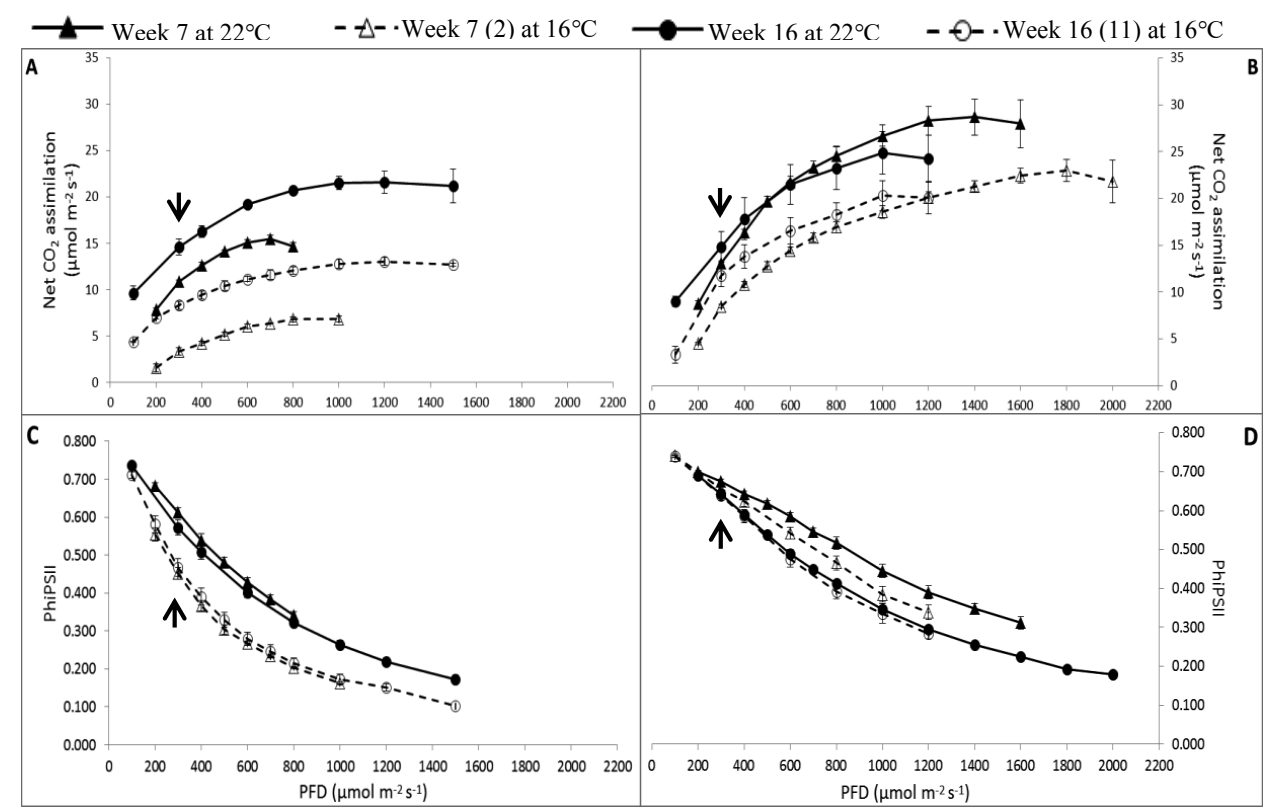

Supplementary Figure 2. Effect of SOT on photosynthetic parameters in week 7 (2) and week 16 (11) of cv Moneymaker and wild species $S$. arcanum LA385. Net $\mathrm{CO}_{2}$ assimilation rate $\left(\mathrm{A}_{\mathrm{N}}\right)$ and PhiPSII as a function of increasing light intensity starting from 0 till $2000 \mu \mathrm{mol} \mathrm{m} \mathrm{m}^{-2} \mathrm{~s}^{-1}$ at $\mathrm{CO}_{2}$ concentration of $400 \mu \mathrm{mol} \mathrm{m} \mathrm{m}^{-2} \mathrm{~s}^{-1}$ are demonstrated in panel A-B and panel C-D, respectively. We stopped measuring $A_{N}$ with higher light intensity as soon as there was no more increase in $A_{N}$ compared to the previous light intensity. $*(n)$ is the number of weeks exposed to SOT. The arrows indicate the values at PFD of $300 \mu \mathrm{mol} \mathrm{m} \mathrm{s}^{-1}$. Data represent mean $( \pm \mathrm{SE})$ of three to four plants.

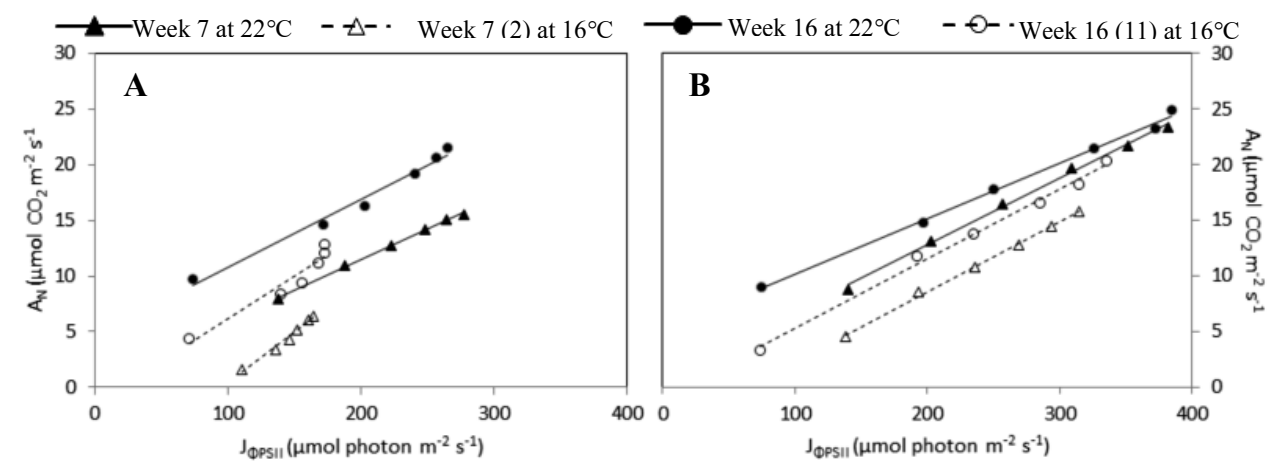

Supplementary Figure 3. Correlation between $\Phi_{\mathrm{PSII}}$ and net $\mathrm{CO}_{2}$ assimilation rate. The correlation is shown as function of irradiance $* \Phi_{\mathrm{PSII}}$ against $\mathrm{CO}_{2}$ fixation rate. The panel $\mathrm{A}$ and $\mathrm{B}$ show data of $\mathrm{cv}$. Moneymaker and wild accession LA385, respectively. The data were derived from the calculation with irradiance from $200-1000 \mu \mathrm{mol}$ photons $\mathrm{m}^{-2} \mathrm{~s}^{-1}$. The data represent mean of three to five independent measurements. 



\section{Chapter 3}

\section{Exploring natural genetic variation in tomato sucrose synthases on the basis of increased kinetic properties}

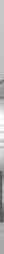

whin and

This chapter has been published as:

Quy-Dung Dinh, Richard Finkers, Adrie H. Westphal, Walter M. A. M. van Dongen, Richard G.F. Visser, Luisa M. Trindade (2018) Exploring natural genetic variation in tomato sucrose synthases on the basis of inçreased kinetic properties. PLoS ONE 13(10): e0206636. doi:10.1371/journal.pone.0206636 


\begin{abstract}
Sucrose synthase (SuSy) is one key enzyme directly hydrolyzing sucrose to supply substrates for plant metabolism, and is considered to be a biomarker for plant sink strength. Improvement in plant sink strength could lead to enhanced plant growth and yield. Cultivated tomatoes are known to have a narrow genetic diversity, which hampers further breeding for novel and improved traits in new cultivars. In this study, we observed limited genetic variation in SuSyl, SuSy3 and SuSy4 in 53 accessions of cultivated tomato and landraces, but identified a wealth of genetic diversity in 32 accessions of related wild species. The variation in the deduced amino acid sequences was grouped into 23, 22, and 17 distinct haplotypes for SuSy1/3/4, respectively. Strikingly, all known substrate binding sites were highly conserved, as well as most of the phosphorylation sites except in SuSy1. Two SuSy1 and three SuSy3 protein variants were heterologously expressed to study the effect of the amino acid changes on enzyme kinetic properties, i.e. maximal sucrose hydrolyzing capacity $\left(\mathrm{V}_{\max }\right)$, affinity for sucrose $\left(\mathrm{K}_{\mathrm{m}}\right)$, and catalytic efficiency $\left(\mathrm{V}_{\max } / \mathrm{K}_{\mathrm{m}}\right)$ at $25^{\circ} \mathrm{C}$ and $16^{\circ} \mathrm{C}$. SuSyl-haplotype\#3 containing phosphorylation site Ser-16 did not have an improvement in the kinetic properties compared to the reference SuSy1-haplotype\#1 containing Arg-16. Meanwhile SuSy3haplotype\#9 from a wild accession, containing four amino acid changes S53A, S106I, E727D and $\mathrm{K} 741 \mathrm{E}$, showed an increase in $\mathrm{Vmax} / \mathrm{Km}$ at $16^{\circ} \mathrm{C}$ compared to the reference SuSy3haplotype\#1. This study demonstrates that SuSy kinetic properties can be enhanced by exploiting natural variation, and the potential of this enzyme to improve sucrose metabolism and eventually sink strength in planta.
\end{abstract}




\section{Introduction}

In the plant kingdom, sucrose is the major product of photosynthesis. It is transported from photosynthetic source tissues to sink tissues as a main energy hub for metabolism, carbon source for synthesis of biomolecules, as well as an important signaling molecule (Koch 2004, Ruan 2014). Therefore, the mechanism by which sucrose metabolism is regulated has direct consequences on sink strength which is defined as the competitive ability of the organ to attract the photoassimilates (Marcelis 1996). As a result, sink strength plays an important role in plant growth, development, and biomass production. In plants sucrose synthase (SuSy) is, next to invertase, a class of enzymes directly involved in hydrolysis of sucrose in sink tissues. SuSy is a member of the glycosyltransferase family, which catalyzes a reversible hydrolysis of sucrose into UDP-glucose and fructose in the presence of UDP. Up till now, six SuSy genes have been described in tomato (shown in S2 File). Three genes SuSyl (Solyc12g009300, also known as TOMSSF or SUS2), SuSy3 (Solyc07g042550) and SuSy4 (Solyc09g098590) were cloned before the release of tomato genome sequence (Wang et al. 1993b, Chengappa 1998, Goren et al. 2011), and SuSy5 (Solyc07g042520), SuSy6 (Solyc03g098290) and SuSy7 (Solyc02g081300) were identified after that (Qin et al. 2016). These genes are shown to be expressed differentially in tomato plant tissues (Wang et al. 1993a, Wang et al. 1993b, Chengappa 1998, Kortstee et al. 2007, Goren et al. 2011, Qin et al. 2016, Goren et al. 2017). SuSyl is highly expressed in young fruits, whereas the expression level of $\mathrm{SuSy} 3$ is highest in mature fruits. The transcript level ofSuSy4 is most abundant in sink organs such as fruits, roots, and developing leaves, but about 200-fold lower than that ofSuSy3. Meanwhile Susy5 is highly expressed in stems and roots, the transcript level of Susy6 and $S u S y 7$ are very low in all the main tissues. At the protein level, SuSyl and SuSy3 isoforms share up to $91 \%$ of sequence identity, while they are only $68 \%$ and $73 \%$ identical to SuSy4 and SuSy5 respectively. SuSy6 and SuSy7 isoforms are up to $73 \%$ in sequence homology, but they are only $54-56 \%$ identical to the other isoforms. These SuSy1/3/4 isoforms have been reported to be present in cytoplasm, cell membrane, and mitochondria (Hardin et al. 2003, Anguenot et al. 2006, Barrero-Sicilia et al. 2011), but little is known about the compartmentation of SuSy5/6/7. So far, SuSy1 of Arabidopsis thaliana is the only plant SuSy with a resolved crystal structure, which showed that this enzyme is organized in tetramers (Zheng et al. 2011). The SuSy protein structure basically contains three distinct features: i. an Nterminal domain involved in cellular compartmentation which is sub-divided into a cellular targeting domain (CTD, length 116 amino acids) and an ENOD40 peptide-binding domain (EPDB, length 120 amino acids); ii. a GT-B glycosyltransferase domain (GT-B, length 548 amino acids); and iii. a C-terminal extension of 30 up to 40 amino acids. Several residues involved in substrate binding have been identified by X-ray diffraction of enzyme crystals in complex with: i) UDP-glucose, and ii) UDP and fructose. Even though the complex of AtSuSyl and sucrose is still missing, the residues involved in sucrose binding could be derived from the interaction with glucose and fructose of those two complexes. An important role of SuSy on plant sink strength has been reported in tomato, where tomato plants with reduced SuSy activity, obtained by down regulating the expression of the SuSyl allele by an antisense approach, were shown to have a reduced fruit setting and sucrose unloading capacity in their young fruits (D'Aoust et al. 1999). In addition, SuSy was 
also shown to play an essential role in cold tolerance in chickpeas, in which significantly higher activity of SuSy was observed in seeds of the cold tolerant lines compared to that of cold susceptible ones (Kaur et al. 2009). This cold tolerance trait could be an advantage for the tomato cultivars grown in heated glasshouses during winter because it would help to reduce energy consumption under such cultivation conditions. The sub-optimal temperature that we aim for is $16^{\circ} \mathrm{C}$, which is below the current optimum temperature $19-20^{\circ} \mathrm{C}$ for heated glasshouse tomato cultivation in the Netherlands, and well above the non-freezing temperature of $12^{\circ} \mathrm{C}$ which is known to cause physiological injury to tomato plants and their fruits when exposed for several weeks (Brüggemann et al. 1992, Heuvelink and Buiskool 1995). Therefore, enhancing tomato SuSy activity is of interest to increase sink strength in order to have a positive effect on plant growth, development, and yield at optimal as well as sub-optimal temperatures. For that reason, this study focused on exploring the natural genetic variation among the three SuSy1/3/4 isoforms using the available genome sequences of 84 accessions including cultivars, landraces, and wild species from The 150 Tomato Genome ReSequencing project (Aflitos et al. 2014), and one extra high-altitude wild species accession obtained from Tomato Genetics Resource Center in Davis, California (TGRC, http://tgrc.ucdavis.edu/). An in silico analysis was carried out to predict the potential effect of amino acid changes caused by natural variation on the modelled protein structure and functionality. Furthermore, three chosen variants of SuSy1 and SuSy3 from wild accessions along with the counterparts from cultivar Moneymaker as the reference control were cloned and expressed in a heterologous system to investigate their enzyme kinetics at control $\left(25^{\circ} \mathrm{C}\right)$ and low $\left(16^{\circ} \mathrm{C}\right)$ temperatures. 


\section{Materials and methods}

\section{Computational analysis of sucrose synthase sequences from 85 tomato accessions}

The genomic sequence $S u S y 1 / 3 / 4$ of 84 different tomato accessions, were obtained from The 150 Tomato Genome ReSequencing project, in which the raw data were submitted to the European Nucleotide Archive under project number PRJEB5235 (http://www.ebi.ac.uk/ena/data/view/PRJEB5235). Among these accessions 53 are cultivated and landrace accessions, whereas 31 are wild species accessions. In order to identify the possible coding sequences (cds) for SuSy proteins in the 84 accessions, the cds of SuSyl (GenBank: L19762), SuSy3 (GenBank: AJ011319), and SuSy4 (GenBank: HM180943) were aligned to these genomic sequences using the ClustalOmega program (http://www.ebi.ac.uk/Tools/msa/clustalo/). In addition, the cds of SuSyl/3/4 from the extra wild species accession Solanum arcanum LA385 were also included in this study. The obtained cds of 85 accessions were subsequently translated into amino acid sequences using BioEdit software v.6.0.6 (Tom Hall, Ibis Therapeutics, Carlsbad, USA).

We investigated in silico the effects of the amino acid substitutions within each protein sequence compared to the corresponding positions of the reference cultivar Heinz. Those studied effects included: 1) the potential impact of an amino acid change on protein function using the 'Protein Variation Effect Analyzer' program (PROVEAN) (Choi et al. 2012); 2) the possible influence of an amino acid change on the protein secondary and tertiary structure using the 'Protein Homology/analogY Recognition Engine V 2.0' program (Phyre2) (Kelley et al. 2015) and Modeller program v9.19 (Šali and Blundell 1993). Phyre2 also provided the 3D-models of the SUSY variants based on the available crystal structure of SuSyl of Arabidopsis thaliana (PDB entry 3S27), which were visualized with PyMol software v1.6 (The PyMOL Molecular Graphics System v1.6, Schrödinger, LLC, USA).

\section{Plants, bacterial and yeast strains, and plasmids used}

Accessions that were used to extract DNA for sequencing and cloning of specific SuSy genes were: S. lycopersicum cv Moneymaker (accession number LA2706), cv Large Red Cherry (accession number TR00018), S. neorickii G1.1601 (accession number CGN24193) and S. arcanum LA385 obtained from Tomato Genetics Resource Center in Davis, California (TGRC, http://tgrc.ucdavis.edu/). The cv Moneymaker was used in this study as the reference in place of cv Heinz because they both have the same SuSy1/3/4 protein sequences. Plasmid pGEM-T Easy (Promega, USA), PCR II-Blunt-TOPO (ThermoFisher Scientific, USA) were used to construct intermediate clones, while plasmid pDR195 (a gift from Dr. Wolf Frommer - Carnegie Institution for Science, USA) was used to make the expression clones. Competent cells of Escherichia coli strains DH5a and XL10-Gold were purchased from Invitrogen (USA) and Agilent (USA) respectively, whereas yeast Saccharomyces cerevisiae strain 22574d was provided by courtesy of Dr. Bruno André - Université Libre de Bruxelles, Belgium. 


\section{Cloning and expression of SuSy genes}

RNA was prepared from about $5 \mathrm{mg}$ fruit tissue ground in liquid nitrogen using a CTAB mini-isolation protocol (Untergasser 2008). The isolated RNA was then treated with DNase I (Invitrogen, USA), and converted into cDNA with iScript cDNA synthesis kit (BioRad, USA). Amplification of cDNA was achieved following a standard PCR protocol. The primer pairs for specific gene amplification can be found in S1 Table. All the amplifications for cloning steps were carried out with high fidelity PfuUltra Fusion II HS DNA polymerase (Agilent, USA). The amplified cDNA of $S u S y 1 / 3 / 4$ from cv. Moneymaker, and SuSyl/3/4 from wild accession LA385 were ligated with plasmid pGEM-T Easy, whereas SuSyl from LRC and Susy3 from wild accession G1.1601 were ligated with PCR II-Blunt-Topo plasmid. These ligation mixtures were transformed into competent $E$. coli $\mathrm{DH} 5 \alpha$ and positive clones were confirmed by sequencing. Subsequently the cDNA of these genes were amplified with primer pairs containing BamHI sites from the isolated plasmids, which resulted in fragment BamHI-SuSy-BamHI. The newly obtained fragment BamHI-SuSy-BamHI was digested with BamHI enzyme, ligated into the BamHI digested and dephosphorylated plasmid pDR195, and transformed into ultra-competent E. coli XL10-Gold (Agilent, USA). Positive clones were confirmed by restriction digestion and sequencing. The final expression plasmids were introduced into yeast $S$. cerevisiae strain $22574 \mathrm{~d}$ as described in the manual of the DUALmembrane starter kit (Dualsystems Biotech AG, Switzerland). Positive clones were selected on synthetic defined (SD) medium agar without uracil (Clontech, USA).

\section{Cultivation of yeast}

Cultivation of the transformed yeast harboring recombinant SUSY enzyme was done according to Römer et al. (2004) with some modifications. Briefly, a positive colony from a $\mathrm{SD}$ agar plate was cultivated in a $100 \mathrm{~mL}$ flask containing $12 \mathrm{~mL}$ SD medium with $2 \%$ glucose without uracil at $30^{\circ} \mathrm{C}$ overnight in an incubator shaker with an orbital speed of 200 $\mathrm{rpm}$. Subsequently the overnight culture was transferred into a $4 \mathrm{~L}$ flask containing $2 \mathrm{~L}$ of the same medium for $24 \mathrm{~h}$ incubation with an orbital speed of $130 \mathrm{rpm}$. The cells were then harvested by centrifugation at $4^{\circ} \mathrm{C}$ and $10,000 \mathrm{x}$ g and stored at $-20^{\circ} \mathrm{C}$ till further use.

\section{Enzyme purification}

An aliquot of $10 \mathrm{~g}$ harvested yeast cells were resuspended to $40 \%\left(\mathrm{w} \mathrm{v}^{-1}\right)$ in lysis buffer of 50 $\mathrm{mM}$ HEPES $\mathrm{pH}$ 7.5, $1 \mathrm{mM}$ dithiothreitol (DTT), $1 \mathrm{mM} \mathrm{Na}_{2}$ EDTA, $1 \mathrm{mM}$ phenylmethanesulfonyl fluoride (PMSF), 10\% glycerol, 1 M Leupeptin, and 1 M Pepstatin A. The resuspended cells were then lysed via 3 passages of 10,000 psi using a SLM-Aminco French Pressure Cell apparatus. The cell extract was subsequently cleared by centrifugation at $18,000 \times \mathrm{g}$ for $20 \mathrm{~min}$ at $4^{\circ} \mathrm{C}$. Afterward, the extract was purified with ion-exchange chromatography (IEX) and immobilized metal ion affinity chromatography (IMAC) according to Römer et al. (2004), using HiLoad Q Sepharose high performance and HiTrap IMAC Fast Flow columns (GE Life Sciences, USA) respectively. The purification of SuSy3haplotype\#1 (cv Moneymaker) and SuSy3-haplotype\#9 (wild accession LA385) were prepared by both IEX and IMAC, whereas SuSy3-haplotype\#10 (wild accession G1.1601), 
SuSy1-haplotype\#1 (cv Moneymaker), SuSy1-haplotype\#3 (cv Large Red Cherry), SuSy4haplotype\#1 (cv Moneymaker), and SuSy4-haplotype\#4 (wild accession LA385) were only purified with IEX. The protein concentration was measured with Pierce BCA Protein Assay Kit (ThermoFisher - Scientific, USA). Subsequently, the size of the purified products was estimated by SDS-PAGE and Native PAGE by using the Mini-PROTEAN TGX gel $4-20 \%$ (BioRad, USA). The expected product of $93 \mathrm{kDa}$ in SDS PAGE was not found in the purified product of both SuSy4-haplotype\#1 (cv Moneymaker) and SuSy4-haplotype\#4 (wild accession LA385) with IEX (data not shown), therefore they were excluded from the enzyme kinetics analysis.

\section{Enzyme kinetics analysis}

In this study we focused on the kinetics of the sucrose cleavage action of the enzymes under investigation. For enzyme kinetics assays, we used the highest purified enzymes that were prepared as described in section enzyme purification above, i.e. IMAC-purified SuSy3haplotype\#1 and haplotype\#9, IEX-purified SuSy3-haplotype\#10, SuSy1-haplotype\#1, and SuSy1-haplotype\#3. The kinetic constants $\mathrm{V}_{\max }$ and $\mathrm{K}_{\mathrm{m}}$ for sucrose were determined in a reaction mixture containing 20mM HEPES pH 7.5, $4 \mathrm{mM}$ UDP, and sucrose ranging from 10 to $500 \mathrm{mM}$. The reaction was started by adding $0.5-1.0 \mathrm{~g}$ purified SuSy enzyme into the reaction mixture and incubated at $25^{\circ} \mathrm{C}$ or $16^{\circ} \mathrm{C}$ for $0,5,10$, and $15 \mathrm{~min}$. The reaction was terminated by incubation at $95^{\circ} \mathrm{C}$ for $4 \mathrm{~min}$. The formation of UDP-glucose was measured in an assay containing $0.4 \mathrm{M}$ glycine $\mathrm{pH} 8.9,10 \mathrm{mM} \mathrm{MgCl}_{2}, 4 \mathrm{mM} \mathrm{NAD}^{+}$, and $0.02 \mathrm{U}$ of UDPglucose dehydrogenase. The amount of NADH formed in this reaction equals the amount of UDP-glucose formed in the reaction mixtures with SuSy enzymes. For the blanks, the reaction was without UDP addition. The amounts of UDP-glucose formed in $0,5,10$, and 15 min, corrected for the blank reactions, were plotted and fitted using a linear regression model. The slope of this line equals the initial rate of sucrose hydrolysis (v). Subsequently, the kinetic constants $\mathrm{V}_{\max }$ and $\mathrm{K}_{\mathrm{m}}$ were calculated by fitting the Michaelis-Menten curve. We define 1 unit $(U)$ of SuSy enzyme activity as the amount of enzyme that catalyzes the production of $1 \mathrm{~mol}$ of UDP-glucose per minute. All the chemicals and UDP-glucose dehydrogenase enzyme used for kinetics analysis were purchased from Sigma-Aldrich (USA). 


\section{Results}

\section{More variation in the coding sequences of SuSy of wild species than in cultivars and landraces}

The genomic sequences of SuSy1/3/4 of 85 accessions were aligned against known coding sequences of SuSyl (GenBank: L19762), SuSy3 (GenBank: AJ011319), and SuSy4 (GenBank: HM180943) to search for single nucleotide polymorphisms (SNPs). We found SNPs in both coding and non-coding regions with both synonymous and non-synonymous SNPs (data not shown). For the non-synonymous SNPs that lead to amino acid substitutions, the corresponding protein sequences were deduced and grouped into 23 distinct SuSyl sequences, 22 SuSy3 sequences, and 17 SuSy4 sequences, referred to as haplotypes. The overview of all haplotypes and the corresponding tomato accessions is presented in $\mathrm{S} 2-\mathrm{S} 4$ Tables. The upper part of Tables 1 - 3 separated by the broken line represents the haplotypes found predominantly in cultivated and landrace accessions, whereas the lower part shows the haplotypes of wild species accessions. More amino acid changes were found in protein sequences of wild species accessions than in those of cultivated and landrace accessions. The highest number of amino acid substitutions identified in wild accessions were seven, nine and five in SuSy1-haplotype\#23, SuSy3-haplotype\#22 and SuSy4-haplotype\#17, respectively; whereas the cultivated and landrace accessions had maximally three, two and two substitutions in SuSy1-haplotype\#7, SuSy3-haplotype\#4, and SuSy4-haplotype\#3, respectively. The identified variations in SuSy1/3/4 of 85 accessions were distributed over all domains identified for AtSuSyl: cellular target domain (CTD), ENOD40 peptide-binding domain (EPBD), GT-B glycosyltransferase domain (GT-B), and C-terminal extension (C), except for SuSy4, which had no variation in the $\mathrm{C}$ domain (Tables 1-3). Furthermore, the common motif $\mathrm{E}-\mathrm{X}_{7}-\mathrm{E}$, which is involved in the transfer of a glycosyl residue from a donor sugar to an acceptor, present in the glycosyltransferase family reported by Cid et al. (2000), was conserved in all these tomato SuSy haplotypes (see S2 File). 
Table 1. Tomato SuSy1 haplotypes (805aa)

\begin{tabular}{|c|c|c|c|c|c|c|c|c|c|c|c|c|c|c|c|c|c|c|}
\hline Domain & CT & & & & & GT & & & & & & & & & & & & $\mathrm{C}$ \\
\hline Position & n & $=$ & $m$ & $\stackrel{\circ}{\beth}$ & $\stackrel{\circ}{\sim}$ & $\frac{\infty}{m}$ & $\stackrel{\infty}{+}$ & 点 & $\stackrel{n}{n}$ & $\hat{n}$ & $\stackrel{8}{\circ}$ & î & 8 & $\tilde{\sigma}$ & $\vec{G}$ & $\widehat{N}$ & 요 & 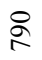 \\
\hline $\begin{array}{r}\text { Ref. } \\
\text { Haplo. }\end{array}$ & $\mathrm{E}$ & $\mathrm{R}$ & $\mathrm{S}$ & A & I & $\mathrm{L}$ & G & $\mathrm{V}$ & $\mathrm{R}$ & $S$ & $\bar{K}$ & $\mathrm{~T}$ & G & $\mathrm{N}$ & $\mathrm{R}$ & $\mathrm{E}$ & $\mathrm{K}$ & A \\
\hline $1(15)^{* \$}$ & . & . &. &. & . & . & . &. & . &. & . &. & . & . & . & . & . & . \\
\hline 2 & . & . & . & . & . & . & . & . & . & . & . & . & . & . & . & . & . & S \\
\hline $3(5)^{\$}$ & . & S & . & . & . & . & . & . & . & . & . & . & . & . & . & . & . & . \\
\hline $4(23)$ & . & . & . & . & . & . & . & G & . & . & . & . & . & . & . & . & . & \\
\hline $5(4)$ & . & S & . & . & . & . & . & $\mathrm{G}$ & . &. & . & . & . & . & . & . & . & . \\
\hline $6(8)$ & . & S & . & . & . & . & . & . & . & $\mathrm{T}$ & . & . & . & . & . & . & . & . \\
\hline 7 (12) & . & $\mathrm{S}$ & . & . & . & . & . & G & . & $\mathrm{T}$ & . & . & $\therefore$ & . & $\therefore$ & . & . & . \\
\hline 8 & 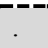 & $\mathrm{S}$ & 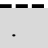 & . & 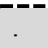 & 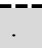 & . & 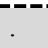 & . & $\mathrm{T}$ & . & . & . & . & $\mathrm{K}$ & . & . & . \\
\hline 9 & . & S & . & . & . & . & . & . & . & $\mathrm{T}$ & . & . & . & . & . & D & . & . \\
\hline 10 & . & S & . & . & . & . & . & . & $\mathrm{H}$ & $\mathrm{T}$ & . & . & . & . & . & . & . & . \\
\hline 11 & . & S & . & . & . & . & . & . & . & $\mathrm{T}$ & . &. & $\mathrm{E}$ & . & . & D & . & . \\
\hline 12 & . & S & . & . & . & . & . & . & . & $\mathrm{T}$ & . & S & . & . & . & . & $\mathrm{N}$ & \\
\hline $13(3)$ & . & $\mathrm{S}$ & . & . & V & . & . & . & . & $\mathrm{T}$ & . & . & . & . & . & . & . & \\
\hline 14 & . & S & . & . & V & . & . & G & . & $\mathrm{T}$ & . & . & . & . & . & . & . & \\
\hline 15 & . & S & . & $\mathrm{T}$ & . & . & . & $\mathrm{G}$ & . & $\mathrm{T}$ & . & . & . & . & . & . & . & \\
\hline 16 & . & S & . & $\mathrm{T}$ & . & . & . & . & . & $\mathrm{T}$ & . & S & . & . & . & . & . & \\
\hline 17 & . & S & . & . & . & . & . & . & . & $\mathrm{T}$ & . & S & . & . & . & . & $\mathrm{N}$ & . \\
\hline 18 & . & S & . & $\mathrm{T}$ & . & . & . & . & . & $\mathrm{T}$ & . & S & $\mathrm{E}$ & . & . & . & . & \\
\hline 19 & . & S & . &. & . & $\mathrm{F}$ & . & . & . & $\mathrm{T}$ & . & . & . & K & . & . & . & \\
\hline 20 & . & S & . & . & . & . & . & G & . & $\mathrm{T}$ & & S & . & . & . & . & $\mathrm{N}$ & \\
\hline 21 & D & S & . & . & . & . & $\mathrm{S}$ & . & . & $\mathrm{T}$ & . & . & . & . & . & . & . & \\
\hline 22 & . & S & . & . & . & . & S & G & . & $\mathrm{T}$ & . & . & . & . & . & . & . & . \\
\hline 23 & . & S & $\mathrm{T}$ & . & . & . & S & G & . & $\mathrm{T}$ & M & . & . & . & . & D & . & \\
\hline Effect $^{\#}$ & - & - & - & - & - & + & - & + & + & - & - & - & - & - & + & - & + & - \\
\hline
\end{tabular}

* The number of accessions in each haplotype is given between parentheses; the haplotypes without the parenthesis means that they only have one accession. " The amino acid substitution is predicted to have (+) or not to have (-) effect on protein function. ${ }^{\$}$ These haplotypes are chosen to be cloned and heterogously expressed. The dot "." indicates the same amino acid as the reference's at the corresponding position. The broken line separates the cultivated and landrace accessions from the wild species ones above and below the line, respectively. 
Table 2. Tomato SuSy3 haplotypes (805aa)

\begin{tabular}{|c|c|c|c|c|c|c|c|c|c|c|c|c|c|c|c|c|c|c|c|c|}
\hline \multirow{2}{*}{$\begin{array}{l}\text { Domain } \\
\text { Position }\end{array}$} & \multicolumn{4}{|c|}{ CTD } & \multicolumn{3}{|c|}{ EPBD } & \multicolumn{12}{|c|}{ GT-B } & \multirow{2}{*}{$\begin{array}{l}\mathrm{C} \\
\stackrel{\infty}{2}\end{array}$} \\
\hline & $\hat{n}$ & $\Re$ & $\infty$ & 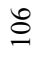 & $\infty$ & $\stackrel{\partial}{\partial}$ & $\frac{\partial}{\vec{\lambda}}$ & 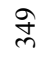 & $\vec{n}$ & ì & $n$ & in & in & ঠூర & ฮิ & సु & $\underset{N}{\mathbb{N}}$ & $\widehat{N}$ & 寺 & \\
\hline Ref. & $\mathrm{S}$ & $\mathrm{S}$ & I & $\mathrm{S}$ & $\mathrm{N}$ & Q & $\mathrm{S}$ & Q & $\mathrm{H}$ & $\mathrm{P}$ & $\mathrm{D}$ & $\mathrm{E}$ & W & $\mathrm{L}$ & $\mathrm{E}$ & $\mathrm{H}$ & $\mathrm{E}$ & $\mathrm{E}$ & K & $\mathrm{E}$ \\
\hline \multicolumn{21}{|l|}{ Haplo. } \\
\hline $1(50)^{* \$}$ & . & . & . & . & . & . & . & . & . & . & . & . & . & . & . & . & . & . & . & . \\
\hline 2 & . & . & . & . & . & . & . & . & . & . & $\mathrm{Y}$ & . & . & . & . & . & . & . & . & . \\
\hline $3(2)$ & . & . & . & I & . & . & . & . & . & . & . & . & . & . & . & . & . & . & . & . \\
\hline $4(8)$ & - & . & - & I & - & - & - & - & - & . & . & . & . & . & . & . & . & . & $\mathrm{E}$ & . \\
\hline 5 & . & . & . & I & . & . & . & . & $\mathrm{R}$ & . & . & . & . & . & . & . & . & . & $\mathrm{E}$ & . \\
\hline 6 & . & . & . & I & . & . & . & . & . & . & $\mathrm{Y}$ & . & . & . & . & . & . & . & $\mathrm{E}$ & . \\
\hline 7 & . & . & . & I & . & . & . & . & . & . & . & . & . & . & . & . & $\mathrm{D}$ & . & $\mathrm{E}$ & . \\
\hline $8(5)$ & . & . & . & I & . & $\mathrm{H}^{\mathrm{a}}$ & . & . & . & . & . & . & . & . & . & . & $\mathrm{D}$ & . & $\mathrm{E}$ & . \\
\hline $9(2)^{\$}$ & A & . & . & I & . & . & . & . & . & . & . & . & . & . & . & . & . & $\mathrm{D}$ & E & . \\
\hline $10(4)^{\$}$ & A & . & . & I & . & . & . & $\mathrm{L}$ & . & . & . & . & . & . & . & . & . & $\mathrm{D}$ & $\mathrm{E}$ & . \\
\hline 11 & A & . & . & I & . & . & . & . & . & . & . & $\mathrm{D}$ & . & . & . & . & . & $\mathrm{D}$ & $\mathrm{E}$ & . \\
\hline 12 & . & . & . & I & $\mathrm{K}$ & $\mathrm{L}^{\mathrm{b}}$ & . & . & . & . & . & . & . & . & . & . & $\mathrm{D}$ & $\mathrm{D}$ & $\mathrm{E}$ & . \\
\hline 13 & . & $\mathrm{C}$ & . & I & $\mathrm{K}$ & . & . & . & . & . & . & . & . & . & . & . & $\mathrm{D}$ & $\mathrm{D}$ & E & . \\
\hline 14 & . & $\mathrm{C}$ & . & I & $\mathrm{K}$ & . & . & . & . & . & . & . & . & . & . & . & $\mathrm{D}$ & . & E & . \\
\hline 15 & . & . & $\mathrm{F}$ & I & . & . & . & . & . & . & . & . & . & . & . & . & $\mathrm{D}$ & . & $\mathrm{E}$ & . \\
\hline 16 & . & . & . & I & . & . & . & . & . & $\mathrm{S}$ & . & . & . & . & G & . & $\mathrm{D}$ & . & $\mathrm{E}$ & . \\
\hline 17 & A & . & . & I & . & . & . & . & . & $\mathrm{S}$ & . & . & . & . & G & . & $\mathrm{D}$ & $\mathrm{D}$ & $\mathrm{E}$ & . \\
\hline 18 & . & . & . & I & . & . & . & . & . & $\mathrm{S}$ & . & . & . & . & . & . & $\mathrm{D}$ & $\mathrm{D}$ & $\mathrm{E}$ & $\mathrm{K}$ \\
\hline 19 & . & . & . & I & $\mathrm{K}$ & . & . & . & . & $\mathrm{S}$ & . & . & G & . & . & . & $\mathrm{D}$ & $\mathrm{D}$ & $\mathrm{E}$ & $\mathrm{K}$ \\
\hline 20 & . & . & . & I & $\mathrm{K}$ & $\mathrm{H}^{\mathrm{a}}$ & $\mathrm{P}$ & . & . & . & . & . & . & . & . & . & $\mathrm{D}$ & D & $\mathrm{E}$ & $\mathrm{K}$ \\
\hline 21 & . & . & . & I & $\mathrm{K}$ & . & . & . & . & . & . & . & . & I & . & $\mathrm{Y}$ & $\mathrm{D}$ & $\mathrm{D}$ & $\mathrm{E}$ & K \\
\hline 22 & A & . & . & I & $\mathrm{K}$ & $\mathrm{H}^{\mathrm{a}}$ & $\mathrm{P}$ & . & . & . & . & . & . & . & . & . & $\mathrm{D}$ & $\mathrm{D}$ & $\mathrm{E}$ & K \\
\hline Effect $^{\#}$ & - & - & + & - & - & $\begin{array}{l}-^{\mathrm{a}} \\
+^{\mathrm{b}}\end{array}$ & - & + & - & + & - & - & + & - & - & - & - & + & - & - \\
\hline
\end{tabular}

* The number of accessions in each haplotype is given between parentheses; the haplotypes without the parenthesis means that they only have one accession. " The amino acid substitution is predicted to have (+) or not to have (-) effect on protein function. ${ }^{\$}$ These haplotypes are chosen to be cloned and heterogously expressed. The dot "." indicates the same amino acid as the reference's at the corresponding position. The broken line separates where the cultivated and landrace accessions from the wild species ones above and below the line, respectively. At position 199, the amino acid substation $\mathrm{Q} 199 \mathrm{H}^{\mathrm{a}}$ and $\mathrm{Q} 199 \mathrm{~L}^{\mathrm{b}}$ is predicted to have neutral and deleterious on protein function, respectively. 
Table 3. Tomato SuSy4 haplotypes (812aa)

\begin{tabular}{|c|c|c|c|c|c|c|c|c|c|c|c|c|c|c|c|}
\hline Domain & CTD & & EPB & & & GT- & & & & & & & & & \\
\hline Position & $\ddot{m}$ & $\stackrel{\Xi}{\Xi}$ & $\stackrel{n}{\simeq}$ & $\stackrel{\sim}{\sim}$ & సે & $\vec{\infty}$ & $\stackrel{\sim}{\sim}$ & $\begin{array}{l}\infty \\
\stackrel{o}{+}\end{array}$ & $\bar{\delta}$ & $\begin{array}{l}n \\
\infty \\
n\end{array}$ & రై & $\tilde{\infty}$ & $\begin{array}{l}\infty \\
\infty \\
\infty\end{array}$ & 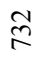 & $\stackrel{\infty}{\sim}$ \\
\hline Ref. & $\mathrm{V}$ & $\mathrm{V}$ & $\mathrm{N}$ & $\mathrm{N}$ & $\mathrm{D}$ & $\mathrm{N}$ & $\mathrm{K}$ & $\mathrm{V}$ & $\mathrm{S}$ & $\mathrm{V}$ & $\mathrm{L}$ & $\mathrm{V}$ & $\mathrm{T}$ & $\mathrm{R}$ & $\mathrm{T}$ \\
\hline \multicolumn{16}{|l|}{ Haplo. } \\
\hline $1(55)^{*}$ & . & . & . & . & . & . & . & . & . & . & . & . & . & . & . \\
\hline $2(5)$ & G & . & . & . & . & . & . & . & . & . & . & . & . & . & . \\
\hline $3(1)$ & . & . & . & . & . & . & . & G & . & G & . & . & . & . & . \\
\hline $4(5)$ & . & I & . & . & . & . & . & . & . & & . & . & . & . & . \\
\hline $5(2)$ & . & I & . & S & . & . & . & . & . & . & . & . & . & . & . \\
\hline 6 & . & I & . & . & E & . & . & . & . & . & . & . & . & . & . \\
\hline $7(2)$ & . & I & $\mathrm{S}$ & . & . & . & . & . & . & . & . & . & . & . & . \\
\hline 8 & . & I & . & . & . & . & . & . & $\mathrm{N}$ & . & . & . & . & . & . \\
\hline $9(2)$ & G & I & . & S & . & . & . & . & . & . & . & . & . & . & . \\
\hline $10(2)$ & G & I & S & . & . & . & . & . & . & . & . & . & . & . & A \\
\hline 11 & . & I & S & . & . & . & . & . & $\mathrm{N}$ & . & . & . & . & . & . \\
\hline $12(2)$ & . & I & . & . & E & . & . & . & $\mathrm{N}$ & . & . & . & . & . & . \\
\hline $13(2)$ & . & I & . & . & E & . & . & . & $\mathrm{N}$ & . & . & . & . & Q & . \\
\hline 14 & . & I & . & . & E & . & . & . & $\mathrm{N}$ & . & . & . & I & . & . \\
\hline 15 & . & I & . & . & E & . & . & . & $\mathrm{N}$ & . & . & $\mathrm{L}$ & I & . & . \\
\hline 16 & . & I & . & S & . & S & . & . & . & . & $\mathrm{F}$ & . & . & . & . \\
\hline 17 & $\mathrm{G}$ & I & $\mathrm{K}$ & . & . & . & $\mathrm{N}$ & . & $\mathrm{N}$ & . & . & . & . & . & . \\
\hline Effect $^{\#}$ & + & - & - & - & - & + & - & - & - & + & + & + & + & - & - \\
\hline
\end{tabular}

* The number of accessions in each haplotype is given between parentheses; the haplotypes without the parenthesis means that they only have one accession. " The amino acid substitution is predicted to have $(+)$ or not to have (-) effect on protein function. The dot "." indicates the same amino acid as the reference's at the corresponding position. The broken line separates where the cultivated and landrace accessions from the wild species ones above and below the line, respectively.

\section{Found natural allelic variation is predicted to have no effect on protein structure}

A. thaliana SuSy1 (PDB 3S27, 3S28 and 3S29 , 808 aa) (Zheng et al. 2011) is the only plant SuSy protein with the crystal structure resolved till now and thus was used as a template to generate tomato SuSy models in order to predict the effect of identified amino acid variations on the structures. A protein sequence alignment between AtSuSyl and haplotype\#1 of tomato SuSy1/3/4 shows that tomato SuSy1 and SuSy3 have the highest homology to AtSuSy1 with $78 \%$ and $77 \%$ identity respectively, while it is $68 \%$ for SuSy 4 (data not shown). According to one of the criteria of template-based protein structure modeling, the sequence identity between the query (e.g. tomato SuSy) and template (e.g. AtSuSy1) should be at least $30 \%$ to obtain a model with sufficient accuracy (Fiser 2010). Therefore the protein structures of all the tomato SuSy1/3/4 haplotypes are predicted with high confidence using AtSuSyl as the template using the Phyre2 modeling suite. All the found variations in tomato SuSy1/3/4 haplotypes are predicted not to cause disruption of $\alpha$-helixes or $\beta$-strands in secondary structure compared to that of the reference cultivar Heinz (data not shown). Figure 1A shows 
an example of a subunit of the predicted tomato SuSyl-haplotype\#1 3D structure model showing 781 residues (97\% of the peptide sequence). Furthermore, based on the homotetrameric protein structure of AtSuSyl (Zheng et al. 2011), none of the amino acid changes found in SuSys of 85 tomato accessions are predicted to be involved in subunit interactions (Fig 1B, S1 File and S1 Fig.). The corresponding residues involved in A:D subunit interaction of tomato SuSy1/3 are $129-140$ and $776-794$ and that of tomato SuSy4 are 132 -143 and $779-799$. The residues $145-152$ of tomato SuSyl/3 and residues $148-155$ of tomato SuSy4 of the adjacent EPDBs are involved in A:B subunit interaction.
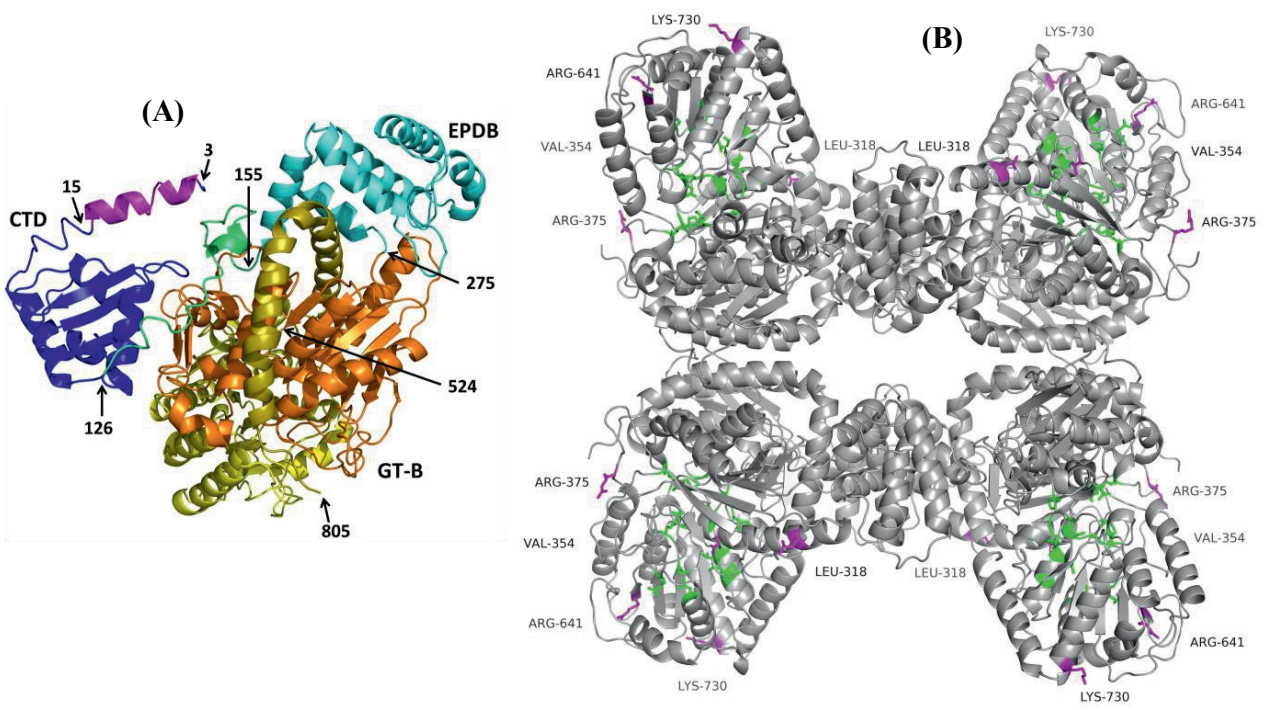

Fig 1. Ribbon drawings of the 3D modelled tomato SuSy1 haplotype\#1 monomer and homotetramer. Based on the structure of AtSuSy1 (PBD ID 3S18), the monomer structure (A) starts with the cellular targeting domain colored blue (CTD, residues 3-125) containing a $\alpha 1$ helix (magenta, residue 3-15). A linker colored green (residues 126-154) bridges the CTD with the ENOD40 peptidebinding domain in cyan color (EPDB, residue 155-274). The structure continues with a GT-B glycosyltranferase domain (GT-B) which is divided into GT- $\mathrm{B}_{\mathrm{N}}$ domain in orange (residues 275-523) and $\mathrm{GT}-\mathrm{B}_{\mathrm{C}}$ domain in yellow (residues 524-771). This monomer ends with a $\mathrm{C}$-terminal extension domain (C) (residues 773-805). In the homo-tetramer model (B), the amino acid residues interacting with substrate are indicated in green, while the residues L318, V354, R375, R641, and L730 with variation in SuSyl are highlighted in magenta. All these amino acid are not close to the binding sites, or in the interfaces between the subunits. 


\section{Substrate binding sites and phosphorylation sites are highly conserved}

Functional sites of tomato SuSy proteins such as substrate binding sites and phosphorylation sites were predicted (S1 File), based on the known substrate binding sites identified in AtSuSyl (Zheng et al. 2011). The substrate binding sites were clustered in GT-B domain and conserved in 85 tomato accessions. All the positions with amino acid variation in SuSy1/3/4 were predicted to be more than $10 \AA$ away from the substrate binding sites and stick out to the solvent. Figure 1B shows an example of the homo-tetramer model of tomato SuSyl displaying five residues with variation that are not in contact with the substrate binding sites and stick out to the solvent. In maize, the SuSy sites Ser-15 and Ser-170 have been reported as two conserved phosphorylation sites (Hardin et al. 2003, 2004b). These two sites were also conserved in all tomato SuSy3 protein variants at corresponding positions 11 and 165 and in SuSy4 at positions 11 and 168. In SuSyl, the second phosphorylation site Ser-165 was conserved in all the studied accessions, but the first phosphorylation site Ser-11 was not. This phosphorylation site Ser-11 was present in 30 out of 32 wild species accessions, whereas only less than half, 22 out of 53, cultivated and landrace accessions contained this site (Table 1).

\section{Several variations in SuSy are predicted to affect protein function}

The potential effect of amino acid changes on protein function was also investigated in silico. As shown in Table 1-3, the majority of amino acid changes was predicted to not to have an impact on protein function in the studied SuSys. However, several amino acid substitutions in each SuSy isoform were predicted to have an effect on protein function, which could be substantially lower or higher enzyme activity, or result into a profound change in enzyme kinetics. In SuSy1, variation L318F, V354G, R375H, R641K, and K730N were predicted to affect protein function, in which most of them were only found in wild species accessions, except V354G (Table 1). In SuSy3 variation I88F, Q199L, P546S, W591G, and E727D were predicted to affect protein function, and they were again only identified in wild species accessions (Table 2). In SuSy4 six variations were predicted to affect SuSy4 function, in which V35G, N281S, L634F, V683L, and T688I were found in wild species and V585G in cultivated and landrace accessions (Table 3 ).

\section{The tested SuSy3-haplotype\#9 protein variant from wild accession has slightly higher sucrose-hydrolyzing capacity compared to the control from cultivated tomato}

To gain insight in the role of identified natural variation on enzyme kinetics, two SuSyl and three SuSy3 protein variants were chosen to be expressed in yeast host $S$. cerevisiae strain $22574 \mathrm{~d}$ and purified for further study. The first step of enzyme purification using ion exchange chromatography (IEX) appeared to be most efficient with SuSy3 protein variants, less efficient with SuSyl protein variants, and not successful with SuSy4 protein variants (S2 - S3 Fig.). The recombinant protein variants were tested for their maximal velocity in hydrolyzing sucrose $\left(\mathrm{V}_{\max }\right), \mathrm{K}_{\mathrm{m}}$ for sucrose, and catalytic efficiency $\left(\mathrm{V}_{\max } / \mathrm{K}_{\mathrm{m}}\right)$ at both $25^{\circ} \mathrm{C}$ and $16^{\circ} \mathrm{C}$. For $\mathrm{SuSy} 3$, we focused on the haplotypes of two high-altitude wild accessions: $S$. arcanum LA385, representing haplotype\#9, and S. neorickii LA0735 (also known as $S$. neorickii G1.1601), representing haplotype\#10, collected at 2,500 and 2,900 meters above sea 
level respectively (UC Davis Tomato Genetics Resource Center). As shown in Table 4, low temperature had an expected impact on enzyme kinetics of all tested SuSy3 protein variants. At low temperature $\left(16^{\circ} \mathrm{C}\right)$ the $\mathrm{V}_{\max }$ of all three tested $\mathrm{SuSy} 3$ protein variants was reduced to about $55 \%$ to $75 \%$ of the values found at $25^{\circ} \mathrm{C}$. The $\mathrm{K}_{\mathrm{m}}$ values of these SuSy3 protein variants were about two- to three-fold higher at $16^{\circ} \mathrm{C}$ compared to the values found at $25^{\circ} \mathrm{C}$. As a consequence, the $\mathrm{V}_{\max } / \mathrm{K}_{\mathrm{m}}$ values of these SuSy3 protein variants were about four-fold lower at $16^{\circ} \mathrm{C}$ compared to those at $25^{\circ} \mathrm{C}$. SuSy3-haplotype\#9 showed slightly improved kinetics with higher $\mathrm{V}_{\max }$ and $\mathrm{V}_{\max } / \mathrm{K}_{\mathrm{m}}$ values at both temperatures relatively to the reference SuSy3-haplotype\#1. Because SuSy3-haplotype\#10 had lower purity (i.e. only purified by IEX) compared to the variants of haplotype\#1 and haplotype\#9 (i.e. purified by IEX and IMAC), it was not possible to make the comparison for the enzyme kinetics values between them.

Table 4. Enzyme kinetic parameters

$\begin{array}{llll}\text { Assay } \mathrm{T} & \mathrm{V}_{\max } & \mathrm{K}_{\mathrm{m}} & \mathrm{V}_{\max } / \mathrm{K}_{\mathrm{m}}\end{array}$

$\left({ }^{\circ} \mathrm{C}\right) \quad\left(\mathrm{U} \mathrm{mg}^{-1}\right) \quad(\mathrm{mM} \mathrm{suc}) \quad\left(\mathrm{U} \mathrm{mg}^{-1} \quad \mathrm{M}^{-1}\right)$

\begin{tabular}{|c|c|c|c|c|}
\hline \multirow[t]{2}{*}{ SuSy1-haplotype $\# 1^{\$}$} & $25^{\circ} \mathrm{C}$ & $1.2 \pm 0.1$ & $51 \pm 13$ & $24 \pm 8$ \\
\hline & $16^{\circ} \mathrm{C}$ & $0.7 \pm 0.3$ & $189 \pm 90$ & $5 \pm 1^{\mathrm{a}}$ \\
\hline SuSy1-haplotype\#3 ${ }^{\$}$ & $25^{\circ} \mathrm{C}$ & $1.4 \pm 0.2$ & $56 \pm 14$ & $26 \pm 3$ \\
\hline (R11S) & $16^{\circ} \mathrm{C}$ & $0.8 \pm 0.2^{a}$ & $101 \pm 15^{\mathrm{a}}$ & $8 \pm 3^{b}$ \\
\hline \multirow[t]{2}{*}{ SuSy3-haplotype\#1 } & $25^{\circ} \mathrm{C}$ & $4.6 \pm 0.5$ & $34 \pm 8$ & $142 \pm 38$ \\
\hline & $16^{\circ} \mathrm{C}$ & $2.6 \pm 0.4^{b}$ & $77 \pm 22^{a}$ & $36 \pm 7^{b}$ \\
\hline SuSy3-haplotype\#9 ${ }^{\#}$ & $25^{\circ} \mathrm{C}$ & $6.5 \pm 1.2$ & $33 \pm 9$ & $206 \pm 50$ \\
\hline (S53A, S106I, E727D, K741E) & $16^{\circ} \mathrm{C}$ & $3.9 \pm 1.3^{\mathrm{a}}$ & $75 \pm 19^{a}$ & $52 \pm 5^{\mathrm{c} *}$ \\
\hline SuSy3-haplotype\#10\$ & $25^{\circ} \mathrm{C}$ & $6.2 \pm 0.7$ & $58 \pm 14$ & $112 \pm 37$ \\
\hline (S53A, S106I, E727D, Q349L, K741E) & $16^{\circ} \mathrm{C}$ & $4.6 \pm 1.2$ & $167 \pm 29^{a}$ & $28 \pm 6^{a}$ \\
\hline
\end{tabular}

${ }^{\$}$ These proteins were purified with IEX; ${ }^{\#}$ These proteins were purified with IMAC. Data represent the mean of three independent measurements $( \pm$ SD). Significant differences are labelled with $\mathrm{a}, \mathrm{b}$ and $\mathrm{c}$ for $P<0.05,0.01$ and 0.001 between assay temperatures of each purified enzyme, respectively. The significant difference between different SuSy1/3 protein variants and the corresponding protein from the reference haplotype\#1 is denoted as * for $P<0.05$. 


\section{No difference in enzyme kinetics between SuSy1 protein variants with amino acid alterations at the first phosphorylation site}

The effect of amino acid alterations in the first phosphorylation site of SuSy1 protein was investigated by studying enzyme kinetic parameters of SuSy1-haplotype\#3 containing the phosphorylation site Ser-11 and SuSyl-haplotype\#1 with Arg-11. Low temperature had the same impact on enzyme kinetics of both SuSyl protein variants as shown in SuSy3 protein variants (Table 4). The $\mathrm{V}_{\max }$ and $\mathrm{K}_{\mathrm{m}}$ values for both SuSyl variants was within error identical at $25^{\circ} \mathrm{C}$, but the $\mathrm{K}_{\mathrm{m}}$ of SuSy1-haplotype\#1 increased much more compared to that of SuSy1haplotype $\# 3$ at $16^{\circ} \mathrm{C}$ (i.e. $50 \mathrm{mM}$ to $189 \mathrm{mM}$ compared to $60 \mathrm{mM}$ to $100 \mathrm{mM}$, respectively). Similarly to SuSy3 protein variants, the $\mathrm{V}_{\max } / \mathrm{K}_{\mathrm{m}}$ values of these SuSy1 protein variants were also about four-fold lower at $16^{\circ} \mathrm{C}$ compared to those at $25^{\circ} \mathrm{C}$. However, there was no difference in the $\mathrm{V}_{\max } / \mathrm{K}_{\mathrm{m}}$ value between SuSy1-haplotype\#1 and SuSy1-haplotype\#3 at both temperatures.

\section{Discussion}

\section{Wild accessions contain ample variation in SUSYs}

The current study focused on exploring the natural genetic variation of three distinct tomato SuSy1, Susy3, and Susy4 genes which might confer an improvement in sucrose hydrolyzing capacity. We observed the narrow genetic basis within the three SuSy genes of 53 cultivated and landrace accessions, whereas the number of SNPs leading to amino acid changes within SuSy1/3/4 of wild species accessions was 2.3, 4.5, and 2.5 fold higher than the most diverse counterpart from the cultivated and landrace set (Table 1-3). This genetic erosion in cultivated tomatoes is well known, which is due to the selection for a limited number of morphological and agronomical traits such as fruit shape and size, fruit color, self-pruning, plant height and earliness (Bai and Lindhout 2007, Rodríguez et al. 2011, Bauchet and Causse 2012). On the other hand, the wide array of genetic diversity found in wild species is in agreement with the report from Aflitos et al. (2014). Therefore, this observed ample variation within the studied wild species could be exploited to enhance the enzyme kinetics of SuSys.

\section{The known active sites of SUSYs are conserved}

Amino acid changes in active sites can have direct impact on enzyme kinetics. However, the in silico analysis showed that all the substrate binding sites found in AtSuSyl (Zheng et al. 2011) were also conserved in all three SuSy isoforms of 85 studied tomato accessions (S1 File). These active sites of SuSys are also conserved in other crops such as maize, rice, wheat, and even in non-photosynthetic bacteria Nitrosomonas europaea (S1 File; Volpicella et al. 2016). This means that the residues at those active sites are crucial for enzyme activity, which was confirmed by several reports. For instance, Wu et al. (2015) reported a significant loss of activity in sucrose synthesis of SuSy2 of the bacteria Nitrosomonas europaea when one of the conserved active site residues R567 and K575 (corresponding to R577 and K584 in 
tomato SuSy1/3) was altered to an Ala residue. Huang et al. (2016) also observed a profound decrease of sucrose binding ability in the recombinant rice SuSy3 mutants in which three different single amino acid changes in the conserved motif $\mathrm{E}-\mathrm{X}_{7}-\mathrm{E}$ were generated by sitedirected mutagenesis. This conserved motif corresponded to E673- $\mathrm{X}_{7}-\mathrm{E} 681$ in tomato SuSy1/3. As mentioned earlier, the sucrose binding site has not been determined experimentally (Zheng et al. 2011), therefore the residues involved in sucrose binding were postulated as the ones interacting with glucose and fructose moiety via the complexes AtSuSyl $\bullet U D P$-glucose and AtSuSyl $\bullet U D P \bullet$ fructose, respectively. These residues postulated to interact with sucrose could be confirmed by generating several inactive mutant variants of AtSuSy1, so that it can halt the fast sucrose processing. As a result, this approach could help to observe the sucrose binding to the active site in the SuSy enzyme. Several studies have successfully identified sucrose binding site by using such approach such as levansucrase of bacteria like Bacillus substilis and cell wall invertase of $A$. thaliana (Meng and Futterer 2003, Lammens et al. 2008).

\section{Recombinant SuSy3 of wild species shows an increase in sucrose hydrolyzing capacity}

The aim of this study was to find the SuSy protein variants among the landrace and wild accessions having improved kinetics at different temperatures compared to that of the control cultivated tomato. Even though no variation was found in the SuSy active sites as discussed above, the potential of enhancing SuSy kinetics was seen in SuSy3-haplotype\#9 of high altitude wild species $S$. arcanum LA385. SuSy3-haplotype\#9 was able to break down sucrose more efficiently (i.e. higher catalytic efficiency $\mathrm{V}_{\max } / \mathrm{K}_{\mathrm{m}}$ value) than SuSy3-haplotype\#1 of cv Moneymaker, especially at lower temperature of $16^{\circ} \mathrm{C}$ (Table 4). This accession LA385 has been reported to be less affected when grown at sub-optimal temperature of $16^{\circ} \mathrm{C}$ compared to two other commercial tomato cultivars, for instance less reduction of relative shoot growth rate and photosynthetic rate at both growth irradiation of $225 \mathrm{~mol} \mathrm{~m}^{-2} \mathrm{~s}^{-1}$ and light saturation of 1,200 mol m $\mathrm{m}^{-1}$ (Venema et al. 1999b). Whether SuSy3 would play a role in better performance of this accession LA385 at sub-optimal temperature is not known because the authors Venema et al. (1999b) only used younger plants at vegetative stage in their study, while SuSy3 expression and SuSy activity are found to be highest in fruits (Stommel 1992, Kortstee et al. 2007). The authors Kortstee et al. (2007) also pointed out that SuSy activity in fruits is the total sum of the activity of two main isoforms SuSyl and SuSy3, and it does not appear to correspond to the changes in gene expression when comparing those of the commercial tomato cultivar and the wild accession LA385. It will be interesting to study the effects of the introgression of such wild SuSy3 allele into a commercial tomato on plant growth, development and yield at standard and sub-optimal growth temperatures. As for the impact of amino acid changes on protein functionality, all four amino acid changes between SuSy3-haplotype\#1 and haplotype\#9, as well as those found in all other SuSy protein variants in this study, are predicted to be not close to the active sites and stick out to the solvent (Fig. 1 and S1 Fig.). Therefore, based on protein modeling, it is still unknown how the amino acid changes in these two haplotypes could influence the catalytic efficiency. However, it has been shown that amino acid substitutions far from the active site could also drastically affect enzymatic activity (Freeman et al. 2011). Therefore experiments, such as 
enzyme kinetic assays, are necessary to validate the prediction of impact of amino acid changes on protein function, and to investigate the possible underlying causes of observed impacts.

\section{The presence of major N-terminal phosphorylation site in SuSy1 does not improve affinity for sucrose}

Phosphorylation of SuSy has been reported to play an important role in its subcellular distribution and activity (Hardin et al. 2006). This study showed that SuSy1, also known as TOMSSF, from many cultivated tomato accessions lacks the major N-terminal phosphorylation site Ser-11 (Table 2 and S2 Table), supporting the finding of previous work (Wang et al. 1993a). On the other hand, this major phosphorylation site in SuSyl is present amongst most of the studied wild accessions and almost half of the cultivated and landrace accessions. The result with IEX-purified SuSyl protein variants showed that SuSy1haplotype\#3 containing Ser-11 did not show an improvement of enzyme kinetics at both temperatures compared to that of SuSyl-haplotype\#1 containing Arg-11, i.e. no clear difference in $\mathrm{V}_{\max }, \mathrm{K}_{\mathrm{m}}$ for sucrose, and catalytic efficiency $\mathrm{V}_{\max } / \mathrm{K}_{\mathrm{m}}$. The phosphorylation status of the recombinant SuSy1-haplotype\#3 containing the phosphorylation site Ser-11 was questionable, but the study of Sauerzapfe et al. (2008) could help to answer this question. Those authors used $\mathrm{Fe}^{3+}$ metal affinity chromatography ( $\mathrm{Fe}^{3+}$-IMAC) to demonstrate that their potato SuSyl was phosphorylated when produced by the same yeast host $S$. cerevisiae strain $22574 \mathrm{~d}$ as heterologous expression system and protein purification method as in our study. Those authors showed that the phosphorylated protein was eluted at higher $\mathrm{pH}$ compared to the dephosphorylated variant and another recombinant SuSyl with S11A substitution. $\mathrm{Fe}^{3+}$-IMAC has been reported to be highly sensitive, in which one phosphate group within the protein is enough to bind to the immobilized $\mathrm{Fe}^{3+}$ ions and the elution towards more alkaline pH (Muszynska et al. 1986, Muszyńska et al. 1992). At the protein level tomato SuSy1-haplotype\#3 and the aforementioned potato SuSy1 are 98\% identical, and both contain the conserved phosphorylation site Ser-11 (data not shown). In addition, Anguenot et al. (1999) has also shown that $\mathrm{Fe}^{3+}$-IMAC could separate the tomato fruit SuSy non-phosphorylated protein and the phosphorylated ones. Interestingly, our results in tomato were contrasting with the findings of Sauerzapfe et al. (2008) in potato, who reported that the recombinant potato SuSy1 with S11A substitution (IEX-purified) had an 8.5-fold increase in affinity for sucrose and a 300-fold decrease in $\mathrm{V}_{\max }$ compared to that in its counterpart with phosphorylation site Ser-11 (IMAC-purified). It appears that SuSy1 kinetic properties can be impacted severely when the phosphorylation site Ser-11 is substituted to non-polar side chain residue such as Ala (Sauerzapfe et al. 2008), compared to the change to positively charged residues like Arg in this study. In the same work, the authors Sauerzapfe et al. also studied the mutant SuSyl with S11D substitution, which was to imitate the phosphorylated site, and found that its affinity to sucrose remained the same level compared to the wildtype but its $\mathrm{V}_{\max }$ reduced significantly. This meant both substitutions S11A and S11D both drastically reduced the enzyme catalytic efficiency $\left(\mathrm{V}_{\max } / \mathrm{K}_{\mathrm{m}}\right)$, and that phosphorylation of residue Ser11 has a positive impact on the enzyme kinetics properties. On the other hand, in the available tetramer structures from AtSuSy1, the first $26 \mathrm{~N}$-terminal amino acid residues were 
not resolved, thus, no structural information is available yet to examine the position of this corresponding phosphorylated residue. Further experiments are needed to shed light on this phosphorylation mechanism.

\section{Conclusion}

Our study shows that genetic diversity in SuSy alleles of cultivated and landrace tomatoes is very limited, but a wide range of natural variation is found in the studied wild accessions. The enzyme kinetics study using several SuSy variants shows that SuSy3-haplotype\#9 containing four amino acid changes has an enhanced catalytic efficiency compared to the reference SuSy3-haplotype\#1, which is clearer at the assay temperature of $16^{\circ} \mathrm{C}$ than $25^{\circ} \mathrm{C}$. As a result, it would be interesting to investigate the effect of introgression of such a SuSy variant with enhanced catalytic efficiency into a commercial cultivar on plant sink strength, growth, and yield at different growth temperatures. Further research is needed to get better insights in the natural variation and to investigate the potential of the three extra predicted alleles $S u S y 5$, SuSy6, and SuSy7 to improve growth and development in tomato.

\section{Acknowledgments}

The authors thank Jos Molthoff for providing tomato samples, Dr. Richard Immink for advice on protein purification, and Dr. Anne Kortstee for providing plasmids. 


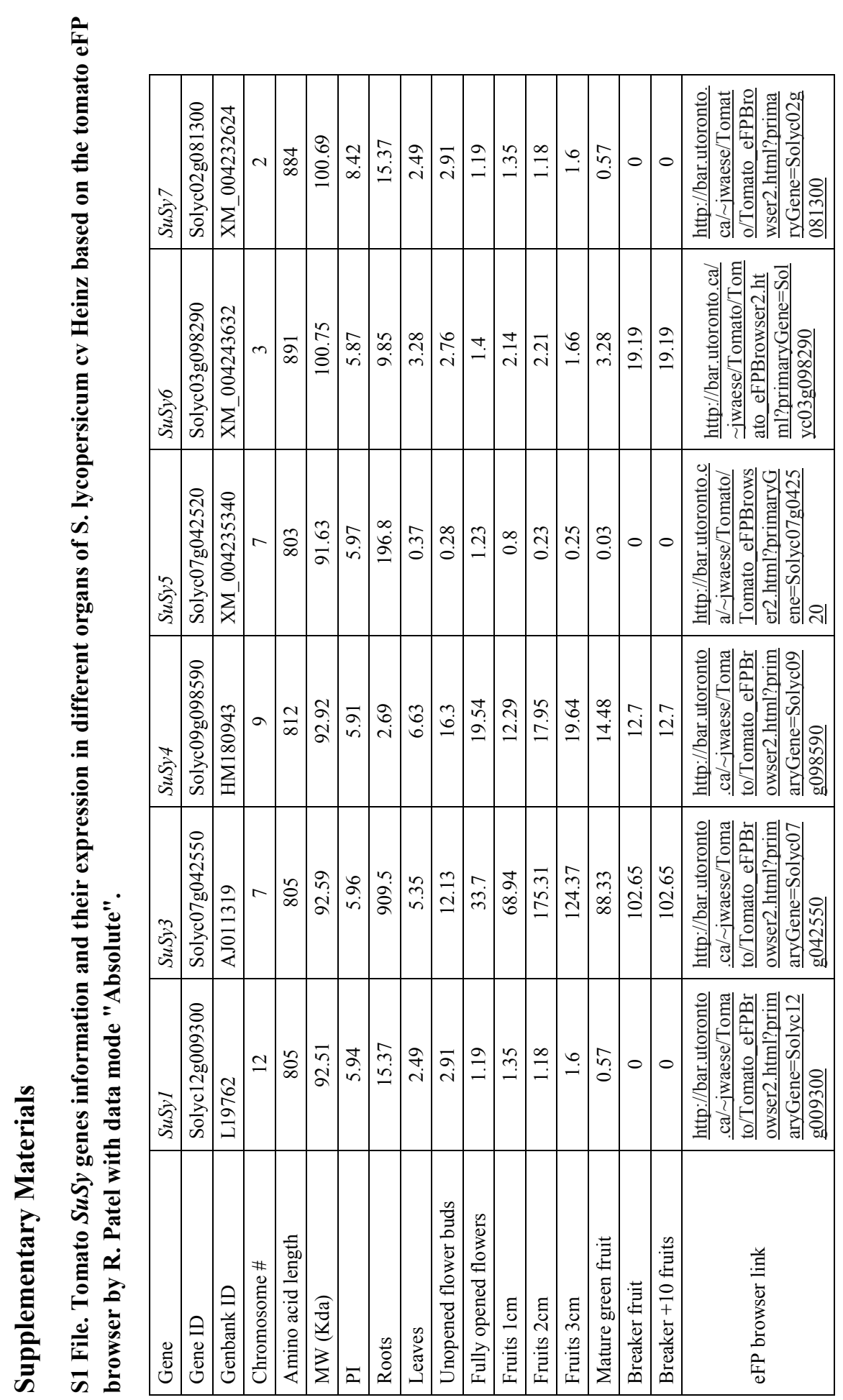




\section{S2 File. Sequence alignment of tomato SuSy1/3/4 haplotype\#1 against AtSuSy1 and SuSy from other species}

The conserved sites and motifs in the tomato SuSy1/3/4 isoforms are identified via the alignment with Arabidopsis SuSy1 (AtSuSy1, GenBank: OAO94355), rice SuSy3 (OsSuSy3, GenBank: ABL74561), maize SuSy1 (ZmSuSy1, GenBank: ACG43170), and SuSy2 from non-photosynthetic bacteria Nitrosomonas europaea (NeSuSy2, GenBank: 4RBN_A). The green, blue, red, dark red and purple lines cover different domains of SuSy as identified in AtSuSy1: cellular target domain (CTD), ENOD40 peptide-binding domain (EPBD), GT-B glycosyltransferase domain including $G T-B_{N}$ domain and $G T-B_{C}$ domain, and C-terminal extension (C), respectively. The cyan-highlighted residues are within $5 \mathrm{~A}^{\circ}$ distance from the substrates, whereas the pinkish-highlighted ones are within $10 \mathrm{~A}^{\circ}$ distance away from the substrates as measured with the $\mathrm{Cn} 3 \mathrm{D}$ application (https://www.ncbi.nlm.nih.gov/Structure/CN3D/cn3d.shtml). The residues with small black triangles are the substrate binding sites identified in AtSuSyl (Zheng et al. 2011), which are all conserved in three tomato SUS isoforms. In addition, tomato SUS isoforms also contain the conserved E-X $\mathrm{X}_{7}$-E motif (Cid et al. 2000) among the glycosyltransferase family enzyme, located at the residues 675-683 and highlighted with the orange line. Most of tomato SuSy also have two conserved phosphorylation sites, seen as red highlighted Ser-13 and Ser-167 in AtSuSy1. The grey-highlighted residues in tomato SuSy isoforms are the ones with natural variations among different studied accessions. For the subunit interaction found in AtSuSy1, the A:B interface was created by interaction between adjacent EPBDs and also residues 147 154 highlighted by the tan line. Meanwhile the A:D interface consists of the interaction between residues 131-142 and 778-796, which is highlighted by the black line. 
CTD

AtSUSY1

OSSUSY3

ZMSUSY1

SISUSY1

SISUSY3

SISUSY 4

NeSUSY2

AtSUSY1

OSSUSY3

ZMSUSY1

SISUSY1

SISUSY3

SISUSY 4

NeSUSY2

AtSUSY1

OSSUSY3

ZmSUSY1

SISUSY1

SISUSY3

SISUSY4

NeSUSY2

AtSUSY1 OSSUSY3

ZmSUSY1

SISUSY1

SISUSY3

SISUSY4

NeSUSY2

AtSUSY1 OSSUSY3 ZmSUSY1 SISUSY1 SISUSY 3 SISUSY4 NeSUSY2

AtSUSY1 OSSUSY 3 ZMSUSY1 SISUSY1 SISUSY 3 SISUSY 4 NeSUSY2

--MANAERMITRVHSQRERLNETLVSERNEVLALLSRVEAKGKGILQQNQIIAEFE-ALP MGETTGERALTRLHSMRERIGDSLSAHTNELVAVFSRLVNQGKGMLQPHQI IAEYNAAIP MGEGAGDRVLSRLHSVRERIGDSLSAHPNELVAVFTRLKNLGKGMLQPHQI IAEYNNAIP - --MAERVLTRVHRLRERVDATLAAHRNEILLFLSRIESHGKGILKPHELLAEFD-AIR ----MAQRVLTRVHSLRERLDATLDAHRNE ILLFLSRIESHGKGILKPHQLLAEFE-SIQ ----MSNPKLSRIPSMRERVEDTLSAHRNQLVALLSRYVAQGKGILQPHHLIDELNNAVC --------MTTI----DTFTTCTLQNRDAVYTLLRRYFSTNRPLLLQSDLRDELLQL-$:: \quad: \quad: \quad . \quad$. : : $\quad .: * \quad .::^{*} \quad .: \quad *$

EQ-TRKKLEGGPFFDLLKSTQEAIVLPPWVALAVRPRPGVWEYLRVNLHALVVEELQPAE EG-EREKLKDSALEDVLRGAQEAIVIPPWIALAIRPRPGVWEYLRINVSQLGVEELSVPE EA-EREKLKDGAFEDVLRAAQEAIVI PPWVALAIRPRPGVWEYVRVNVSELAVEELRVPE QD-DKDKLNEHAFEELLKSTQEAIVLPPWVALAIRLRPGVWEYVRVNVNALVVEELSVPE KE-DKDKLNDHAFEEVLKSTQEAIVLPPWVALAIRLRPGVWEYVRVNVNALSVEELTVPE DDTACEKLKEGPFCEILKSTQEAIVLPPFVAIAVRPRPGVWEYVRVNVYDLSVEOLTVPE ---EKDCERSDMLHEFVFHLQEGVFS SPWAYFVLRPGIAELEFVRMHQEHLMPEKITINE

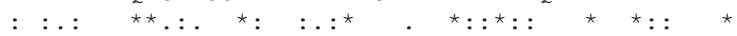

\section{A:B interface}

FLHFKEELVDGVKN-GNFTLELDFEPFNAS I PRPTLHKY I GNGVDFLNRHLSAKLFHDKE YLQFKEQLVDGSTQ-NNFVLELDFEPFNASFPRPSLSKS I GNGVQFLNRHLS SKLFHDKE YLQFKEQLVEEGPN-NNFVLELDFEPFNASFPRPSLSKS I GNGVQFLNRHLSSKLFHDKE YLQFKEELVDGASN-GNFVLELDFEPFTASFPKPTLTKSIGNGVEFLNRHLSAKMFHDKE FLQFKEELVNGTSS-DNFVLELDFEPFTASFPKPTLTKS IGNGVEFLNRHLSAKMFHDKE YLRFKEELVDGEDHNHLFVLELDFEPFNASVPRPSRSSS IGNGVQFLNRHLSSNMFRSNE FLGFKETVTKGEAI--ES I LEVDFGPFNRAFPKLRESRS I GQGVIFLNRQLSSEMFTRIE

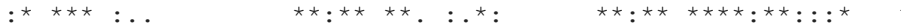

S-LLPLLKFLRLHSHQGKNLMLSEKIQNLNTLQHTLRKAEEYLAELKSETLYEEFEAKFE S-MYPLLNFLRAHNYKGMTMMLNDRIRS LDALQGALRKAEKHLAGITADTPYSEFHHRFQ S-MYPLLNFLRAHNYKGMTMMLNDRIRSLSALQGALRKAEEHLSTLQADTPYSEFHHRFQ S-MAPLLEFLRAHHYKGKTMMLNDRIHNSNTLQNVLRKAEEYLIMLPPETPFFEFEHKFQ S-MTPLLEFLRVHHYNGKSMMLNDRIQNLYTLQKVLRKAEEYLTTLSPETSYSSFEHKFQ S-LDPLLDFLRGHNHKGNVLMLNERIQRISRLESSLNKADDYLSKLPPDTPYTDFEYALQ AGSTRLLHFLGVHT I DGQQLMFT SNSHNINMVRSQLRQALEMLEAVDGTTPWAELSSDMS

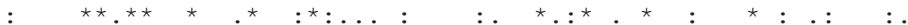
$\mathrm{GT}-\mathrm{B}_{\mathrm{N}}$

EIGLERGWGDNAERVLDMIRLLLDLLEAPDPCTLETFLGRVPMVFNVVILSPHGYFAQDN ELGLEKGWGDCAQRVRETIHLLLDLLEAPEPSALEKFLGTI PMVFNVVILSPHGYFAQAN ELGLEKGWGDCAKRAQET I HLLLDLLEAPDPSTLEKFLGTI PMVENVVI LSPHGYFAQAN EIGLEKGWGDTAERVLEMVCMLLDLLEAPDSCTLEKFLGRI PMVFNVVILSPHGYFAOEN EIGLERGWGDTAERVLEMICMLLDLLEAPDSCTLEKFLSRIPMVFNVVILSPHGYFAQEN EMGFEKGWGDTANRVLETMHLLSDILOAPDPSTLETFLGRLPMVFNVVILSPHGYFGQAN KIGFAPGWGHNAARVAETMNMLMDILEAPSPSALEAFLARI PMISRLLILSPHGYFGQDN

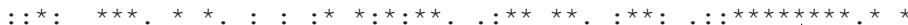
$\Lambda$

LGYPDTGGQVVYILDQVRALE IEMLQRIKQQGLNIKPRI LILTRLLPDAVGTTCGERLE VLGYPDTGGQVVY I LDQVRAMENEMLLRIKQQGLNITPRILIVTRLLPDAHGTTCGQRLE VLGYPDTGGQVVY ILDQVRAMENEMLLRIKQCGLDITPKILIVTRLLPDATGTTCGQRLE VLGYPDTGGQVVYILDQVPALEREMLKRIKEQGLDI I PRILIVTRLLPDAVGTTCGQRLE VLGYPDTGGQVVYILDQVPALEREMLKRIKEQGLDIKPRILIVTRLLPDAVGTTCGQRLE VLGLPDTGGQVVYILDQVRALEAEMLLRIKQQGLNFKPRILVVTRLI PDAKGTTCNQRLE VLGLPDTGGQVVY I LDQVRALEQEMRDRLQLQGVQVEPKILIVTRLI PDAGDTTCNQRLE

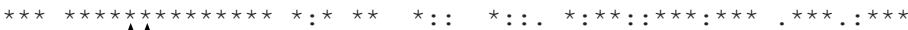


AtSUSY1

OSSUSY3

ZmSUSY1

SISUSY1

SISUSY3

SISUSY4

NeSUSY2

AtSUSY1 OsSUSY3 ZMSUSY1 SISUSY1 SISUSY 3 SISUSY4 NeSUSY2

AtSUSY1 OSSUSY3 ZmSUSY1 SISUSY1 SISUSY3 SISUSY4 NeSUSY2

AtSUSY1 OSSUSY 3 ZmSUSY1 SISUSY1

SISUSY3 SISUSY4 NeSUSY2

AtSUSY1 OSSUSY3 ZmSUSY1 SISUSY1 SISUSY3 SISUSY4 NeSUSY2

AtSUSY1 OsSUSY3 ZMSUSY1 SISUSY1 SISUSY3 SISUSY4 NeSUSY2
RVYDSEYCDILRVPFRTEKG-IVRKWISREEVWPYLETYTEDAAVELSKELNGKPDLII KVLGTEHTHILRVPFRTENG-TVRKWISRFEVWPYLETYTDDVAHEISGELQATPDLIIG KVLGTEHCHILRVPFRTENG-IVRKWISREEVWPYLETYTDDVAHEIAGELQANPDLI IG KVYGTEHSHILRVPFRTEKG-IVRKWISRFEVWPYMETFIEDVAKEISAELOAKPDLIIG KVFGTEHSHILRVPFRTEKG-IVRKWISRFEVWPYMETFIEDVGKEITAELQAKPDLIIG RISGTEYSHILRVPFRTENG-ILHKWISRFDVWPYLEKFTEDVAGEMSAELQGVPDLIIG KVSGCTNTWILRVPERKKNGEI IPQWISREEIWPHLETFALDVEREALAELGRRPDLIIG

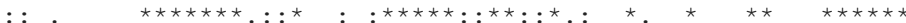
$\Delta$

GNLVASLLAHKLGVTQCTIAHALEKIKY PDSDIYWKKLDDKYHFSCOFTADIFAMN NYSDGNLVACLLAHKLGVTHCTIAHALEKTKYPNSDLYWKKFEDHYHFSCOFTADLIAMN NYSDGNLVACLLAHKMGVTHCTIAHALEKTKYPNSDLYWKKFEDHYHFSCQFTTDLIAMN NYSEGNLAASLLAHKLGVTQCTIAHALEKTKYPDSDIYWKKFDEKYHFSSQFTADLIAMN NYSEGNLAASLLAHKLGVTQCTIAHALEKTKYPDSDIYLNKFDEKYHFSAQFTADLIAMN NYSDGNLVASLLAYKMGITQCTIAHALEKTKYPDSDIYWKKFEEKYHFSCQFTADLLSMN NYSDGNLVATLLSRRLGVTQCNIAHALEKTKYLHSDIYWQENEDKYHFSCQYTADLLAMN

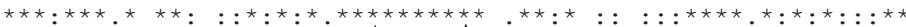
$\Delta$

$G T-B_{C}$

HTDFIITSTFQEIAGSKETVGQYESHTAFTLPGLYRVVHGIDVFDPKFNIVSPGADMSIY HADFIITSTFQEIAGNKETVGQYESHMAFTMPGLYRVVHGIDVFDPKFNIVSPGADMS IY HADFIITSTFQEIAGNKDTVGQYESHMAFTMPGLYRVVHGIDVFDPKFNIVSPGADLSIY HTDFIITSTFQEIAGSKDTVGQYESHMAFTMPGLYRVVHGINVFDPKFNIVSPGADINLY HTDFIITSTFOEIAGSKDTVGOYESHMAFTMPGLYRVVHGIDVFDPKFNIVSPGADVNLY HSDFIITSTYQEIAGTKNTVGQYESHTAFTLPGLYRVVHGIDVFDPKFNIVSPGADMTIY AADFIVTSTYQEIAGTREAEGQYESYRAFSMPGLYRVINGIDLFDPKFNIVSPGADAEVY

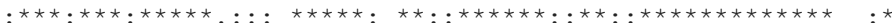

FPYTEEKRRLTKFHSE IEEL YSDVENKEHLCVLKDKKKP I LFTMARLDRVKN SGLVEW FPFTESQKRLTSLHLEIEELLFSDVENTEHKFVLKDKKKPI IFSMARLDHVKNLTGLVEL FPYTESHKRLTSLHPEIEELLYSOTENTEHKFVLNDRNKPI IFSMARLDRVKNLTGLVEL FPYSESEKRLTAFHPE IDELLYSDVENDEHLCVLKDRTKPILFTMARLDRVKNLTGLVEW FPYSEKEKRLTTFHPE IEDLLFSDVENEEHLCVLKDRNKPI I FTMARLDRVKNLTGLVEW FPYFDKEKRLTSLHPS IEKLLFDPEONEVHIGSLNDOSKPIIFSMARLDRVKNITGLVEC FPYTDQSRRLHSLIPE IESMLFDNTANFPARGILQDSDKPLIFTMARLDRIKNITGLVES

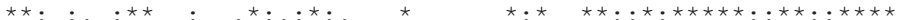

\section{$\Delta \boldsymbol{\Lambda}$}

YGKNTRLRELANLVVVGGDR-RKESKDNEEKAEMKKMYDLIEEYKLNGQFRWISSQMDRV 652 YGRNPRLQELVNLVVVCGDH-GKESKDKEEQAEFKKMFNLIEQYNLNGHIRWISAQMNRV 655 YGRNKRLOELVNLVVVCGDH-GNPSKDKEEOAEFKKMFDLIEOYNLNGHIRWISAOMNRV 655 YAKNPRLRGLVNLVVVGGDR-RKESKDLEEOAEMKKMYELIETHNLNGOFRWISSOMNRV 650 YAKNPRLRELVNLVVVGGDR-RKESKDLEEQAEMKKMYELIKTHNLNGQFRWISSQMNRV 650 YAKNATLRELANLVVVAGYNDVKKSNDREE IAEIEKMHALMKEHNLDGQFRWISAOMNRA $\quad 654$ YGASQRLRS LANLVIVGGKIDPQHSSDHEEQEQI HQMHHLMDEYKLDPQVRWLGMRLDKN 640

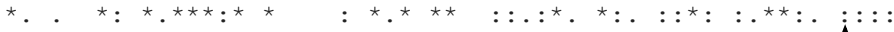

RNGEIYRYICDTKGAEVQPALYEAFGLTVVEAMTCGLPTEATCKGGPAEIIVHGKSGFHI RNGELYRYICDMRGAFVQPALYEAFGLTVIEAMTCGLPTFATAYGGPAEI IVHGVSGYH I RNGELYRYICDTKGAFVQPAFYEAFGLTVVEAMTCGLPTFATAYGGPAEI IVHGVSGYHI RNGELYRYIADTKGAFVQPAFYEAFGLTVVEAMTCGLPTFATNHGGPAEI IVHGKSGFHI RNGELYRYIADTRGAFVQPAFYEAFGLTVVEAMSCGLPTFATNQGGPAEIIVHGKSGFQI RNGELYRYIADKRGIFVQPAYYEAFGLTVVEAMTCGLPTFATCHGGPMEI IODGVSGYHI LAGELYRYIADKRGIFVQPALFEAFGLTIIEAMASGLPTFATRYGGPLEIIQHNRSGFH I

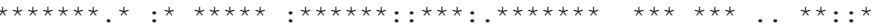


AtSUSY1

OsSUSY3

ZmSUSY1

SISUSY1

SISUSY3

SISUSY4

NeSUSY2

AtSUSY1

OsSUSY3

ZMSUSY1

SISUSY1

SISUSY3

SISUSY 4

NeSUSY2
DPYHGDQAADTLADFFTKCKEDPSHWDEISKGGLQRIEEKYTWQIYSQRLLTLTGVYGFW DPYQNDKASALLVEFFEKCQEDPNHWIKISQGGLQRIEEKYTWKLYSERLMTLSGVYGFW DPYQGDKASALLVDFFDKCQAEPSHWSKISQGGLQRIEEKYTWKLYSERLMTLTGVYGFW DPYHGEQAADLLADFFEKCKKEPSHWETISTGGLKRIQEKYTWQIYSERLLTLAAVYGFW DPYHGEQAADLLAEFFEKCKVDPSHWEAISKGGLKRIQEKYTWQIYSDRLLTLAAVYGFW DPYHPNKAAELMVEFFORCEQNPTHWENISASGLQRILDRYTWKIYSERLMTLAGVYGFW DPNQGTATADLIADFLEKSHEKPLEWERLSQGALARVASRYTWKLYAERMMTLSRIYSFW

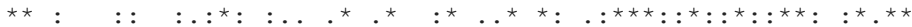

\section{S1 Table. Primers used for cloning}

\begin{tabular}{ll}
\hline Primer name & Primer sequence $\left(5^{\prime}-3^{\prime}\right)$ \\
\hline SUSY1_start_fw & ATGGCTGAACGTGTTCTGACTCG \\
SUSY1_end_rv & TCACTCAGCAGCCAATGGAACAG \\
SUSY1_BamHI_fw & CGCGGATCCATGGCTGAACGTGTTCTGACTCG \\
SUSY1_BamHI_rv & GCTGGATCCTCACTCAGCAGCCAATGGAACAG \\
SUSY3_start_fw & ATGGCTCAACGTGTTCTAACTCG \\
SUSY3_end_rv & TTACTCAACAGCCAATGGGACAA \\
SUSY3_BamHI_Fw & CGCGGATCCATGGCTCAACGTGTTCTAACTCG \\
SUSY3_BamHI_Rv & GATGGATCCTTACTCAACAGCCAATGGGACAA \\
SUSY4_start_fw & ATGTCGAATCCAAAGTTGTCAAG \\
SUSY4_end_rv & TCACTGCTTCTCATCAACTGCTA \\
SUSY4_BamHI_Fw & CGCATAGGATCCATGTCGAATCCAAAGTTGTCAAG \\
SUSY4_BamHI_Rv & GGCATCGGATCCTCACTGCTTCTCATCAACTGCTA \\
\hline
\end{tabular}


S2 Table. SUSY1 haplotypes and the corresponding tomato accessions

\begin{tabular}{|c|c|c|c|}
\hline$\#^{*}$ & Variations $* *$ & Accessions $* * *$ & \\
\hline 1 & none & $\begin{array}{ll}\text { S.lyc PI406760 } & \text { S.lyc LA1090 } \\
\text { S.lyc TR00003 } & \text { S.lyc LYC3306 } \\
\text { S.lyc V710029 } & \text { S.lyc EA01088 } \\
\text { S.lyc LYC1410 } & \text { S.lyc EA00940 } \\
\text { S.lyc TR00022 } & \text { S.lyc EA01640 }\end{array}$ & $\begin{array}{l}\text { S.lyc EA00488 } \\
\text { S.lyc EA00157 } \\
\text { S.lyc PI203232 } \\
\text { S.lyc TR00019 }\end{array}$ \\
\hline 2 & A709S & S.lyc EA03222 & \\
\hline 3 & R11S & $\begin{array}{ll}\text { S.lyc EA01155 } & \text { S.lyc PI272654 } \\
\text { S.lyc LA1324 } & \text { S.lyc TR00018 }\end{array}$ & S.lyc EA02054 \\
\hline 4 & V354G & $\begin{array}{ll}\text { S.lyc LA2706 } & \text { S.lyc LA2838A } \\
\text { S.lyc LA2463 } & \text { S.lyc LYC1969 } \\
\text { S.lyc LYC3476 } & \text { S.lyc LYC1343 } \\
\text { S.lyc LYC3153 } & \text { S.lyc EA00990 } \\
\text { S.lyc LA4451 } & \text { S.lyc PC11029 } \\
\text { S.lyc SG16 } & \text { S.lyc PI169588 } \\
\text { S.lyc TR00020 } & \text { S.lyc EA01037 } \\
\text { S.lyc TR00023 } & \text { S.lyc LYC2910 }\end{array}$ & $\begin{array}{l}\text { S.lyc EA00371 } \\
\text { S.lyc LYC1738 } \\
\text { S.lyc EA01049 } \\
\text { S.lyc PI303721 } \\
\text { S.lyc PI93302 } \\
\text { S.lyc EA01019 } \\
\text { S.lyc TR00021 }\end{array}$ \\
\hline 5 & R11S, V354G & $\begin{array}{l}\text { S.lyc PI311117 S.lyc PI158760 } \\
\text { S.lyc CGN15820 }\end{array}$ & S.lyc LA4133 \\
\hline 6 & R11S, S537T & $\begin{array}{ll}\text { S.lyc PI129097 } & \text { S.lyc LA0113 } \\
\text { S.pim LA1584 } & \text { S.hua LA1365 } \\
\text { S.hab LA1718 } & \text { S.pen LA1272 }\end{array}$ & $\begin{array}{l}\text { S.pim LYC2740 } \\
\text { S.cor LA0118 }\end{array}$ \\
\hline 7 & R11S, V354G, S537T & $\begin{array}{ll}\text { S.lyc EA00325 } & \text { S.lyc EA00375 } \\
\text { S.lyc LA1421 } & \text { S.lyc LA1479 } \\
\text { S.pim LYC2798 } & \text { S.pim LA1578 } \\
\text { S.neo LA0735 } & \text { S.arc LA2172 }\end{array}$ & $\begin{array}{l}\text { S.lyc LYC2962 } \\
\text { S.chm LA2663 } \\
\text { S.neo LA2133 } \\
\text { S.hab LA1777 }\end{array}$ \\
\hline 8 & R11S, S537T, R641K & S.chm LA2695 & \\
\hline 9 & R11S, S537T, E727D & S.chi CGN15532 & \\
\hline 10 & R11S, R375H, S537T & S.hab PI134418 & \\
\hline 11 & R11S, S537T, G600E, E727D & S.hua LA1364 & \\
\hline 12 & R11S, S537T, T569S, K730N & S.che LA0483 & \\
\hline 13 & R11S, I216V, V354G & S.hab LA0407 S.hab LYC4 & S.hab CGN157592 \\
\hline 14 & R11S, I216V, V354G, S537T & S.hab CGN157591 & \\
\hline 15 & R11S, A176T, V354G, S537T & S.arc LA2157 & \\
\hline 16 & R11S, A176T, S537T, T569S & S.arc LA385 & \\
\hline 17 & R11S, S537T, T569S, K730N & S.gal LA1044 & \\
\hline 18 & R11S, A176T, S537T, T569S, G600E & S.hua LA1983 & \\
\hline 19 & R11S, L318F, S537T, N635K & S.pen LA0716 & \\
\hline 20 & R11S, V354G, S537T, T569S, K730N & S.che LA1401 & \\
\hline 21 & E3D, R11S, G348S, S537T & S.per LA1278 & \\
\hline 22 & R11S, G348S, V354G, S537T & S.chi CGN15530 & \\
\hline 23 & $\begin{array}{l}\text { R11S, S73T, G348S, V354G, S537T, } \\
\text { K566M, E727D }\end{array}$ & S.per LA1954 & \\
\hline
\end{tabular}

*: haplotype number; ** x\#y: $\mathrm{x}$, amino acid of the reference Heinz cultivar; \#, amino acid position; $\mathrm{y}$, amino acid of the variant; *** S, Solanum; lyc, lycopersicum; cor, corneliomulleri; pim, pimpinellifolium; neo, neorickii; hua, huaylasense; hab, habrochaites; pen, pennellii; chm, chiemliewskii; chi, chilense; arc, arcanum; che, cheesmaniae; gal, galapagense. The accessions without bold highlight are listed as (old) cultivars and landraces, whereas the bold highlighted ones are considered as wild species. 
S3 Table. SUSY3 haplotypes and the corresponding tomato accessions

\begin{tabular}{|c|c|c|}
\hline$\#^{*}$ & Variations $* *$ & Accessions $* * *$ \\
\hline 1 & none & $\begin{array}{lll}\text { S.lyc LA2706 } & \text { S.lyc LA2838A } & \text { S.lyc PI406760 } \\
\text { S.lyc LA1090 } & \text { S.lyc EA00325 } & \text { S.lyc EA00488 } \\
\text { S.lyc EA00375 } & \text { S.lyc EA00371 } & \text { S.lyc LA2463 } \\
\text { S.lyc LYC1969 } & \text { S.lyc LYC1738 } & \text { S.lyc LYC3476 } \\
\text { S.lyc TR00003 } & \text { S.lyc EA01155 } & \text { S.lyc EA01049 } \\
\text { S.lyc LYC3153 } & \text { S.lyc EA03222 } & \text { S.lyc PI129097 } \\
\text { S.lyc PI272654 } & \text { S.lyc EA00990 } & \text { S.lyc EA00157 } \\
\text { S.lyc EA02054 } & \text { S.lyc PI303721 } & \text { S.lyc LA4451 } \\
\text { S.lyc V710029 } & \text { S.lyc PC11029 } & \text { S.lyc PI93302 } \\
\text { S.lyc SG16 } & \text { S.lyc EA01088 } & \text { S.lyc PI203232 } \\
\text { S.lyc PI311117 } & \text { S.lyc LA1324 } & \text { S.lyc PI158760 } \\
\text { S.lyc LYC1410 } & \text { S.lyc PI169588 } & \text { S.lyc TR00018 } \\
\text { S.lyc EA00940 } & \text { S.lyc TR00019 } & \text { S.lyc EA01019 } \\
\text { S.lyc TR00020 } & \text { S.lyc EA01037 } & \text { S.lyc TR00021 } \\
\text { S.lyc TR00022 } & \text { S.lyc TR00023 } & \text { S.lyc EA01640 } \\
\text { S.lyc LA4133 } & \text { S.pim LYC2740 } & \text { S.lyc LYC2910 }\end{array}$ \\
\hline 2 & D555Y & S.che LA1401 \\
\hline 3 & S106I & S.che LA0483 S.gal LA1044 \\
\hline 4 & S106I, K741E & $\begin{array}{lll}\text { S.lyc LYC1343 } & \text { S.lyc LYC3306 } & \text { S.lyc LA0113 } \\
\text { S.lyc LYC2962 } & \text { S.lyc LA1421 } & \text { S.lyc LA1479 } \\
\text { S.pim LYC2798 } & \text { S.lyc CGN15820 }\end{array}$ \\
\hline 5 & S106I, H361R, K741E & S.pim LA1584 \\
\hline 6 & S106I, D555Y, K741E & S.pim LA1578 \\
\hline 7 & S106I, E724D, K741E & S.hab LA1718 \\
\hline 8 & S106I, Q199H, E724D, K741E & $\begin{array}{ll}\text { S.hab CGN157591 } & \text { S.hab CGN157592 } \\
\text { S.hab LA1777 } & \text { S.hab LA0407 }\end{array}$ \\
\hline 9 & S53A, S106I, E727D, K741E & S.arc LA385 $\quad$ S.arc LA2172 \\
\hline 10 & S53A, S106I, Q349L, E727D, K741E & $\begin{array}{l}\text { S.chm LA2663 } \\
\text { S.neo LA2133 }\end{array}$ \\
\hline 11 & S53A, S106I, E559D, E727D, K741E & S.arc LA2157 \\
\hline 12 & $\begin{array}{l}\text { S106I, N188K, Q199L, E724D, E727D, } \\
\text { K741E }\end{array}$ & S.cor LA0118 \\
\hline 13 & S73C, S106I, N188K, E724D, E727D, K741E & S.per LA1278 \\
\hline 14 & S73C, S106I, N188K, E724D, K741E & S.per LA1954 \\
\hline 15 & I88F, S106I, E724D, K741E & S.hab PI134418 \\
\hline 16 & S106I, P546S, E629G, E724D, K741E & S.pen LA0716 \\
\hline 17 & S53A, S106I, E724D, E727D, K741E & S.pen LA1272 \\
\hline 18 & S106I, E724D, E727D, K741E, E798K & S.hua LA1983 \\
\hline 19 & $\begin{array}{l}\text { S106I, N188K, W591G, E724D, E727D, } \\
\text { K741E, E798K }\end{array}$ & S.chi CGN15530 \\
\hline 20 & $\begin{array}{l}\text { S106I, N188K, Q199H, S219P, E724D, } \\
\text { E727D, K741E, E798K }\end{array}$ & S.hua LA1365 \\
\hline 21 & $\begin{array}{l}\text { S106I, N188K, H634Y, E724D, E727D, } \\
\text { K741E, E798K }\end{array}$ & S.chi CGN15532 \\
\hline 22 & $\begin{array}{l}\text { S53A, S106I, N188K, Q199H, S219P, E724D, } \\
\text { E727D, K741E, E798K }\end{array}$ & S.hua LA1364 \\
\hline
\end{tabular}

*: haplotype number; $* * \mathrm{x} \# \mathrm{y}: \mathrm{x}$, amino acid of the reference Heinz cultivar; \#, amino acid position; $\mathrm{y}$, amino acid of the variant; *** S, Solanum; lyc, lycopersicum; cor, corneliomulleri; pim, pimpinellifolium; neo, neorickii; hua, huaylasense; hab, habrochaites; pen, pennellii; chm, chiemliewskii; chi, chilense; arc, arcanum; che, cheesmaniae; gal, galapagense. The accessions without bold highlight are listed as (old) cultivars and landraces, whereas the bold highlighted ones are considered as wild species. 
S4 Table. SUSY4 haplotypes and the corresponding tomato accessions

\begin{tabular}{|c|c|c|c|c|}
\hline \#* & Variations* & Accessions $* *$ & & \\
\hline \multirow{18}{*}{1} & \multirow{18}{*}{ None } & S.lyc LA2706 & S.lyc LA2838A & S.lyc PI406760 \\
\hline & & S.lyc LA1090 & S.lyc EA00325 & S.lyc EA00488 \\
\hline & & S.lyc EA00375 & S.lyc EA00371 & S.lyc LA2463 \\
\hline & & S.lyc LYC1969 & S.lyc LYC1738 & S.lyc LYC3476 \\
\hline & & S.lyc TR00003 & S.lyc LYC1343 & S.lyc LYC3306 \\
\hline & & S.lyc EA01155 & S.lyc EA01049 & S.lyc LYC3153 \\
\hline & & S.lyc EA03222 & S.lyc PI129097 & S.lyc PI272654 \\
\hline & & S.lyc EA00990 & S.lyc EA00157 & S.lyc EA02054 \\
\hline & & S.lyc PI303721 & S.lyc LA4451 & S.lyc V710029 \\
\hline & & S.lyc PC11029 & S.lyc PI93302 & S.lyc SG16 \\
\hline & & S.lyc EA01088 & S.lyc PI203232 & S.lyc PI311117 \\
\hline & & S.lyc LA1324 & S.lyc PI158760 & S.lyc LA0113 \\
\hline & & S.lyc LYC1410 & S.lyc PI169588 & S.lyc TR00018 \\
\hline & & S.lyc EA00940 & S.lyc TR00019 & S.lyc EA01019 \\
\hline & & S.lyc TR00020 & S.lyc EA01037 & S.lyc TR00022 \\
\hline & & S.lyc EA01640 & S.lyc LA4133 & S.lyc LA1421 \\
\hline & & S.pim LYC2740 & S.che LA1401 & S.lyc LYC2910 \\
\hline & & $\begin{array}{l}\text { S.pim LYC2798 } \\
\text { S.gal LA1044 }\end{array}$ & S.che LA0483 & S.pim LA1578 \\
\hline 2 & V35G & $\begin{array}{l}\text { S.lyc LYC2962 } \\
\text { S.pim LA1584 }\end{array}$ & $\begin{array}{l}\text { S.lyc TR00021 } \\
\text { S.lyc CGN15820 }\end{array}$ & S.lyc LA1479 \\
\hline 3 & V408G, V585G & S.lyc TR00023 & & \\
\hline 4 & V114I & $\begin{array}{l}\text { S.arc LA385 } \\
\text { S.chi CGN15532 }\end{array}$ & \multicolumn{2}{|c|}{ S.chi CGN15530 } \\
\hline 5 & V114I, N212S & S.hua LA1365 & \multicolumn{2}{|l|}{ S.pen LA1272 } \\
\hline 6 & V114I, D229E & S.hab LA1777 & & \\
\hline 7 & V114I, N175S & S.chm LA2663 & \multicolumn{2}{|l|}{ S.chm LA2695 } \\
\hline 8 & V114I, S567N & S.arc LA2157 & & \\
\hline 9 & V35G, V114I, N212S & S.per LA1278 & \multicolumn{2}{|l|}{ S.hua LA1364 } \\
\hline 10 & V35G, V114I, N175S, T738A & S.neo LA2133 & \multicolumn{2}{|l|}{ S.neo LA0735 } \\
\hline 11 & V114I, N175S, S567N & S.arc LA2172 & & \\
\hline 12 & V114I, D229E, S567N & S.hab PI134418 & \multicolumn{2}{|l|}{ S.hab LA1718 } \\
\hline 13 & V114I, D229E, S567N, R732Q & \multicolumn{3}{|c|}{ S.hab CGN157591 S.hab LYC4 } \\
\hline 14 & V114I, D229E, S567N, T688I & \multicolumn{3}{|c|}{ S.hab CGN157592 } \\
\hline 15 & V114I, D229E, S567N, V683L, T688I & \multicolumn{3}{|l|}{ S.hab LA0407 } \\
\hline 16 & V114I, N212S, N281S, L634F & \multicolumn{3}{|l|}{ S.cor LA0118 } \\
\hline 17 & V35G, V114I, N175K, K325N, S567N & \multicolumn{3}{|l|}{ S.pen LA0716 } \\
\hline
\end{tabular}

*: haplotype number; ** x\#y: x, amino acid of the reference Heinz cultivar; \#, amino acid position; $\mathrm{y}$, amino acid of the variant; *** S, Solanum; lyc, lycopersicum; cor, corneliomulleri; pim, pimpinellifolium; neo, neorickii; hua, huaylasense; hab, habrochaites; pen, pennellii; chm, chiemliewskii; chi, chilense; arc, arcanum; che, cheesmaniae; gal, galapagense. The accessions without bold highlight are listed as (old) cultivars and landraces, whereas the bold highlighted ones are considered as wild species. 


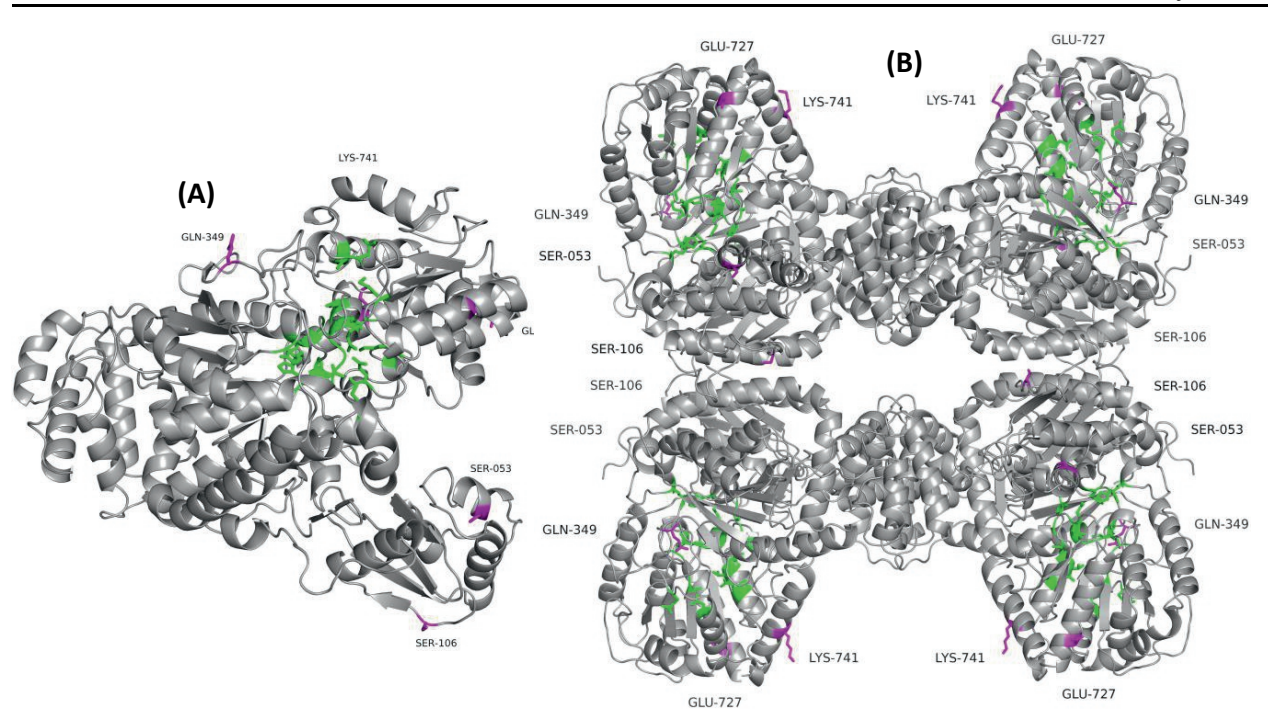

S1 Fig. Ribbon drawings of the 3D modelled tomato SuSy3 haplotype\#1 monomer (A) and homo-tetramer (B) based on SuSy1 of $\boldsymbol{A}$. thaliana (PDB ID 3S28). The amino acid residues interacting with substrate are indicated in green, while the residues S53, S106, Q349, E727, and K741 with variation in SuSy3-haplotype\#9 and SuSy3-haplotype\#10 are highlighted in magenta. All these residues with amino acid change are not close to the binding sites, or in the interfaces between the subunits.
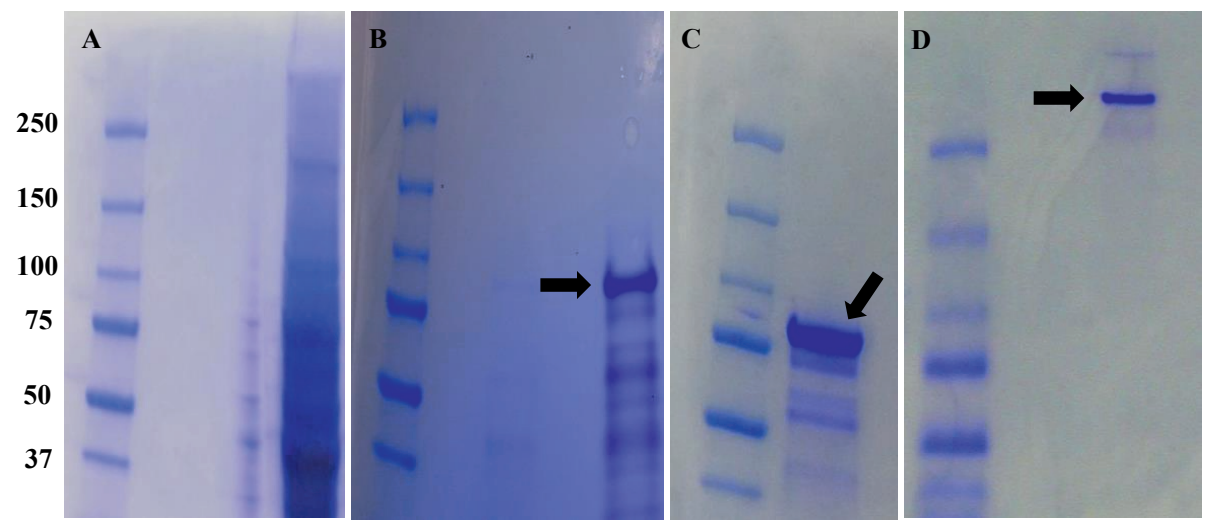

S2 Fig. Purification of the recombinant enzyme SuSy3-haplotype\#1 (cv. Moneymaker) according to Römer et al. 2004. In the crude extract step, no specific band of expected monomer SUSY product of $92 \mathrm{kDa}$ was observed in the SDS-PAGE gel (A). Once the crude extract has been purified by Ion-exchange chromatography (IEX), the expected band of approximately $90 \mathrm{kDa}$ product (black arrow) became visible on SDS-PAGE gel (B). This IEX purified product went through the Immobilized metal ion affinity chromatography (IMAC) and became clearer (black arrow) with fewer unspecific band (C). The IMAC purified product was loaded on a native-PAGE and seen as the expected tetramer product (black arrow) (D). The protein ladder Precision Plus Protein ${ }^{\mathrm{TM}}$ Prestained Standards (BioRad, USA) was used in all the PAGE gels above. 


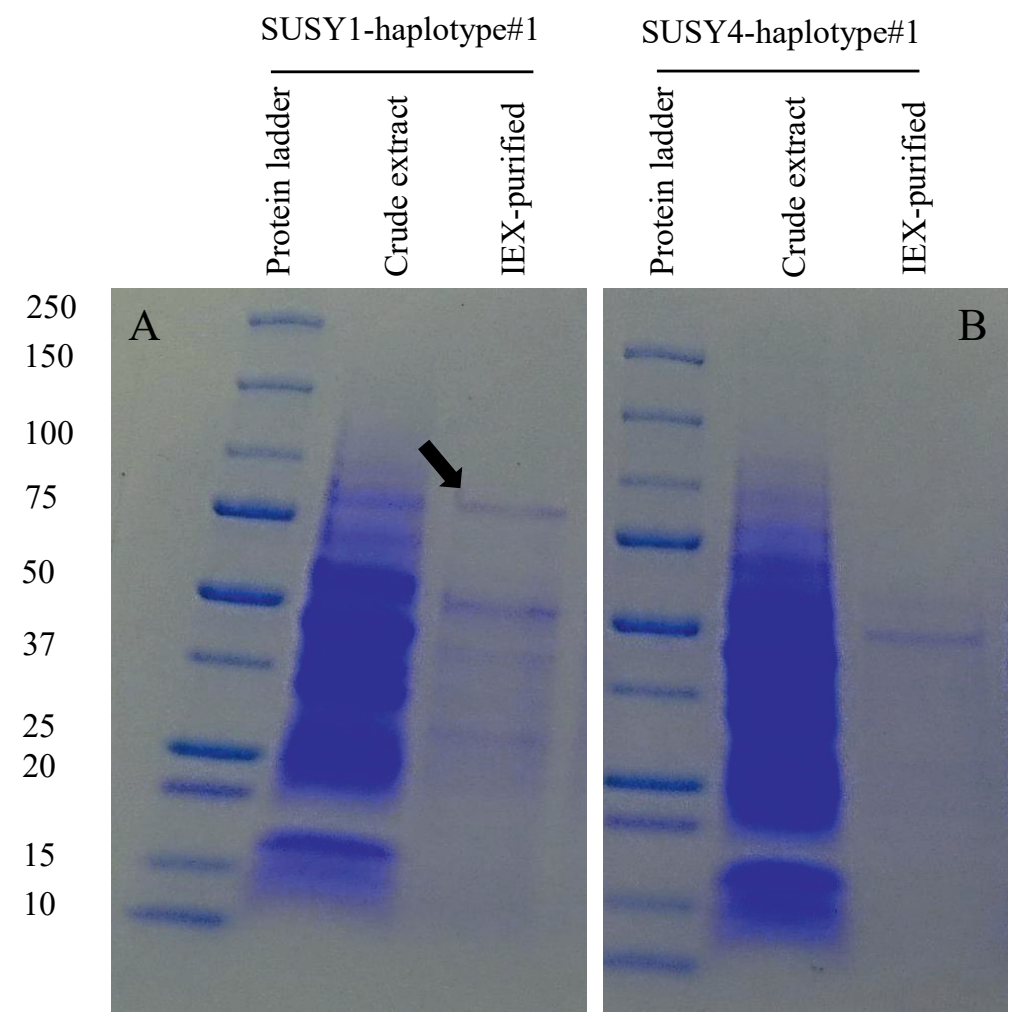

S3 Fig. SDS-PAGE gels of the recombinant enzymes SuSy1-haplotype\#1 and SuSy4haplotype\#1 (cv. Moneymaker) purified by Ion-exchange chromatography (IEX) according to Römer et al. 2004. An enhancement of the expected monomer SuSy1-haplotype\#1 product of $92 \mathrm{kDa}$ (black arrow) was observed in the IEX purified sample compared to the crude extract (A). However, the enhancement of IEX-purified SuSy1-haplotype\#1 was less intense when compared to that in the IEX-purified SuSy3-haplotype\#1 shown in Fig. S1. For SuSy4, the expected product of $92 \mathrm{kDa}$ was not found in SDS-PAGE after the purification with IEX (B). The protein ladder Precision Plus Protein ${ }^{\mathrm{TM}}$ Prestained Standards (BioRad, USA) was used in all the SDS-PAGE gels above. 


\section{Chapter 4}

\section{Introgression of a region harboring a sucrose synthase allele from a wild relative into cultivated tomato increases dry matter allocation to fruits}

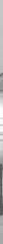

Whit ath 4

Quy Dung Dinh ${ }^{1,2^{*}}$, Anne J. Kortstee ${ }^{1,3^{*}}$, Richard G. F. Visser ${ }^{1}$, Luisa M.

Trindade $^{1}$

${ }^{1}$ Plant Breeding, Wageningen University and Research, P.O. Box 386, $6700 \mathrm{AJ}$ Wageningen, The Netherlands

2. Graduate School Experimental Plant Sciences, Wageningen University, Wageningen, The Netherlands

${ }^{3}$ Current address: CTGB Board for authorisation of plant protection products and biocides, Ede, The Netherlands

*These authors contributed equally to this work. 


\begin{abstract}
Sucrose synthase directly hydrolyses sucrose and has been suggested to be a biochemical marker for plant sink strength. Sink strength, in turn, plays an important role in plant growth and development. This study describes the effects of the introgression of a sucrose synthase gene (Susy3) from a high altitude wild accession Solanum neorickii G1.1601 into a cultivated tomato Moneymaker background (Susy3-IL) on growth, biomass allocation, and carbohydrate metabolism. The results were compared to counterparts of the recurrent parental background at control growth temperature $\left(\mathrm{CT}, 25 / 20^{\circ} \mathrm{C}\right.$ day/night) and suboptimal growth temperature (SOT, $16 / 14^{\circ} \mathrm{C}$ day night). Overall, Susy3-IL showed an inferior performance relatively to cv. Moneymaker for several agronomic traits such as total leaf area, total dry weight production, and fruit dry weight at both temperatures. However, Susy3-IL had a 9\% increase in dry mater (DM) allocation to fruits compared to that in cv. Moneymaker during the fruit ripening phase at CT. This improvement was caused by a higher accumulation of total soluble sugars and starch in Susy3-IL fruits and a decrease in total soluble sugars in its leaves. Furthermore, the ripe fruits of Susy3-IL had higher expression levels of sucrose synthase encoding genes Susy1/3/4 and cell wall invertase encoding genes Lin5 than cv. Moneymaker background's counterparts. However, that trend was not observed in their enzyme activities. These preliminary results indicate that wild Susy3 alleles might not be the only and direct key player contributing to the increase in DM allocation in sink fruits. The allele(s) responsible for this improvement in DM allocation are still to be identified. Nevertheless these results strengthen the potential of wild species germplasm as the source for improving sink strength and plant performance in cultivated tomatoes.
\end{abstract}




\section{Introduction}

Yield is an important trait for tomato breeding. Factors determining tomato yield are total biomass production, biomass partitioning and fruit dry matter content. Among those factors, biomass partitioning and fruit dry matter content are more crucial because they will affect directly the yield and quality of tomato fruits (Heuvelink and Dorais 2005). Biomass production is mainly driven by photosynthesis, which takes place in source leaves and results in the synthesis of sugars, further accumulated as sucrose. Sucrose is subsequently transported from the source leaves to different sink organs such as developing leaves, roots, flowers and fruits. Sucrose partitioning among the sink organs depend on sink strength which is defined as the competitive ability of an organ to accumulate photoassimilates (Marcelis 1996). Thus sucrose metabolism in sink organs plays an important role in determining plant sink strength and the enzymes involved in sucrose metabolism would be potential candidates to enhance sink strength.

Sucrose synthase (SUSY) is one of the two classes of enzymes known to directly hydrolyse sucrose, of which several isoforms are present in most plant tissues at any given time (Ruan 2014). Three isoforms SUSY1/3/4 have been identified in tomato, are encoded by the genes Susyl, Susy3 and Susy4, and mapped on chromosome 12, 7 and 9, respectively (Wang et al. 1993b, Chengappa 1998, Goren et al. 2011). SUSY has been suggested as a biochemical marker for sink strength in tomato for several reasons. These include: (1) energy efficiency in sucrose breakdown to directly generate hexose phosphates for further metabolism (Xu et al. 1989); (2) strong correlation between SUSY activity, ADP-glucose pyrophosphorylase, and starch accumulation in young fruits (Robinson et al. 1988, Yelle et al. 1988); (3) positive correlation between SUSY activity and fruit growth and final fruit size in both domesticated and wild species tomatoes (Sun et al. 1992); (4) an important role exists for SUSY in sucrose unloading capacity and fruit setting in young tomatoes (D'Aoust et al. 1999). Therefore it is of interest to enhance the sucrose-hydrolyzing capacity of SUSY in cultivated tomato, which in turn might help to improve plant sink strength.

Improving plant sink strength at sub-optimal growth temperature is also essential when we want to reduce the energy consumption while growing plants such as tomatoes in heated glasshouses, which is especially relevant for countries with a cool-temperate or cold climate. It has been shown that carbohydrate metabolism is altered when plants are exposed to cold stress (reviewed by Ma et al. 2009). Kaur et al. (2009) showed that the SUSY activity was significantly higher in seeds of several cold tolerant chickpea lines in comparison to the cold susceptible ones. In addition, Kortstee et al. (2007) reported that high altitude wild species tomato like S. arcanum LA385 and S. habrochaites LA1777 have different regulation patterns in genes and enzymes involved in carbohydrate metabolism such as SUSY, which leads to distinct carbohydrate compositions in their fruits compared to the cultivated tomato. Despite the fact that the important role of SUSY in plant sink strength has been shown with overexpression and down-regulation of gene expression by RNA-induced gene silencing experiments as mentioned above, it has never been investigated via the introgression of Susy allele(s) from a wild species into a cultivated tomato background. Therefore in this study we examined the effects of the introgression of the single allele Susy3 from the high altitude wild 
species accession $S$. neorickii G1.1601 in a cultivated tomato background on plant growth, biomass allocation and regulation of sucrose metabolism at transcript and protein levels. These effects were assessed at two growth temperature regimes: control temperature of $25^{\circ} \mathrm{C}$ (CT) and sub-optimal temperature of $16^{\circ} \mathrm{C}$ (SOT).

\section{Materials and methods}

\section{Plant materials}

Two genotypes were used in this study: cultivated tomato Solanum lycopersicum cv. Moneymaker and an introgression line containing wild allele Susy3 called Susy3-IL. This introgression line was selected from the BC3S2 population between cv. Moneymaker and wild species S. neorickii G1.1601 (also known as LA0735) (Finkers et al. 2008). This wild species was collected in 1959 in Huariaca - Peru at an elevation of 2900 m.a.s.l., and has an autogamous self-compatibility mating system (UC Davis Tomato Genetics Resource Center). Supplementary data 1 shows how the Susy3-IL was identified from the BC3S2 population (Fig. S1).

\section{Growth conditions and treatments}

In the glasshouse, seeds of cv. Moneymaker and Susy3-IL were germinated and seedlings were grown into plants. When they started to flower (approximately after eight weeks), the plants were moved into two different climate chambers. Both climate chambers had 14/10 h day/night regime, irradiance of $250-300 \mu \mathrm{mol}$ photons $\mathrm{m}^{-2} \mathrm{~s}^{-1}$ at plant level during the day, and average relative humidity of $70-75 \%$. The only difference between the two climate chambers was the temperature regime, in one it was set at $25 / 20^{\circ} \mathrm{C}$ day/night temperature for control temperature (CT), and in the other it was set at $16 / 14{ }^{\circ} \mathrm{C}$ day/night for sub-optimal temperature (SOT). Plants were given nutrients weekly during the first five weeks, and two to three times per week from the sixth week onward. In addition, side shoots were removed regularly.

\section{Analysis of growth and development}

As indicated above both, genotypes started to flower in week 8 and form fruits in week 10 . Different plant characteristics were measured every two weeks. Those characteristics were plant height, total leaf area, number of fruits, fresh weight and dry weight of leaves, stems, fruits, and roots. These measurements were performed on a single plant for each genotype at each time point. Total leaf area was measured by a leaf area meter (model 3100, Li-Cor Inc., Lincoln, NE, USA). Relative leaf thickness was calculated as the division of the total leaf area by leaf fresh weight (Medek et al. 2007). Specific leaf area is calculated by division of the total leaf area by leaf dried weight. After fresh weight was measured, a small portion of each tissue was collected, snap-frozen in liquid nitrogen and stored at $-80^{\circ} \mathrm{C}$ for RNA isolation, protein extraction, and metabolite measurements till further use. 


\section{Chlorophyll fluorescence measurement}

Chlorophyll fluorescence was measured using the Rapid Plant Stress Screening Device model OS-30p (ADC BioScientific Ltd, UK). In this work, minimum fluorescence $\left(\mathrm{F}_{\mathrm{O}}\right)$ and maximum fluorescence $\left(F_{M}\right)$ were determined with 20-min dark adapted leaves. Subsequently, the maximum quantum efficiency of photosystem II (PSII) photochemistry was calculated with the following equation $F_{V} / F_{M}=\left(F_{M}-F_{O}\right) / F_{M}$.

\section{Soluble sugar and starch content measurements}

Twenty milligrams of ground plant tissues were used for extraction and measurement of soluble sugars and starch content. The extraction was performed as described in Kortstee et al. (2007). Soluble sugars (sucrose, fructose, and glucose) and starch were measured with kits No. 10716260035 and No. 10207748035 (R-Biopharm, Germany), respectively. The measurements were carried out according to the manufacturer's instructions, but with 10-fold scaled down in liquid volume for microtiter plates readings.

\section{Extraction and activity determination of enzymes}

From each genotype, extracts for enzyme assays were prepared according to the protocols described in Appeldoorn et al. (1997) with some modifications. Briefly, five milligrams of powdered freeze-dried fruits were homogenized in $500 \mu \mathrm{L}$ extraction buffer containing 50 mM Hepes-KOH (pH 7.5), 1 mM EDTA, 2mM of phenylmethanesulfonyl fluoride (PMSF), $5 \mathrm{mM}$ dithiothreitol (DTT) and 10\% (v/v) glycerol. After centrifugation for $5 \mathrm{~min}$ at $15,000 \mathrm{x}$ $\mathrm{g}$, the supernatant was collected, aliquoted, and stored at $-80 \circ \mathrm{C}$ until further use. The supernatant (i.e. extract) contained all soluble proteins including sucrose synthase, and vacuolar invertase, whereas the pellet was subsequently used to extract cell wall bound proteins such as cell wall invertase. The pellet was then resuspended in a washing buffer consisting of the same ingredients as the extraction buffer but without DTT. After centrifugation for $5 \mathrm{~min}$ at $13,000 \mathrm{rpm}$, the supernatant was discarded and the whole procedure was repeated three times. Afterward, pellets were incubated in 5001 of buffer containing 20mM 2-(N-morpholino) ethanesulphonic acid (Mes)-KOH (pH 6.0) and $1.0 \mathrm{M}$ $\mathrm{NaCl}$. The samples were left for $16 \mathrm{~h}$ in a shaker at $4{ }^{\circ} \mathrm{C}$ and subsequently centrifuged for 15 $\min$ at $13,000 \mathrm{rpm}$. Supernatant was then collected, aliquoted, and stored at $-80^{\circ} \mathrm{C}$ until further use. The whole extraction procedure was performed at $4{ }^{\circ} \mathrm{C}$ for both soluble extracts and cell wall extracts. Subsequently, all the activities of different enzymes were determined at two different temperatures, i.e. control $25^{\circ} \mathrm{C}$ and sub-optimal $16^{\circ} \mathrm{C}$.

\section{Sucrose synthase activity measurements}

Activity of sucrose synthase was assayed as in Appeldoorn et al. (1997) with some modifications. Briefly, $5 \mu \mathrm{L}$ of extract were incubated for $30 \mathrm{~min}$ at the desired temperature in $95 \mu \mathrm{L}$ of the assay medium containing 20mM Hepes- $\mathrm{KOH}$ (pH 7.5), $200 \mathrm{mM}$ sucrose, and $4 \mathrm{mM}$ UDP. The reaction was terminated by incubation at $95 \circ \mathrm{C}$ for $4 \mathrm{~min}$. The formation of UDP-glucose was assayed in $100 \mu \mathrm{L}$ of medium containing $0.4 \mathrm{M}$ glycine $\mathrm{pH} 8.9,10 \mathrm{mM}$ $\mathrm{MgCl}_{2}, 4 \mathrm{mM} \mathrm{NAD}{ }^{+}$, and adding $0.02 \mathrm{U}$ of UDP-glucose dehydrogenase to start the reaction. 
For the blanks, the reaction was terminated immediately after adding assay medium into extract by incubation at $95^{\circ} \mathrm{C}$ for $4 \mathrm{~min}$.

\section{Vacuolar invertase activity measurements}

Activity of vacuolar invertase was assayed as described in Appeldoorn et al. (1997) with small modifications. Ten $\mu \mathrm{L}$ of extract were incubated in $95 \mu \mathrm{L}$ of the assay medium containing $30 \mathrm{mM}$ citrate-phosphate buffer $\mathrm{pH} 5.2$ and $25 \mathrm{mM}$ sucrose for $60 \mathrm{~min}$ at $25^{\circ} \mathrm{C}$ or $16^{\circ} \mathrm{C}$. The formation of glucose was determined with D-glucose kit No. 10716251035 (RBiopharm, Germany) according to manufacturer's instructions, with 10- fold scale down for microtiter plate readings.

\section{Cell wall invertase activity measurements}

Activity of cell wall invertase was measured in a similar way as in vacuolar invertase activity, but instead of vacuolar assay buffer a cell wall assay buffer was used consisting of $25 \mathrm{mM}$ citrate-phosphate buffer $\mathrm{pH} 4.6$ and $25 \mathrm{mM}$ sucrose.

\section{Gene expression}

Expression levels of genes encoding sucrose synthases and invertases that are directly involved in the hydrolysis of sucrose were analyzed by qRT-PCR using the primers listed in Supplementary data 2 . Total RNA was prepared from about $2-5 \mathrm{mg}$ tissue ground in liquid nitrogen using standard CTAB mini-isolation protocol (Untergasser 2008). The isolated RNA was then treated with DNase I (Invitrogen, USA), and converted into cDNA with iScriptTM cDNA synthesis kit (BioRad, USA). iQ SYBR Green supermix (Bio-Rad, US) was used on the CFX96TM Real Time System C100TM Thermo Cycler (Bio-Rad, US). The program of the thermocycler was set at: denaturation for $10 \mathrm{~min}$ at $95^{\circ} \mathrm{C}$ followed by 40 cycles of $15 \mathrm{~s}$ at $95^{\circ} \mathrm{C}$ and $1 \mathrm{~min}$ annealing and extension at $60^{\circ} \mathrm{C}$. In addition, melting curve analysis was performed to assess whether a single specific PCR product was formed by each primer pair combination, in which the thermocycler program consisted of sixty-one cycles of $60^{\circ} \mathrm{C}+$ $0.5^{\circ} \mathrm{C}$. Subsequently relative quantification of the target gene expression levels were calculated using the Act gene as the reference gene, employing the $2^{-\Delta \Delta \mathrm{Ct}}$ method.

\section{Statistical analysis}

One-way analysis of variance (ANOVA) was used to evaluate statistically significant effects of temperature on different parameters. Each parameter was measured in three to five individual plants. Statistical analyses were carried out with GenStat $17^{\text {th }}$ edition (VSN International Ltd, UK). 


\section{Results}

\section{Chlorophyll fluorescence maintained at both temperatures}

The maximum quantum efficiency of photosystem II $\left(\mathrm{F}_{\mathrm{v}} / \mathrm{F}_{\mathrm{m}}\right)$ was measured in both the introgression line Susy3-IL and the recurrent parent cv. Moneymaker during their growth at two different temperature regimes, i.e. control temperature (CT) of $25^{\circ} \mathrm{C} / 20^{\circ} \mathrm{C}$ and suboptimal temperature (SOT) of $16^{\circ} \mathrm{C} / 14^{\circ} \mathrm{C}$ from flowering till fruit ripening. In this study, the $\mathrm{F}_{\mathrm{v}} / \mathrm{F}_{\mathrm{m}}$ value of both genotypes were between $0.80-0.81$ (Fig. 1). There was no consistent impact of temperature in both genotypes, except cv. Moneymaker grown at CT having lower $\mathrm{F}_{\mathrm{v}} / \mathrm{F}_{\mathrm{m}}$ value than those at SOT in week 12.
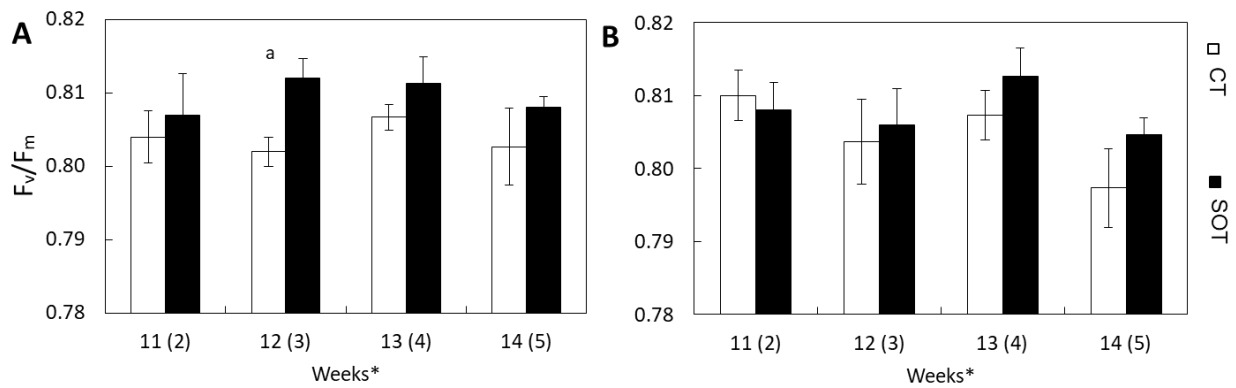

Figure 1. Maximum quantum efficiency of photosystem II photochemistry $\left(\mathrm{F}_{\mathrm{v}} / \mathrm{F}_{\mathrm{m}}\right)$ of cv. Moneymaker (A) and Susy3-IL (B) from week 11 till 14 after sowing. The number in parentheses refers to the number of weeks at SOT. The black and white columns are the values at CT and SOT, respectively. The error bars represent standard error of three individual plants $( \pm \mathrm{SE})$. Significant difference is denoted as a for $P<0.05$.

\section{Susy3-IL showed a decrease in growth and development}

Plant growth and development of Susy3-IL and its recurrent parent cv. Moneymaker at CT and SOT from onset of flowering till fruit ripening were studied by measuring several parameters such as total leaf area, total dry weight, number of fruits and fruit dry weight. SOT generally had a negative influence on those measured traits in both genotypes. Susy3-IL had a reduction in total leaf area, specific leaf area, total dry weight, number of fruits, and fruit dry weight in comparison to those of cv. Moneymaker (Fig. 2). In addition, the leaves in both genotypes became thicker at SOT, especially in older plants (Fig. 2D). The leaves of both genotypes became curlier at SOT compared to those at CT (data not shown). 

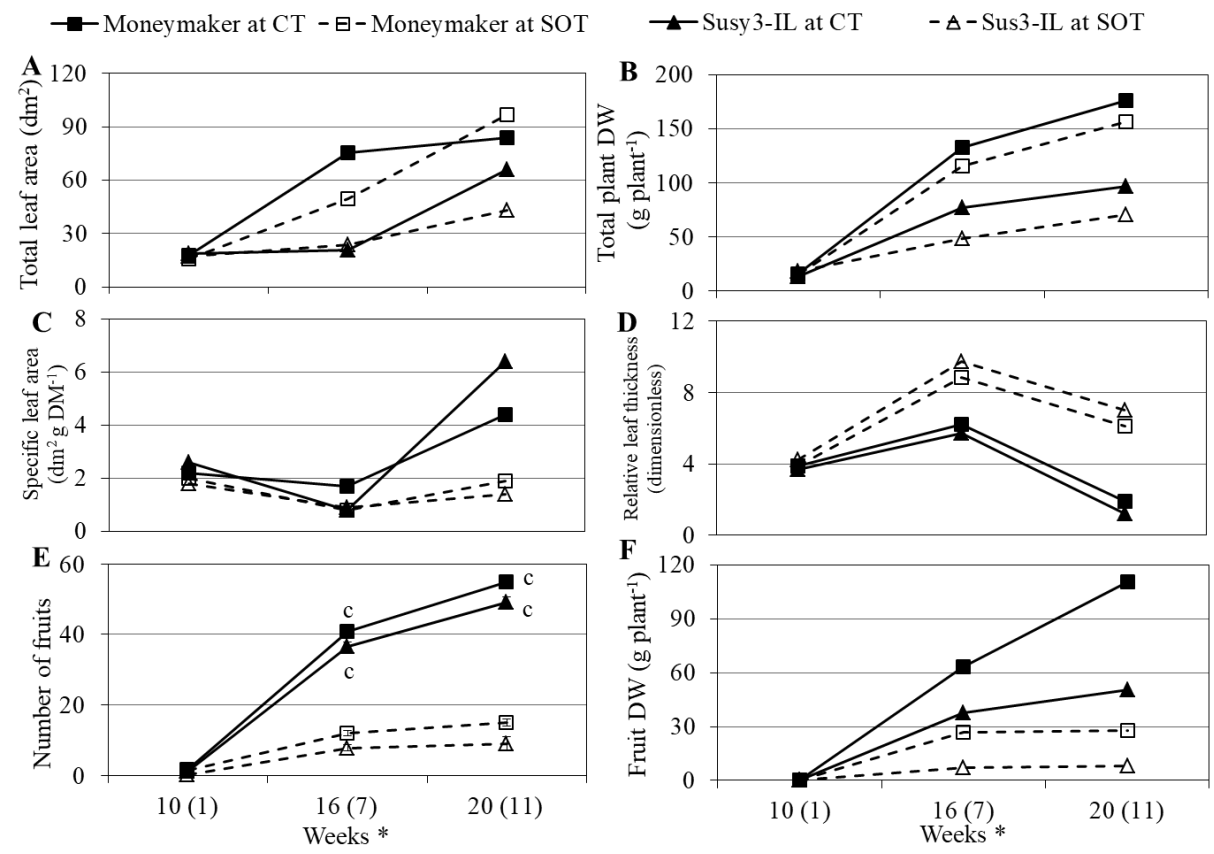

Figure 2. Measurements of several growth and development parameters. These parameters are total leaf area (A), Total plant DW (B), specific leaf area (C), relative leaf thickness (D), number of fruits $(\mathrm{E})$, and fruit dry weight $(\mathrm{F})$, which were measured in week 10, 16, and 20. The number in parentheses refers to the number of weeks at SOT. Data in panels A-D and F are derived from measurement on one single plant, whereas data in panel E represent the mean of three to five individual plants $( \pm \mathrm{SE})$. Significant difference between samples of the same genotype but at different growth temperatures is denoted as "c" for $P<0.001$ in panel E.

\section{Susy3-IL shows an increase in dry matter allocation to fruits at CT}

Dry matter (DM) allocation to different organs of both Susy3-IL and cv. Moneymaker at CT and SOT was measured on plants with young fruits till ripe fruits. The results showed that developmental stages and temperatures had similar effect on DM allocation to different organs of both plant genotypes (Fig. 3). DM allocation to leaves and roots decreased, whereas more DM was accumulated in stems and especially fruits as plants grew older. Concerning growth temperatures, SOT increased the DM fraction to leaves and roots (Fig. 3A, C), but decreased the fraction to fruits (Fig. 3D). However the DM fraction to stems did not appear to be affected by temperature (Fig. 3B). Focusing on fruits as the marketable product, we observed a $9 \%$ increase in DM fraction to fruits of Susy3-IL (58\%) compared to that of cv. Moneymaker (49\%) in week 20 at CT (Fig. 3D, see also Supplementary data 3). But at SOT in the same week this fraction to fruits of Susy3-IL (10\%) was twofold lower than that of cv. Moneymaker (21\%). 

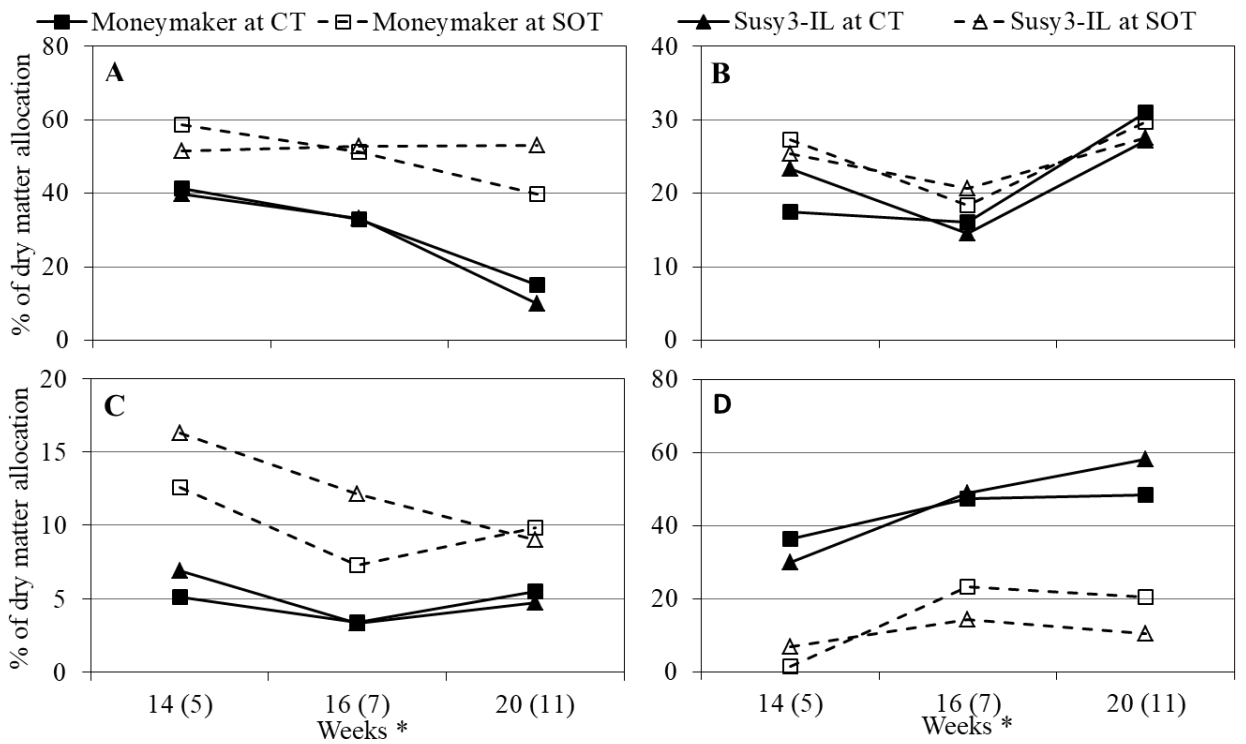

Figure 3. Dry matter allocation to leaves (A), stems (B), roots (C), and fruits (D) in relation to total plant dry weight at week 14,16 , and 20 . The number in the parentheses is number of weeks that plants were grown at SOT. The data in these panels are derived from measurement on one single plant.

\section{Different profiles of soluble sugars and starch contents found in distinct organs and at different growth temperatures}

The primary products of carbohydrate metabolism, i.e. soluble sugars and starch, were measured in leaves and green fruits of week 20 (Fig. 4). While the soluble sugars and starch concentrations in leaves and fruits of cv. Moneymaker were relatively stable at both temperatures, they were more affected in Susy3-IL at SOT. The contents of the reducing sugars glucose and fructose in cv. Moneymaker leaves were approximately 0.8 and $1.8 \mathrm{mg} / \mathrm{g}$ FW respectively, whereas glucose and fructose contents in Susy3-IL leaves increased fourand eightfold at SOT compared to the counterparts at CT (Fig. 4A). Sucrose content was clearly reduced at SOT in leaves of both tomato genotypes, but more severely in Susy3-IL to non-detectable amounts (Fig. 4A). Overall, the total soluble sugar (TSS) content of cv. Moneymaker leaves was hardly affected by temperatures, whereas it was 2.5 -fold higher in Susy3-IL leaves at SOT compared to the counterpart at CT (Fig. 4B). On the other hand, leaf starch content of both genotypes appeared not be affected by temperatures, which was measured around $0.2 \mathrm{mg} / \mathrm{g} \mathrm{FW}$ (Fig. 4C). The leaf sucrose:starch ratio decreased sharply at low temperature in both genotypes, and that ratio appeared to be higher in cv. Moneymaker than in Susy3-IL (Table 1). As for fruits, TSS content in cv. Moneymaker young fruits was approximately $15 \mathrm{mg} / \mathrm{g} \mathrm{FW}$ at both temperatures, whereas it was almost $30 \mathrm{mg} / \mathrm{g} \mathrm{FW}$ in Susy3-IL's counterparts at CT but was reduced twofold at SOT (Fig. 4B). The starch content in cv. Moneymaker young fruits was around $0.1 \mathrm{mg} / \mathrm{g} \mathrm{FW}$ at both temperatures (Fig. 4C). Starch content was higher in Susy3-IL young fruits at CT with $0.25 \mathrm{mg} / \mathrm{g} \mathrm{FW}$, but it was 
reduced 2.5-fold at SOT. For the measurements of carbohydrate contents in ripe fruits, unfortunately the samples at $\mathrm{CT}$ were lost. For ripe fruits samples at SOT, the concentrations were 9.5 vs $10.4,8.2$ vs $4.9,13.8$ vs $13.2,31.5$ vs 28.5 , and 0.02 vs $0.20 \mathrm{mg} / \mathrm{g} \mathrm{FW}$ for sucrose, glucose, fructose, TSS, and starch content in cv. Moneymaker and Susy3-IL, respectively. The big differences were about half-fold decrease in glucose content and 10fold increase in starch content in ripe fruits of Suy3-IL compared to that of cv. Moneymaker.

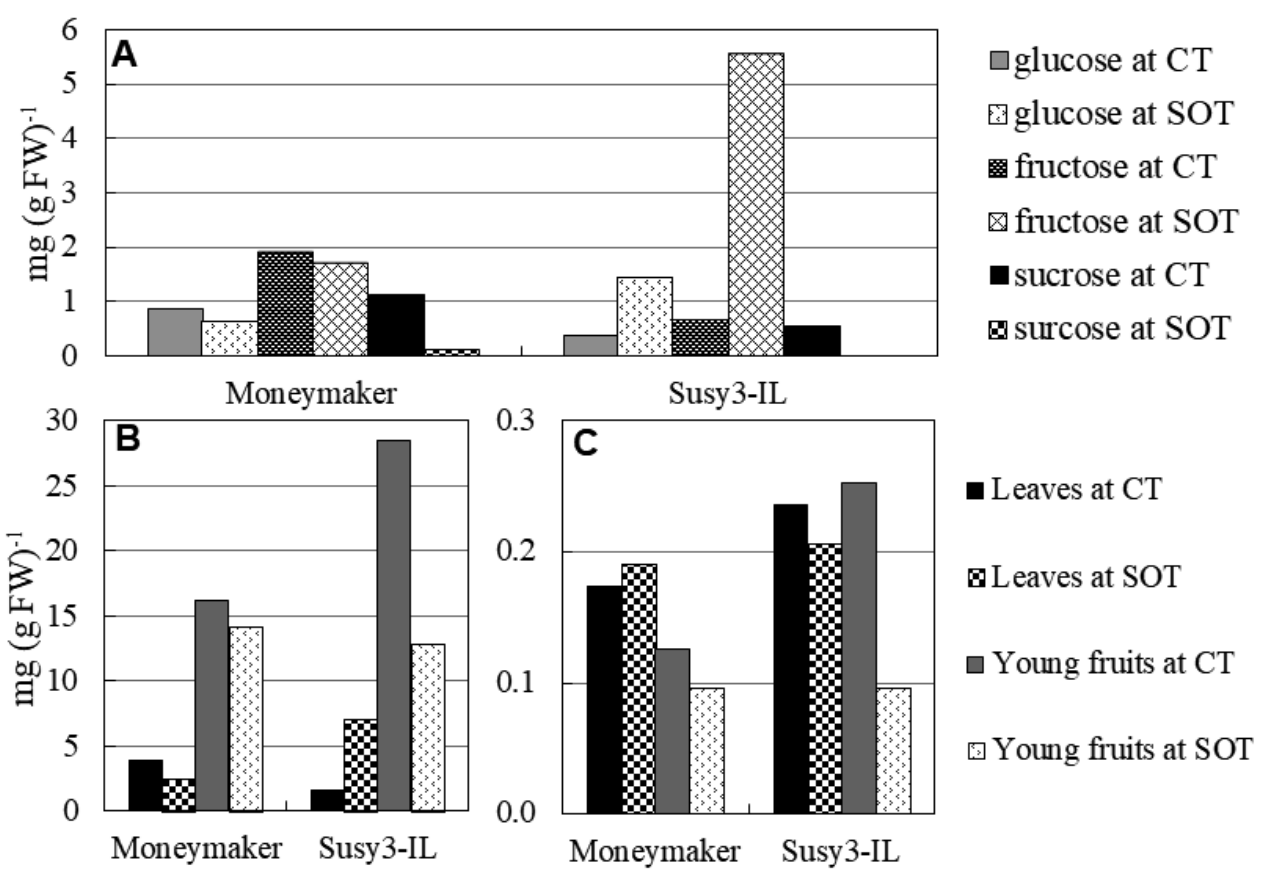

Figure 4. Soluble sugars and starch content in leaves and young fruits collected on week 20 from cv. Moneymaker and Susy3-IL. Panel A shows glucose (glu), fructose (fru), and sucrose (suc) contents in leaves separately. Panel B displays total soluble sugars in leaves and young fruits, calculated as the sum of glucose, fructose, and sucrose contents. Starch contents are shown in panel C. The units of all these measurement are $\mathrm{mg}\left(\mathrm{g} \mathrm{FW}^{-1}\right.$. The data in these panels are the mean value of technical duplicates derived from measurement on one single plant. 
Table 1: Leaf sucrose:starch ratios in week 20 (11)

\begin{tabular}{lcc}
\hline Genotype & $\mathrm{T}\left({ }^{\circ} \mathrm{C}\right)$ & Leaf sucrose:starch ratio \\
\hline Moneymaker & 25 & 6.5 \\
& 16 & 0.6 \\
Susy3-IL & 25 & 2.3 \\
& 16 & $\mathrm{n} / \mathrm{a} *$ \\
\hline
\end{tabular}

* The leaf sucrose:starch ratio at $16^{\circ} \mathrm{C}$ cannot be calculated because the sucrose content was too low to be detected.

\section{Different profiles in activity of enzymes associated with breakdown of sucrose}

Activities of sucrose synthase (SUSY) and invertase enzymes involved directly in the hydrolysis of sucrose were assayed at $25^{\circ} \mathrm{C}$ and $16^{\circ} \mathrm{C}$ in fruits harvested in week 20 at $\mathrm{CT}$ and SOT. SUSY activity in this study was total activity of all three SUSY1/3/4 isoforms, whereas the activity of cell-wall invertase (CWI) and vascular invertase (VIN) were measured separately. SUSY activity was highest in green fruits, but present in very low levels in ripe fruits at both growth temperatures (Fig. 5A and D). Furthermore, SUSY activity of Susy3-IL was consistently lower than that of cv. Moneymaker (see also Supplementary data 4 Table S2). For invertases, CWI was slightly more active in red fruits. CWI activity of Susy3-IL red fruits (Fig. 5B) was lower than that of cv. Moneymaker (Fig. 5E). Interestingly, CWI activity of ripe fruits of both genotypes harvested at SOT was higher than the counterpart at CT (see also Supplementary data 4 Table S1). On the other hand, VIN was very active in red fruits, up to sevenfold higher than in green fruits (Fig. 5C and F). Similar to CWI activity, VIN activity of Susy3-IL young and ripe fruits at SOT was also higher than the counterparts at CT (Fig. $5 \mathrm{~F}$ ). Furthermore both SUSY and VIN activities were lower at assay temperature $16^{\circ} \mathrm{C}$ compared to that at $25^{\circ} \mathrm{C}$, but $\mathrm{CWI}$ activity appeared not to be reduced at $16^{\circ} \mathrm{C}$ compared to that at $25^{\circ} \mathrm{C}$ in most samples. 


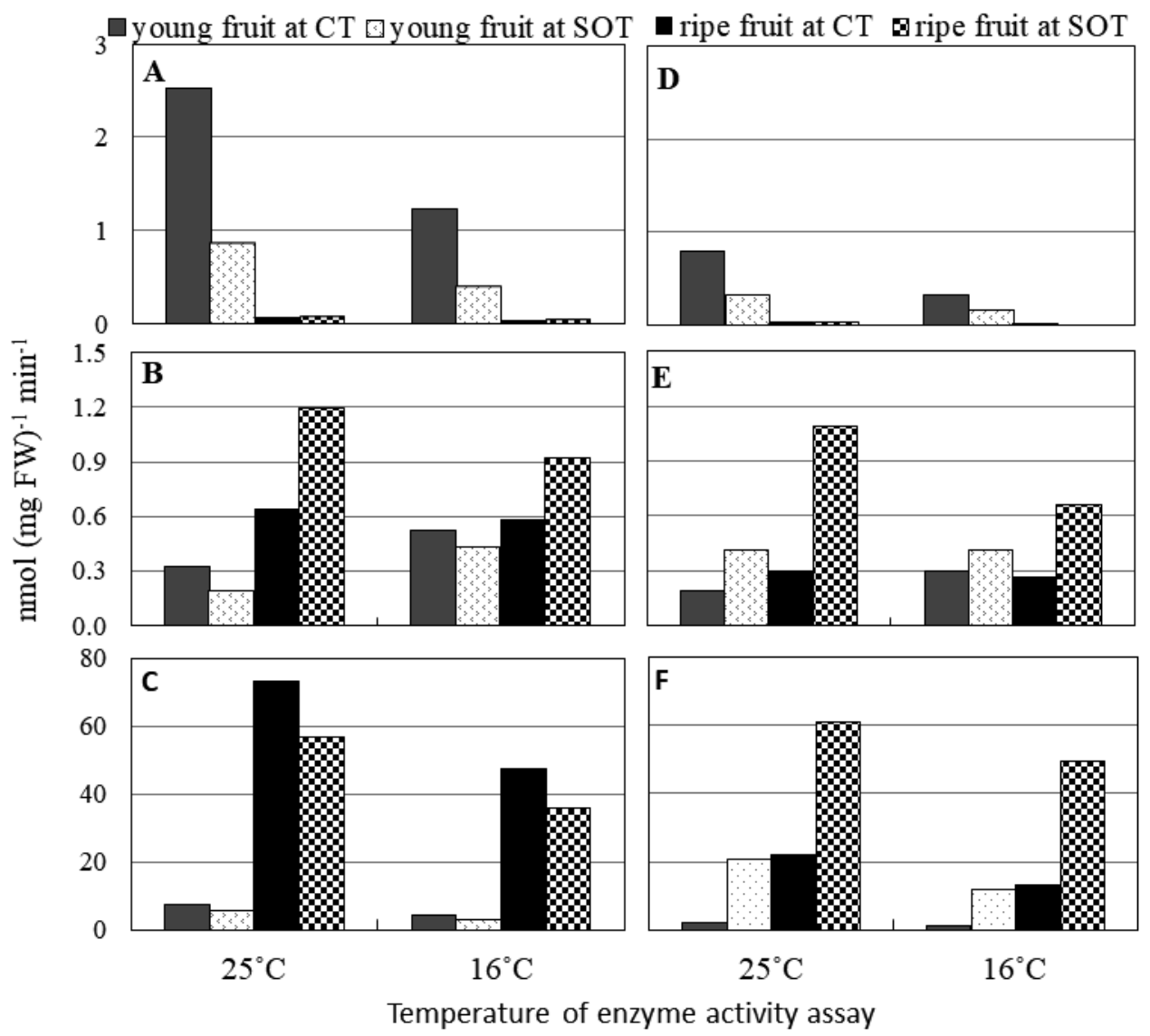

Figure 5. Activities of several sucrose hydrolyzing enzymes measured at $25^{\circ} \mathrm{C}$ and $16^{\circ} \mathrm{C}$ in young and ripe fruits of cv. Moneymaker (panel A - C) and Susy3-IL (panel D - F) harvested in week 20 at CT and SOT. Three enzymes were assayed for their activities: sucrose synthase (panel A and D), cell wall invertase (panel B and E), and vacuolar invertase (panel C and F). The data in these panels are the mean value of technical duplicates derived from measurement on one single plant.

\section{Genes encoding sucrose synthase and invertase are differentially expressed in distinct plant organs}

Expression of genes encoding the sucrolytic enzymes SUSY and INVs were studied in different plant organs in week 20. Those genes are Susy1/3/4 encoding sucrose synthases (SUSY), Lin5/6/7/8 for cell wall invertases (CWI), and Tiv1 for vacuolar invertase (VIN). As shown in Fig. 6, the expression of those genes was more variable among organ types, while the effect of temperature on gene expression was not clear (see also Supplementary data 5 Table S1). Among the Susy genes, the expression of Susy1 and Susy3 was highest in fruits and roots and lowest in leaves. The transcript level of Susy1 was abundant in both young and ripe fruits, meanwhile expression of Susy3 was higher in red fruits and roots. Susy4 
expression was highest in ripe fruits, but very low compared to Susy1 and Susy3 expression, i.e. 180 - 700-fold lower in roots and 3 - 60-fold lower in fruits. For invertase genes, expression of Tiv1 encoding VIN was highest in fruits, especially ripe fruits (Fig. 6D). Among the genes encoding CWI, the transcript of Lin 5 was the most abundant in fruits. The highest expression of $\operatorname{Lin} 6$ was in roots and leaves. $\operatorname{Lin} 8$ expression was also found relatively high in leaves but very low in other tissues, whereas $\operatorname{Lin} 7$ was the lowest expressed in all tissues. One striking observation was that in the ripe fruit tissues at CT, the expression of most of the studied genes, except Tiv1, was higher in Susy3-IL than in cv. Moneymaker (Fig. $6 \mathrm{D}$, Supplementary data 5 Table S2).

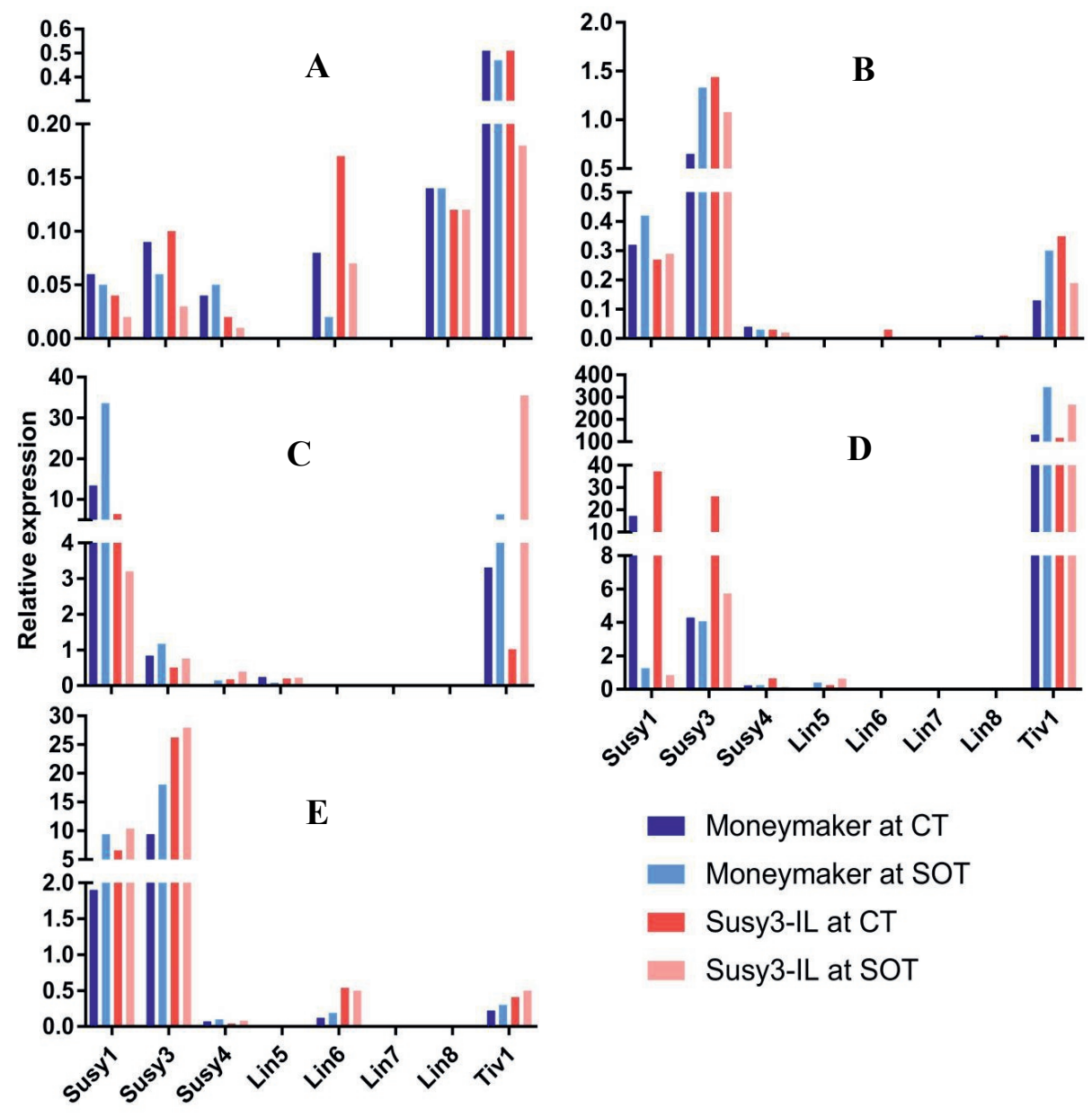

Figure 6. The expression of genes Susy1/3/4 encoding SUSYs, Lin5/6/7/8 encoding CWI, and Tiv1 encoding VIN in different tissues of cv. Moneymaker and Susy3-IL. The studied tissues are leaf (A), stem (B), young fruit (C), ripe fruit (D), and root (E). Relative expression of these was normalized against the expression of gene Act encoding actin. The data in these panels are the mean value of technical duplicates derived from measurement on one single plant. 


\section{Discussion}

\section{Both genotypes do not experience temperature stress on photosystem II}

The maximum, dark-adapted quantum efficiency of photosystem II photochemistry $\left(\mathrm{F}_{\mathrm{V}} / \mathrm{F}_{\mathrm{M}}\right)$ is commonly used to measure the accumulation of stress in plants, which is estimated as the ratio of variable chlorophyll fluorescence yield and the maximum fluorescence yield. The $\mathrm{F}_{\mathrm{V}} / \mathrm{F}_{\mathrm{M}}$ of $\mathrm{C} 3$ plants under optimal growth condition is around 0.83 and a value below 0.80 is generally taken to indicate the advent of photo-inhibition (Björkman and Demmig 1987). Since the $\mathrm{F}_{\mathrm{V}} / \mathrm{F}_{\mathrm{M}}$ of both Susy3-IL and recurrent cv. Moneymaker in this study were around $0.80-0.81$ in both CT and SOT (Fig. 1), this indicated that both genotypes did not experience temperature stress to the extent necessary to cause any damage to photosystem II.

\section{Introgression of Susy3 allele from wild species improves dry matter allocation to fruits at control temperature}

The introgression of a sucrose hydrolyzing allele, such as sucrose synthase, from a wild species in a commercial tomato background is of interest because it could play a role in improving the sucrose metabolism and plant sink strength. The preliminary results in this study show that wild allele Susy can have a positive influence on plant sink strength of a cultivated tomato by introgression. At 20 weeks after sowing, the introgression line Susy3-IL had $58 \%$ of DM allocated to fruits compared to $49 \%$ in the cv. Moneymaker background, a $9 \%$ increase DM fraction to fruits (Fig. 3D). This is a considerable improvement because the $\mathrm{DM}$ fraction to fruits is an important factor determining final fruit yield as well as fruit quality (Heuvelink and Dorais 2005). This higher DM allocation to fruits in Susy3-IL appeared to be gained at the expense of lower DM distribution to its leaves and stems compared to those in cv. Moneymaker (Fig. 3A). This observation was supported by the measured total soluble sugars and starch contents (Fig. 5), in which Susy3-IL green fruits had twofold higher total soluble sugar and starch concentrations compared to cv. Moneymaker fruits, and Susy3-IL leaves accumulated twofold less total soluble sugars compared to cv. Moneymaker leaves at CT. Despite of this encouraging 9\% increase in DM fraction to fruits, the overall performance of Susy3-IL was not as good as that of the recurrent parent cv. Moneymaker. This inferior performance of Susy3-IL in comparison to cv. Moneymaker was evident from the lower values of several important parameters such as total plant dry weight, fruit dry weight and number of fruits (Fig. 2). A possible explanation for these results may be the current generation (BC3S2) of Susy3-IL. At the BC3S2 generation, Susy3-IL can theoretically contain around $6 \%$ of the donor parent wild species S. neorickii G1.1601 genome, including homozygous segments of chromosome 7 and 12 (Supplementary data 1 Fig. S1). These 6\% genomic regions of wild species within Susy3-IL could contain hundreds to thousands of genes encoding many undesired traits from the wild species, also known as linkage drag. As a consequence, this linkage drag is likely the cause for the observed reduction in plant growth and development in Susy3-IL. Several genes located on tomato chromosome 7 and 12 have been reported to be involved in tomato fruit development and ripening as signaling factors such as proteobacterial-like RNA polymerases (Rpotp, Solyc07g005930), Golden 2-like 1 (Glk1, Solyc07g05363) genome uncoupled (Gun2, 
Solyc12g009470), a dual-function thylakoid protein kinase (Stn7, Solyc12g021280), etc. (reviewed in Pesaresi et al. 2014). The presence of such genes from the donor wild species parent in the cultivated tomato background might negatively influence the growth and vigor of the introgression line. Therefore, generating more advanced backcrossed lines to narrow down the introgressed region in the future could help to evaluate the effect of the introgressed allele better. An example of this approach is visible in the generation of the advanced BC population by crossing a processing tomato variety M82 with a green fruited wild species $S$. pennellii to identify and map yield-related QTL (Eshed and Zamir 1995). Further studies with this population resulted in the identification of a specific line that carried the QTL Brix-9-2-5 mapped within the flower and fruit specific invertase (Lin5) in chromosome 9 and had an increase in sugar yield compared to the elite variety (Fridman et al. 2002, Fridman et al. 2004).

\section{Susy3 introgression line does not perform well at sub-optimal temperature}

Having a new tomato variety that could perform well at SOT is one of the approaches to reduce energy consumption for the locations where tomatoes need to be grown in heated glasshouses. Both cv. Moneymaker and Susy3-IL were shown to have their growth and development profoundly reduced at SOT. The performance of Susy3-IL at SOT was even poorer than that of the recurrent parent cv. Moneymaker. At the morphological level, plants grown at SOT showed sharply reduced specific leaf area and their leaves got thicker (Fig. 2CD), as has been shown in other studies as well (Venema et al. 1999b, Atkin et al. 2006a, Kläring et al. 2015). Furthermore, the reduction in leaf sucrose content (Fig. 4A) and sucrose:starch ratio at SOT (Table 1) was also matched with the poor plant growth and development (Fig. 4A). The leaf sucrose content in Susy3-IL was consistently lower than the counterpart of cv. Moneymaker, and was not detectable at SOT. This non-detectable amount of sucrose in Susy3-IL leaves in week 20 was accompanied with lowest values in total leaf area, number of fruits, total plant dry weight, and fruit dry weight (Fig. 2). It has been shown in several species, such as winter rye, wheat, Arabidopsis, and tomato, that high leaf sucrose:starch ratio is an important aspect for plants to acclimate to low temperatures (Hurry et al. 1995, Savitch et al. 1997, Strand et al. 1997, Strand et al. 1999, Dinh et al. 2019). Therefore, more attention should also be paid to other players involved in the sucrose synthesis in leaves such as sucrose phosphate synthase (SPS) and sucrose phosphate phosphatase (SPP) when generating more advanced introgression lines. These two enzymes SPS and SPP have been reported to form a complex in planta to facilitate the synthesis of sucrose from sucrose-6-phosphate (Maloney et al. 2015). In addition, those authors also observed enhanced plant growth in the transgenic Arabidopsis and hybrid poplar plants containing the fusion construct between SPS-SPP. 
Activity of enzymes involved in degradation of sucrose does not explain the total soluble sugar profile in fruits

Activities of sucrose hydrolyzing enzymes were measured on fruits as the tomato major sink organs because these enzymes have direct impact on sucrose metabolism and subsequently on plant sink strength. SUSY activity was very high in green fruits but almost absent in red fruits in both Susy3-IL and cv. Moneymaker (Fig. 5A and D). This was in agreement with several other studies (Robinson et al. 1988, Yelle et al. 1988, Wang et al. 1993a, Wang et al. 1994, Islam et al. 1996). Also in line with the finding in Islam et al. (1996), VIN activity was highest in ripe fruits but very low in young fruits, while CWI activity was less active in fruits compared to VIN (Fig. 5B, C, E, F). However there was no relation between the activities of these enzymes and the total soluble sugar concentration in young fruits of Susy3-IL and cV. Moneymaker (Fig. 5A). Furthermore, SUSY activity in Susy3-IL fruits was even lower than that in cv. Moneymaker. Since there is no method available to measure activity of different SUSY isoforms separately from fruit extracts (i.e. SUSY1, SUSY3, and SUSY4), we could not infer the activity of SUSY3 in Susy3-IL from the wild species S. neorickii G1.1601 compared to that from cv. Moneymaker. Therefore the effect of the introgression of wild Susy3 allele on the overall SUSY activity in fruit of Susy3-IL is still unknown. However, the observation of high and low activity of SUSY in young and ripe fruits respectively in the present study agrees with previous report (Steinhauser et al. 2010). Interestingly while the SUSY activity was consistently decreased in fruits harvested at SOT, activity of VIN and CWI of Susy3-IL fruits grown at SOT was higher than the counterparts at CT. These observations need to be further investigated.

\section{Expression of genes associated with breakdown of sucrose are more influenced by tissue type than by temperature}

Expression of sucrose synthase and invertase encoding genes was not consistently influenced by temperature but very tissue-specific (Fig. 5 and Supplementary data 4 Table S1). Susy 1 and Susy3 were highly expressed in sink tissues such as fruits and roots but lowest in the source tissue leaves, whereas Susy4 had similar expression pattern but was lower in magnitude. These findings were in agreement with Goren et al. (2011). Focusing on fruits, Susy1 and Susy3 transcript level was highest in young fruits and ripe fruits respectively, which was consistent with other reports (Wang et al. 1994, Chengappa et al. 1999, Kortstee et al. 2007). Lin5 was highly expressed in fruit tissues, which was also observed in other studies (Fridman and Zamir 2003, Kortstee et al. 2007). In addition, Lin5 transcript accumulation was higher in fruits at SOT. The $\operatorname{Lin} 7$ gene resides on chromosome 9 and is arranged in a direct tandem with Lin 5 , about $1.5 \mathrm{~kb}$ apart from each other (Fridman and Zamir 2003). However $\operatorname{Lin} 7$ was hardly expressed in leaves, stems, roots, or fruits. This could be explained by previous findings that $\operatorname{Lin} 7$ expression was specific to flowers that were not included in this study (Godt and Roitsch 1997, Fridman and Zamir 2003). Meanwhile, the expression of Lin6 and Lin8 located on chromosome 10 was highest in leaves as reported earlier (Fridman and Zamir 2003). The detection of the higher transcript level of Tivl in ripe fruits compared to young ones in cv. Moneymaker and Susy3-IL are supported by previous 
findings (Klann et al. 1993, Miron et al. 2002, Kortstee et al. 2007). In addition, a relation between the expression of Tiv1 and Lin5 and activity of the encoding enzyme VIN and CWI respectively was observed in fruits at both temperatures (Fig. 5 and Fig. 6). These results confirm that VIN and CWI are regulated at transcriptional level, which supports earlier report (Kortstee et al. 2007). On the other hand, it was interesting to observe negative relation in expression level of Susy3 and SUSY activity in fruit of both genotypes. This observation is in line with the finding of Steinhauser et al. (2010), in which the transcript level of SGNU143650 unigene corresponding to Susy3 was shown to have a negative correlation with activity of SUSY in tomato fruit pericarp during fruit development. However, the gene expressions reported by those authors were from TOM1 cDNA array which did not have the expression data of other Susy alleles.

\section{Conclusion}

Introgression of a wild allele into cultivated tomatoes is one of the few options to enhance or create new traits. At the current state, Susy3-IL that carries an introgressed chromosome fragment containing the Susy3 allele from wild accession S. neorickii G1.1601 in cv. Moneymaker background does not show an improved growth and development relatively to the cv. Moneymaker background at both CT and SOT. Yet, the preliminary result indicates an improvement in DM allocation to fruits of Susy3-IL compared to the parental cv. Moneymaker background at CT. This increase in DM allocation in Susy3-IL appears to have a relation with the higher soluble sugar and starch contents in its fruits. Interestingly neither expression nor activities of sucrolytic genes or enzymes explains that increase in DM allocation in Susy3-IL. Further investigation with more advanced introgression lines with smaller introgressed segments from the wild relative parent might help to elucidate the factor(s) that contribute to the increase in DM allocation in sink fruits, as well as broaden our understanding of the potential of wild alleles in the improvement of tomato performance at standard growing conditions as well as at suboptimal conditions.

\section{Acknowledgements}

This work was carried out within the research programme of BioSolar Cells co-financed by the Dutch Ministry of Economic Affairs, STW project number 5769, and Plant Breeding Wageningen University \& Research. The authors thank Yuling Bai and Richard Finkers for helping to identify ILs; Andre Maassen from Unifarm WUR for taking good care of the plants; Yi Li, Octavio Salazar Moya, Tulipan Zollinger, Nicolas Kaspric, and Emeline Pollet at Plant Breeding for helping with plant phenotyping; Yuniati Dinh for helping to make Figure 6. 


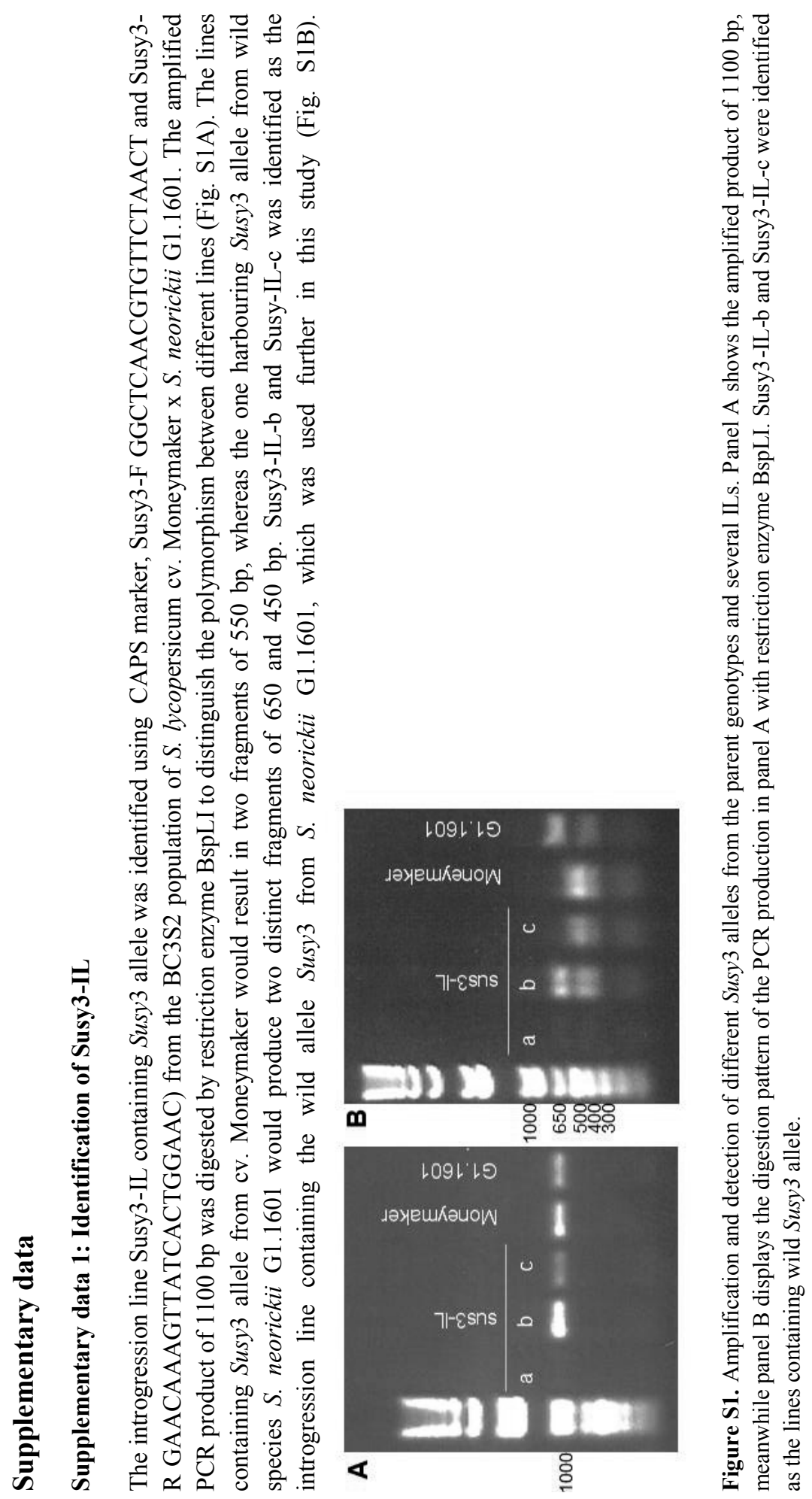




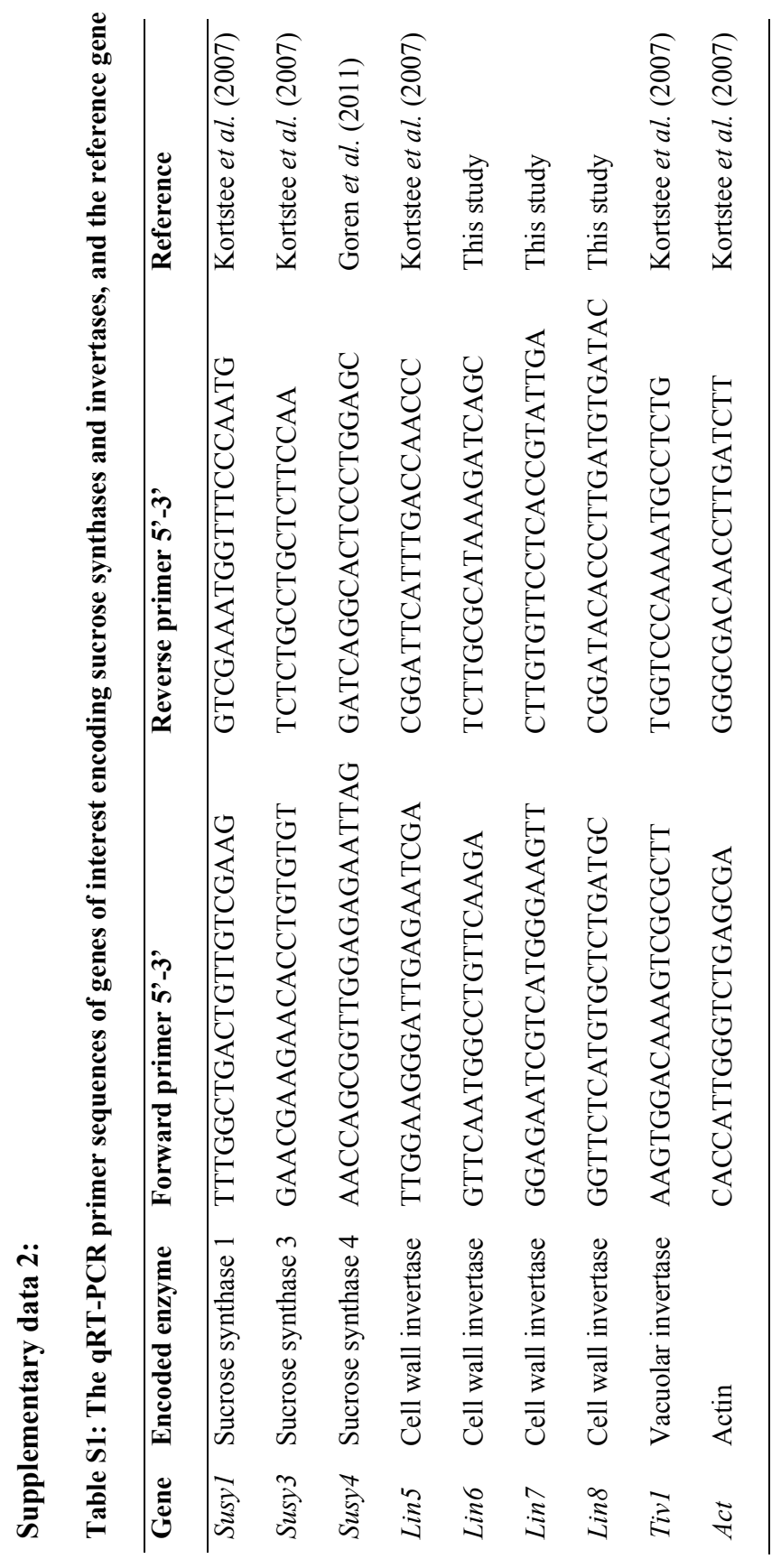


Supplementary data 3:

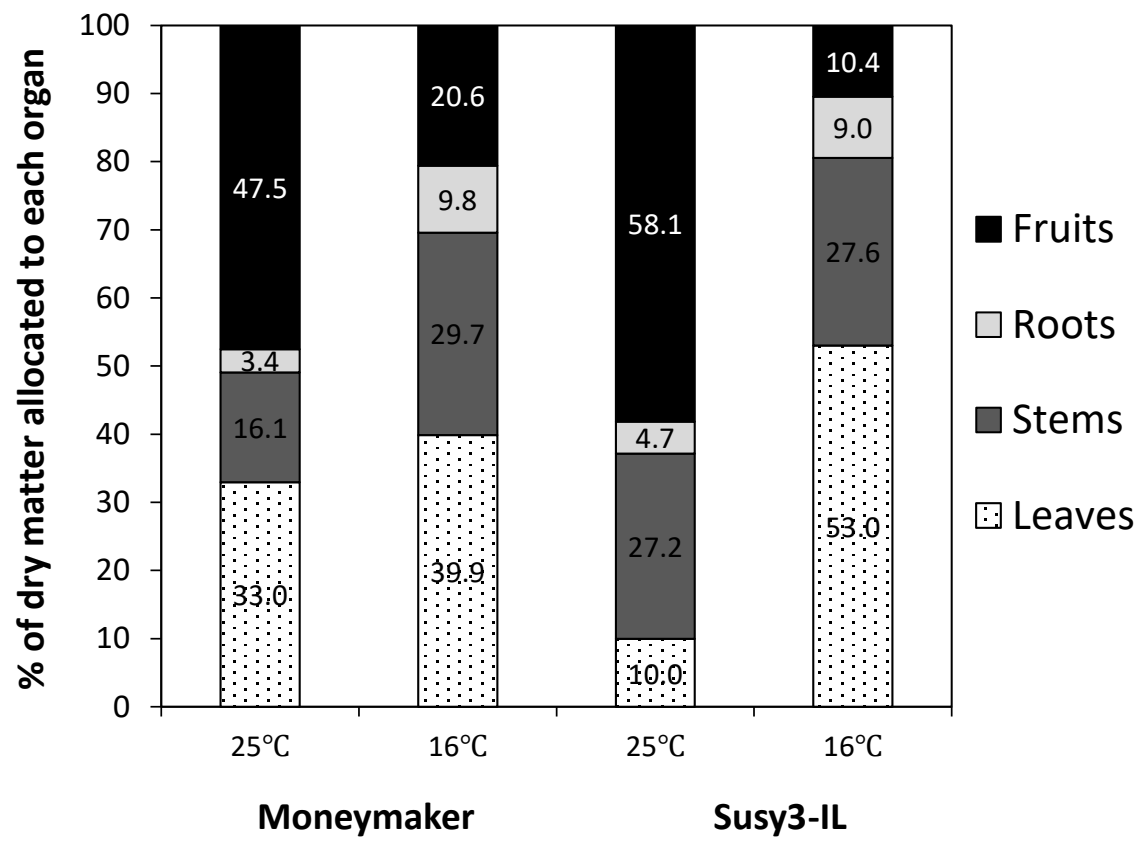

Figure S1: Fraction of DM allocation to different plant organs in week 20 at CT $(25 \circ \mathrm{C})$ and after 11 weeks exposed to SOT $(16 \circ \mathrm{C})$. 


\section{Supplementary data 4: Ratio of enzyme activities corresponding to temperatures and}

\section{genotypes}

Table S1. Ratio of enzyme activities in young and ripe fruits of cv. Moneymaker and Susy3-IL between sub-optimal (SOT) and control (CT) growth temperatures

\begin{tabular}{lllllll}
\hline Ratio SOT:CT & \multicolumn{2}{l}{ SUSY } & \multicolumn{3}{c}{$\mathrm{CWI}$} & \multicolumn{2}{c}{ VIN } \\
\cline { 2 - 7 } Assay temperature & $25^{\circ} \mathrm{C}$ & $16^{\circ} \mathrm{C}$ & $25^{\circ} \mathrm{C}$ & $16^{\circ} \mathrm{C}$ & $25^{\circ} \mathrm{C}$ & $16^{\circ} \mathrm{C}$ \\
\hline Moneymaker-Young fruit & 0.3 & 0.3 & 0.6 & 0.8 & 0.8 & 0.6 \\
Moneymaker-Ripe fruit & 1.2 & 2.0 & 1.9 & 1.6 & 0.8 & 0.8 \\
Susy3-IL-Young fruit & 0.4 & 0.5 & 2.2 & 1.4 & 10.2 & 10.3 \\
Susy3-IL-Ripe fruit & 1.0 & 2.0 & 3.8 & 2.4 & 2.8 & 3.7 \\
\hline
\end{tabular}

Values highlighted in light and dark grey are ratio with minimum two-fold decrease and increase, respectively.

Table S2. Ratio of enzyme activities between cv. Moneymaker and Susy3-IL fruits

\begin{tabular}{lllllll}
\hline Ratio SUSY3-IL:Moneymaker & \multicolumn{2}{l}{ SUSY } & \multicolumn{3}{c}{$\mathrm{CWI}$} & \multicolumn{3}{l}{ VIN } \\
\cline { 2 - 7 } Assay temperature & $25^{\circ} \mathrm{C}$ & $16^{\circ} \mathrm{C}$ & $25^{\circ} \mathrm{C}$ & $16^{\circ} \mathrm{C}$ & $25^{\circ} \mathrm{C}$ & $16^{\circ} \mathrm{C}$ \\
\hline Young fruit at CT & 0.3 & 0.3 & 0.6 & 0.6 & 0.3 & 0.3 \\
Young fruit at SOT & 0.4 & 0.4 & 2.2 & 0.9 & 3.6 & 4.1 \\
Ripe fruit at CT & 0.5 & 0.5 & 0.5 & 0.5 & 0.3 & 0.3 \\
Ripe fruit at SOT & 0.4 & 0.5 & 0.9 & 0.7 & 1.1 & 1.4 \\
\hline
\end{tabular}

Values highlighted in light and dark grey are ratio with minimum two-fold decrease and increase, respectively. 


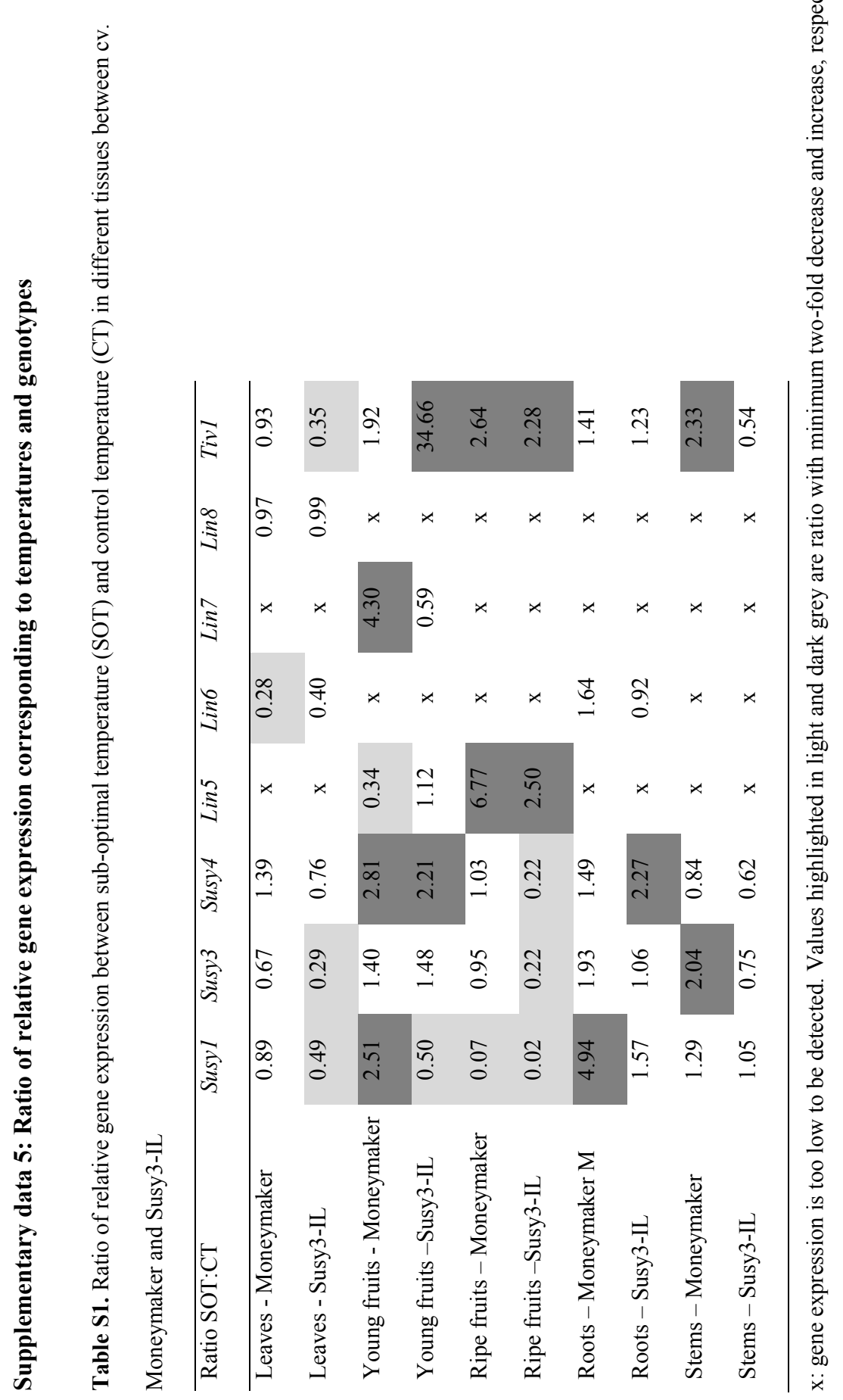




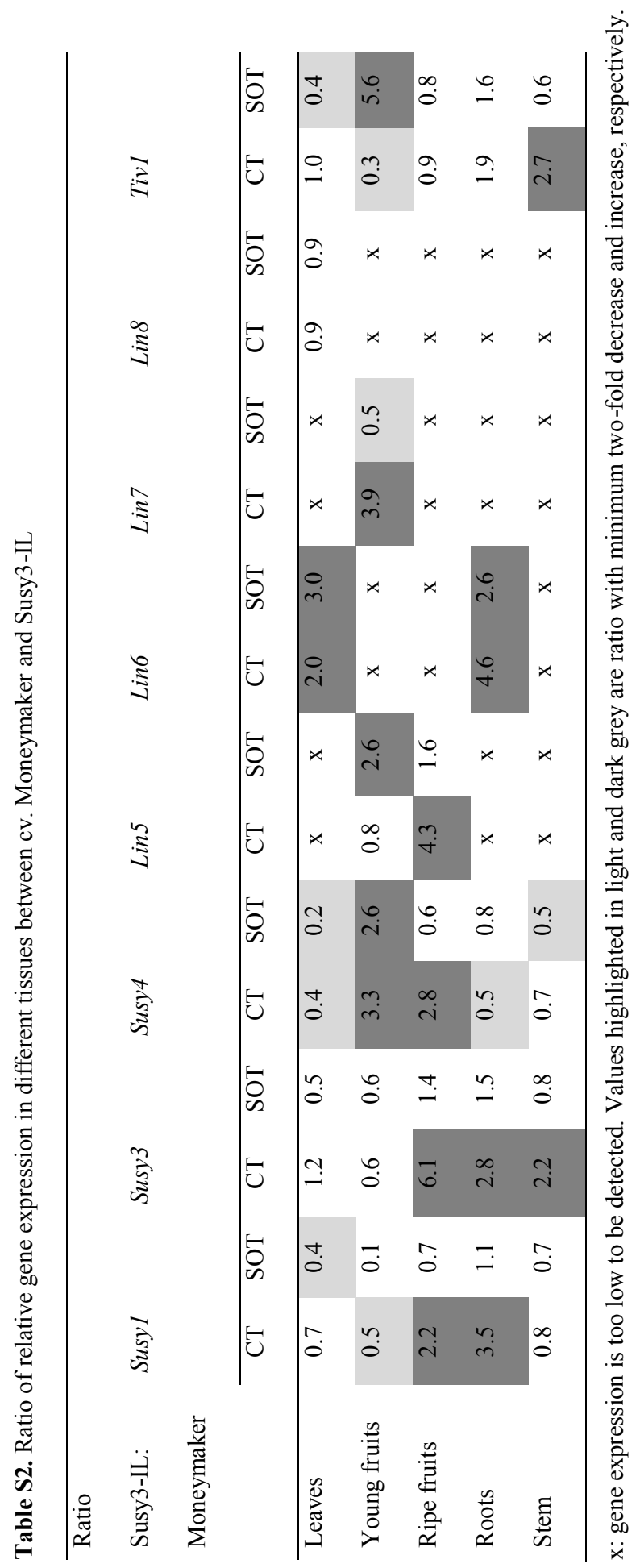





\section{Chapter 5}

\section{General Discussion}

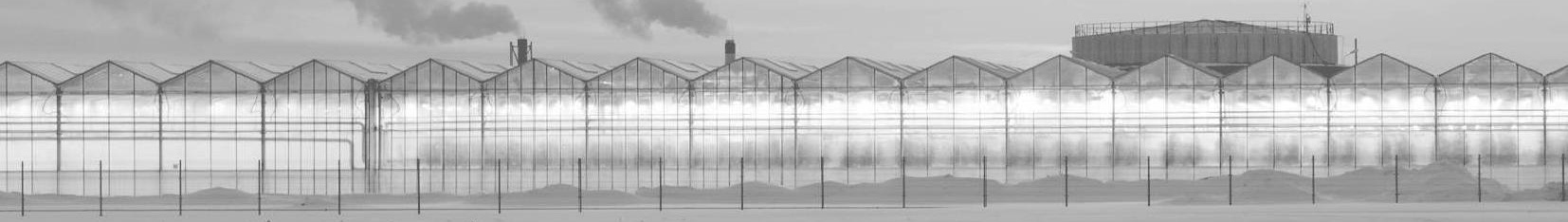

*a d

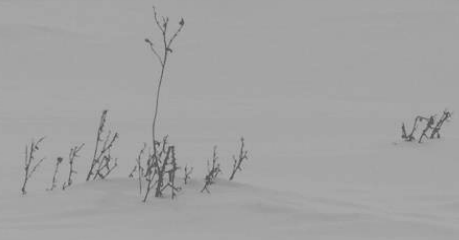


In northern areas with temperate climate such as the Netherlands, crops originating from (sub) tropical areas such as tomatoes are usually grown in heated greenhouses. The current economical optimum growth temperature for tomato cultivation in heated greenhouses in the Netherlands is approximate $19-20^{\circ} \mathrm{C}$ (van der Ploeg and Heuvelink 2005). With continuous innovations in greenhouse technologies (e.g. new building materials, more controls over growth conditions such as temperature, $\mathrm{CO}_{2}$ enriching and lighting supply), the greenhouse growers have significantly improved the energy efficiency compared to the past decades, mainly based on higher yield per unit cultivated area (van der Knijff et al. 2004, van der Velden and Smit 2018). The upholding of optimal temperatures in heated greenhouses in cold climate areas is, however, costly and environmentally unfriendly. The development of new varieties that are able to perform well at lower growth temperature without compromising on the yield and quality would overcome these economic and environmental bottlenecks. This is especially important for the Netherlands which aims for a fully sustainable and economically viable energy supply by 2050 (WUR and Kas als Energiebron 2016).

This thesis addresses different aspects underlying sub-optimal temperature (SOT) tolerance in tomato. SOT is defined as the temperature below the current economical optimum growth temperature in heated greenhouse in the Netherlands of $19-20^{\circ} \mathrm{C}$ and above the chilling temperature of $12^{\circ} \mathrm{C}$. For that, I studied: the effects of SOT on plant growth and development, photosynthetic capacities, and metabolic profiles in source leaves and sink fruits between the high altitude wild relative Solanum arcanum LA385 (LA385, originally grown at 2,400 m above sea level) and Solanum lycopersicum cultivar Moneymaker (cv Moneymaker) from the flower onset till later stages of fruit development (Chapter 2). In Chapter 3 the natural variation present in a key enzyme involved in the hydrolysis of sucrose - sucrose synthase (SUSY), was mapped in 85 tomato accessions and the effect of several different variants were correlated with SUSY kinetics at SOT. Furthermore, the effects of SOT on plant growth and development, dry matter allocation, gene expression, enzyme activity, and metabolite profiles were evaluated on an introgression line carrying a Susy3 allele from the high altitude wild relative Solanum neorickii G1.1601 (also known as LA0735) and compared with the cv Moneymaker background (Chapter 4). In the following sections, I will discuss the major findings from these experiments and make recommendations for future research concerning breeding for SOT tolerance.

\section{Sink strength and leaf morphology play important roles in the acclimation to SOT in S. arcanum LA385}

Breeding efforts in tomato have mostly focused on yield related (including resistance to biotic and tolerance to abiotic stresses) and quality (such as taste and color) traits. Little has been done on the breeding for tomato cultivars that grow efficiently under SOT. As this trait in presumably not present in commercial varieties, the breeding for cultivars that perform efficiently below optimal temperatures requires the use of wild species tomato that can perform better than the cultivated ones at SOT. To our knowledge, the majority of the reports evaluating tolerance to lower temperatures in different tomato species has only been done at the vegetative phase, while thermophilic plants like tomatoes are most sensitive to 
temperature stress at the reproductive phase (Zinn et al. 2010). In Chapter 2, we attempted to bridge this gap by studying various aspects of photosynthesis, biomass allocation, and sugar metabolism in the high altitude wild species $S$. arcanum LA385 at SOT $\left(16 / 14^{\circ} \mathrm{C}\right.$ day/night) and control temperature $\left(\mathrm{CT}, 22 / 20^{\circ} \mathrm{C}\right.$ day/night) from flower onset till a later stage of fruit development, and compared them to those in cv Moneymaker. As expected, LA385 appeared to acclimate to SOT much better than cv Moneymaker, which was most clear in the plants 16 weeks after sowing (11 weeks at SOT). For instance, we observed similar values in LA385 plants at both CT and SOT for specific leaf area (SLA), total plant fresh weight and dry weight, dry matter allocation to fruits, accompanied with significant increase in parameters such as total leaf area (TLA) and fruit numbers at SOT than CT, whereas those parameters in cv Moneymaker were still profoundly affected by SOT (Table 1 and 2 in Chapter 2). Could this be the result of LA385's higher photosynthetic capacity linked to source strength [e.g. such as photosystem II operating efficiency $\left(\Phi_{\mathrm{PSII}}\right)$, net $\mathrm{CO}_{2}$ assimilation rate $\left(\mathrm{A}_{\mathrm{N}}\right)$ and lightsaturated $\mathrm{CO}_{2}$ assimilation rate $\left(\mathrm{A}_{\text {sat }}\right)$ ] or to higher sink strength with higher number of fruits in this case? The photosynthetic parameters reported in Table 3 in Chapter 2 suggest that that those parameters in LA385 were not responsible for the superior acclimation to SOT, because they were all slightly lower at SOT than at CT, even though they were consistently higher in LA385 than in cv Moneymaker at SOT. One observation, not discussed in Chapter 2, was an alteration in leaf morphology under SOT. While LA385's leaves were horizontal in both growth temperatures, cv Moneymaker's leaves were horizontal at CT but curly at SOT. The same curling leaf morphology at SOT was also observed in Susy3-IL - an introgression line containing the Susy3 allele from the wild species S. neorickii G1.1601, which performs badly at SOT relative to CT (Chapter 4). Nederhoff et al. (1992) reported that leaf curling occurred in tomato plants with low sink-source ratio. In addition, low sink-source ratio leading to reduction in specific leaf area (SLA) was also reported in cucumber (Marcelis 1991) and tomato (Heuvelink and Buiskool 1995). Higher sink strength with higher number of fruits and leaf morphology seem to play important roles in LA385's superior acclimation ability to SOT compared to that in cv Moneymaker.

\section{No relation between accumulation of phosphorylated intermediates and photosynthesis inhibition}

Several studies have reported a reduction in the rate of photosynthesis and of sucrose synthesis caused by the accumulation of phosphorylated intermediates in leaves of plants exposed to abrupt lowering of temperature (Labate and Leegood 1988, Strand et al. 1997). This phenomenon is also known as feedback inhibition of photosynthesis. In Chapter 2, we did not observe a clear increase in phosphorylated mono and di-saccharides at SOT. Out of the five measurable phosphorylated sugars (i.e. suc-6-P, glu-1-P, glu-6-P, man-6-P, and fru6-P) in leaf samples, only the accumulation of Glu-6-P was higher at SOT than at CT in both LA385 and cv Moneymaker in week 16 (Fig. 2 in Chapter 2). The fact that accumulation of phosphorylated sugars in our study remained constant could be related to the developmental stage of the leaf samples that were used for the measurements. In our study, we used the youngest fully mature leaf, which were likely the developed leaves at SOT, especially in week 16. These SOT-developed leaves might have had enough time to adapt their metabolic 
profiles, thus did not show the accumulation in most of the measured phosphorylated sugars. Interestingly, Hurry et al. (1995) reported that in plants grown at $5^{\circ} \mathrm{C}$ the total hexosephosphate contents increase by $120 \%$ and $30 \%$ in cold-hardened and non-cold-hardened winter rye leaves, respectively. The authors also showed that this increase of hexosephosphate pool positively correlated with the recovery of photosynthesis in cold-hardened leaves. In the same study, those authors suggested that a large increase in hexose-phosphate pool in leaves of cold-hardened winter rye compensates for the higher substrate threshold concentrations needed for activation of crucial enzymes related to photosynthetic carbonreduction cycle and of sucrose synthesis, such as ribulose-1,5-biphoshate carboxylase/oxygenase (Rubisco) and sucrose-phosphate synthase (SPS). However we did not observe such high increase in hexose-phosphate pool in wild accession LA385 in our study. The large differences in temperature ranges $\left(16^{\circ} \mathrm{C} \mathrm{vs} 5^{\circ} \mathrm{C}\right)$ and species (tomato vs rye) could (partly) explain the different responses. As indicated above, glu-6-P showed a higher accumulation in leaves in both genotypes at SOT compared to CT, this appeared to parallel with the reduction in photosynthetic parameters, such as $\Phi_{\mathrm{PSII}}, \mathrm{A}_{\mathrm{N}}$ and $\mathrm{A}_{\text {sat }}$, in cv Moneymaker but not (significantly) in LA385. In leaves, glu-6-P together with UDP-glu is converted to trehalose-6-phosphate (T6P) by T6P-synthase (TPS), and the subsequent formation of trehalose from T6P is catalyzed by T6P phosphatase (TPP) (Blázquez et al. 1998, Vogel et al. 1998, De Smet et al. 2000, Elbein et al. 2003). Previous reports showed that the overexpressing of a gene encoding TPS in Arabidopsis resulted in a higher T6P content and photosynthetic capacity, but lower leaf area compared to the control plants (Pellny et al. 2004). Lunn et al. (2006) reported that the T6P concentration in Arabidopsis leaves is extremely low, ranging from 10 to $1000 \mathrm{pmol} \mathrm{g}^{-1}$ fresh weight. This range of concentration is very low compared to $0.05 \mathrm{nmol} \mathrm{mg}^{-1}$ dried weight which is the lowest detectable concentration of man-6-P or fruc-6-P in our study (or approximately $94 \mathrm{mmol} \mathrm{g}$ fresh weight based on leaf dry matter content of 12\%) (Fig. 2 in Chapter 2). In addition, those authors also noted that great care needs to be taken for baseline separation of T6P from suc-6-P - its most common isomer. Therefore, further research is needed to shed light on the involvement of T6P on photosynthetic capacity of cv Moneymaker and wild accession LA385 at SOT.

\section{High sucrose:starch ratio plays a role in plant's ability to acclimate to SOT}

Carbohydrate metabolism is known to play an important role in plant's responses to abiotic stresses (Rosa et al. 2009, Pommerrenig et al. 2018). In Chapter 2 we have observed some distinct carbohydrate profiles in two different tomato genotypes when grown at CT and SOT. While starch content in leaves of both cv Moneymaker and LA385 was significantly higher at SOT than at CT, the leaf neutral sugar contents were very distinct in those two genotypes (Fig. 3 in Chapter 2). The leaves of cv Moneymaker appeared to accumulate more hexoses at SOT, while their leaf sucrose content was less affected. In contrast, LA385 leaves had lower hexose content and higher and more stable sucrose content at SOT. The high leaf sucrose:starch ratio appeared to play an important role in the plant's ability to acclimate to SOT. The sucrose:starch ratio in leaves was approximately 10-fold higher in LA385 than in cv Moneymaker, i.e. 30 and 70\% for LA385 whereas 4 and 7\% for cv Moneymaker in week 
16 at SOT and CT respectively. This finding is in agreement with several studies on other species, such as winter rye, wheat, and Arabidopsis in which it has been reported that they acclimate to low temperatures by increasing their leaf sucrose:starch ratio (Hurry et al. 1995, Savitch et al. 1997, Strand et al. 1997, Strand et al. 1999). In Chapter 4, the preliminary data showed that the introgression line containing the Susy3 allele from wild species S. neorickii G1.1601 (Susy3-IL) had non-detectable levels of sucrose in its leaves, leading to a sharp reduction in sucrose:starch ratio at SOT compared to that at CT (Table 3). At the same time, Susy3-IL also suffered a drastic decrease in total biomass production at SOT relative to that at CT (Fig. 2). Is it a universal mechanism for plants to direct more assimilates to sucrose synthesis over starch formation in order to acclimate to low growing temperatures? In plants, sucrose phosphate synthase (SPS) catalyzes the synthesis of sucrose-6-phosphate (S6P) using UDP-glucose and fructose-6-phosphate as substrates. Subsequently, S6P is converted to sucrose and inorganic phosphate molecule (Pi) by sucrose phosphate phosphatase (SPP). Sun et al. (2011) have demonstrated that mutant Arabidopsis with reduced SPS activity (especially SPSA1) in leaves has a decrease in sucrose synthesis, an increase in starch synthesis and starch turnover in leaves (i.e. low sucrose:starch ratio) at low temperature. Those authors also observed that there were no differences in light saturated $\mathrm{CO}_{2}$ assimilation rate $\left(\mathrm{A}_{\text {sat }}\right)$, electron transport rate (ETR) between the null spsal mutants and wild-type at 400 ppm $\left[\mathrm{CO}_{2}\right]$. At $800 \mathrm{ppm}\left[\mathrm{CO}_{2}\right]$, there were $20 \%$ reduction in $\mathrm{A}_{\text {sat }}$, ETR, and leaf maximum ETR $\left(\mathrm{J}_{\max }\right)$, but no difference in leaf maximum Rubisco carboxylate rate $\left(\mathrm{V}_{\mathrm{cmax}}\right)$. They also concluded that the results indicate a limitation of triose-phosphate utilization (TPU) in the null spsal mutants at elevated $\left[\mathrm{CO}_{2}\right]$. However, those authors did not observe any difference in rosette growth between the null spsal mutants and wild-type at the normal growth condition with ambient $\left[\mathrm{CO}_{2}\right]$. They concluded that the higher accumulation of starch in leaves served as a transient sink, being remobilized during the night, which helps the null spsal mutants to maintain their growth. In tomatoes, overexpression of SPS has been reported to affect positively plant growth and yield in several studies. Worrell et al. (1991) started the investigation by overexpressing maize SPS cDNA under control of the Rubisco small subunit promoter in a cultivated tomato background ( $S$. lycopersicum cv UC82B). The authors observed in young plants a reduction in leaf starch content and an increase in sucrose content in transgenic lines having higher SPS activity in their leaves compared to the control. These transformed lines with elevated SPS activity were subsequently also studied in several other studies (Galtier et al. 1993, Micallef et al. 1995, Nguyen-Quoc et al. 1999). Galtier et al. (1993) focused on the single SPS-transformed tomato line (line 3812-9) with 8-fold elevation of SPS activity compared to the control at vegetative stage. The authors reported those transformed plants have higher leaf sucrose:starch ratio, higher shoot:root ratio, but no difference in net photosynthetic rate at ambient $\left[\mathrm{CO}_{2}\right]$ and low irradiances $\left(<400 \mathrm{~mol} \mathrm{~m} \mathrm{~s} \mathrm{~s}^{-2}\right.$ ${ }^{1}$ ), but $20 \%$ increase in the light- and $\mathrm{CO}_{2}$-saturated rate of photosynthesis compared to the control. However there is no difference in the total biomass production between all the lines at six weeks after sowing. Micallef et al. (1995) grew the transformed lines with high SPS activity at high light $\left(1000 \mathrm{~mol} \mathrm{~m}^{-2} \mathrm{~s}^{-1}\right)$ and at either ambient $(350 \mathrm{ppm})$ or high $(650 \mathrm{ppm})$ $\left[\mathrm{CO}_{2}\right]$ until the plants produced fruits. Those authors reported similar results to the findings at ambient $\left[\mathrm{CO}_{2}\right]$ as in Galtier et al. (1993). Micallef et al. (1995) also found an increase of $20 \%$ in leaf photosynthetic rate at $650 \mathrm{ppm}\left[\mathrm{CO}_{2}\right]$, but not at ambient $\left[\mathrm{CO}_{2}\right]$, in transformed plants 
relative to the control. Furthermore, they also reported a reduction in number of days to $50 \%$ blossom at both $\left[\mathrm{CO}_{2}\right]$ conditions in high-SPS-transformed lines relative to the control. Those authors also reported a significant increase of 1.5 times in fruit number, higher fresh and dry fruit weights, and earlier first harvest in transformed lines compared to the control at ambient [CO2], but interestingly not at high $\left[\mathrm{CO}_{2}\right]$. Nguyen-Quoc et al. (1999) used the same SPSoverexpressed tomato line 3812-9 (also known as SSU-9), but focused more on fruits as the major sink organs at 20-days-after-anthesis - the tomato fruit development stage where their sink activity is reported to be at the highest level (Ho et al. 1987). Nguyen-Quoc et al. (1999) observed that compared to the control the fruits of transgenic lines had a significant increase of 2.4-fold and $27 \%$ in SPS and SUSY activity respectively, no profound change in invertase activity, and only a slight decrease in ADP-glucose pyrosphosphorylase activity. In order to distinguish between the effects in leaves and fruits due to overexpression of transgene SPS, the authors also studied the sucrose unloading and turnover on the detached fruits using $\left[\mathrm{H}^{3}\right]$ (fructosyl)-sucrose. They observed an increase of $60 \%$ in sucrose turnover, which is extrapolated to an increase of $70 \%$ in sucrose import, in fruits of transformed plants relative to the control. Thus, it seems that the high activity of SPS and SUSY in fruits plays an important role in plant sink strength.

It is intriguing that some of the observations for plants with high sucrose metabolism and superior performance mentioned above are also found in high altitude wild accession $S$. arcanum LA385 and reported in this thesis (Chapter 2). These include similar values for total plant fresh weight, total plant dry weight, dry matter fraction to fruits, leaf sucrose-6phosphate and leaf sucrose contents, photosynthetic rates at low- and saturating-light, and 1.7 times increase in fruit number in LA385 plants at SOT compared to CT in week 16 after sowing (or 11 weeks after being transferred to SOT) at low light $\left(300 \mathrm{~mol} \mathrm{~m}^{-2} \mathrm{~s}^{-1}\right)$ and ambient $\left[\mathrm{CO}_{2}\right]$ of $400 \mathrm{ppm}$; whereas those parameters in $\mathrm{cv}$. Moneymaker are negatively affected by SOT. In another study, Kläring et al. (2015) investigated two tomato genotypes, i.e. a round type and a cherry type, grown in the greenhouses at ambient $\left[\mathrm{CO}_{2}\right]$ and at low temperatures $16 / 14^{\circ} \mathrm{C}$ or $11 / 9^{\circ} \mathrm{C}$ and compared that to the standard temperature $20 / 18^{\circ} \mathrm{C}$. Those authors observed both genotypes accumulate higher contents of soluble sugars and starch in leaves at lower temperatures compared to those at higher temperature. Yet those authors did not give detailed information about the contents of fructose, glucose, and sucrose separately. It is reported that the higher accumulation of those carbohydrates in leaves during the day is remobilized during the night. Those authors found no difference in total dry matter production and crop net photosynthesis between different growing temperatures. From these observations, it is concluded that feedback inhibition of photosynthesis by sugar and starch accumulation can be ruled out when cultivating fruit-bearing tomato plants at a low temperature. However, as mentioned earlier, the study of Kläring et al. (2015) and studies in this thesis (Chapter 2 and Chapter 4) were conducted at ambient $\left[\mathrm{CO}_{2}\right]$, while $\left[\mathrm{CO}_{2}\right]$ enrichment can be up to $1200 \mathrm{ppm}$ in commercial greenhouses in northern Europe. Therefore, it is still not known if that feedback inhibition mechanism is also absent when tomatoes are grown at low temperature and high $\left[\mathrm{CO}_{2}\right]$ in commercial greenhouses. 


\section{Natural variation in key players involved in sucrose metabolism offers a way to breed for low temperature tolerance in tomato}

Genetic variation is a valuable tool that has been used in plant and animal breeding to improve specific traits. For instance, the introgression of Lin5 gene encoding cell wall invertase from wild species $S$. pennellii LA716 into cultivated tomato background has been reported to increase fruit yield and fruit Brix value (Fridman et al. 2000, Fridman et al. 2002, Fridman et al. 2004). Wild species S. pimpinellifolium G1.1554 has also been used to introduce resistance against tomato yellow leaf curl virus (TYLCV) (Víquez-Zamora et al. 2014b). For abiotic stress such as low temperature in tomato, it is known that enzyme kinetics is reduced at low temperatures. Thus, in this project we aimed to explore the natural genetic resource to search for the variants in sucrose synthases (SUSYs) which can perform better than the variants in the cultivated tomatoes at low temperatures (Chapter 3). We found a large number of polymorphisms in the genomes of 32 accessions of related wild species compared to that of cv Moneymaker, which lead to amino acid changes in the peptide sequences of SUSY1, SUSY3, and SUSY4. We observed that most of the measured parameters, such as affinity toward substrate $\left(\mathrm{K}_{\mathrm{M}}\right)$, maximal velocity in catalyzing the substrate $\left(\mathrm{V}_{\max }\right)$, and catalytic efficiency $\left(\mathrm{V}_{\max } / \mathrm{K}_{\mathrm{M}}\right)$, in all the tested recombinant SUSY1 and SUSY3 variants were significantly reduced at low assay temperature $16^{\circ} \mathrm{C}$ compared to at control assay temperature $25^{\circ} \mathrm{C}$ (Table 4). However when comparing those parameters among three different variants, we observed a significant improvement in $\mathrm{V}_{\max } / \mathrm{K}_{\mathrm{M}}$ of SUSY3 of haplotype \#9 with the variations S53A, S106I, E727D, and K741E compared to that of haplotype\#1 (known as the control) at $16^{\circ} \mathrm{C}$. SUYS3 haplotype\#9 also showed slight improvement in all other parameters compared to SUSY3 haplotye\#1 at both assay temperatures. Interestingly, only the high altitude wild species $S$. arcanum LA385 and $S$. arcanum LA2172 have SUYS3 haplotype\#9, while cv Moneymaker (also known as $S$. lycopersicum LA2706) and another 42 genotypes have SUSY3 haplotype\#1 (S3 Table). Could the improved kinetics in SUSY3 of accession LA385, observed in vitro in Chapter 3, contribute to the higher sink strength and better acclimation to SOT in accession LA385 reported in Chapter 2? SUSY3 haplotype\#10 is another interesting allele that was studied for its enzyme kinetics in vitro at low temperature in Chapter 3. SUSY3 haplotype\#10 has an extra variation compared to haplotype\#9, i.e. Q349L, and is found in four accessions (S3 Table in Chapter 3). The only slight improvement in kinetics of the recombinant SUSY3 haplotype\#10 was that its $\mathrm{V}_{\max }$ value was not significantly reduced at $16^{\circ} \mathrm{C}$ compared to $25^{\circ} \mathrm{C}$ as observed in SUSY3 haplotype\#1 and haplotype\#9 (Table 4 in Chapter 3). The high altitude wild species S. neorickii G1.1601 (also known as LA0735) is one of the four accessions having SUSY3 haplotype\#10, and is the donor parent of an IL containing wild allele Susy3 (called Susy3-IL) in BC3S2 population (Chapter 4). The preliminary results in Chapter 4 showed that the Susy3-IL performed inferior to the recurrent parent $\mathrm{cv}$ Moneymaker especially at SOT, but had a slight improvement in dry matter allocation to fruits compared to that of $\mathrm{cv}$ Moneymaker at CT (Fig. 3). Is the linkage drag present in $\mathrm{BC} 3 \mathrm{~S} 2$ generation responsible for the inferior performance of Susy3-IL? Or is the inferior kinetics of SUSY3 of accession G1.1601 at low temperature, which was shown in Table 4 of Chapter 3, the actual cause for this observation? In addition, while the effects of SOT on 
growth and physiology of accession LA385 has been studied in Chapter 3, these effects are still unknown for G1.1601. Therefore, more studies are needed to understand better the performance of accession G1.1601 at low temperatures.

With the fast development of gene editing technologies (Khan 2019), there are new options to study and validate the function of the gene of interest in a more precise manner, and obtain novel products. Therefore, in future studies the effect in enzyme kinetics and plant performance at SOT caused by variation in SUSY3 can be investigated by using gene editing technologies in one of the following three options: 1. substitute SUSY3 by haplotype\#1 in LA385, or 2. substitute SUSY3 by haplotype\#9 in cv Moneymaker, or 3. substitute SUSY3 by haplotype\#9 in BC3S2 Susy3-IL. The positive role of SUSY3 haplotype\#9 in SOT tolerance will be better established in planta if: 1. gene-edited LA385 with SUSY3 haplotype\#1 has inferior performance at SOT compared to the non-edited control; 2. geneedited cv Moneymaker with SUSY3 haplotype \#9 acclimates to SOT better than the nonedited control; and 3. gene-edited Susy3-IL with SUSY3 haplotype\#9 has a superior performance at SOT compared to the non-edited control. However, the main challenge is how to make changes in four different and distant locations in the genome of tomato, so that the LA385 will have the SUSY3 haplotype\#1 instead of haplotype\#9 (option 1) or cv Monemaker will have the SUSY3 haplotype\#9 instead of haplotype\#1 (option 2). Although theoretically straightforward, up till now, we are not aware of any studies reporting such possible changes in one single event, even with the popular CRISPR-Cas9 (clustered regularly interspaced short palindromic repeats and CRISPR-associated protein 9) genome editing system. In a recent report, Anzalone et al. (2019) reported a new genome editing system called prime editing. This system makes use of a catalytically impaired Cas 9 endonuclease fused to an engineered reverse transcriptase, wired with a prime editing guide RNA that both specifies the target site and encodes the desired edit. The authors showed that prime editing is superior to CRISPR-Cas9 in several important aspects, in which it is: i. able to do targeted transversion (e.g. $\mathrm{C} \rightarrow \mathrm{A}, \mathrm{C} \rightarrow \mathrm{G}, \mathrm{G} \rightarrow \mathrm{C}, \mathrm{G} \rightarrow \mathrm{T}, \mathrm{A} \rightarrow \mathrm{C}, \mathrm{A} \rightarrow \mathrm{T}, \mathrm{T} \rightarrow \mathrm{A}$, and $\mathrm{T} \rightarrow \mathrm{G}$ ), ii. able to do three-base insertion or four-based deletion without making double-strand DNA breaks, iii. able to induce much lower off-target editing at known Cas9 off-target sites. This prime editing could be a potential tool to use for making the edits in the genome mentioned above, though it would need more plant generations to get the desired changes in four different distant positions in the genome. Thus, option 3 is the most feasible one to test with this prime editing tool because it needs only one editing event in the genome of the BC3S2 Susy3-IL with haplotype\#10 to cause the amino acid change from 349Q to 349L as in haplotype\#9. However one should know that the final products from gene-edited lines by such tool as CRISPR are currently deemed as genetic-modified organisms in Europe (Case C-528/16, The Court of Justice of the European Union) which will make practical use questionable.

Backcrossing the Susy3 allele from LA385 into elite cultivated tomato backgrounds is another approach to breed for new varieties that can perform well at SOT. In this approach, several steps need to be taken in order to obtain the advanced IL containing Susy3 allele from accession LA385. First, it is important to overcome the incompatibility barrier when crossing 
S. arcanum LA385 and cultivated tomato such as S. lycopersicum cv Moneymaker because interspecific hybridization between these two distant species has been reported to be prevented by both pre-zygotic and post-zygotic barriers (McGuire and Rick 1954). However, several studies have shown that such barriers can be overcome successfully by embryo rescue (Encina et al. 2012, Kharkongar et al. 2013, Sohrab et al. 2015). Subsequently, it is followed by backcrossing with the recurrent cultivated parent (e.g. cv Moneymaker) at least several generations to narrow down as much as possible the introgressed genome segment from LA385, which is also to reduce the unwanted effects from linkage drag. Individual plant selection in the backcrossing steps can be facilitated by the use of molecular markers, including both markers for the selection of desired changes and markers to prevent linkage drag. In this way, it is more cost effective because less space, time and man labor are needed to produce seeds in each backcross generation, which helps to speed up the breeding process.

\section{A solution to the challenge in testing the functions of the variants}

The cost for (re-)sequencing genomes has significantly decreased in the last two decades since the advent of next generation sequencing (NGS) technologies, for instance from $\$ 100$ million in 2001 down to approximately $\$ 1000$ per human genome in 2019 (National Human Genome Research Institute 2019). We now have the unprecedented ease to generate massive amounts of data via NGS technologies, which allow us conveniently to mine for genetic variations, as we have done in Chapter 3. However, it is another story to be able to go from the identification of genetic variations to the testing of function in all the identified variants. Freeman et al. (2011) have shown that the amino acid substitutions far away from the active site can affect the phosphatase activity of the enzyme phosphatase CheZ in bacteria Escherichia coli. Therefore, it is still valuable to evaluate the effects of amino acid substitutions in all the SUSY1/3/4 variants identified in Chapter 3 on enzyme kinetics, even though in silico analysis predicted that all the identified amino acid changes in SUSY1/3/4 are not close to the substrate binding and active sites of SUSY. As reported in Chapter 3, we selected a few variants for in depth characterization of the effect of the variations on the enzyme kinetics at different temperatures via conventional approach with several steps as following: 1. isolation of RNA of the target genes from the plants, 2. reverse transcription of the RNA into cDNA, 3. amplification and cloning of the specific Susy cDNA into intermediate plasmid, 4. transferring of the target into destination plasmid, 5. heterologous expression of the expression plasmid carrying Susy cDNA in a specific organism, e.g. budding yeast host lacking ability to hydrolyze sucrose in the growth medium, 6 . selection of the correct transformed clones based on the ability to break down sucrose in the medium for growth, 7. cultivation of the clones in larger scale to harvest cells, 8. purification of recombinant protein, 9. measurement of enzyme kinetics. This whole process is very time consuming and laborious, and only suitable for a few variants, e.g. two and three variants (haplotypes) for SUSY1 and SUSY3 in this thesis respectively. No in depth characterization has been made of other variants, namely 21, 19, and 17 haplotypes of SUSY1/3/4 respectively. In addition, we also identified 9, 5, and 1 variants in SUSY1/3/4 respectively in the mutagenized Micro-Tom population (Table 1). These were screened in 76 pooled samples, in which each pool contained 64 M2 individual plants derived from seed treatment 
with $1 \%$ EMS of $S$. lycopersicum cv. Micro-Tom. All these changes were different compared to those identified from the sequencing data of SUSY1/3/4 of 84 tomato accessions from The 150 Tomato Resequencing Project reported in Chapter 3. Even though all these induced amino acid alterations were also predicated not be close to putative substrate binding sites, they might still affect enzyme kinetics positively as shown in Chapter 3. Thus we might have missed even more variants potentially having improved kinetics at low temperatures.

Table 1: Summary of amino acid changes found in SUSY1/3/4 in the mutagenized Micro-Tom population in pooled samples

\begin{tabular}{l|l|l|l}
\hline Gene & $\begin{array}{l}\text { Number of } \\
\text { screened bp }\end{array}$ & $\begin{array}{l}\text { Nr of mutations leading } \\
\text { to amino acid change }\end{array}$ & Amino acid change \\
\hline \hline Susyl & 5221 & 9 & $\begin{array}{l}\text { P219L, T346I, A435V, G525E, A526T, } \\
\text { A526V, A526V, P533S, E699L }\end{array}$ \\
Susy3 & 5382 & 5 & R320H, P366S, R368K, D720N, S774F \\
Susy4 & 5448 & 1 & E373K \\
\hline \hline
\end{tabular}

This challenge of testing the functions of all the identified variants could be tackled by employing a higher throughput screening method known collectively as "deep mutational scanning" (Hietpas et al. 2011, Kim et al. 2013, Fowler and Fields 2014, Matuszewski et al. 2016). Deep mutational scanning links the performance of each protein variant to its encoding DNA in an expression plasmid via the DNA sequencing output (frequency) of each plasmid at the start (input libraries) and at the end of the growth selection (selection libraries). If to be used in our studied case for SUSYs, all 72 identified haplotypes from natural variation in Chapter 3 and the 15 extra haplotypes from the mutagenized Micro-Tom population could be assayed in vivo for their enzyme activity and kinetics at different temperatures in a high-throughput fashion. It is still necessary to go through steps 1 to 6 mentioned above, but subsequent steps are different. Step 7 will be simpler because we only need to grow smaller amounts of transformed yeast cells in micro-plates for plasmid isolation (step 8). After plasmid isolation, amplification of target regions are done with specific primers to prepare for sequencing (step 9), which will be followed by sequencing and analysis of sequencing data (step 10). The final output will be the ratio $R_{V} / R_{C}$, in which $R_{V}$ and $R_{C}$ are the sequencing reads from the variant and the control respectively. Therefore, the SUSY variants that are superior in breaking down sucrose in the growth medium will have higher ratio $R_{V} / R_{C}$ (Fig. 1). Since the costs of sequencing are lower now, this type of experiment becomes more feasible and offers a higher-throughput to validate the identified natural genetic variations in vivo. This experimental setup is also suitable for other classes of enzymes involved in sucrose metabolism in plants, e.g. invertases (sucrose hydrolysis) and sucrose transporters (e.g. SUT, SWEET). In this manner, we could study the effects of the identified natural genetic variation in enzyme kinetics in vivo at different conditions (e.g. 
temperatures) in higher throughput. This will help us obtain more insight about the natural variation and its effects on the function of genes of interest, which can be integrated into breeding programs to improve plant performance.

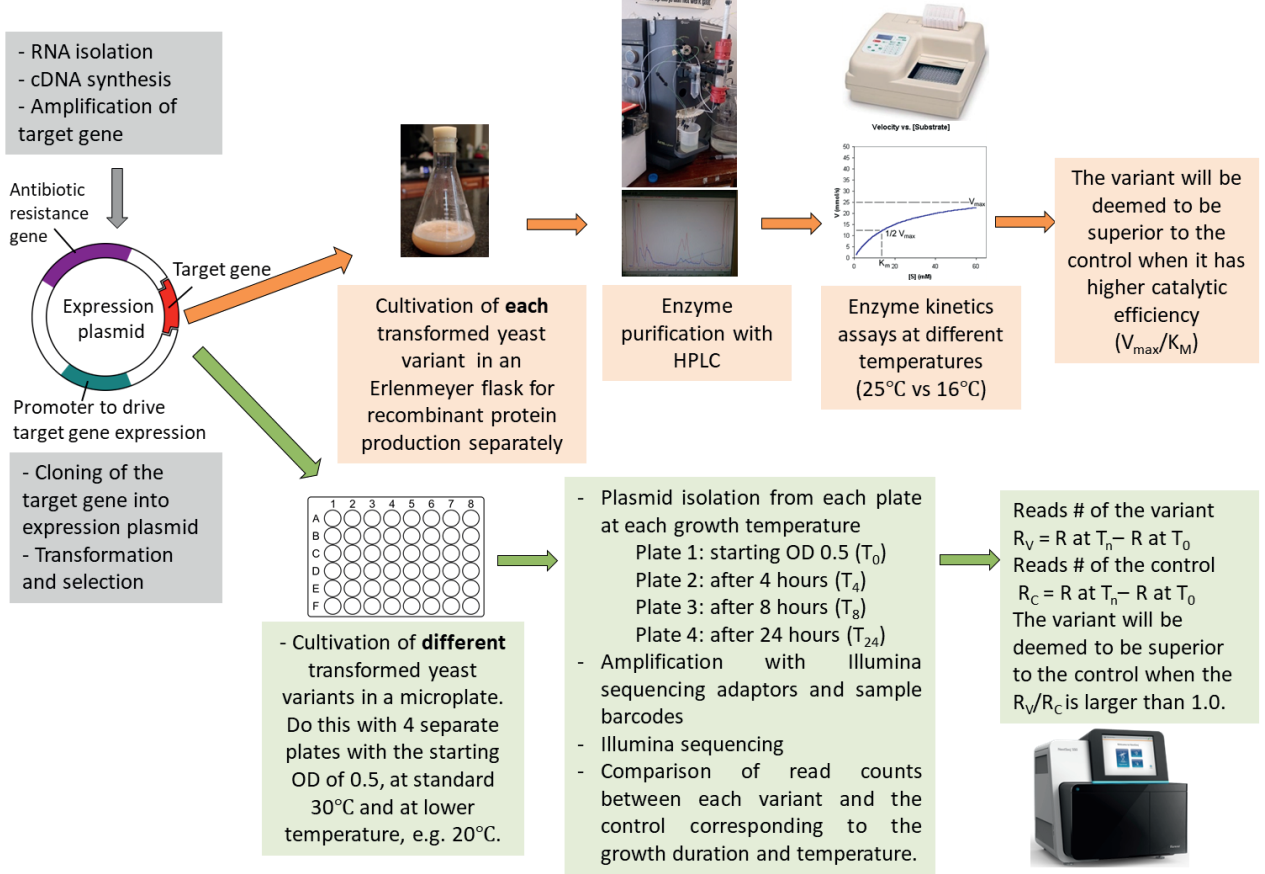

Figure 1. Two approaches to study effects of genetic variation on enzyme kinetics. The grey boxes are the common steps between two approaches. The light orange boxes are the approach used in Chapter 3, while the light green boxes are the alternative approach for high throughput. Images sources: carlschrammel.wordpress.com, bio-rad.com, illumina.com, and personal.

\section{Future for breeding for tomato as horticulture crop growing at sub-optimal temperature in a greenhouse}

Since greenhouse horticulture is an energy intensive sector in the Netherlands, innovations are needed to help this sector become more sustainable with lower energy consumption while keeping the plant yield and quality. The innovations should come from diverse aspects, from how the greenhouses are built, to which types of energy are used, to which types of plants are grown, and to how to maximize the use of the by-products and wastes (e.g. non-marketable parts of the tomato plants like leaves, stems, and roots) from the horticultural sector. For instance, a lot of efforts are going to the 2SaveEnergy concept - a consortium of horticultural experts involving the department of Greenhouse Horticulture of Wageningen University and Research (www.2saveenergy.nl). Several different types of greenhouses are being built with the latest technologies and materials in order to produce the most energy efficient greenhouse for each specific type of crop cultivation. As it is pointed out by this consortium, the innovations in energy-efficient designs should be based on three successive levels: 1. restrict 
energy consumption; 2. maximize use of sustainable sources of energy; and 3. have the most efficient use of fossil fuels for the remaining energy requirement. Recently Frames, a Dutch company with headquarters in Alphen aan den Rijn, has announced to be the first company in the world able to install a carbon neutral biomass system providing heating and supplying $\mathrm{CO}_{2}$ for industrial-scale greenhouse growers (Frames 2019). This is made possible by its own proprietary GALLOXOL ${ }^{\circledR}$ technology to capture $\mathrm{CO}_{2}$ from a biomass boiler. They claim that this biomass system can help the greenhouse growers save more than 6.5 million $\mathrm{m}^{3}$ of natural gas, and 12 million $\mathrm{kg}$ of fossil $\mathrm{CO}_{2}$ emissions per year. In addition, we believe that the development of crops that can grow well at lower temperatures without compromising the yield and quality will help the greenhouse horticulture sector to become more sustainable. The results described in this thesis have especially obtained more insight on the active sucrose metabolism in a wild species tomato, which appears to link to the ability to acclimate to lower growing temperature. In addition, the findings in this thesis have also shown that there is a treasure of natural variation to be exploited to boost up the sucrose metabolism in tomato grown at SOT. We are confident that with all the advances, exciting developments, and collaborations in different fields, e.g. recently started consortium of the Photosynthesis 2.0 program (WUR 2018), within ten years we will understand better the mechanism through which plants cope with SOT and will be able to breed for tomato cultivars performing as well at SOT as they do now at CT. 


\section{References}

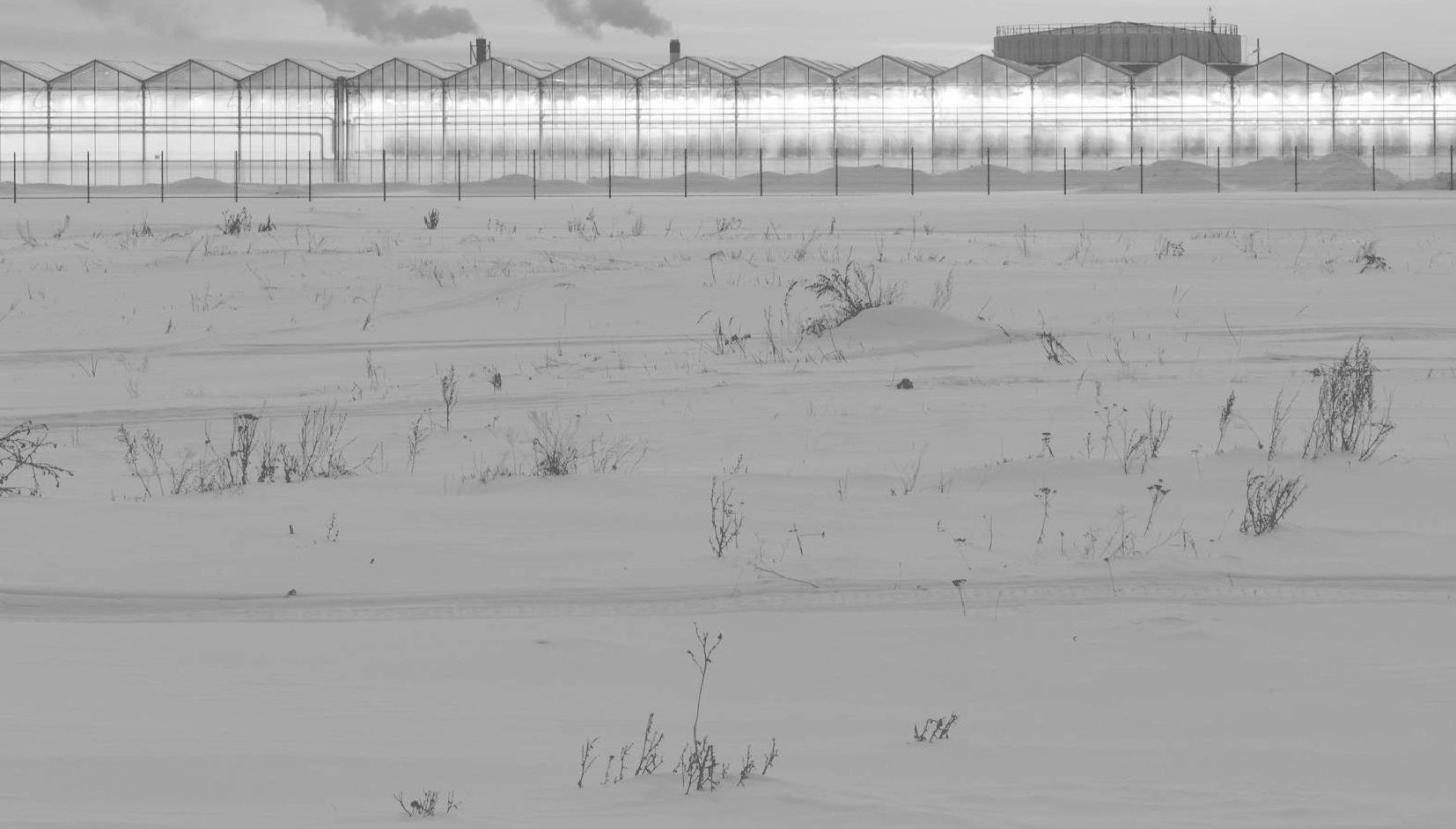


Adams, S. R., K. E. Cockshull and C. R. J. Cave (2001). "Effect of Temperature on the Growth and Development of Tomato Fruits." Annals of Botany 88(5): 869-877.

Aflitos, S., E. Schijlen, H. de Jong, D. de Ridder, S. Smit, R. Finkers, J. Wang, G. Zhang, N. Li, L. Mao, F. Bakker, R. Dirks, T. Breit, B. Gravendeel, H. Huits, D. Struss, R. Swanson-Wagner, H. van Leeuwen, R. C. H. J. van Ham, L. Fito, L. Guignier, M. Sevilla, P. Ellul, E. Ganko, A. Kapur, E. Reclus, B. de Geus, H. van de Geest, B. te Lintel Hekkert, J. van Haarst, L. Smits, A. Koops, G. Sanchez-Perez, A. W. van Heusden, R. Visser, Z. Quan, J. Min, L. Liao, X. Wang, G. Wang, Z. Yue, X. Yang, N. Xu, E. Schranz, E. Smets, R. Vos, J. Rauwerda, R. Ursem, C. Schuit, M. Kerns, J. van den Berg, W. Vriezen, A. Janssen, E. Datema, T. Jahrman, F. Moquet, J. Bonnet and S. Peters (2014). "Exploring genetic variation in the tomato (Solanum section Lycopersicon) clade by whole-genome sequencing." The Plant Journal 80(1): 136148.

Amor, Y., C. H. Haigler, S. Johnson, M. Wainscott and D. P. Delmer (1995). "A membraneassociated form of sucrose synthase and its potential role in synthesis of cellulose and callose in plants." Proceedings of the National Academy of Sciences of the United States of America 92(20): 9353-9357.

Anguenot, R., B. Nguyen-Quoc, S. Yelle and D. Michaud (2006). "Protein phosphorylation and membrane association of sucrose synthase in developing tomato fruit." Plant Physiology and Biochemistry 44(5-6): 294-300.

Anguenot, R., S. Yelle and B. Nguyen-Quoc (1999). "Purification of Tomato Sucrose Synthase Phosphorylated Isoforms by Fe(III)-Immobilized Metal Affinity Chromatography." Archives of Biochemistry and Biophysics 365(1): 163-169.

Anzalone, A. V., P. B. Randolph, J. R. Davis, A. A. Sousa, L. W. Koblan, J. M. Levy, P. J. Chen, C. Wilson, G. A. Newby, A. Raguram and D. R. Liu (2019). "Search-andreplace genome editing without double-strand breaks or donor DNA." Nature.

Appeldoorn, N. J. G., S. M. de Bruijn, E. A. M. Koot-Gronsveld, R. G. F. Visser, D. Vreugdenhil and L. H. W. van der Plas (1997). "Developmental changes of enzymes involved in conversion of sucrose to hexose-phosphate during early tuberisation of potato." Planta 202(2): 220-226.

Atkin, O. K., B. R. Loveys, L. J. Atkinson and T. L. Pons (2006a). "Phenotypic plasticity and growth temperature: understanding interspecific variability." J Exp Bot 57.

Atkin, O. K., B. R. Loveys, L. J. Atkinson and T. L. Pons (2006b). "Phenotypic plasticity and growth temperature: understanding interspecific variability." Journal of Experimental Botany 57(2): 267-281.

Bai, Y. and P. Lindhout (2007). "Domestication and Breeding of Tomatoes: What have We Gained and What Can We Gain in the Future?" Annals of Botany 100(5): 1085-1094.

Baker, N. R., J. Harbinson and D. M. Kramer (2007). "Determining the limitations and regulation of photosynthetic energy transduction in leaves." Plant, Cell \& Environment 30(9): 1107-1125.

Baroja-Fernández, E., F. J. Muñoz, J. Li, A. Bahaji, G. Almagro, M. Montero, E. Etxeberria, M. Hidalgo, M. T. Sesma and J. Pozueta-Romero (2012). "Sucrose synthase activity in the sus $1 /$ sus2/sus3/sus4 Arabidopsis mutant is sufficient to support normal cellulose and starch production." Proceedings of the National Academy of Sciences 109(1): 321-326.

Baroja-Fernández, E., F. J. Muñoz, M. Montero, E. Etxeberria, M. T. Sesma, M. Ovecka, A. Bahaji, I. Ezquer, J. Li, S. Prat and J. Pozueta-Romero (2009). "Enhancing Sucrose Synthase Activity in Transgenic Potato (Solanum tuberosum L.) Tubers Results in Increased Levels of Starch, ADPglucose and UDPglucose and Total Yield." Plant and Cell Physiology 50(9): 1651-1662. 
Barrantes, W., G. López-Casado, S. García-Martínez, A. Alonso, F. Rubio, J. J. Ruiz, R. Fernández-Muñoz, A. Granell and A. J. Monforte (2016). "Exploring New Alleles Involved in Tomato Fruit Quality in an Introgression Line Library of Solanum pimpinellifolium." Frontiers in plant science 7: 1172-1172.

Barratt, D. H. P., P. Derbyshire, K. Findlay, M. Pike, N. Wellner, J. Lunn, R. Feil, C. Simpson, A. J. Maule and A. M. Smith (2009). "Normal growth of Arabidopsis requires cytosolic invertase but not sucrose synthase." Proceedings of the National Academy of Sciences 106(31): 13124-13129.

Barrero-Sicilia, C., S. Hernando-Amado, P. González-Melendi and P. Carbonero (2011). "Structure, expression profile and subcellular localisation of four different sucrose synthase genes from barley." Planta 234(2): 391-403.

Bauchet, G. and M. Causse (2012). Genetic diversity in tomato (Solanum lycopersicum) and its wild relatives. Genetic diversity in plants, InTech.

Baud, S., M. N. Vaultier and C. Rochat (2004). "Structure and expression profile of the sucrose synthase multigene family in Arabidopsis." Journal of Experimental Botany 55(396): 397-409.

Bieniawska, Z., D. H. Paul Barratt, A. P. Garlick, V. Thole, N. J. Kruger, C. Martin, R. Zrenner and A. M. Smith (2007). "Analysis of the sucrose synthase gene family in Arabidopsis." The Plant Journal 49(5): 810-828.

Björkman, O. and B. Demmig (1987). "Photon yield of O2 evolution and chlorophyll fluorescence characteristics at $77 \mathrm{~K}$ among vascular plants of diverse origins." Planta 170(4): 489-504.

Blázquez, M. A., E. Santos, C.-1. Flores, J. M. Martínez-Zapater, J. Salinas and C. Gancedo (1998). "Isolation and molecular characterization of the Arabidopsis TPS1 gene, encoding trehalose-6-phosphate synthase." The Plant Journal 13(5): 685-689.

Bornke, F. and S. Sonnewald (2011). Biosynthesis and Metabolism of Starch and Sugars. Plant Metabolism and Biotechnology. H. Ashihara, A. Crozier and A. Komamine, John Wiley \& Sons, Ltd. .

Braun, D. M. (2012). "SWEET! The Pathway Is Complete." Science 335(6065): 173-174.

Braun, D. M., L. Wang and Y.-L. Ruan (2014). "Understanding and manipulating sucrose phloem loading, unloading, metabolism, and signalling to enhance crop yield and food security." Journal of Experimental Botany.

Brüggemann, W., T. W. Kooij and P. Hasselt (1992). "Long-term chilling of young tomato plants under low light and subsequent recovery. I. Growth, development and photosynthesis." Planta 186(2): 172-178.

Bürkle, L., J. M. Hibberd, W. P. Quick, C. Kühn, B. Hirner and W. B. Frommer (1998). "The $\mathrm{H}(+)$-Sucrose Cotransporter NtSUT1 Is Essential for Sugar Export from Tobacco Leaves." Plant Physiology 118(1): 59-68.

Cai, G., C. Faleri, C. Del Casino, A. M. C. Emons and M. Cresti (2011). "Distribution of Callose Synthase, Cellulose Synthase, and Sucrose Synthase in Tobacco Pollen Tube Is Controlled in Dissimilar Ways by Actin Filaments and Microtubules." Plant Physiology 155(3): 1169-1190.

Carlson, S. J., P. S. Chourey and P. S. Chourey (1996). "Evidence for plasma membraneassociated forms of sucrose synthase in maize." Molecular and General Genetics MGG 252(3): 303-310.

CBS. (2019, 29 March 2019). "Vegetables; yield and cultivated area per kind of vegetable." Retrieved 24 April, 2019, from https:/opendata.cbs.nl/statline/\#/CBS/en/dataset/37738ENG/table?dl=AB8B. 
Chen, A., S. He, F. Li, Z. Li, M. Ding, Q. Liu and J. Rong (2012). "Analyses of the sucrose synthase gene family in cotton: structure, phylogeny and expression patterns." BMC Plant Biology 12: 85-85.

Cheng, W.-H., E. W. Tallercio and P. S. Chourey (1996). "The Miniature1 Seed Locus of Maize Encodes a Cell Wall Invertase Required for Normal Development of Endosperm and Maternal Cells in the Pedicel." The Plant Cell 8(6): 971-983.

Chengappa, S., M. Guilleroux, W. Phillips and R. Shields (1999). "Transgenic tomato plants with decreased sucrose synthase are unaltered in starch and sugar accumulation in the fruit." Plant Molecular Biology 40(2): 213-221.

Chengappa, S., Loader, N. and Shields, R. (1998). "Cloning, expression and mapping of a second tomato (Lycopersicon esculentum) sucrose synthase gene, sus3 (accession Nos. AJ011319, AJ011543) (PGR 98-190)." Plant Physiology 118(4): 1533.

Chetelat, R. T., J. W. DeVerna and A. B. Bennett (1995). "Introgression into tomato (Lycopersicon esculentum) of the L. chmielewskii; sucrose accumulator gene (sucr) controlling fruit sugar composition." TAG Theoretical and Applied Genetics 91(2): 327-333.

Cho, J.-I., H.-B. Kim, C.-Y. Kim, T.-R. Hahn and J.-S. Jeon (2011). "Identification and Characterization of the Duplicate Rice Sucrose Synthase Genes OsSUS5 and OsSUS7 Which Are Associated with the Plasma Membrane." Molecules and Cells 31(6): 553561.

Choi, Y., G. Sims, S. Murphy, J. Miller and A. Chan (2012). "Predicting the Functional Effect of Amino Acid Substitutions and Indels." PLoS ONE 7(10).

Cid, E., R. R. Gomis, R. A. Geremia, J. J. Guinovart and J. C. Ferrer (2000). "Identification of Two Essential Glutamic Acid Residues in Glycogen Synthase." Journal of Biological Chemistry 275(43): 33614-33621.

Coleman, H. D., J. Yan and S. D. Mansfield (2009). "Sucrose synthase affects carbon partitioning to increase cellulose production and altered cell wall ultrastructure." Proceedings of the National Academy of Sciences 106(31): 13118-13123.

D'Aoust, M.-A., S. Yelle and B. Nguyen-Quoc (1999). "Antisense Inhibition of Tomato Fruit Sucrose Synthase Decreases Fruit Setting and the Sucrose Unloading Capacity of Young Fruit." The Plant Cell Online 11(12): 2407-2418.

de Bruijn, S. M., R. G. F. Visser and D. Vreugdenhil (1999). "Simultaneous analysis of a series of phosphorylated sugars in small tissue samples by anion exchange chromatography and pulsed amperometric detection." Phytochemical Analysis 10(3): 107-112.

De Coninck, B., K. Le Roy, I. Francis, S. Clerens, R. Vergauwen, A. M. Halliday, S. M. Smith, A. Van Laere and W. Van den Ende (2005). "Arabidopsis AtcwINV3 and 6 are not invertases but are fructan exohydrolases (FEHs) with different substrate specificities." Plant, Cell \& Environment 28(4): 432-443.

De Smet, K. A. L., A. Weston, I. N. Brown, D. B. Young and B. D. Robertson (2000). "Three pathways for trehalose biosynthesis in mycobacteria." Microbiology 146(1): 199-208.

Deeken, R., D. Geiger, J. Fromm, O. Koroleva, P. Ache, R. Langenfeld-Heyser, N. Sauer, S. T. May and R. Hedrich (2002). "Loss of the AKT2/3 potassium channel affects sugar loading into the phloem of Arabidopsis." Planta 216(2): 334-344.

Dillinger, J. (2017, 25-04-2017). "Largest Food Exports By Country." Retrieved 16-012019, 2019, from https:/www.worldatlas.com/articles/the-american-food-giant-thelargest-exporter-of-food-in-the-world.html.

Dinh, Q.-D., A. Dechesne, H. Furrer, G. Taylor, R. G. F. Visser, J. Harbinson and L. M. Trindade (2019). "High-Altitude Wild Species Solanum arcanum LA385-A 
Potential Source for Improvement of Plant Growth and Photosynthetic Performance at Suboptimal Temperatures." Frontiers in Plant Science 10(1163).

Dinh, Q.-D., R. Finkers, A. H. Westphal, W. M. A. M. van Dongen, R. G. F. Visser and L. M. Trindade (2018). "Exploring natural genetic variation in tomato sucrose synthases on the basis of increased kinetic properties." PLOS ONE 13(10): e0206636.

Dolman, M., G. Jukema and P. Ramaekers (2019). De Nederlandse landbouwexport 2018 in breder perspectief.

Duncan, K. A., S. C. Hardin and S. C. Huber (2006). "The Three Maize Sucrose Synthase Isoforms Differ in Distribution, Localization, and Phosphorylation." Plant and Cell Physiology 47(7): 959-971.

Duncan, K. A. and S. C. Huber (2007). "Sucrose Synthase Oligomerization and F-actin Association are Regulated by Sucrose Concentration and Phosphorylation." Plant and Cell Physiology 48(11): 1612-1623.

Echeverria, E., M. E. Salvucci, P. Gonzalez, G. Paris and G. Salerno (1997). "Physical and Kinetic Evidence for an Association between Sucrose-Phosphate Synthase and Sucrose-Phosphate Phosphatase." Plant Physiology 115(1): 223-227.

Elbein, A. D., Y. T. Pan, I. Pastuszak and D. Carroll (2003). "New insights on trehalose: a multifunctional molecule." Glycobiology 13(4): 17R-27R.

Elings, A., F. L. K. Kempkes, R. C. Kaarsemaker, M. N. A. Ruijs, N. J. van de Braak and T. A. Dueck (2005). "The Energy Balance and Energy-Saving Measures in Greenhouse Tomato Cultivation." Acta Horticulturae 619: 67-74.

Encina, C. L., E. Carmona-Martin and R. Fernández-Muñoz (2012). Embryo rescue of Solanum lycopersicum x S. peruvianum tomato cross for ToCV resistance, International Society for Horticultural Science (ISHS), Leuven, Belgium.

Eshed, Y. and D. Zamir (1995). "An Introgression Line Population of Lycopersicon pennellii in the Cultivated Tomato Enables the Identification and Fine Mapping of YieldAssociated QTL." Genetics 141(3): 1147-1162.

Everwilde Farms Inc. (2019). "Moneymaker heirloom tomato seeds." Retrieved 07-07-2019, 2019, from https://www.everwilde.com/store/Moneymaker-Heirloom-TomatoSeeds.html.

Finkers, R., Y. Bai, P. Berg, R. Berloo, F. Meijer-Dekens, A. Have, J. Kan, P. Lindhout and A. W. Heusden (2008). "Quantitative resistance to Botrytis cinerea from Solanum neorickii." Euphytica 159(1): 83-92.

Fiser, A. (2010). "Template-Based Protein Structure Modeling." Methods in molecular biology (Clifton, N.J.) 673: 73-94.

Flügge, U. I. and H. W. Heldt (1984). "The phosphate-triose phosphate-phosphoglycerate translocator of the chloroplast." Trends in Biochemical Sciences 9(12): 530-533.

Foolad, M. R. and G. Y. Lin (2000). "Relationship between cold tolerance during seed germination and vegetative growth in tomato: germplasm evaluation." Journal of the American Society for Horticultural Science 125(6): 679-683.

Fowler, D. M. and S. Fields (2014). "Deep mutational scanning: a new style of protein science." Nature Methods 11(8): 801-807.

Frames. (2019, 22 May 2019). "The world's first commercial $\mathrm{CO}_{2}$ capture installation for horticulture applications." Retrieved Nov 15, 2019, from http:/www.framesgroup.com/News-Events/First-commercial-CO2-capture-installation.

Freeman, A. M., B. M. Mole, R. E. Silversmith and R. B. Bourret (2011). "Action at a Distance: Amino Acid Substitutions That Affect Binding of the Phosphorylated CheY Response Regulator and Catalysis of Dephosphorylation Can Be Far from the CheZ Phosphatase Active Site." Journal of Bacteriology 193(18): 4709-4718. 
Fridman, E., F. Carrari, Y.-S. Liu, A. R. Fernie and D. Zamir (2004). "Zooming In on a Quantitative Trait for Tomato Yield Using Interspecific Introgressions." $\underline{\text { Science }}$ 305(5691): 1786-1789.

Fridman, E., Y. Liu, L. Carmel-Goren, A. Gur, M. Shoresh, T. Pleban, Y. Eshed and D. Zamir (2002). "Two tightly linked QTLs modify tomato sugar content via different physiological pathways." Molecular Genetics and Genomics 266(5): 821-826.

Fridman, E., T. Pleban and D. Zamir (2000). "A recombination hotspot delimits a wildspecies quantitative trait locus for tomato sugar content to $484 \mathrm{bp}$ within an invertase gene." Proceedings of the National Academy of Sciences 97(9): 4718-4723.

Fridman, E. and D. Zamir (2003). "Functional Divergence of a Syntenic Invertase Gene Family in Tomato, Potato, and Arabidopsis." Plant Physiology 131(2): 603-609.

Fujii, S., T. Hayashi and K. Mizuno (2010). "Sucrose Synthase is an Integral Component of the Cellulose Synthesis Machinery." Plant and Cell Physiology 51(2): 294-301.

Galtier, N., C. H. Foyer, J. Huber, T. A. Voelker and S. C. Huber (1993). "Effects of Elevated Sucrose-Phosphate Synthase Activity on Photosynthesis, Assimilate Partitioning, and Growth in Tomato (Lycopersicon esculentum var UC82B)." Plant physiology 101(2): 535-543.

Geigenberger, P. and M. Stitt (1993). "Sucrose synthase catalyses a readily reversible reaction in vivo in developing potato tubers and other plant tissues." Planta 189(3): 329-339.

Godt, D. E. and T. Roitsch (1997). "Regulation and Tissue-Specific Distribution of mRNAs for Three Extracellular Invertase Isoenzymes of Tomato Suggests an Important Function in Establishing and Maintaining Sink Metabolism." Plant Physiology 115(1): 273-282.

Goldschmidt, E. E. and S. C. Huber (1992). "Regulation of Photosynthesis by End-Product Accumulation in Leaves of Plants Storing Starch, Sucrose, and Hexose Sugars." Plant Physiology 99(4): 1443-1448.

Goren, S., S. Huber and D. Granot (2011). "Comparison of a novel tomato sucrose synthase, SISUS4, with previously described SISUS isoforms reveals distinct sequence features and differential expression patterns in association with stem maturation." Planta 233(5): 1011-1023.

Goren, S., N. Lugassi, O. Stein, Y. Yeselson, A. A. Schaffer, R. David-Schwartz and D. Granot (2017). "Suppression of sucrose synthase affects auxin signaling and leaf morphology in tomato." PLOS ONE 12(8): e0182334.

Goverment of the Netherlands. (2016, 28-04-2016). "Central government encourages sustainable energy." Retrieved 12-05-2019, from https://www.government.nl/topics/renewable-energy/central-government-encouragessustainable-energy.

Groen Links. (2018). "The Netherlands presents ambitious Climate Law." Retrieved 25 Sept, 2019, from https://groenlinks.nl/nieuws/netherlands-presents-ambitious-climatelaw.

Hardin, S. C., K. A. Duncan and S. C. Huber (2006). "Determination of Structural Requirements and Probable Regulatory Effectors for Membrane Association of Maize Sucrose Synthase 1." Plant Physiology 141(3): 1106-1119.

Hardin, S. C., G.-Q. Tang, A. Scholz, D. Holtgraewe, H. Winter and S. C. Huber (2003). "Phosphorylation of sucrose synthase at serine 170: occurrence and possible role as a signal for proteolysis." The Plant Journal 35(5): 588-603.

Hardin, S. C., H. Winter and S. C. Huber (2004a). "Phosphorylation of the Amino Terminus of Maize Sucrose Synthase in Relation to Membrane Association and Enzyme Activity." Plant Physiology 134(4): 1427-1438. 
Hardin, S. C., H. Winter and S. C. Huber (2004b). "Phosphorylation of the Amino Terminus of Maize Sucrose Synthase in Relation to Membrane Association and Enzyme Activity." Plant Physiology 134: 1427-1438.

Hemming, S. (2010). Energy and climate in Dutch greenhouses.

Heuvelink, E. (1995). "Effect of temperature on biomass allocation in tomato (Lycopersicon esculentum)." Physiologia Plantarum 94(3): 447-452.

Heuvelink, E. (2005). Tomatoes. Wallingford, CABI Publishing.

Heuvelink, E. and R. P. M. Buiskool (1995). "Influence of Sink-Source Interaction on Dry Matter Production in Tomato." Annals of Botany 75(4): 381-389.

Heuvelink, E. and M. Dorais (2005). Crop growth and yield. Tomatoes. E. Heuvelink. Wallingford, UK, CABI Publishing: 85-144.

Hietpas, R. T., J. D. Jensen and D. N. A. Bolon (2011). "Experimental illumination of a fitness landscape." Proceedings of the National Academy of Sciences 108(19): 78967901.

Ho, L. C. (1996). "The mechanism of assimilate partitioning and carbohydrate compartmentation in fruit in relation to the quality and yield of tomato." Journal of Experimental Botany 47(suppl 1): 1239.

Ho, L. C., R. I. Grange and A. J. Picken (1987). "An analysis of the accumulation of water and dry matter in tomato fruit." Plant, Cell \& Environment 10(2): 157-162.

Hoek, I. H. S., C. H. H. Ten Cate, C. J. Keijzer, J. H. Schel and H. J. M. Dons (1993). "Development of the Fifth Leaf is Indicative for Whole Plant Performance at Low Temperature in Tomato." Annals of Botany 72(4): 367-374.

Hohmann-Marriott, M. F. and R. E. Blankenship (2011). "Evolution of Photosynthesis." Annual Review of Plant Biology 62(1): 515-548.

HortiDaily. (2018, 12 Jan 2018). "Overview Global Tomato Market." Retrieved 25 Apr, 2019, from https://www.hortidaily.com/article/6040370/overview-global-tomatomarket/.

Horton, P., A. V. a. Ruban and R. G. Walters (1996). "REGULATION OF LIGHT HARVESTING IN GREEN PLANTS." Annual Review of Plant Physiology and Plant Molecular Biology 47(1): 655-684.

Huang, Y.-C., E.-C. Hsiang, C.-C. Yang and A.-Y. Wang (2016). "New insight into the catalytic properties of rice sucrose synthase." Plant Molecular Biology 90(1): 127135.

Hurd, R. G. and C. J. Graves (1985). "Some effects of air and root temperatures on the yield and quality of glasshouse tomatoes." Journal of Horticultural Science 60: 359-371.

Hurry, V. M., O. Keerberg, T. Pärnik, P. Gardeström and G. Öquist (1995). "Cold-hardening results in increased activity of enzymes involved in carbon metabolism in leaves of winter rye (Secale cereale L.)." Planta 195(4): 554-562.

Islam, M. S., T. Matsui and Y. Yoshida (1996). "Carbohydrate content and the activities of sucrose synthase, sucrose phosphate synthase and acid invertase in different tomato cultivars during fruit development." Scientia Horticulturae 65(2-3): 125-136.

Jia, L., B. Zhang, C. Mao, J. Li, Y. Wu, P. Wu and Z. Wu (2008). "OsCYT-INV1 for alkaline/neutral invertase is involved in root cell development and reproductivity in rice (Oryza sativa L.)." Planta 228(1): 51-59.

Jin, Y., D.-A. Ni and Y.-L. Ruan (2009). "Posttranslational Elevation of Cell Wall Invertase Activity by Silencing Its Inhibitor in Tomato Delays Leaf Senescence and Increases Seed Weight and Fruit Hexose Level." The Plant Cell 21(7): 2072-2089.

Kaur, S., A. K. Gupta, N. Kaur, J. S. Sandhu and S. K. Gupta (2009). "Antioxidative Enzymes and Sucrose Synthase Contribute to Cold Stress Tolerance in Chickpea." Journal of Agronomy and Crop Science 195(5): 393-397. 
Keller, F., M. Frehner and A. Wiemken (1988). "Sucrose Synthase, a Cytosolic Enzyme in Protoplasts of Jerusalem Artichoke Tubers (Helianthus tuberosus L.)." Plant Physiology 88: 239-241.

Kelley, L. A., S. Mezulis, C. M. Yates, M. N. Wass and M. J. E. Sternberg (2015). "The Phyre2 web portal for protein modeling, prediction and analysis." Nat. Protocols 10(6): 845-858.

Khan, S. H. (2019). "Genome-Editing Technologies: Concept, Pros, and Cons of Various Genome-Editing Techniques and Bioethical Concerns for Clinical Application." Molecular Therapy - Nucleic Acids 16: 326-334.

Kharkongar, H. P., V. K. Khanna, W. Tyagi, M. Rai and N. T. Meetei (2013). "Wide Hybridization and Embryo-Rescue for Crop Improvement in Solanum." Agrotechnology S11.

Kim, I., C. R. Miller, D. L. Young and S. Fields (2013). "High-throughput analysis of in vivo protein stability." Molecular \& cellular proteomics : MCP 12(11): 3370-3378.

Klann, E. M., R. T. Chetelat and A. B. Bennett (1993). "Expression of Acid Invertase Gene Controls Sugar Composition in Tomato (Lycopersicon) Fruit." Plant Physiology 103(3): 863-870.

Kläring, H.-P., Y. Klopotek, A. Krumbein and D. Schwarz (2015). "The effect of reducing the heating set point on the photosynthesis, growth, yield and fruit quality in greenhouse tomato production." Agricultural and Forest Meteorology 214-215(214215): 178-188.

Klopotek, Y. and H. P. Kläring (2014). "Accumulation and remobilisation of sugar and starch in the leaves of young tomato plants in response to temperature." $\underline{\text { Scientia }}$ Horticulturae 180: 262-267.

Knoblauch, M. and W. S. Peters (2010). "Münch, morphology, microfluidics - our structural problem with the phloem." Plant, Cell \& Environment 33(9): 1439-1452.

Knoblauch, M. and W. S. Peters (2013). "Long-distance translocation of photosynthates: a primer." Photosynthesis Research 117(1): 189-196.

Koch, K. (2004). "Sucrose metabolism: regulatory mechanisms and pivotal roles in sugar sensing and plant development." Current Opinion in Plant Biology 7(3): 235-246.

Kortstee, A., N. Appeldoorn, M. Oortwijn and R. Visser (2007). "Differences in regulation of carbohydrate metabolism during early fruit development between domesticated tomato and two wild relatives." Planta 226(4): 929-939.

Kromdijk, J., K. Głowacka, L. Leonelli, S. T. Gabilly, M. Iwai, K. K. Niyogi and S. P. Long (2016). "Improving photosynthesis and crop productivity by accelerating recovery from photoprotection." Science 354(6314): 857.

Labate, C. A. and R. C. Leegood (1988). "Limitation of photosynthesis by changes in temperature." Planta 173(4): 519-527.

Lammens, W., K. Le Roy, L. Schroeven, A. Van Laere, A. Rabijns and W. Van den Ende (2009). "Structural insights into glycoside hydrolase family 32 and 68 enzymes: functional implications." Journal of Experimental Botany 60(3): 727-740.

Lammens, W., K. Le Roy, A. Van Laere, A. Rabijns and W. Van den Ende (2008). "Crystal Structures of Arabidopsis thaliana Cell-Wall Invertase Mutants in Complex with Sucrose." Journal of Molecular Biology 377(2): 378-385.

Li, S., Y. Yang, Q. Zhang, N. Liu, Q. Xu and L. Hu (2018). "Differential physiological and metabolic response to low temperature in two zoysiagrass genotypes native to high and low latitude." PloS one 13(6): e0198885-e0198885.

Lunn, J. E., R. Feil, J. H. M. Hendriks, Y. Gibon, R. Morcuende, D. Osuna, W.-R. Scheible, P. Carillo, M.-R. Hajirezaei and M. Stitt (2006). "Sugar-induced increases in trehalose 6-phosphate are correlated with redox activation of ADPglucose pyrophosphorylase 
and higher rates of starch synthesis in Arabidopsis thaliana." The Biochemical journal 397(1): 139-148.

Ma, Y., Y. Zhang, J. Lu and H. Shao (2009). "Roles of plant soluble sugars and their responses to plant cold stress." African Journal of Biotechnology 8(10): 2004-2010.

Maloney, V. J., J.-Y. Park, F. Unda and S. D. Mansfield (2015). "Sucrose phosphate synthase and sucrose phosphate phosphatase interact in planta and promote plant growth and biomass accumulation." Journal of Experimental Botany 66(14): 4383-4394.

Marcelis, L. (1996). "Sink strength as a determinant of dry matter partitioning in the whole plant." Journal of Experimental Botany 47: 1281.

Marcelis, L. F. M. (1991). "Effects of Sink Demand on Photosynthesis in Cucumber." Journal of Experimental Botany 42(244): 1387-1392.

Mátrai, J., W. Lammens, A. Jonckheer, K. Le Roy, A. Rabijns, W. Van den Ende and M. De Maeyer (2008). "An alternate sucrose binding mode in the E203Q Arabidopsis invertase mutant: An X-ray crystallography and docking study." Proteins: Structure, Function, and Bioinformatics 71(2): 552-564.

Matuszewski, S., M. E. Hildebrandt, A.-H. Ghenu, J. D. Jensen and C. Bank (2016). "A Statistical Guide to the Design of Deep Mutational Scanning Experiments." Genetics 204(1): 77-87.

McGuire, D. C. and C. M. Rick (1954). "Self-incompatibility in species of Lycopersicon Sect. Eriopersicon and hybrids with L. esculentum." Hilgardia 23(4): 101-124.

Medek, D. E., M. C. Ball and M. Schortemeyer (2007). "Relative contributions of leaf area ratio and net assimilation rate to change in growth rate depend on growth temperature: comparative analysis of subantarctic and alpine grasses." New Phytologist 175(2): 290-300.

Meng, G. and K. Futterer (2003). "Structural framework of fructosyl transfer in Bacillus subtilis levansucrase." Nat Struct Mol Biol 10(11): 935-941.

Micallef, B. J., K. A. Haskins, P. J. Vanderveer, K.-S. Roh, C. K. Shewmaker and T. D. Sharkey (1995). "Altered photosynthesis, flowering, and fruiting in transgenic tomato plants that have an increased capacity for sucrose synthesis." Planta 196(2): 327-334.

Michelet, B. and M. Boutry (1995). "The plasma membrane H+-ATPase (A highly regulated enzyme with multiple physiological functions)." Plant Physiology 108(1): 1.

Miron, D., M. Petreikov, N. Carmi, S. Shen, I. Levin, D. Granot, E. Zamski and A. A. Schaffer (2002). "Sucrose uptake, invertase localization and gene expression in developing fruit of Lycopersicon esculentum and the sucrose-accumulating Lycopersicon hirsutum." Physiologia Plantarum 115(1): 35-47.

MONGABAY. (2008, 07-12-2008). "World Statistics [sorted by Area]." Retrieved 16-012019, 2019, from https://data.mongabay.com/igapo/world statistics by area.htm.

Morell, M. and L. Copeland (1985). "Sucrose Synthase of Soybean Nodules." Plant Physiology 78: 149-154.

Müller, P., X. P. Li and K. K. Niyogi (2001). "Non-photochemical quenching. A response to excess light energy." Plant physiology 125(4): 1558-1566.

Muszynska, G., L. Andersson and J. Porath (1986). "Selective adsorption of phosphoproteins on gel-immobilized ferric chelate." Biochemistry 25(22): 6850-6853.

Muszyńska, G., G. Dobrowolska, A. Medin, A. Medin, P. Ekman and J. O. Porath (1992). "Model studies on iron(III) ion affinity chromatography: II. Interaction of immobilized iron(III) ions with phosphorylated amino acids, peptides and proteins." Journal of Chromatography A 604(1): 19-28.

Nakai, T., N. Tonouchi, T. Konishi, Y. Kojima, T. Tsuchida, F. Yoshinaga, F. Sakai and T. Hayashi (1999). "Enhancement of cellulose production by expression of sucrose 
synthase in Acetobacter xylinum." Proceedings of the National Academy of Sciences of the United States of America 96(1): 14-18.

National Climate Assessment (2014). Extreme Weather.

National Human Genome Research Institute. (2019, 30 Oct 2019). "DNA Sequencing Costs: Data." Fact Sheets about Genomics Retrieved 01 Dec, 2019, from

https://www.genome.gov/about-genomics/fact-sheets/DNA-Sequencing-Costs-Data.

Nederhoff, E. M., A. N. M. De Koning and A. A. Rijsdijk (1992). "Leaf deformation and fruit production of glasshouse grown tomato (Lycopersicon esculentum Mill.) as affected by C02, plant density and pruning." Journal of Horticultural Science 67(3): 411-420.

Netherlands Foreign Investment Agency. (2017, Feburary 17, 2017). "The Netherlands has basically reinvented the tomato." Retrieved Dec 8, 2018, from

https://qz.com/907971/the-netherlands-basically-reinvented-the-tomato/.

Nguyen-Quoc, B., H. N'Tchobo, C. H. Foyer and S. Yelle (1999). "Overexpression of sucrose phosphate synthase increases sucrose unloading in transformed tomato fruit." Journal of Experimental Botany 50(335): 785-791.

Ntatsi, G., D. Savvas, V. Papasotiropoulos, A. Katsileros, R. M. Zrenner, D. K. Hincha, E. Zuther and D. Schwarz (2017). "Rootstock Sub-Optimal Temperature Tolerance Determines Transcriptomic Responses after Long-Term Root Cooling in Rootstocks and Scions of Grafted Tomato Plants." Frontiers in Plant Science 8: 911.

Patade, V. Y., H. Meena, A. Grover, S. M. Gupta and M. Nasim (2018). "Containment evaluation, cold tolerance and toxicity analysis in Osmotin transgenic tomato (Solanum lycopersicum L. cv. Pusa Ruby)." 3 Biotech 8(10): 410.

Paul, M. J. and T. K. Pellny (2003). "Carbon metabolite feedback regulation of leaf photosynthesis and development." J Exp Bot 54.

Pellny, T. K., O. Ghannoum, J. P. Conroy, H. Schluepmann, S. Smeekens, J. Andralojc, K. P. Krause, O. Goddijn and M. J. Paul (2004). "Genetic modification of photosynthesis with E. coli genes for trehalose synthesis." Plant Biotechnology Journal 2(1): 71-82.

Persia, D., G. Cai, C. Del Casino, C. Faleri, M. T. M. Willemse and M. Cresti (2008). "Sucrose Synthase Is Associated with the Cell Wall of Tobacco Pollen Tubes." Plant Physiology 147(4): 1603.

Pesaresi, P., C. Mizzotti, M. Colombo and S. Masiero (2014). "Genetic regulation and structural changes during tomato fruit development and ripening." Frontiers in Plant Science 5: 124

Picken, A. J. F. (1984). "A review of pollination and fruit set in the tomato (Lycopersicon esculentum Mill.)." Journal of Horticultural Science(59): 1-13.

Pilot, G., F. Gaymard, K. Mouline, I. Chérel and H. Sentenac (2003). "Regulated expression of Arabidopsis Shaker $\mathrm{K}+$ channel genes involved in $\mathrm{K}+$ uptake and distribution in the plant." Plant Molecular Biology 51(5): 773-787.

Pommerrenig, B., F. Ludewig, J. Cvetkovic, O. Trentmann, P. A. W. Klemens and H. E. Neuhaus (2018). "In Concert: Orchestrated Changes in Carbohydrate Homeostasis Are Critical for Plant Abiotic Stress Tolerance." Plant and Cell Physiology 59(7): 1290-1299.

Pyl, E.-T., M. Piques, A. Ivakov, W. Schulze, H. Ishihara, M. Stitt and R. Sulpice (2012). "Metabolism and Growth in Arabidopsis Depend on the Daytime Temperature but Are Temperature-Compensated against Cool Nights." The Plant Cell 24(6): 24432469.

Qin, G., Z. Zhu, W. Wang, J. Cai, Y. Chen, L. Li and S. Tian (2016). "A Tomato Vacuolar Invertase Inhibitor Mediates Sucrose Metabolism and Influences Fruit Ripening." Plant Physiology 172(3): 1596. 
Rashidi, B. and L. M. Trindade (2018). "Detailed biochemical and morphologic characteristics of the green microalga Neochloris oleoabundans cell wall." Algal Research 35: 152-159.

Renz, A., L. Merlo and M. Stitt (1993). "Partial purification from potato tubers of three fructokinases and three hexokinases which show differing organ and developmental specificity." Planta 190(2): 156-165.

Robinson, N. L., J. D. Hewitt and A. B. Bennett (1988). "Sink Metabolism in Tomato Fruit: I. Developmental Changes in Carbohydrate Metabolizing Enzymes." Plant Physiology 87(3): 727-730.

Rodríguez, G. R., S. Muños, C. Anderson, S.-C. Sim, A. Michel, M. Causse, B. B. M. Gardener, D. Francis and E. van der Knaap (2011). "Distribution of SUN, OVATE, LC, and FAS in the Tomato Germplasm and the Relationship to Fruit Shape Diversity." Plant Physiology 156(1): 275-285.

Roitsch, T. and M.-C. González (2004). "Function and regulation of plant invertases: sweet sensations." TRENDS in Plant Science 9(12): 606-613.

Römer, U., H. Schrader, N. Günther, N. Nettelstroth, W. B. Frommer and L. Elling (2004). "Expression, purification and characterization of recombinant sucrose synthase 1 from Solanum tuberosum L. for carbohydrate engineering." Journal of Biotechnology 107(2): 135-149.

Rosa, M., C. Prado, G. Podazza, R. Interdonato, J. A. González, M. Hilal and F. E. Prado (2009). "Soluble sugars: Metabolism, sensing and abiotic stress: A complex network in the life of plants." PSB 4(5): 388-393.

Ruan, Y.-L. (2014). "Sucrose Metabolism: Gateway to Diverse Carbon Use and Sugar Signaling." Annual Review of Plant Biology 65(1): 33-67.

Ruan, Y.-L., D. J. Llewellyn and R. T. Furbank (2003). "Suppression of Sucrose Synthase Gene Expression Represses Cotton Fiber Cell Initiation, Elongation, and Seed Development." The Plant Cell 15(4): 952.

Ruan, Y.-L. and J. W. Patrick (1995). "The cellular pathway of postphloem sugar transport in developing tomato fruit." Planta 196(3): 434-444.

Šali, A. and T. L. Blundell (1993). "Comparative Protein Modelling by Satisfaction of Spatial Restraints." Journal of Molecular Biology 234(3): 779-815.

Sauerzapfe, B., L. Engels and L. Elling (2008). "Broadening the biocatalytic properties of recombinant sucrose synthase 1 from potato (Solanum tuberosum L.) by expression in Escherichia coli and Saccharomyces cerevisiae." Enzyme and Microbial Technology 43(3): 289-296.

Savitch, L., G. Gray and N. A. Huner (1997). "Feedback-limited photosynthesis and regulation of sucrose-starch accumulation during cold acclimation and lowtemperature stress in a spring and winter wheat." Planta 201(1): 18-26.

Smirnova, J., A. R. Fernie and M. Steup (2015). Starch Degradation. Starch: Metabolism and Structure. Y. Nakamura. Tokyo, Springer Japan: 239-290.

Smith, A. M. and M. Stitt (2007). "Coordination of carbon supply and plant growth." Plant, Cell \& Environment 30(9): 1126-1149.

Smith, A. M., S. C. Zeeman and S. M. Smith (2005). "Starch degradation." Annual Review of Plant Biology 56(1): 73-98.

Sohrab, S. S., P. S. Bhattacharya, D. Rana, M. A. Kamal and M. K. Pande (2015). "Development of interspecific Solanum lycopersicum and screening for Tospovirus resistance." Saudi journal of biological sciences 22(6): 730-738.

Steinhauser, M.-C., D. Steinhauser, K. Koehl, F. Carrari, Y. Gibon, A. R. Fernie and M. Stitt (2010). "Enzyme Activity Profiles during Fruit Development in Tomato Cultivars and Solanum pennellii." Plant Physiology 153(1): 80-98. 
Stitt, M., I. Wilke, R. Feil and H. W. Heldt (1988). "Coarse control of sucrose-phosphate synthase in leaves: Alterations of the kinetic properties in response to the rate of photosynthesis and the accumulation of sucrose." Planta 174(2): 217-230.

Stommel, J. R. (1992). "Enzymic Components of Sucrose Accumulation in the Wild Tomato Species Lycopersicon peruvianum." Plant Physiology 99(1): 324-328.

Strand, Å., V. Hurry, P. Gustafsson and P. Gardeström (1997). "Development of Arabidopsis thaliana leaves at low temperatures releases the suppression of photosynthesis and photosynthetic gene expression despite the accumulation of soluble carbohydrates." The Plant Journal 12(3): 605-614.

Strand, Å., V. Hurry, S. Henkes, N. Huner, P. Gustafsson, P. Gardeström and M. Stitt (1999). "Acclimation of Arabidopsis Leaves Developing at Low Temperatures. Increasing Cytoplasmic Volume Accompanies Increased Activities of Enzymes in the Calvin Cycle and in the Sucrose-Biosynthesis Pathway." Plant Physiology 119(4): 13871398.

Sun, J., T. Loboda, S.-J. S. Sung and C. C. J. Black (1992). "Sucrose Synthase in Wild Tomato, Lycopersicon chmielewskii, and Tomato Fruit Sink Strength." Plant Physiology 98: 1163-1169.

Sun, J., J. Zhang, C. T. Larue and S. C. Huber (2011). "Decrease in leaf sucrose synthesis leads to increased leaf starch turnover and decreased RuBP regeneration-limited photosynthesis but not Rubisco-limited photosynthesis in Arabidopsis null mutants of SPSA1." Plant, Cell \& Environment 34(4): 592-604.

Tang, G.-Q., M. Lüscher and A. Sturm (1999). "Antisense Repression of Vacuolar and Cell Wall Invertase in Transgenic Carrot Alters Early Plant Development and Sucrose Partitioning." The Plant Cell Online 11(2): 177-190.

Taylor, S. H. and S. P. Long (2017). "Slow induction of photosynthesis on shade to sun transitions in wheat may cost at least $21 \%$ of productivity." Philosophical transactions of the Royal Society of London. Series B, Biological sciences 372(1730): 20160543.

Turgeon, R. and B. G. Ayre (2005). Pathways and Mechanisms of Phloem Loading. Vascular Transport in Plants. N. M. Holbrook and M. A. Zwieniecki. Burlington, Academic Press: 45-67.

UC Davis Tomato Genetics Resource Center Accessions Search, UC Davis Tomato Genetics Resource Center.

United Nations. (2017, 21-06-2017). "World population projected to reach 9.8 billion in 2050, and 11.2 billion in 2100." Retrieved 23-01-2019, 2019, from https://www.un.org/development/desa/en/news/population/world-populationprospects-2017.html.

Untergasser, A. (2008). "RNA Miniprep using CTAB" Untergasser's Lab." Retrieved 21-01, 2013, from http://www.untergasser.de/lab/protocols/miniprep rna ctab v1 0.htm.

Van Bel, A. J. E. (2003). "The phloem, a miracle of ingenuity." Plant, Cell \& Environment 26(1): 125-149.

van der Knijff, A., J. Benninga and C. Reijnders (2004). Energie in de glastuinbouw. Ontwikkelingen in de sector en op bedrijven t/m 2003., LEI: 65.

van der Ploeg, A. and E. Heuvelink (2005). "Influence of sub-optimal temperature on tomato growth and yield: a review." Journal of Horticultural Science \& Biotechnology 80(6): 652-659.

van der Ploeg, A., E. Heuvelink and J. H. Venema (2007a). "Wild relatives as a source for sub-optimal temperature tolerance in tomato." Acta Horticulturae 761: 127-133.

van der Ploeg, A., M. van der Meer and E. Heuvelink (2007b). "Breeding for a more energy efficient greenhouse tomato: past and future perspectives." Euphytica 158(1): 129138. 
van der Velden, N. and P. Smit (2018). Energiemonitor van de Nederlandse glastuinbouw 2017, LEI: 64.

Vargas, W. A., H. G. Pontis and G. L. Salerno (2008). "New insights on sucrose metabolism: evidence for an active $\mathrm{A} / \mathrm{N}-\mathrm{Inv}$ in chloroplasts uncovers a novel component of the intracellular carbon trafficking." Planta 227(4): 795-807.

Venema, J. H., P. Linger, A. W. van Heusden, P. R. van Hasselt and W. Brüggemann (2005). "The Inheritance of Chilling Tolerance in Tomato (Lycopersicon spp.)." Plant biol (Stuttg) 7(EFirst): 118,130.

Venema, J. H., F. Posthumus, M. De Vries and P. R. Van Hasselt (1999a). "Differential response of domestic and wild Lycopersicon species to chilling under low light: growth, carbohydrate content, photosynthesis and the xanthophyll cycle." Physiologia Plantarum 105(1): 81-88.

Venema, J. H., F. Posthumus and P. R. van Hasselt (1999b). "Impact of Suboptimal Temperature on Growth, Photosynthesis, Leaf Pigments, and Carbohydrates of Domestic and High-altitude Wild Lycopersicon Species." Journal of Plant Physiology 155: 711-718.

Verhaest, M., W. Lammens, K. Le Roy, B. De Coninck, C. J. De Ranter, A. Van Laere, W. Van den Ende and A. Rabijns (2006). "X-ray diffraction structure of a cell-wall invertase from Arabidopsis thaliana." Acta Crystallographica Section D 62(12): 15551563.

Víquez-Zamora, M., M. Caro, R. Finkers, Y. Tikunov, A. Bovy, R. G. Visser, Y. Bai and S. van Heusden (2014a). "Mapping in the era of sequencing: high density genotyping and its application for mapping TYLCV resistance in Solanum pimpinellifolium." BMC Genomics 15(1): 1152.

Víquez-Zamora, M., M. Caro, R. Finkers, Y. Tikunov, A. Bovy, R. G. F. Visser, Y. Bai and S. van Heusden (2014b). "Mapping in the era of sequencing: high density genotyping and its application for mapping TYLCV resistance in Solanum pimpinellifolium." BMC Genomics 15(1): 1152.

Vogel, G., R. A. Aeschbacher, J. Müller, T. Boller and A. Wiemken (1998). "Trehalose-6phosphate phosphatases from Arabidopsis thaliana: identification by functional complementation of the yeast tps2 mutant." The Plant Journal 13(5): 673-683.

Volpicella, M., I. Fanizza, C. Leoni, A. Gadaleta, D. Nigro, B. Gattulli, G. Mangini, A. Blanco and L. R. Ceci (2016). "Identification and Characterization of the Sucrose Synthase 2 Gene (Sus2) in Durum Wheat." Frontiers in Plant Science 7(266).

Voorrips, R. E., W. Verkerke, R. Finkers, R. Jongerius and J. Kanne (2000). "Inheritance of taste components in tomato." Acta Physiologiae Plantarum 22(3): 259-261.

Wageningen UR. (2008). "Cetre for Photosyntehsis Research, 'Towards BioSolar Cells'." Retrieved 26-10, 2011, from http://documents.plant.wur.nl/pri/biosolar-safe.pdf.

Wang, E., J. Wang, X. Zhu, W. Hao, L. Wang, Q. Li, L. Zhang, W. He, B. Lu, H. Lin, H. Ma, G. Zhang and Z. He (2008). "Control of rice grain-filling and yield by a gene with a potential signature of domestication." Nature Genetics 40: 1370.

Wang, F., A. Sanz, M. L. Brenner and A. Smith (1993a). "Sucrose Synthase, Starch Accumulation, and Tomato Fruit Sink Strength." Plant Physiology 101(1): 321-327.

Wang, F., A. G. Smith and M. L. Brenner (1993b). "Isolation and Sequencing of Tomato Fruit Sucrose Synthase cDNA." Plant Physiology 103(4): 1463-1464.

Wang, F., A. G. Smith and M. L. Brenner (1994). "Temporal and Spatial Expression Pattern of Sucrose Synthase during Tomato Fruit Development." Plant Physiology 104(2): 535-540. 
Winter, H. and S. C. Huber (2000). "Regulation of Sucrose Metabolism in Higher Plants: Localization and Regulation of Activity of Key Enzymes." Critical Reviews in Biochemistry and Molecular Biology 35(4): 253-289.

Winter, H., J. L. Huber and S. C. Huber (1998). "Identification of sucrose synthase as an actin-binding protein." FEBS Letters 430(3): 205-208.

Worrell, A. C., J. M. Bruneau, K. Summerfelt, M. Boersig and T. A. Voelker (1991). "Expression of a maize sucrose phosphate synthase in tomato alters leaf carbohydrate partitioning." The Plant Cell 3(10): 1121-1130.

Wu, R., M. D. Asención Diez, C. M. Figueroa, M. Machtey, A. A. Iglesias, M. A. Ballicora and D. Liu (2015). "The Crystal Structure of Nitrosomonas europaea Sucrose Synthase Reveals Critical Conformational Changes and Insights into Sucrose Metabolism in Prokaryotes." Journal of Bacteriology 197(17): 2734-2746.

WUR. (2018). "Consortium of the Photosynthesis 2.0 program." Retrieved 19 April, 2020, from https://www.wur.nl/en/Research-Results/Themes/From-hunger-to-foodsecurity/photosynthesis/Consortium.htm.

WUR and Kas als Energiebron (2016). Further reductions in CO2 emissions in the greenhouse horticulture sector in 2016, WUR \& Kas als energiebron.

Xu, D.-P., S.-J. S. Sung, T. Loboda, P. P. Kormanik and C. C. Black (1989). "Characterization of Sucrolysis via the Uridine Diphosphate and PyrophosphateDependent Sucrose Synthase Pathway." Plant Physiology 90(2): 635-642.

Xu, X., R. G. F. Visser and L. M. Trindade (2014). Chapter 4 - Starch Modification by Biotechnology: State of Art and Perspectives A2 - Halley, Peter J. Starch Polymers. L. Avérous. Amsterdam, Elsevier: 79-102.

Yelle, S., J. D. Hewitt, N. L. Robinson, S. Damon and A. B. Bennett (1988). "Sink Metabolism in Tomato Fruit: III. Analysis of Carbohydrate Assimilation in a Wild Species." Plant Physiology 87(3): 737-740.

Zhang, L.-Y., Y.-B. Peng, S. Pelleschi-Travier, Y. Fan, Y.-F. Lu, Y.-M. Lu, X.-P. Gao, Y.-Y. Shen, S. Delrot and D.-P. Zhang (2004). "Evidence for Apoplasmic Phloem Unloading in Developing Apple Fruit." Plant Physiology 135(1): 574-586.

Zheng, Y., S. Anderson, Y. Zhang and R. M. Garavito (2011). "The Structure of Sucrose Synthase-1 from Arabidopsis thaliana and Its Functional Implications." Journal of Biological Chemistry 286(41): 36108-36118.

Zinn, K. E., M. Tunc-Ozdemir and J. F. Harper (2010). "Temperature stress and plant sexual reproduction: uncovering the weakest links." Journal of Experimental Botany 61(7): 1959-1968.

Zrenner, R., M. Salanoubat, L. Willmitzer and U. Sonnewald (1995). "Evidence of the crucial role of sucrose synthase for sink strength using transgenic potato plants (Solanum tuberosum L.)." The Plant Journal 7(1): 97-107.

Zrenner, R., K. Schüler and U. Sonnewald (1996). "Soluble acid invertase determines the hexose-to-sucrose ratio in cold-stored potato tubers." Planta 198(2): 246-252. 


\section{Summary}
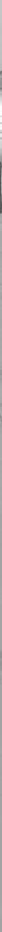


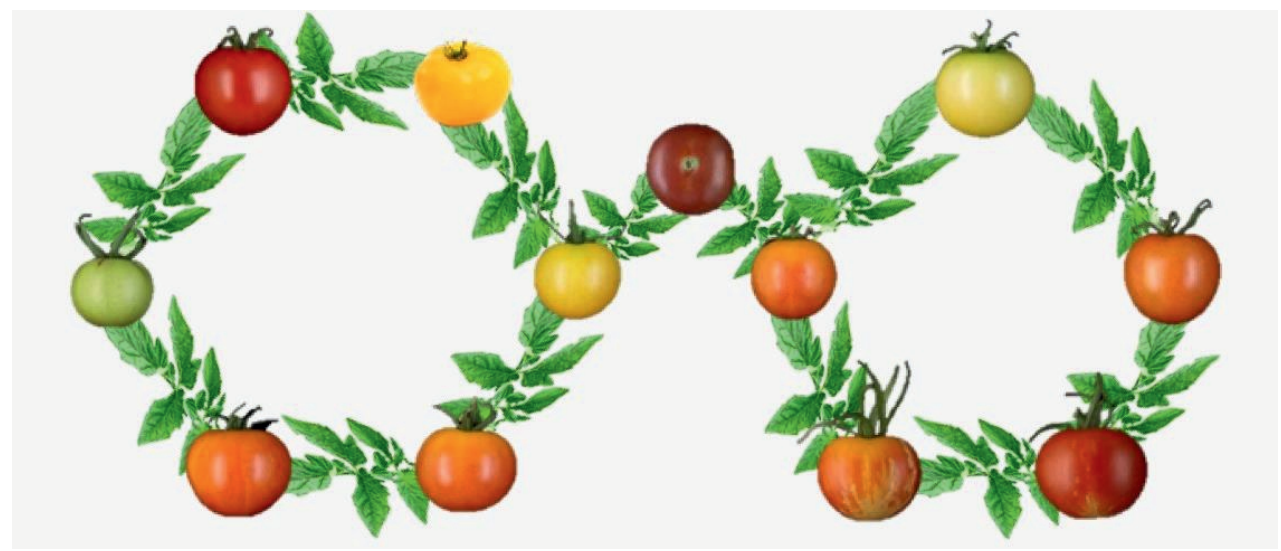

(A sucrose molecule displayed creatively with a variation of tomato fruit types and leaves. Design credit: Ernest Aliche)

Tomato is one of the most important vegetable crops in the world. The Netherlands, despite of its relatively small land area, is a leading player in producing and exporting tomato at a global scale. Due to the temperate climate in the Netherlands, the cultivation of tomato, as well as other major vegetables such as sweet pepper and cucumber, is mostly done in greenhouses. In this manner, the growth conditions, such as temperature, lighting, $\mathrm{CO}_{2}$ enrichment, humidity, can be maintained optimally in order to maximize yield of harvestable parts of the crops, i.e. fruits in this case. This type of cultivation in temperate climate areas requires very much energy, mainly for heating which take up $20-30 \%$ of the total production costs. This high energy consumption for cultivation is deemed to have also a high impact on the environment. Therefore, many efforts have been put into finding solutions to decrease the energy consumption in the greenhouse horticultural sector. For instance, several companies and research institutes have been looking into ways to make novel building materials, optimize light types and intensities to improve photosynthesis efficiency, develop new greenhouse architectures that can become energy-neutral in the future. In this thesis, we looked into the biological side of this issue, in which we set out to understand more about how tomato copes with sub-optimal temperatures (SOT), and explore the genetic variation that can be used to breed new tomato cultivars that perform well at SOT. SOT in this thesis is defined as the temperature below the current economical optimum growth temperature in heated greenhouses in the Netherlands of $19-20^{\circ} \mathrm{C}$ and above the chilling temperature of $12^{\circ} \mathrm{C}$.

In Chapter 1 I presented an introduction about why it is important to develop resilient crops for a sustainable economy in the current situation where the climate changes can have negative impact on food production. The importance of photosynthesis and sucrose metabolism as the direct downstream process on crop growth, development, and yield is discussed. Furthermore, we described in detail sucrose synthases and invertases - two classes of enzymes that directly hydrolyze sucrose molecules, and play a key role in sugar metabolism and transport. As the Netherlands has a temperate climate, reducing the growth 
temperatures in the greenhouses, i.e. SOT, is one of the options to bring down the energy consumption when cultivating crops with higher growth temperature than ambient temperature. However several studies have shown that plant growth and yield are negatively affected at SOT. I propose that key players in sucrose metabolism should be exploited to help plants to improve the sink strength, plant growth and yield at SOT.

In Chapter 2 we studied the impact of SOT (16/14C $\mathrm{C}^{\circ}$ day/night temperatures), in comparison to control temperature $\left(\mathrm{CT}, 22 / 20 \mathrm{C}^{\circ}\right)$, on plant growth, photosynthetic capacity, and carbohydrate metabolism in a cultivated tomato variety (Solanum lycopersicum cv Moneymaker) and a high altitude wild species tomato ( $S$. arcanum LA385). The study was performed on plants from flowering onset until a later stage of fruit development, in which tomato plants are known to be more sensitive to SOT. We observed that LA385 acclimated well to SOT at the later stage of fruit development in which there were no significant differences in plant growth, photosynthetic capacity, and carbohydrate metabolism, whereas cv Moneymaker was greatly affected by SOT in all the studied parameters. One of the striking findings was the highly active sucrose metabolism in leaf and fruit tissues of LA385 in comparison to that in cv Moneymaker. Overall, the findings in this chapter indicated that LA385 is a potential candidate to be used in a breeding program for new cultivars that can perform well at SOT.

In Chapter 3 we explored the genetic variation in three alleles Susyl/3/4 encoding for sucrose synthase (SUSY). Sucrose synthase is one of the two enzymes directly hydrolyzing sucrose in plants, and deemed to play an important role in sucrose metabolism and plant sink strength. The panel included 84 tomato accessions in total, in which 53 accessions are cultivated tomato and landraces, and 32 accessions are related wild species. As expected, we found fewer variations in Susyl/3/4 in the cultivated and landraces tomatoes, but much more in related wild species. The variation in the deduced amino acid sequences was grouped into 23, 22, and 17 distinct haplotypes for SUSY1/3/4, respectively. Strikingly, in silico analysis of the variations showed that all the known substrate binding sites were highly conserved. Because it has been shown that amino acid changes outside the substrate binding sites can still have a positive effect on enzyme kinetics, we continued with cloning and heterologous expression of a few haplotypes of SUSY1 and SUSY3. Amongst the studied haplotypes, SUSY-haplotype\#9, with four alterations, S53A, S106I, E727D, and K741E found in $S$. arcanum LA385 (the same accession as studied in Chapter 2) and S. arcanum LA2172, showed an improved catalytic efficiency $\left(\mathrm{V}_{\max } / \mathrm{K}_{\mathrm{m}}\right)$ at $16 \mathrm{C}^{\circ}$ compared to the reference SUSYhaplotype\#1 found in cv Moneymaker for instance. The findings in this chapter demonstrate that SUSY kinetic properties can be enhanced by exploiting natural variation, and the potential of this enzyme to improve sucrose metabolism and eventually sink strength in planta at SOT.

In Chapter 3, we found five amino acid differences S53A, S106I, Q349L, E727D, and K741E in SUSY3 in the high altitude wild species S. neorickii G1.1601 (SUSY3haplotype\#10) compared to that of the reference SUSY3-haplotype\#1 in cv Moneymaker. This SUSY3-haplotype\#10 has only one alteration Q349L compared to that of SUSY3haplotype\#9 found in $S$. arcanum LA385. Since we had a BC3S2 introgression line called 
Susy3-IL in house which has been identified to carry Susy3 allele of S. neorickii G1.1601, we studied the impact of SOT $\left(16 / 14 \mathrm{C}^{\circ}\right)$ in comparison to control temperature $\left(\mathrm{CT}, 25 / 16 \mathrm{C}^{\circ}\right)$ on plant growth, carbohydrate metabolism, gene expression and enzyme activity of the key enzymes sucrose synthase and invertase in this Susy3-IL in comparison to the recurrent parent tomato $S$. lycopersicum cv Moneymaker in Chapter 4. The study in Chapter 4 was performed on plants from flowering onset until a later stage of fruit development, in which tomato plants are known to be more sensitive to SOT. Overall Susy3-IL showed an inferior performance relatively to cv Moneymaker in most of the studied parameters at both CT and SOT. The only positive improvement that we observed was that Susy3-IL had a $9 \%$ increase in dry mater allocation to fruits compared to that in $\mathrm{cv}$ Moneymaker during the fruit ripening phase at CT. However due to the lack of biological replicates, we would need to confirm these results in future study in order to substantiate our initial findings.

In Chapter 5 I discussed all the findings presented in this thesis. I also integrated the findings with the current developments in different research fields, and proposed some new options to extend and exploit the results. I believe that with all the advances in research and development in different fields, and an increase in the interdisciplinary research in this particular field our understanding on how plants respond to SOT will be greatly improved. This will make it possible to screen and integrate the valuable natural variation into current germplasm more efficiently, so that we can eventually breed for new varieties that are more resilient to different sub-optimal growth conditions. 


\section{Acknowledgments}

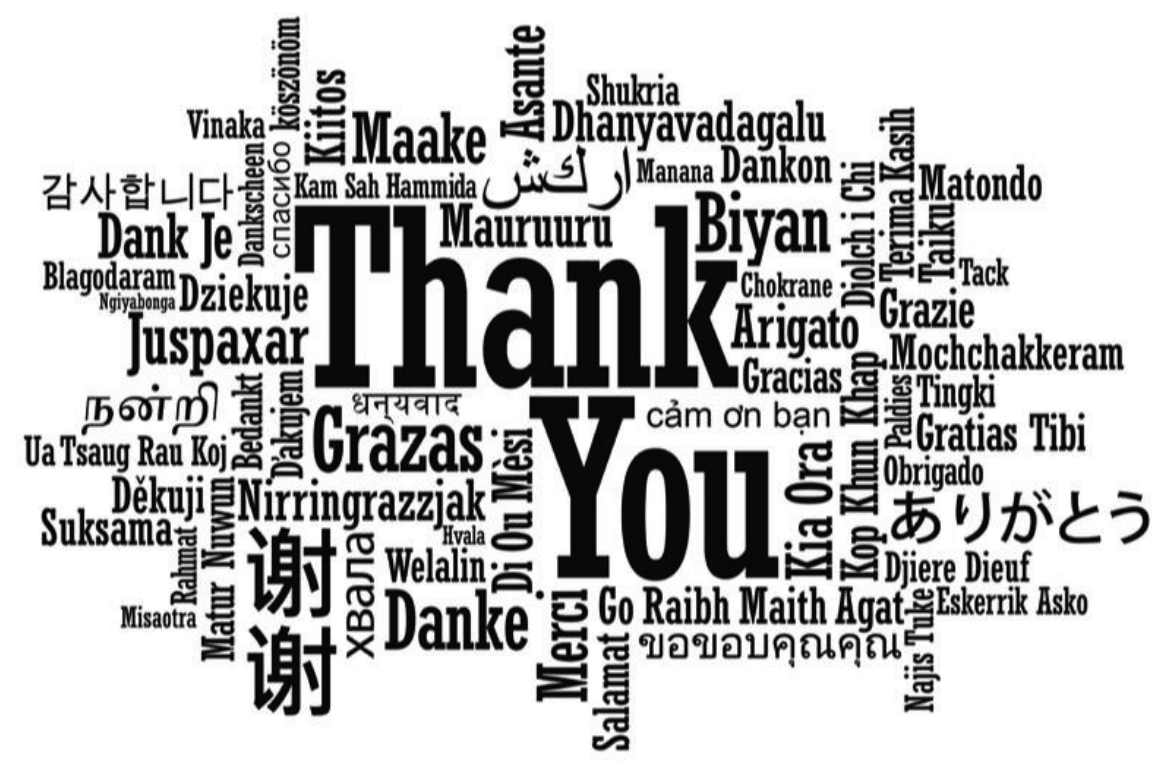


Time does fly as people say. As I look back to the period when I was on the go to learn and explore different aspects of this $\mathrm{PhD}$ project, so many memories flush back even though it was about 4 years ago that I left Plant Breeding department in June 2016. It's been a long journey, longer than I expected, in almost 9 years, but I did enjoy it and learn so much. Thankfully I have not gone through this journey alone, otherwise I would not have made it. There are so many people involving and supporting me in many different ways during all these 9 years that I would like to acknowledge here. It is going to be a long writing, so please be patient with me $:$.

First of all, I would like to express my deepest gratitude to my supervisor and promotor Luisa Trindade. Luisa, thank you for giving this opportunity to do this $\mathrm{PhD}$ project and guiding me these many years! When I got quite tired from the side effect of the medication, you quickly arrange things for me so that I could take more time to rest. So many times I have this feeling that I would never be able to finish this, but you kept encouraging and supporting me through all these many years. Despite of several set-backs in the projects, you always could find ways to help me steer through them, and to reach the goals. Thank you for your kindness and patience toward me especially during the writing period, which took like forever. Thank you for your constructive feedback, so that I could grow more in my research and writing skills. Aside from this, I also really enjoyed the monthly BioBased Economy (BBE) research group lunch that you initiated, in which we took turn to shop for or prepare home-made food to share with each other. And of course I and the whole group also enjoyed a lot the BBQ at your house! It was such as nice way to make the members of the group get to know each other more. I really admire your passion with your work, and the way you connect with people! In addition, I and Yuni also appreciate you, Rob and Lieke a lot for opening up your house, sharing with us nice meals and talks and also the visits that you gave us in both Wageningen and Almere, we are really grateful for it because we get to know you and your family more at personal level outside work. Secondly, I also want to express my gratefulness to my second promotor Richard Visser. Thank you Richard for always being very supportive and responsive to development of my $\mathrm{PhD}$ project. Despite of your busy schedule with many roles in WUR, you always managed to provide constructive advices and helped me stay on track. Whenever I need the feedback on the manuscripts, you always responded to it very swiftly. I also enjoyed the fact that you encouraged the activities in our chair group Plant Breeding (PBR), in which we could organize and enjoy together. They were very nice to build the connection and understanding among the colleagues, thank you Richard! I also would like to thank my supervisor Anne Kortstee. Thank you for guiding me in the first 2 years while you were in PBR WUR. It was not very smooth process as we hoped for at first, but I'm glad that we managed to sort things out. Yuni and I also enjoyed the time that you and your family came to visit and enjoyed a meal with us. Furthermore, I am also thankful to my external supervisor Richard Immink. I had a privilege to work for Richard and Susan Richard's PhD student when I was doing my bachelor internship and also as a research assistant after that at Plant Research International. Richard, thank you for following up on my $\mathrm{PhD}$ progress, for giving me valuable advice, as well as for connecting me to Biochemistry department for the purification of the recombinant proteins. I appreciate that you always keep 
encouraging me to push till the finishing line when we were talking on line as well as meeting on the open market in Wageningen during weekends.

Doing $\mathrm{PhD}$ projects require many lab works and greenhouse work. Thankfully I have had many practical and expert supports from many colleagues that I would like to express my gratitude. I want to thank especially: Heleen and Annemarie from BBE research group of PBR WUR with the help in the biochemistry lab; Richard Finkers of PBR WUR for help with bioinformatics part of the tomato genome projects; Walter and Adrie from Biochemistry department for the extraction and purification of recombinant proteins; Jeremy and Taylor from Horticulture and Product Physiology Group with the help of photosynthetic measurements. To Heleen, besides works, you are one of my favorite colleagues. I always enjoyed hearing your adventurous trips with your husband, and hope that I can do such trips with Yuni somedays. You also took time to listen to my stories and discuss openly about it, and always reminded me to take a good care of Yuni. I am really grateful to know and work with you Heleen. I want to express my gratitude to all the colleagues and staffs of PBR who made it easier and safe for all the $\mathrm{PhD}$ students like me to do practical work in the molecular lab, especially Jos, Linda, Marian, Johan, Gert, Marjon, Wendy, Martijn, Annelies, Gerard, Fien, Doret, Danny, Koen, and Irma; and in the greenhouse, especially Dirkjan, and Andre, Gerrit, Taede, and Rohan from Unifarm. To our beloved secretaries Janneke, Nicole and Letty, thank you very much for being supportive to students like me, and for helping us arrange all kinds of things for our courses, conferences, poster printing, etc. You often have time to listen to and share with me things going on with family life, which I appreciate a lot. Thanks again for your support and kindness. Furthermore, I also would like to thank all the rest of colleagues in PBR, whom I had privilege to work with, have interaction with, and share fun time together during work as well as activities outside work, especially Dianka, Christel, late Luc, Robert, Oene, Elma, Remmelt of BBE research group, Arnaud, Yury, Gerard, Christian, Jack, Patrick, Paul, Herman, Alex, Daniel, Anne-Marie, Rene, Yuling, Jan Kees, Marcel, Sjaak, Olga, Henk, and Jan.

I feel also very grateful that I have many accompanied colleagues that have been going through the journey of doing $\mathrm{PhD}$ or post-doc projects, whom I could share all the ups and downs, tears and laughter. First I would like to thank my beloved PhD officemates whom have brightened the atmosphere of the office: Arwa, Mirjana, Johan, Ashikin, Kaile, and Jeroen. Thanks for sharing all kinds of things with me, from the war-torn situation in the home country to the welcoming of your first-born. To Arwa, thank you for being very hospitable, for opening up your house to host me and Yuni with delicious Syrian food and nice evening with games together. I am fortunate enough to be born after the war in Vietnam, thus I have no idea how it's like to when you and your loved ones have to go through it. I'm sure it is not nice. I do hope that the peace will come soon one day to your country. To Jihua, Weicong, Luis, Tim, Andres, Behzad, Claire, Jordi, Jarst, and Michiel, I did enjoy the fun and interaction that we had together under Luisa's group. I can finally join the graduate group with you guys. Izan and Alessandra, thank you for all the fun time that we did together also with Xuan, going for nice lunches between our works, or just lying on the grass in front of Forum enjoying the sun, also the crazy time that we did the ice bucket challenge together 
with Xuan. Claire, thanks for the nice interactions, for your life journey that you shared with me. I'm thankful to see you grow stronger and stronger. I am also very grateful to all the other $\mathrm{PhD}$ and post-doc colleagues at PBR that I have chance to interact with during many activities, i.e. Anne, Michela, Mas, Juan, Freddy, Yusuf, Atiyeh, Thijs, Narges, Suxian, Alejandro, Wei, Dong, Yigian, Nan, Mehdi, Michela, Abe, Valentina, Myluska, Charlie, Marine, Xiao, Cynara, Sara, Christos, Peter B., Viviana, Carolina, Kim, Mathilde, Aurelie, and Pauline. Especially to Rafa-Marce, Paula-Cesar, Madhu-Manoj, thanks for all the nice meals and fun time that we shared. To Mas, you are such a lively colleague that I have privilege to know. You can bring your laughter everywhere, your baking is so good, and you are full of energy in organizing many activities with delicious food and fun games for us. Thank you, you are such a blessing! In addition, I also want to express my gratitude to all the $\mathrm{PhD}$ colleagues that have accompanied me in many meetings and activities within the Towards BioSolar Cell consortium, i.e. Alena, Ievgeniia, Graham, and Luwik. Susan, thank you for your timely help with arranging the education statement.

Because I like sports a lot, while writing this, many memories with sports events flashed back, teams "Plants attack!" and "Naked plants" for zeskamp competition during WE-DAY 2014 and 2016, sports night with team "Dream Catcher" in 2015, I did enjoy every bit of it! Looking at those photos makes me laugh so much. I just to call out and thank those that made those events so memorable: teams "Plant Attacks" with Tim, Behzad, Marcel, Dalia, Valentina, Xuan, and Ernest; team "Dreamcatcher" with Tim, Xuan, Xiao, and Jack; team "Naked Plants" with Tim, Xuan, Johan, Rients, Vivi, Andres, Marieke, Jeroen, and Anne. Besides these events, I also really enjoyed training and playing badminton with De Lobbers club. I especially want to thank my teammates Niels, Stephanie, Sabina, Linda, Nienke, Marjon, Vincent, and Chen for all competition, tournaments, and fun activities that we did together. Also my special thanks to the student community of badminton lovers, who helped me stay active on Fridays'evening and weekends in the sports centre de Bongerds.

To Xuan, my best buddy, I'm so glad that I can thank you now, finally! Thank you for sharing many ups and downs with me during the PhD journey. I admire you for your kindness, cheerfulness, creativeness, and hard-working spirit! You can connect with people so easily, and always give $100 \%$ energy in everything you do, work or play. You were there helping me with plant harvesting and phenotyping, fighting all the battles with teams "Plant Attacks", "Dream-catchers", and "Naked plants", encouraging each other with the writing parts of our $\mathrm{PhD}$ journey as writing buddy, sharing all the nice and relaxing lunches between our works. You were also the very first person together with some other colleagues that listened to and interacted with me during the lunch break of 28 August 2013 while we talked about upcoming Christmas holiday, and I tried to explain the real meaning of Christmas that I had just understood not so long before that. I remember this date so well because it had a big impact on my life. Thank you for being a part of it. And I'm so thankful that you always encourage me to push to the finish line, and also agree to be my paranymph, I do look forward to celebrating this important day with you, Xuan! 
To Ernest, my brother! Thank you for the bonding that you have shared with me since we have met during the period of doing Master study. Thank you for also helping a lot with plant harvesting and phenotyping, fighting the zeskamp battles with team "Plant Attacks", joining the music team at ICF together. You are also full of energy, which really can influence the surrounding in a positive way. I can't keep myself from laughing when I think back the time that you kept rolling on the floor laughing so hard when we were talking of something funny. You have such a surreal calmness when you have encountered many difficult issues with your works, which helped you to keep pushing forward to overcome it. It's really inspiring. I also admire you a lot that you are willing to spend a lot of time reaching out and sharing with people the love and hope that you have inside you, and encourage people when they are in difficult situations. I and my family look forward to seeing you, Florence, and Great more often as "neighbors" soon in Almere! And thank you for being my paranymph, for spending time to help me with the cover and invitation card design! I look forward to celebrating this special day with you Ernest!

Being far away from home, there are a few things that I missed, i.e. speaking Vietnamese and eating Vietnamese food (I don't cook well unfortunately). Thankfully I have met many Vietnamese friends in Wageningen and had a lot of fun. Among those, I want to thank especially for making so many precious memories. Chi Ai, you are so nice and you treat me like your younger brother, for which I feel really blessed. You always cook so many good Vietnamese and other foods for me, Yuni, and Elsa, we always go home with a very happy belly (-) To Jacob, thank you for always together with chi Ai being so hospitable to us, and for being so generous in giving many things to Elsa. Jacob, it was at your place when I joined with Yuni for the first time for Bible study. Later on you got married to chi Ai, and I got married to Yuni. It's just amazing to see how we are connected. And thank Jacob and chi Ai for helping me and Yuni a lot for the day that we signed the paper to commit to our marriage at Wageningen gementee. You both are really like our family! Nam \& Tien, thank you for the friendship, for all the nice time playing badminton and activities that we did together. Chi Minh - anh Duy, thank you for the friendship, all the fun things and trips that we did since we have met in the Netherlands in 2009. It's just amazing that we could keep in touch and that anh Duy now works in the same company Enza Zaden with me. Hopefully we can be neighbor soon in Almere, and we can make more trips with the whole families in the near future! I also want to express my gratitude to chi Huong, chi Dung, anh Long - chi Lan, anh $\mathrm{Vu}$ - chi Minh, anh Hoang, anh Ke - chi Quyen, anh Cuong - chi Van Anh, Phuong, Long, $\mathrm{Ha}$, and many more for making me feel more like home during my stay in Wageningen.

Furthermore, I want express my deepest gratitude to the church family of International Christian Fellowship (ICF) Wageningen. I was so drawn to love that people in ICF shared with each other and to new students in town like me. People opened up their home to welcome each other for meals, offer practical helps, organize fun activities (Dutch courses, city trips, game nights, cultural evenings, etc.), learn more about Bible, pray together, open to any tough questions about any topics and try their best to provide an answer to those. I want to thank especially the following people that have touched my life and helped it turn to better direction: Connie, Johan - Heleen, Koos - Carolien, Daniel - Hilda, Simon, Gerwin - Fillipa, 
Alvaro - Mina, Sebastiaan - Marije, Adnan - Linda, Koenraad - Andrea, Gerson - Maiga, Han - Marijke, Octavina - Andy, Jugk, Tamar \& Nana, Maarten, Andrea, Adriaan, Aaron, Elly, Henk's family, pastor Dick. I want to especially thank Johan for being my mentor, for guiding me in making the important decisions, for showing me an inspiring example of a family life, love and care for people. Thanks Koos, Caroliene, Tom, Thijs, and Cris for always thinking about and being there for my family. Daniel, thank you for your dedication and enthusiasm with organizing so many fun activities, I enjoyed working with you in the activity committee a lot. Before I came to ICF, I was so broken inside and ashamed, I managed to hide it at first but was able to share the brokenness later to people whom I got to know better at ICF. And then I was healed bit by bit from the inside, I found hope and purpose, and feel so alive. Words cannot describe enough how thankful I am to the whole ICF family. I do hope that ICF will keep being the loving community where students like me from every walk of life can feel safe to be vulnerable and to find healing, love, peace, and joy. One of the most memorable activities was the day that ICF team spent with the refugees in AZC of Wageningen. It's heartwarming to see many smiles and laughter while all of us could play games, sports, and eat together. I also want to thank Josine of Wageningen Student Chaplaincy for many memorable activities that you organized with all the students. One of them that I will stay in my heart for very long is the trips that you organized to visit prisoners during Christmas period, to sing, talk and share a meal together. To Alexander of Chaplaincy, Johan of ICF and Maria of International Catholic Community (ICC), thank you for standing up, together finding solutions, and organizing activities to tackle the issue of loneliness/ depression/ suicide in the student building of Bornsesteeg. Maria, thank you for also sharing the challenging journey that you have been going through with the cancer treatment. I really admire your courage and kindness. I hope you will completely recover soon! To Nazri my neighbor at $\mathrm{PhD}$ apartment Lombardi, thank you for the friendship, for the time that we play badminton together, the meals that we share, the respectful conversions that we had about our different faiths. Sorry that I didn't manage to visit last time in Malaysia, hopefully we can meet again somehow somewhere in the near future. I also want to express my gratitude to all the members of the New Day International Church (NDIC) in Almere for welcoming me and my family, for treating us so kind, for providing so much care, especially during the weeks after Yuni had given birth to Elsa. Being new parents with short-night sleeps, having the meals delivered to our house every day by different members of the church family really touched us. We want to thank especially pastors John \& Joy, Benedicta family, Phoebe, Samuel \& Johan, Julian, Ross \& Assie, Kweku \& Esther, Jeoren \& Sow Kheng, Matthew \& Danielle, and Sibbelienk for their love and care.

I also want to take this chance to thank all the colleagues at Enza Biotechnology Research and Application department for the nice working environment that I am privileged to experience now. I would like to first especially thank the colleagues who have worked very closely and supported me to grow more in doing research, in various skills to work better in team, i.e. Richard, Willeke, Bastiaan, Fausto, and Gert-Jan. Thanks for the support and encouragement! I also want to express my gratitude to the following colleagues that I also have had opportunities to work and interact with: Sjaak, Simon, Bas tR, Faira, Eshter, Ilona, Ilja, Bart, Melanie, Walter, Marieke, Nejra, Jan-Willem, Janita, Jurre, Bas vH, Wiebe, 
Annabel, Valerio, Paulien, Annemarie, Lorena, Pallavi, Tieme, Vincent, Lan Anh, Julia, Samy, Benjamin, Albert, Lex, Hugo, Siyang, Robin, Nadia, Saskia, Willy, Mike, Kim, and Mathieu.

Viết được những lời cảm ơn ngày hôm nay trong cuốn sách luận án tiến sĩ này thật sư là điều ngoài sự tưởng tượng của con. Tất cả nhờ đến hồng ân Chúa! Con cảm ơn Chúa đã ban cho con gia đình luôn yêu thương con, mặc dù con đã rất nhiều lần làm gia đình buồn lòng về sự lêu lỏng của con đặc biệt lúc con còn ở VN. Bây giờ có Elsa, con mới biết rõ hơn là tình yêu của ba mẹ đặc biệt như nào. Con đặc biệt muốn cảm ơn đến ba mẹ đã luôn quan tâm, động viên và cầu nguyện cho con và gia đình bên Hà Lan ngày đêm. Đặc biệt là mẹ luôn cố gắng làm lụng để con cái và gia đình được ấm no đầy đủ, và luôn chia sẻ cùng con mọi chuyện, luôn sẵn lòng giúp đỡ mọi người, trong lúc khó khăn nhất mẹ cũng luôn vững niềm tin. Mẹ là tấm gương cho con noi theo. Ba, con kiên nhẫn và quyết tâm làm và viết xong luận án là nhờ hồng ân Chúa và rất nhiều người quan tâm và động viên. Ba cũng kiên nhẫn và quyết tâm trị bệnh nhé, cả nhà sẽ luôn động viên và cầu nguyện cho ba, con tin là ba sẽ khỏe hơn! Dũng cũng cảm ơn Nhung \& Bảo và Lụa và Gấu, Quí \& Oanh và cu Bi đã luôn nhiệt tình đón tiếp Dũng, Yuni và Elsa mỗi dịp được gặp mặt. Quí cố gắng nhé, Chúa thay đổi Dũng được, thì Chúa cũng sẽ thay đổi Quí được nếu Quí tìm chơi với những người bạn tốt biết quan tâm và động viên Quí đúng cách! Bà ngoại và ông bà nội, con hy vọng mọi sẽ hãnh diện khi biết tin này trên thiên đàng. Bác Chính, bác Lạc, bác Thụy, cháu rất cảm ơn các bác đã giúp cháu rất nhiều, tạo điều kiện cho cháu ăn học ở Hà Lan. Không có sự giúp đỡ của các bác, cháu không biết bây giờ cháu sẽ ra sao. Hy vọng các bác cũng sẽ hãnh diện và vui khi biết tin này trên thiên đàng. Cháu cũng cảm ơn bác Hiền, Giang, và Ân đã đón tiếp gia đình cháu mỗi lần về thăm. Hy vọng cả gia đình ở VN sẽ có dịp qua HL chơi với tụi cháu sớm! Cháu cũng cảm ơn bác Khẩn và Gia Linh rất nhiều vì đã cưu mang cháu 4 năm học đầu tiên ở Hà Lan. Đặc biệt là bác Khẩn, tụi cháu biết ơn bác nhiều lắm, bác luôn nấu ăn ngon cho tụi cháu và còn cho rất nhiều đồ mang về, đặc biệt là món bánh cuốn rất ngon của bác $:$ : Cháu cũng xin cảm ơn gia đình cậu Bình \& mợ Ly, cậu Sơn \& mợ Hương đã quan tâm tới cháu và gia đình đặc biệt lúc cháu còn bỡ ngỡ ở Hà Lan. Cảm chị Liên Hương \& Loek và Lara cũng đã quan tâm, động viên em, và luôn đãi gia đình em ăn ngon mỗi lần đến thăm.

I also want to express my deepest gratitude to my family in law who has played such a big role in changing my life for the better. I only met you papa, mama, and Ahau for a few days during the trip that you made to NL to celebrate Yuni's MSc graduation ceremony in Wageningen in 2010. After that there was no contact at all between us, and suddenly at the beginning of my PhD project in December 2011, I asked your permission to get married to Yuni and you agreed. This is not usual at all in our cultures because the family usually needs to know the man wanting to ask the hands of their daughter well before giving him their blessing. Especially papa, mama, terima kasih banyak! Your blessing to let me building a family with Yuni had created an exciting chapter in my life that I never could dream off. Thank you papa, mama, Ahau, Asiong for trusting me! Thank you for all the love that you have for us, for all the nice trips that you arranged for us. Hopefully we can meet many more times and make more memorable trips in the future $:-$ I also want to express my appreciation 
to I-Siang, Soi-Cek, Soi-Sing, Willy \& Melvin for their love and care every time we have had chance to meet.

Finally, to my beloved wife Yuni and daughter Elsa, words are not enough to describe how blessed I am with you in my life. Sayang, thank you for loving me, helping me to change for the better, accompanying me through this $\mathrm{PhD}$ journey, and building up family together. You were the very first one that I shared my struggle of pornography addiction to, and you didn't give up on me despite my constant failures to keep my promise to overcome it. You brought me to the loving family ICF, through which I could find healing, joy, and hope. Through the enduring support from you and ICF family, I was finally free of this addiction and was so grateful for it. It is so great to feel this kind of freedom. Thanks to this freedom, I could have my mind freer to enjoy real life, works, and interaction with people. It's a good news to know that there is always hope when you have people around who keep loving and supporting you. Thank you for being patient with me, and being there with me through all the ups and downs. I'm thankful for your sacrifice so that I could have more time to complete the thesis writing. I will strive to keep growing more maturely as time goes by, so that I can accompany you to continue strengthening our love and our family. Elsa, my little sunshine, when you grow older and can read these words, papa want you to know that papa love you a lot! Papa is really thankful to have you in papa's life! You give me such a humble experience to be a dad for the first time, which help papa now can understand a bit more of what the father's love is like. Papa hope that together with mama we will be able to keep learning to be good parents and loving you and your expecting baby sister in many more years to come, and that you both will be surrounded with people who love and support you to experience life and be a blessing for people around you.

Once again, I'm deeply grateful to all of you who have been supporting, guiding, and loving me through all these 9 long years, so that I can finally reach this milestone in my life. I hope we can keep in touch as long as possible. I do treasure these memories, lessons, experiences that I have learnt in this period. As life goes on, I will strive to keep growing and learning, so that I can contribute in different ways as long as I have privilege to live, work, and serve.

\section{Quy Dung - Peter}

Almere, August 2020 


\section{About the author}

Quy Dung (Peter) Dinh was born on $16^{\text {th }}$ May 1984 in Ho Chi Minh city, Vietnam. In 2004, he moved to Nijmegen in the Netherlands to study and obtained Bachelor degree in Life Sciences program at Hogeschool van Arnhem en Nijmegen. After that, he worked as a research assistant at Plant Research International in Wageningen (2008 - 2009). Subsequently Quy Dung studied and obtained his Master degree in Plant Sciences at Wageningen University (2009 - 2011). In October 2011 he started his PhD research at Plant Breeding department of Wageningen University and Research under supervision of Prof. Dr. Luisa Trindade and Prof. Dr. Richard Visser. In this thesis, the results obtained during his PhD period are presented. From July 2016 he has been working at Molecular Biology and Biochemistry department of Enza Zaden Research and Development B.V. in Enkhuizen. He currently lives in Almere with his wife Yuni and daughter Elsa.

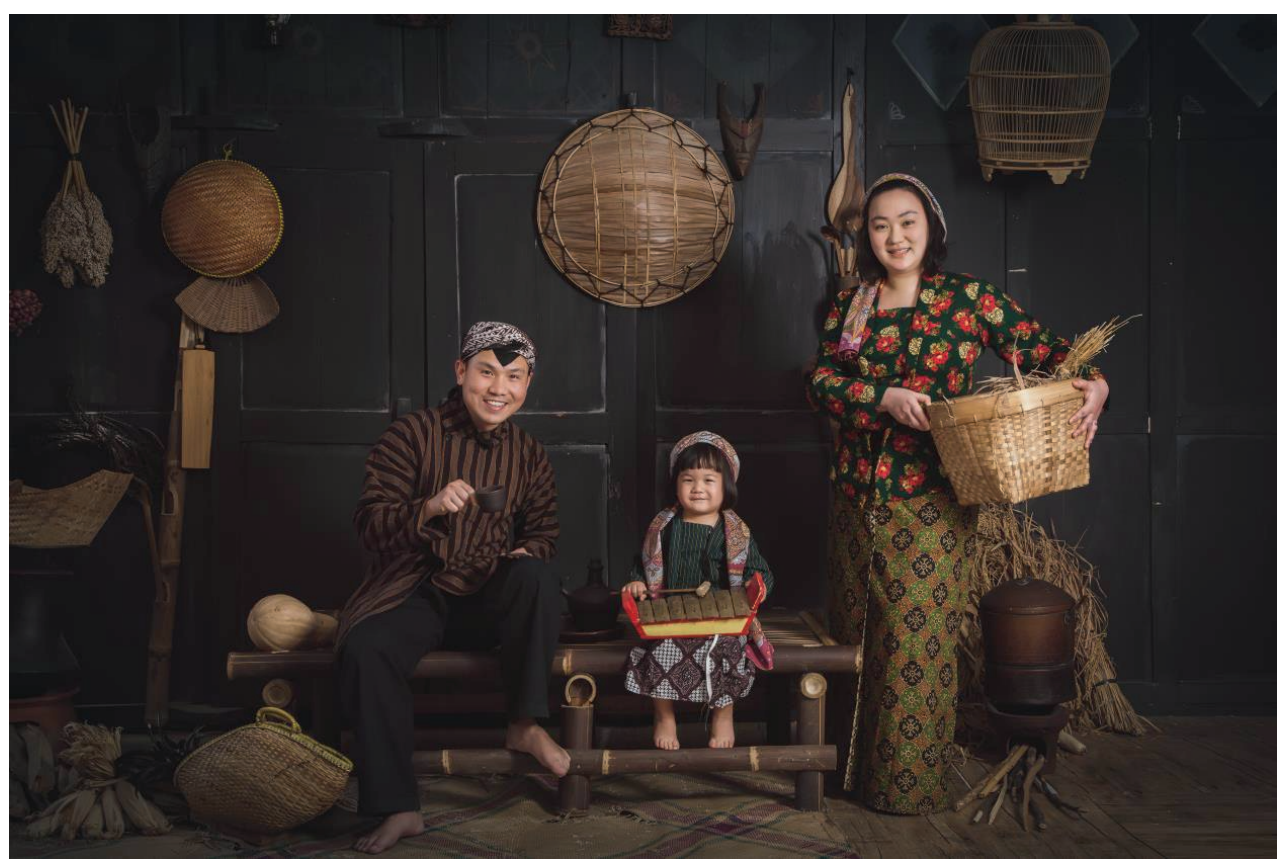




\section{List of publications}

Dinh, Q.-D., A. Dechesne, H. Furrer, G. Taylor, R. G. F. Visser, J. Harbinson and L. M. Trindade (2019). "High-Altitude Wild Species Solanum arcanum LA385-A Potential Source for Improvement of Plant Growth and Photosynthetic Performance at Suboptimal Temperatures." Frontiers in Plant Science 10(1163).

Dinh, Q.-D., R. Finkers, A. H. Westphal, W. M. A. M. van Dongen, R. G. F. Visser and L. M. Trindade (2018). "Exploring natural genetic variation in tomato sucrose synthases on the basis of increased kinetic properties." PLOS ONE 13(10): e0206636.

van Noorden, G. E., R. Verbeek, Q. D. Dinh, J. Jin, A. Green, J. L. P. Ng and U. Mathesius (2016). "Molecular Signals Controlling the Inhibition of Nodulation by Nitrate in Medicago truncatula." International journal of molecular sciences 17(7): 1060.

Smaczniak, C., R. G. H. Immink, J. M. Muiño, R. Blanvillain, M. Busscher, J. BusscherLange, Q. D. (Peter) Dinh, S. Liu, A. H. Westphal, S. Boeren, F. Parcy, L. Xu, C. C. Carles, G. C. Angenent and K. Kaufmann (2012). "Characterization of MADSdomain transcription factor complexes in Arabidopsis flower development." Proceedings of the National Academy of Sciences 109(5): 1560-1565.

Urbanus, S. L., Q. D. (Peter) Dinh, G. C. Angenent and R. G. H. Immink (2010). "Investigation of MADS domain transcription factor dynamics in the floral meristem." Plant signaling \& behavior 5(10): 1260-1262.

Urbanus, S. L., A. P. Martinelli, Q. D. (Peter) Dinh, L. C. Aizza, M. C. Dornelas, G. C. Angenent and R. G. Immink (2010). "Intercellular transport of epidermis-expressed MADS domain transcription factors and their effect on plant morphology and floral transition." The Plant Journal 63(1): 60-72. 


\title{
Education statement of the Graduate School Experimental Plant Sciences
}

\author{
Issued to : Quy Dung Dinh \\ Date : :9 September 2020 \\ Group : Plant Breeding \\ University : Wageningen University \& Research
}

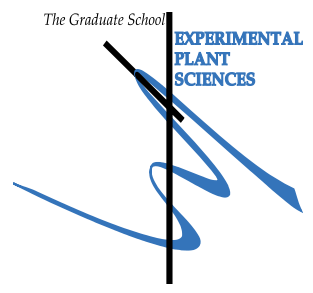

\section{1) Start-Up Phase}

- First presentation of your project Preventing feedback inhibition of photosynthesis

- Writing or rewriting a project proposal Photosynthesis feedback inhibition

- Writing a review or book chapter

- MSc courses

\section{date}

Feb 14, 2012

Oct 2011 - Feb 2012

Subtotal Start-Up Phase

\section{2) Scientific Exposure}

\section{date}

\section{- EPS PhD student days}

EPS PhD student day, Amsterdam

Nov 30, 2012

EPS PhD student day, Leiden

Nov 29, 2013

- EPS theme symposia

EPS theme 4 Genome Biology symposium 2011, Wageningen

Dec 09, 2011

EPS theme 3 Metabolism \& Adaptation symposium 2013,

Amsterdam

Mar 22, 2013

EPS theme 3 Metabolism \& Adaptation symposium 2014, Wageningen

Mar 11, 2014

- Lunteren Days and other national platforms

Annual Meeting Experimental Plant Sciences 2012, Lunteren

Apr 02-03, 2012

Annual Meeting Experimental Plant Sciences 2013, Lunteren

Apr 22-23, 2013

BioSolar Cells Consortium annual meeting, Wageningen

May 10-11, 2012

BioSolar Cells Consortium annual meeting, Ede

Jun 13-14, 2013

BioSolar Cells Consortium autumn meeting, Amsterdam

Nov 28, 2013

BioSolar Cells Consortium half year meeting, Wageningen

Dec 12, 2014 


\section{- Seminars (series), workshops and symposia}

Mini-symposium 'Plant Breeding in the Genomics Era',

Wageningen

Nov 25, 2011

EPS Flying seminar Cornelia Spetea Wiklund, Wageningen

Feb 21, 2012

Plant Breeding Research Day 2012, Wageningen

Feb 28, 2012

Mini-symposium Plant Molecular Physiology, Utrecht

Nov 20, 2012

BioSolar Cells Valorisation Day, Utrecht

Mar 15, 2013

Workshop 'Applications of X-ray computed tomography (XRT)

and live demonstration', Wageningen

June 18, 2013

B-wise: Bioinformatics Seminar, Wageningen

May 6, 2014

Plant Breeding Research Day 2014, Wageningen

Sept 30, 2014

Plant Breeding Research Day 2015, Wageningen

Sept 29, 2015

\section{- Seminar plus}

\section{- International symposia and congresses}

Next Generation Plant Breeding conference (theme 4 session),

Nov 14, 2012

Ede

European Plant Science PhD Retreat 2014, Amsterdam

July 1-4, 2014

BioSolar Cells Consortium annual meeting with international

Jun 23-24, 2014 programme, Wageningen

BioSolar Cells Consortium annual meeting with international

Jun 17-18, 2015 programme, Ede

\section{- Presentations}

Oral presentation BioSolar Cells Consortium kick-off meeting,

Oct 25, 2011

Wageningen

Poster Presentation at BioSolar Cells Consortium annual

May 10-11, 2012 meeting, Wageningen

Oral presentation at EPS PhD student day 2012, Amsterdam

Nov 23, 2012

Poster and oral presentation presentation at BioSolar Cells

Jun 13-14, 2013

annual meeting, Wageningen

Oral presentation at European Plant Science PhD Retreat 2014,

Jul 1-4, 2014

Amsterdam

Poster and oral presentation at BioSolar Cells Consortium

Jun 23-24, 2014

annual meeting, Ede

Oral presentation at BioSolar Cells Consortium half year

Dec 12, 2014 meeting, Wageningen

Poster and oral presentation at BioSolar Cells Consortium

Jun 17-18, 2015 annual meeting, Ede

\section{- IAB interview}

- Excursions

Company visit Genetwister and In2care, Wageningen

Sep 19, 2014 


\section{3) In-Depth Studies}

date

- Advanced scientific courses \& workshops

$\mathrm{PhD}$ course 'Bioinformatics - a User's Approach', Wageningen

Aug 27-31, 2012

3rd transPLANT user training workshop 'Exploiting and

Oct 13-14, 2014

understanding Solanaceous genomes', Wageningen

- Journal club

Literature discussion, Plant Breeding, Wageningen

Oct 2011 - Oct 2015

Individual research training

Subtotal In-Depth Studies

\section{4) Personal Development}

\section{- General skill training courses}

Seminar 'Does gender matter (in management)?', Wageningen

Competence Assessment, Wageningen

Course 'Project and Time Management', Wageningen

Mini-symposium 'How to Write a World-class Paper',

Wageningen

PhD Workshop Carousel, Wageningen

Course 'Scientific Writing', Wageningen

Course 'Career Orientation', Wageningen date

Nov 8, 2011

Mar - Apr, 2012

Apr - Jun 2012

Oct 17, 2013

Jun 02, 2014

Sep - Nov, 2015

Nov - Dec, 2015

Apr 2012 - May 2013

Organising Monday Seminars at Plant Breeding WUR

\section{2

\section{TOTAL NUMBER OF CREDIT POINTS*}

32.8

Herewith the Graduate School declares that the PhD candidate has complied with the educational requirements set by the Educational Committee of EPS with a minimum total of 30 ECTS credits.

* A credit represents a normative study load of 28 hours of study. 
The work described in this thesis was conducted within the research programme Towards BioSolar Cells and co-financed by the Dutch Ministry of Economic Affairs.

The printing of this thesis is financially supported by Plant Breeding - Wageningen University \& Research.

Cover and background photos by Roman Penderev and Hywit Dimyadi / Shutterstock.com Layout by Quy Dung Dinh, Cover design by Ernest Aliche Printed by Digiforce | ProefschriftMaken 



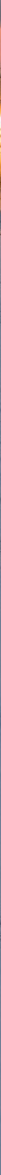

